\title{
HYPERSONIC EXPERIMENTAL AERO-THERMAL CAPABILITY STUDY THROUGH MULTILEVEL FIDELITY COMPUTATIONAL FLUID DYNAMICS
}

\author{
Thesis \\ Submitted to \\ The School of Engineering of the \\ UNIVERSITY OF DAYTON \\ In Partial Fulfillment of the Requirements for \\ The Degree of \\ Master of Science in Aerospace Engineering \\ By \\ Denton Gregory Sagerman \\ UNIVERSITY OF DAYTON \\ Dayton, Ohio \\ August, 2017
}




\section{HYPERSONIC EXPERIMENTAL AERO-THERMAL CAPABILITY STUDY THROUGH MULTILEVEL FIDELITY COMPUTATIONAL FLUID DYNAMICS}

Name: Sagerman, Denton Gregory

\section{APPROVED BY:}

Markus P. Rumpfkeil, Ph.D.

Advisor Committee Chairman

Associate Professor, Mechanical and

Aerospace Engineering
Aaron Altman, Ph.D.

Committee Member

Professor, Mechanical and Aerospace

Engineering

José Camberos, Ph.D., P.E.

Committee Member

Aerospace Engineer, AFRL/RQHV

Robert J. Wilkens, Ph.D., P.E.

Associate Dean for Research and

Innovation

Professor, School of Engineering
Eddy M. Rojas, Ph.D., M.A., P. E.

Dean, School of Engineering 


\section{(C) Copyright by}

\section{Denton Gregory Sagerman}

All rights reserved 


\begin{abstract}
HYPERSONIC EXPERIMENTAL AERO-THERMAL CAPABILITY STUDY THROUGH

MULTILEVEL FIDELITY COMPUTATIONAL FLUID DYNAMICS
\end{abstract}

Name: Sagerman, Denton Gregory

University of Dayton

Advisor: Dr. Markus P. Rumpfkeil

As true with all hypersonic flight, the ability to quickly and accurately predict the aero-thermodynamic response of an aircraft in the early design phase is important to not only lower cost, but also to lower the computational and experimental time required to test various parameters. The Mach 6 High Reynolds Number Facility at Wright-Patterson Air Force Base in Dayton, Ohio has been nonoperational for the past twenty years, but a recent resurgence in the need for accurate hypersonic test facilities has led to the reactivation of the wind tunnel. With its restoration, new capabilities to assess hypersonic aero-thermodynamic effects on bodies in Mach 6 flow have emerged. Therefore, the objective of this research is to determine if obtaining aero-thermal data from the Mach 6 tunnel using temperature sensitive paint (TSP) is a viable option. Surface pressure and temperature readings, from pressure taps and thermocouples installed on the models, as well as TSP wall temperature distributions will be used for comparison with results from computational fluid dynamics (CFD) analysis codes of differing fidelity levels. The comparisons can then be utilized to gain confidence in the ability of the tunnel to capture the aero-thermal response of complex geometries. Three computational codes were used for numerical comparisons: the Configuration Based 
Aerodynamics (CBAero) tool set, an inviscid panel code with viscous approximation capabilities, Cart3d coupled with Unstructured Langley Approximate Three-Dimensional Convective Heating (UNLATCH) code to approximate viscous effects from the Euler solution, and finally Fun3d, a fully viscous RANS solver. The three tunnel model geometries that will be used for this research are the Reference Flight System model G (RFSG), a Generic Hypersonic Vehicle (GHV), and the Hypersonic International Flight Research Experimentation Program-Flight 1 (HIFiRE-1) payload geometry. 
I would like to dedicate this work to my parents Greg and Karen who have been instrumental in my pursuit in the field of academics. Without their constant support I would not be the man I am today.

I would like to also dedicate this thesis to all my friends, family and all of those who laid the groundwork before me as without them the work I was able to do would not be possible. 


\section{ACKNOWLEDGMENTS}

I would like to thank Barry Hellman for sponsoring this project and giving me the opportunity to learn and grow as an engineer. The tools and concepts I have been exposed to during this project have given me a great set of skills which I will be able to use in my future career. I would not be in this position if it were not for my undergraduate adviser Dr. Aaron Altman. His constant obsession with learning and teaching have inspired me to never be content with the easy answer and to always value the process more than the final result. Dr. Altman is a mentor in every sense of the word and I thank him for the profound impact he made and continues to make on me and many more students like me. I would also like to thank Dave Adamczak, James Tancred, Rebecca Hoffman and Nastasja Dasque for their help mentoring me throughout the process and being willing to answer any questions I had. Without them this project would not have been be a success. Finally, I would like to thank my graduate adviser Dr. Markus Rumpfkeil for his wealth of insight and dedication to my personal learning throughout this project and my academic journey. Whether it be having you as a professor, being your teaching assistant or having you as an adviser, I am truly going to miss working with you as you have been extremely influential in my career. 


\section{TABLE OF CONTENTS}

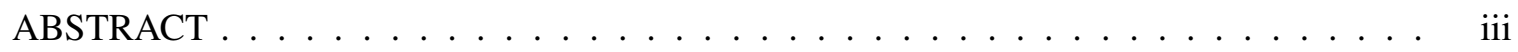

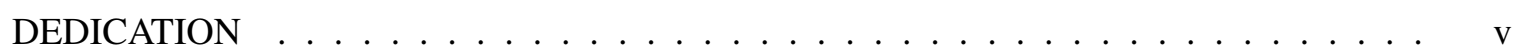

ACKNOWLEDGMENTS . . . . . . . . . . . . . . . . . . . . . . . vi vi

LIST OF FIGURES . . . . . . . . . . . . . . . . . . . . . . . . . . ix

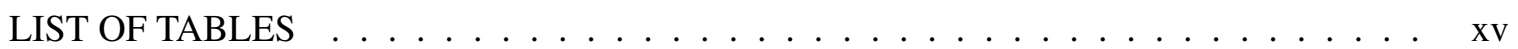

LIST OF SYMBOLS $\ldots \ldots \ldots \ldots \ldots \ldots \ldots \ldots \ldots \ldots \ldots \ldots$ xvi

CHAPTER I. INTRODUCTION AND BACKGROUND $\ldots \ldots \ldots \ldots \ldots \ldots$

1.1 Overview of Hypersonic Flow . . . . . . . . . . . . . . 1

1.2 Brief History of Hypersonics $\ldots \ldots \ldots \ldots \ldots$

1.2 .1 The Birth of Hypersonics . . . . . . . . . . . . . . 4

1.2 .2 Major Breakthrough: The X-15 . . . . . . . . . . . . 4

1.2 .3 Propelling the Future: The X-51 Waverider . . . . . . . 5

1.3 Hypersonic Experiments . . . . . . . . . . . . . . . . 6

1.4 Hypersonic Numerical Simulations . . . . . . . . . . . . . . . . 9

1.5 Objectives and Accomplishments of this Research . . . . . . . . . 9

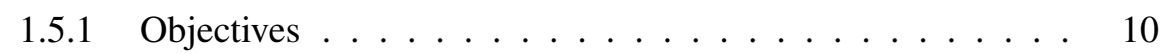

1.5 .2 Accomplishments . . . . . . . . . . . . . 11

CHAPTER II. MACH 6 WIND TUNNEL EXPERIMENTAL RESULTS $\ldots \ldots \ldots \ldots$.

2.1 The Mach 6 Tunnel . . . . . . . . . . . . . . . . . . . 13

$2.1 .1 \quad$ Initial Paint Adhesion Testing . . . . . . . . . . . . . 15

2.2 Geometries . . . . . . . . . . . . . . . . . . . 17

2.3 Experimental Testing $\ldots \ldots \ldots \ldots \ldots \ldots \ldots \ldots$

$2.3 .1 \quad$ HIFiRE-1 Results $\ldots \ldots \ldots \ldots \ldots \ldots$

$2.3 .2 \quad$ RFSG Results . . . . . . . . . . . . . . . . . . . . . . . . . . 34

2.3 .3 GHV Results . . . . . . . . . . . . . . . . . 41 
CHAPTER III. INVISCID NUMERICAL ANALYSIS $\ldots \ldots \ldots \ldots \ldots$

3.1 CBAero Panel Code Analysis . . . . . . . . . . . . . . . . . . . . . . . . . . . . . 45

3.1 .1 HIFiRE-1 . . . . . . . . . . . . . . . . 47

3.1 .2 RFSG $\ldots \ldots \ldots \ldots \ldots \ldots$

3.1 .3 GHV . . . . . . . . . . . . . . . . . 54

$3.1 .4 \quad$ Limitations in CBAero Analysis $\ldots \ldots \ldots$. . . . . . 57

3.2 CART3D Euler Analysis . . . . . . . . . . . . . . . . . . . . . . . . . . . . . 58

$3.2 .1 \quad$ HIFiRE-1 . . . . . . . . . . . . . . . . . . . . 59

3.2 .2 RFSG $\ldots \ldots \ldots \ldots \ldots \ldots \ldots$

3.2 .3 GHV . . . . . . . . . . . . . . 73

CHAPTER IV. VISCOUS NUMERICAL ANALYSIS $\ldots \ldots \ldots \ldots$. . . . . . . . . . . 82

$4.1 \quad$ UNLATCH Boundary Layer Solver . . . . . . . . . . . . . . . . . 82

$4.1 .1 \quad$ Meshing Strategy for UNLATCH $\ldots \ldots \ldots$. . . . . . . . . . . . . . . . 83

$4.1 .2 \quad$ UNLATCH Results for HIFiRE-1. . . . . . . . . . . . . . . . . . . . . . . . . . . . 85

4.2 FUN3D RANS Solver . . . . . . . . . . . . . . . . . . . . . . . . 87

$4.2 .1 \quad$ Mesh Generation $\ldots \ldots \ldots \ldots$. . . . . . . . . . . . . . . . . . . . . . . . . .

$4.2 .2 \quad$ Input Parameters . . . . . . . . . . . . . . . . . . . 92

4.2 .3 HIFiRE-1 Results . . . . . . . . . . . . . . . . 93

CHAPTER V. $\quad$ EXPERIMENTAL AND NUMERICAL COMPARISON $\ldots \ldots \ldots$

CHAPTER VI. CONCLUSION AND RECOMMENDATIONS. . . . . . . . . . . . . . . 102

6.1 Brief Overview of Project Accomplishments . . . . . . . . . . . 103

6.2 Recommendations and Future Work . . . . . . . . . . . . . . . . 103

BIBLIOGRAPHY . . . . . . . . . . . . . . . . . . . . . 107

APPENDICES

A. HIFiRE-1 Experimental Results . . . . . . . . . . . . . . . . . . . 109

B. Numeca Hexpress/Hybrid Input Script . . . . . . . . . . . . . . . . . . 116

C. Fun3d Input Script for HIFiRE-1 Fine Mesh . . . . . . . . . . . . . . . . . . . 118 


\section{LIST OF FIGURES}

1.1 Hypersonic Program Timeline [1] . . . . . . . . . . . . . . . . . . . . 3

1.2 The X-15 Hypersonic Research Aircraft [2]. . . . . . . . . . . . . . . . . . . . . . 5

$1.3 \quad$ NASA Langley LAL Mach 10 tunnel TSP results [3]. . . . . . . . . . . . . . . 7

$1.4 \quad$ ORNL TSP test model in TGF tunnel [4]. $\ldots \ldots \ldots \ldots$

$1.5 \quad$ TSP results for ORNL model configurations at $M=3$ and $R e=4 \cdot 10^{6}$ [4].]. . . . 8

$2.1 \quad$ Mach 6 High Reynolds Number Wind Tunnel at Wright-Patterson Air Force Base [5]. 14

2.2 Mach 6 Wind Tunnel Layout $[5] . \ldots \ldots \ldots \ldots$

2.3 Paint Adhesion testing models $\ldots \ldots \ldots \ldots \ldots$

2.4 Schlieren images from roughness cone models testing at $\alpha=0$ in Mach 6 tunnel . 17

2.5 Rendered geometries to be tested in Mach 6 High Reynolds Number Wind Tunnel [6]. 18

$2.6 \quad$ HIFiRE-1 rendered CAD model with stingmount. . . . . . . . . . . . . . . . . . 19

$2.7 \quad$ HIFiRE-1 instrumentation layout. . . . . . . . . . . . . . . . . . 20

$2.8 \quad$ HIFiRE-1 unpainted model mounted in Mach 6 Wind Tunnel. . . . . . . . . . 20

$2.9 \quad$ RFSG wind tunnel model with instrumentation . . . . . . . . . . . . . . 22

2.10 Unpainted RFSG model mounted in Mach 6 Wind Tunnel. . . . . . . . . . . . . . 22

2.11 RFSG two-piece model sections. . . . . . . . . . . . . . . . 23 
2.12 GHV wind tunnel model with instrumentation . . . . . . . . . . . . . 25

2.13 Unpainted GHV wind tunnel model. . . . . . . . . . . . . . . . . . . . . . 25

2.14 GHV rendered CAD model in three pieces. . . . . . . . . . . . . . 26

2.15 GHV rendered CAD model side view with sting. . . . . . . . . . . . . . . 27

2.16 TSP calibration curve $\ldots \ldots \ldots \ldots \ldots$

2.17 HIFiRE-1 mounted wind tunnel model. . . . . . . . . . . . . . . . . . . . . 29

2.18 HIFiRE-1 Mach 6 Wind Tunnel results for $C_{p}$ at various AOA for $P_{0}=700 \mathrm{psi}$ and

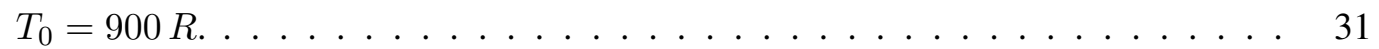

2.19 HIFiRE-1 Mach 6 Wind Tunnel results for surface temperature at various AOA for

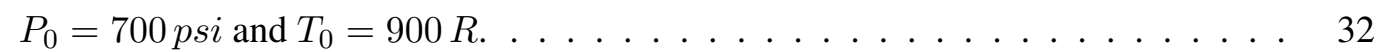

2.20 HIFiRE-1 TSP temperature contours for $P_{0}=700 \mathrm{psi}$ and $T_{0}=900 R$. . . . . 33

2.21 RFSG mounted wind tunnel model $\ldots \ldots \ldots \ldots \ldots$

2.22 RFSG Mach 6 Wind Tunnel results for $C_{p}$ at various AOA for $P_{0}=700$ psi and

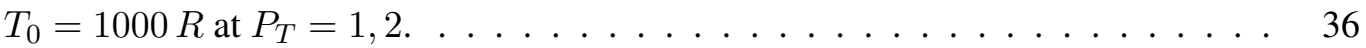

2.23 RFSG Mach 6 Wind Tunnel results for $C_{p}$ at various AOA for $P_{0}=700 \mathrm{psi}$ and

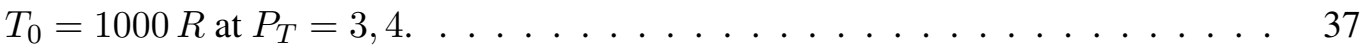

2.24 RFSG Mach 6 Wind Tunnel results for $C_{p}$ at various AOA for $P_{0}=700 \mathrm{psi}$ and

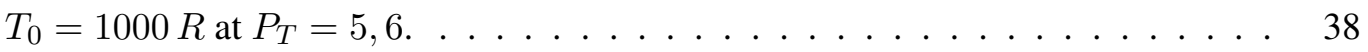

2.25 RFSG Mach 6 Wind Tunnel results for $C_{p}$ at various AOA for $P_{0}=700$ psi and

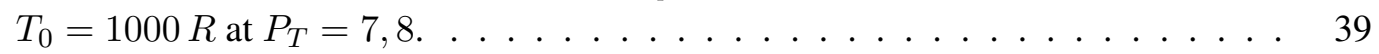

2.26 RFSG Mach 6 Wind Tunnel results for surface temperature at various AOA for $P_{0}=700 p s i$ and $T_{0}=1000 R$ at $T_{c}=1,2,3 . \ldots \ldots \ldots$

2.27 RFSG TSP Temperature Contour. . . . . . . . . . . . . . . . . . . . 41

2.28 GHV mounted wind tunnel model. . . . . . . . . . . . . . . . . . . . 43

2.29 GHV TSP temperature contour for $P_{0}=700 \mathrm{psi}$ and $T_{0}=1000 R$. . . . . . . 44 
$3.1 \quad$ CBAero surface meshes for HIFiRE-1 geometry. $\ldots \ldots \ldots \ldots$. . . . . . . 48

3.2 CBAero Grid Convergence Study for HIFiRE-1 geometry with Notch at 90 deg for various surface meshes $\ldots \ldots \ldots \ldots \ldots$. . . . . . . . . . . . . 49

$3.3 \quad$ Surface heating rates from CBAero for Notch at $90 \mathrm{deg}[6]$. . . . . . . . . . . . . 50

3.4 CBAero surface meshes for the RFSG geometry [6] . . . . . . . . . . . . . . 51

$3.5 \quad$ CBAero Grid Convergence Study for RFSG geometry for various surface meshes . $\quad 52$

$3.6 \quad C_{p}$ distribution from CBAero for RFSG [6] $\ldots \ldots \ldots \ldots \ldots$

$3.7 \quad$ Surface heating rates from CBAero for RFSG [6] $\ldots \ldots \ldots \ldots$

$3.8 \quad$ CBAero surface meshes for the GHV geometry [6] $\ldots \ldots \ldots \ldots$

3.9 CBAero Grid Convergence Study for GHV geometry for various surface meshes. . 56

$3.10 C_{p}$ distribution from CBAero for GHV [6] . . . . . . . . . . . . 57

3.11 HIFiRE-1 Surface Mesh used for CART3D. . . . . . . . . . . . . . . . . . . . 60

3.12 CART3D Volume Meshes through $y$-cut plane. . . . . . . . . . . . . . 60

3.13 CART3D Volume Meshes through $x$ and $y$-cut plane. . . . . . . . . . . . 61

3.14 CART3D Grid Convergence Study for HIFiRE 1 geometry for $C_{L}$. . . . . . . . . 62

3.15 CART3D Grid Convergence Study for HIFiRE 1 geometry for $C_{D} . \ldots$. . . . . . 63

3.16 CART3D Grid Convergence Study for HIFiRE 1 geometry for $C_{M}$. . . . . . . . . 64

3.17 RFSG Surface Mesh with $N_{\text {cells }}=461,492 . \quad \ldots \ldots \ldots$. . . . . . . . . 65

3.18 RFSG Volume Meshes through $y$-cut plane. . . . . . . . . . . . . . . . . 66

3.19 RFSG Volume Meshes through $x$ and $y$-cut plane [6] . . . . . . . . . . . . . 66

3.20 RFSG convergence histories after 9 adaptations. . . . . . . . . . . . . . . 67

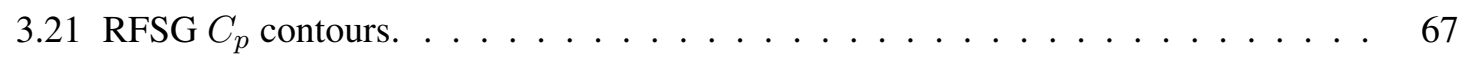




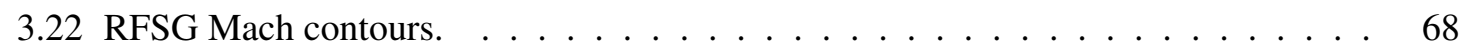

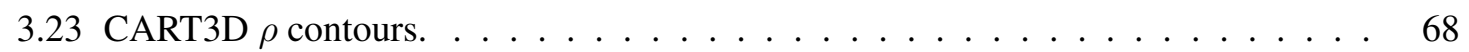

3.24 CART3D Grid Convergence Study for RFSG geometry for $C_{L} . \ldots \ldots$

3.25 CART3D Grid Convergence Study for RFSG geometry for $C_{D}$. . . . . . 70

3.26 CART3D Grid Convergence Study for RFSG geometry for $C_{M}$. . . . . . . . 71

3.27 CART3D vs. CBAero for RFSG along y-cut plane also shown. . . . . . . . . 72

3.28 GHV Surface Mesh with $N_{\text {cells }}=209,196 . \ldots \ldots \ldots \ldots \ldots$

3.29 GHV Volume Meshes through $y$-cut plane. . . . . . . . . . . . . . . . . 74

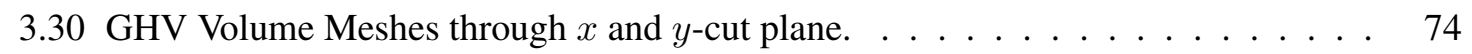

3.31 GHV convergence histories after 8 adaptations. . . . . . . . . . . . . 75

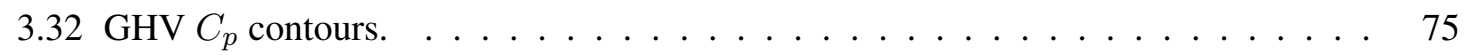

3.33 GHV Mach contours. . . . . . . . . . . . . . . . . . . 76

3.34 GHV $\rho$ contours. . . . . . . . . . . . . . . . . . . 76

3.35 CART3D Grid Convergence Study for GHV geometry for $C_{L}$. . . . . . . . 77

3.36 CART3D Grid Convergence Study for GHV geometry for $C_{D}$. . . . . . . . 78

3.37 CART3D Grid Convergence Study for GHV geometry for $C_{M} . \ldots \ldots$. . . . 79

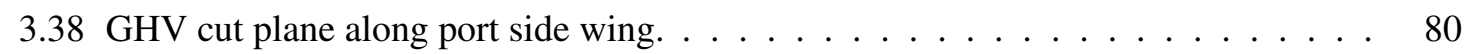

3.39 CART3D vs. CBAero for GHV along $\mathrm{y}$-cut plane of wing. . . . . . . . . . 81

4.1 HIFiRE-1 volume mesh for Cart3d case vs. UNLATCH case at $A O A=0$ degrees. $\quad 84$

4.2 CART3D plus UNLATCH $C_{p}$ contours for HIFiRE-1 geometry. $\ldots \ldots \ldots$

4.3 CART3D plus UNLATCH Mach contours for HIFiRE-1 geometry. . . . . . . . . 85 
$4.4 \quad$ CART3D $\rho$ contours for HIFiRE-1 geometry [6] $\ldots \ldots \ldots \ldots \ldots$

4.5 UNLATCH solution for surface heating rates $\left[\frac{W}{m^{2}}\right]$ for HIFiRE-1 Geometry. . . . . 87

$4.6 \quad$ HIFiRE-1 volume grid for FUN3D, Fine Mesh, $N_{\text {cells }}=21,815,736$. . . . . . . . 91

$4.7 \quad$ HIFiRE-1 volume grid for FUN3D around nose. . . . . . . . . . . . . . . . . . . 92

$4.8 \quad$ HIFiRE-1 FUN3D Convergence History for fine mesh. . . . . . . . . . . . . . . . 94

$4.9 \quad$ HIFiRE-1 FUN3D Grid Convergence Study for $C_{L}$ and $C_{D}$. . . . . . . . . . . 95

4.10 HIFiRE-1 $C_{p}$ contours. . . . . . . . . . . . . . . . . 95

4.11 HIFiRE-1 Mach contours. . . . . . . . . . . . . . . . . . . 96

4.12 HIFiRE $-1 \rho$ contours. . . . . . . . . . . . . . . . . . 96

4.13 HIFiRE-1 $y-p l u s$ contours. . . . . . . . . . . . . . . . 97

4.14 HIFiRE-1 surface heating rates $\left[\frac{W}{m^{2}}\right] \ldots \ldots \ldots \ldots$. . . . . . . . . . 97

5.1 Comparison between experimental and computational $C_{p}$ results for HIFiRE-1 ge-

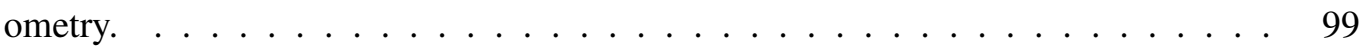

5.2 Comparison between experimental and computational $C_{p}$ results for RFSG geometry. 101

A.1 Mach 6 Wind Tunnel $C_{p}$ results for $P_{0}=700 \mathrm{psi} T_{0}=1000 \mathrm{R}$ at $A O A=-10,-$ $5,0,5,10$ deg with Notch starboard. $\ldots \ldots \ldots \ldots 110$

A.2 Mach 6 Wind Tunnel temperature results for $P_{0}=700 \mathrm{psi} T_{0}=1000 \mathrm{R}$ at $A O A=$ $-10,-5,0,5,10 \mathrm{deg}$ with Notch starboard. . . . . . . . . . . . . . . 111

A.3 Mach 6 Wind Tunnel $C_{p}$ results for $P_{0}=2000 \mathrm{psi} T_{0}=900 \mathrm{R}$ at $A O A=-10,-$ $5,0,5,10$ deg with Notch starboard. . . . . . . . . . . . . . . . 112

A.4 Mach 6 Wind Tunnel temperature results for $P_{0}=2000 \mathrm{psi} T_{0}=900 \mathrm{R}$ at $A O A=$ $-10,-5,0,5,10$ deg with Notch starboard. . . . . . . . . . . . . . . . 113

A.5 Mach 6 Wind Tunnel $C_{p}$ results for $P_{0}=2000 \mathrm{psi} T_{0}=1000 \mathrm{R}$ at $A O A=-10,-$

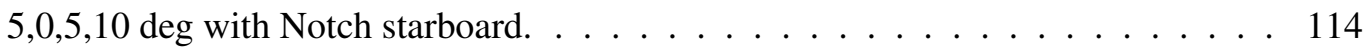


A.6 Mach 6 Wind Tunnel temperature results for $P_{0}=2000 \mathrm{psi} T_{0}=1000 \mathrm{R}$ at $A O A=$ $-10,-5,0,5,10 \mathrm{deg}$ with Notch starboard. . . . . . . . . . . . . . . 115 


\section{LIST OF TABLES}

$2.1 \quad$ Mach 6 Wind Tunnel Testing Conditions [5]. $\ldots \ldots \ldots \ldots$

2.2 Mach 6 Wind Tunnel Testing Matrix for HIFiRE-1 Geometry. . . . . . . . . . . . 29

2.3 Mach 6 Wind Tunnel Testing Matrix for RFSG Geometry. . . . . . . . . . . . . . 34

2.4 Mach 6 Wind Tunnel Testing Matrix for GHV Geometry. . . . . . . . . . . . . . . 43

3.1 CBAero Flow Analysis Conditions $\ldots \ldots \ldots$. . . . . . . . . . . . . . . 47

$4.1 \quad$ UNLATCH Run Conditions. $\ldots \ldots \ldots$. . . . . . . . . . . . . . . . . . . . . . . . . . . 83

4.2 Numeca .conf file components $\ldots \ldots \ldots$. . . . . . . . . . . . . . 90

$4.3 \quad$ HF1 boundary conditions from .mapbc file . . . . . . . . . . . . . . . . . . . . 90

4.4 Numeca quality criteria for HIFiRE-1 viscous volume mesh . . . . . . . . . . . . . 91

4.5 Restarts for HIFiRE-1 in Fun3d $\ldots \ldots \ldots$. . . . . . . . . . . . . . 93 


\section{LIST OF SYMBOLS}

\begin{tabular}{|c|c|}
\hline$a$ & Speed of sound \\
\hline$\alpha$ or $A O A$ & Angle of attack \\
\hline$C_{D}$ & Drag coefficient \\
\hline$C_{L}$ & Lift coefficient \\
\hline$C_{M}$ & Pitching moment coefficient \\
\hline$C_{p}$ & Pressure coefficient \\
\hline$I E$ & Internal energy \\
\hline$J$ & Flow functional \\
\hline$K E$ & Kinetic energy \\
\hline$M$ & Mach number \\
\hline$\hat{n}$ & Local surface normal \\
\hline$p$ & Pressure \\
\hline$P_{T}$ & Pressure tap \\
\hline$q$ & Dynamic pressure \\
\hline$R$ & Universal gas constant \\
\hline$R e$ & Reynolds number \\
\hline$T$ & Temperature \\
\hline$T_{w}$ & Temperature at the wall \\
\hline
\end{tabular}




$\begin{array}{ll}\vec{V} & \text { Velocity vector } \\ \vec{V}_{\text {surface }} & \text { Surface velocity vector } \\ \gamma & \text { Specific heat ratio } \\ \rho & \text { Density }\end{array}$

\section{Subscripts}

0

Total or stagnation condition

$\infty$

Freestream condition 


\section{CHAPTER I}

\section{INTRODUCTION AND BACKGROUND}

Since the beginning of flight, with the first airplane flight on December 17, 1903 by the Wright Brothers, the need to find ways to fly faster and farther have been the basis of aeronautics research. This can been seen through the exponential increase in both speed and altitude over the past 100 years of flight [7].

\subsection{Overview of Hypersonic Flow}

Hypersonic describes a flow regime in which properties of the flow field, that are explicitly dependent on one variable based on lower speed assumptions (or deemed constant), become dependent on multiple variables of the flow. This is seen in the real gas effects of equilibrium flow in which the specific heats are no longer constant and are dependent on temperature and pressure. In order to understand hypersonic flow, preconceived assumptions and generalizations about the flow must be looked at further to make sure the basic equations and their dependencies are valid at this higher Mach regime due to coupling effects that are much less common at lower speeds.

A more specific representation of what makes hypersonic flow unique can be seen in the diagram below where the concepts of thin shock layer, entropy layer, viscous interaction, velocity and temperature slip, and high temperature effects are presented. Unlike with subsonic and/or supersonic flow regimes, the various components of the aircraft (i.e. wing, fuselage, propulsion device) in hypersonics are viewed as integrated pieces and cannot be simply de-coupled during analysis. 
This is only one of the many differences between the various flow regimes and contributes to why analysis in the hypersonic regime becomes increasing complex.

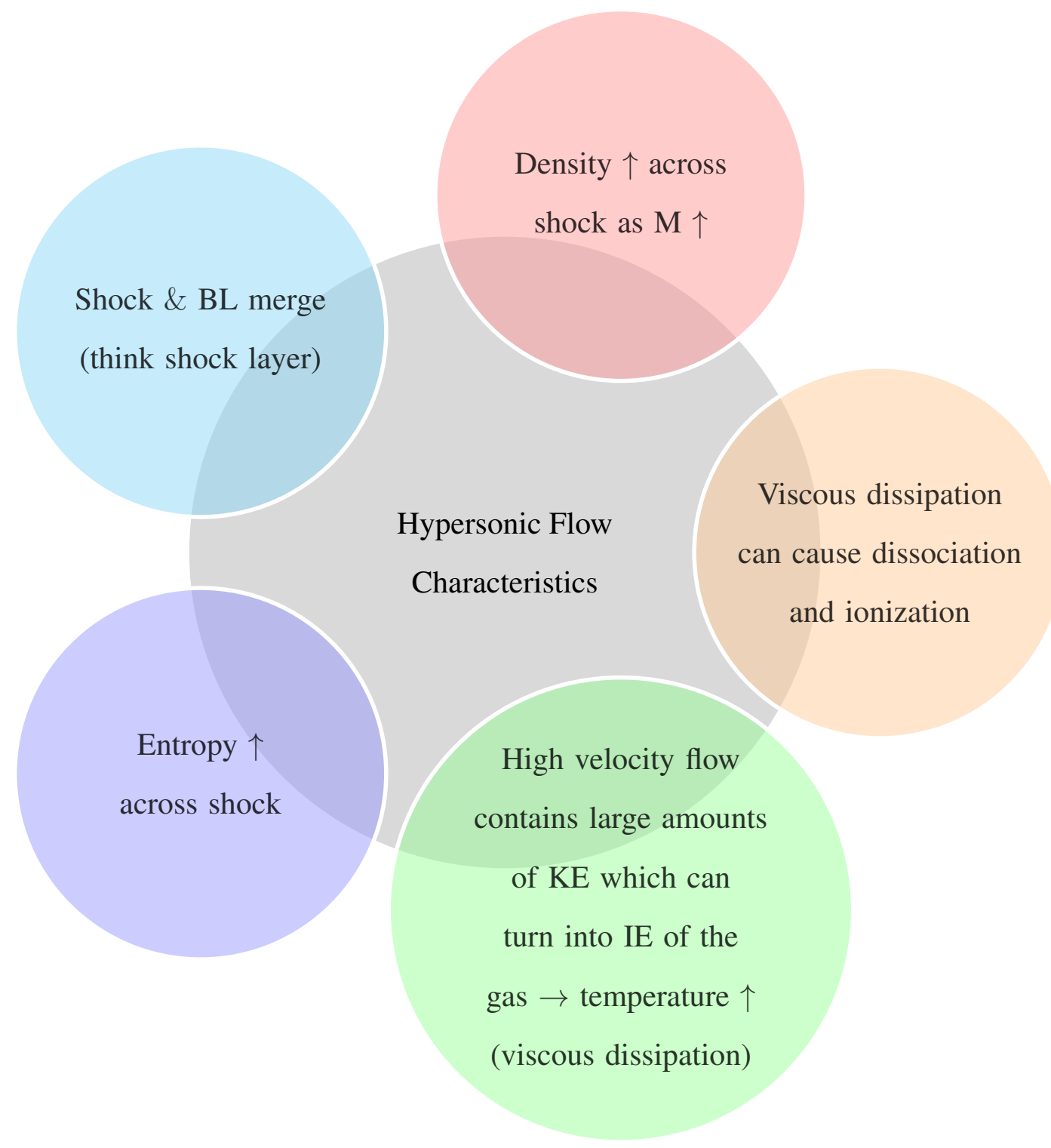

\subsection{Brief History of Hypersonics}

The evolution of hypersonic research can be dated back to as early as the the 1920's when Max Valier, who was a rocket enthusiast, recommended adding rockets to conventional aircraft. This spawned the first conceptual designs for hypersonic vehicles that came in the 1930's by the 
Austrian Eugen Sãnger and his wife Irene Bredt. Their space transporter design, named the "Silvervogel", featured a liquid fuel propulsion system, which practical use would not be seen until the development of the Space Shuttle decades later [1].

As shown below in Figure 1.1, the evolution of hypersonic testing, which began from the initial idea of placing rockets on conventional aircraft has seen many developments throughout the decades. The hypersonic revolution is comprised of both space and aeronautic testing, which have each guided the testing and design of each other throughout the quest to gain information as to the flow features experienced at such high speeds and altitudes.

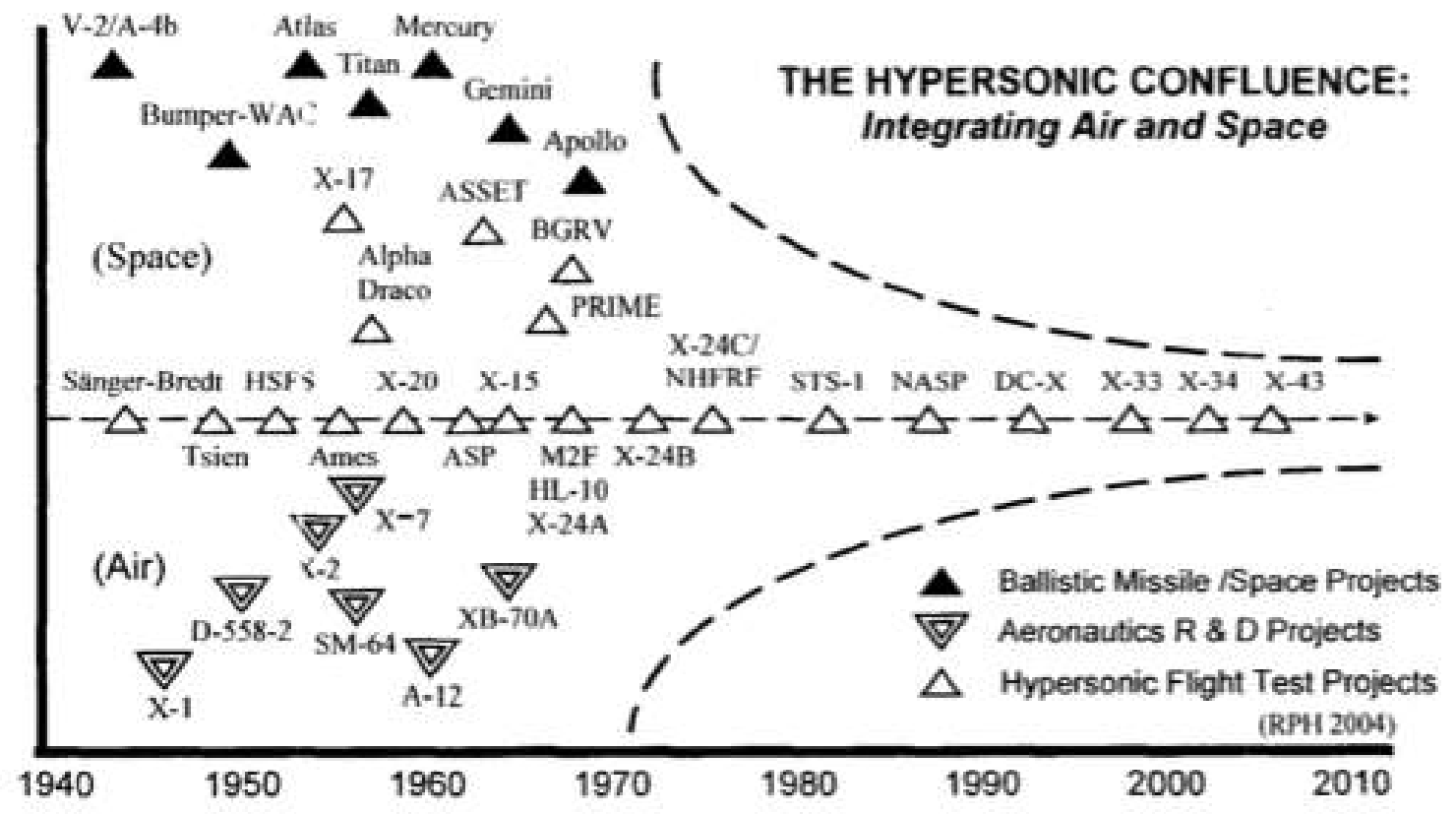

Figure 1.1: Hypersonic Program Timeline [1]. 


\subsubsection{The Birth of Hypersonics}

Early hypersonic accomplishments, as shown in Figure 1.1, laid the groundwork for future hypersonic studies. The A-4b, on January 14, 1945, was the first winged body, variant of the V2, to reach supersonic speeds and approach hypersonic speeds of $M=4$. Another V-2 variant, the Bumper-WAC, became the first vehicle to actually reach hypersonic speeds on February 24, 1949 , by attaining $M=7.6$. Finally, in efforts to gain data on reentry in order to safely get a warhead through the atmosphere, the X-17 booster reached $M=14.4$ on February 7, 1956 [1]. The importance of the first manned flight at supersonic speeds $(M=1.06)$, performed by Charles $\mathrm{E}$. "Chuck" Yeager on October 14, 1947 on the Bell XS-1, is significant in that it sparked a growing interest in quest for answers for the multitude of unknowns in hypersonic research. This impact is seen when Russian Yuri Gagarin becomes the first human to experience hypersonic flight while orbiting the earth and re-entering on April 12, 1961 aboard the Vostok I rocket [7].

\subsubsection{Major Breakthrough: The X-15}

The North American X-15 hypersonic research aircraft is one of the most accomplished and decorated hypersonic aircraft in history, spanning from 1959 to 1968 . Of the 199 X-15 missions, 13 met the United States Air Force requirement of exceeding an altitude of 50 miles, two of which qualified as space flights as an altitude of 100 kilometers (62.1 miles) was achieved. The aircraft was launched from a B-52 and by the end of its missions set the world's unofficial speed and altitude records of 4,520 mph (Mach 6.7) and 354, 200 feet respectively [8]. Contained in the nearly 700 technical reports attained from the program, valued insights into the aero-heating, structural demands, and pilot feedback from flying at such high speeds and altitudes were invaluable to the growth of hypersonic research in the mid-1900's. The X-15 aircraft can be seen in Figure 1.2 . 


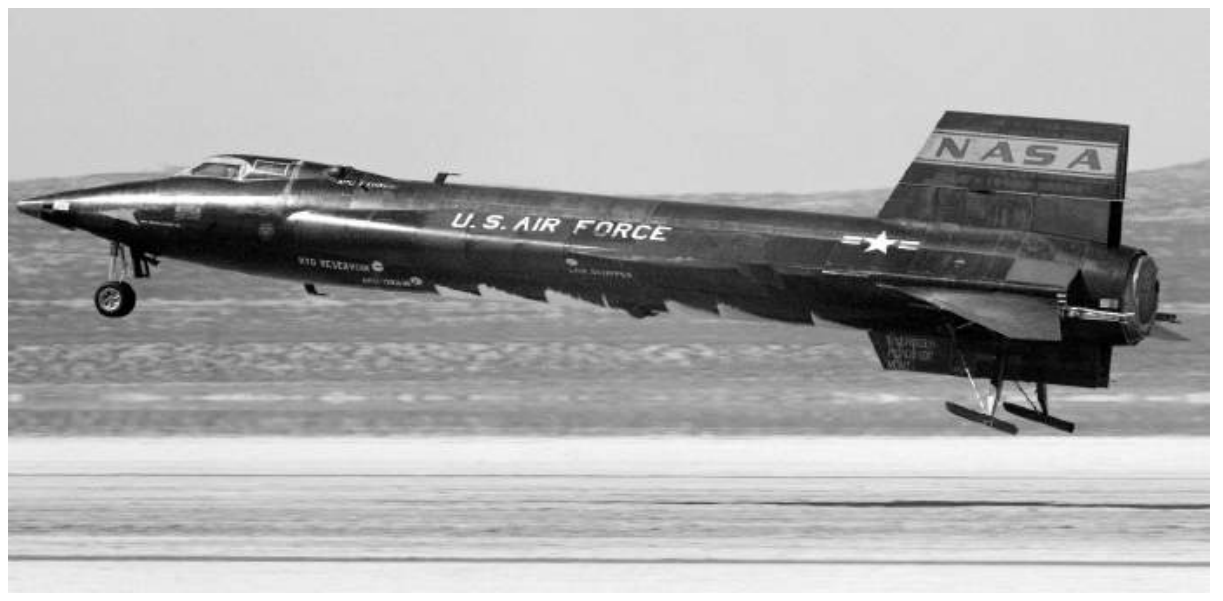

Figure 1.2: The X-15 Hypersonic Research Aircraft [2].

The first X-15 flight which took place on 8 June 1959 was unpowered to evaluate the gliding abilities as well as basic configuration of the aircraft [9]. On November 15, 1967, during X-15 flight 191 U.S Air Force test pilot Michael Adams was killed when the aircraft entered hypersonic spin on decent in which the airframe broke apart at 60,000 feet while experiencing up to $16 \mathrm{~g}$ 's. Major Adams was posthumously awarded Air Force astronaut wings for reaching an altitude of 50.4 miles above the earth's surface. Many significant achievements were made by the X-15 hypersonic research program that were later applied to numerous hypersonic vehicles such as the space shuttle [10].

\subsubsection{Propelling the Future: The X-51 Waverider}

The X-51A was a joint project by AFRL and DARPA stemming from the AFRL HyTECH program to test hypersonic propulsion techniques. The goal was to evaluate scramjet capabilities at hypersonic speeds that could not be completely verified with ground testing. The vehicle itself was built by Boeing with the JP-7 hydrocarbon fuel-cooled scramjet engine built by Pratt and Whitney. The X-51 was launched from a B-52 aircraft reaching Mach 6. The X-51A got coined "WaveRider" 
due to the fact that it uses the shock waves produced at high speeds to add compression lift, much like that of the XB-70 Valkyrie which used compression lift to surf on its own wake and reduce induced drag significantly. The X-51A's configuration supported a missile like geometry with an extremely large length to width ratio due to the large amounts of air that move through the scramjet at hypersonic speeds.

Initially four flight tests were to be conducted, with the first completed on 26 May 2010 which reached a speed of Mach 5 for over 200 seconds. Although it did not reach the goal of 300 seconds during the first test flight, the X-51A achieved its first successful flight on its fourth and final test run on 1 May 2013 where it reached a speed of Mach 5.1 and flew for a total time of over 6 minutes, which became the longest air-breathing hypersonic flight in history [11].

\subsection{Hypersonic Experiments}

At NASA Langley, the Aerothermodynamics Labratory (LAL) consists of three hypersonic blow-down wind tunnels ranging from Mach 6 to Mach 10. The most similar tunnel to that being used for this research at Wright-Patterson Air Force Base is the 20 inch Mach 6 air tunnel which specializes in the study of boundary layer and laminar-to-turbulent transition regions. Current research in this tunnel with regards to evaluating the aero-thermal response includes the use of temperature sensitive paint (TSP) along with a variety of other techniques such as two-color relative-intensity phosphor thermography. TSP allows for global surface temperature measurements to be made using a CCD array, similar to that of the two-color relative-intensity phosphor thermography but with the phosphor system designed specifically for high temperatures. The TSP technique used in the LAL facility where an oxygen impermeable layer is a clear urethane sealant in which the luminophore ruthenium trisbypyridine (Rubpy) is dissolved and applied over the white acrylic primer base coat on the models. The models are illuminated using near UV to blue wavelength light [3]. An example 
of TSP used to determine the effects of protuberance in the Mach 10 tunnel at the LAL is shown in Figure 1.3

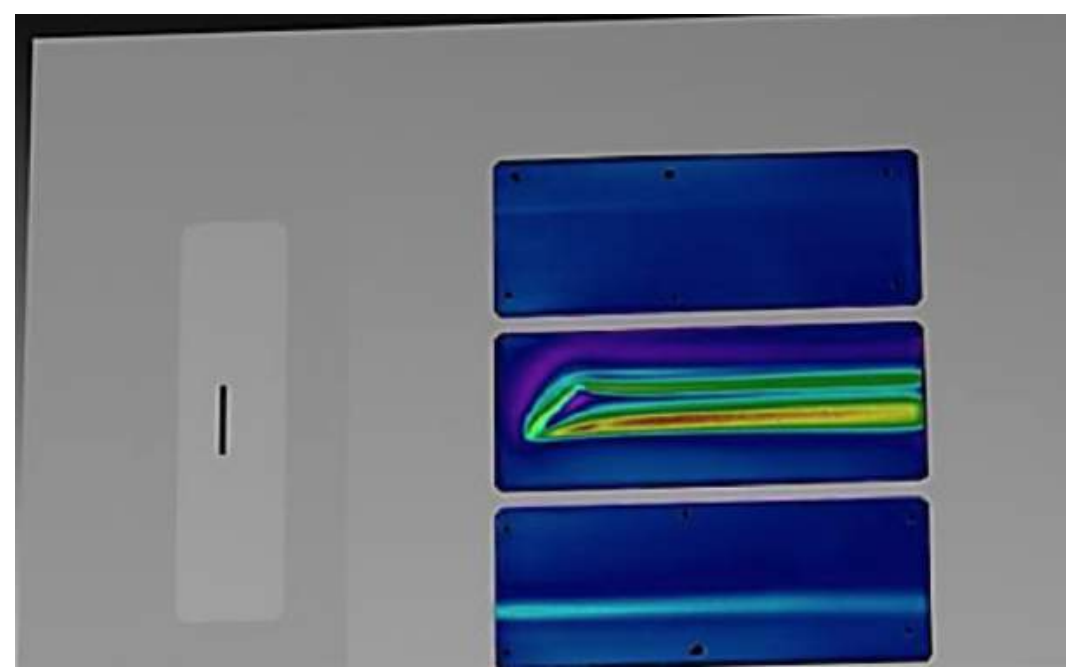

Figure 1.3: NASA Langley LAL Mach 10 tunnel TSP results [3].

The use of temperature sensitive paint (TSP) at supersonic speeds to determine the temperature distribution on various models was previously employed in the Transonic Gasdynamic Facility (TGF) at WPAFB in March of 2013. These tests were conducted on Oak Ridge National Laboratory (ORNL) test articles. These articles are similar to pitot tubes with both straight-cut and beveled-tipcut configurations, shown in Figure 1.4. The surface temperature distributions for both the beveled and straight-tip models at $M=3$ and $R e=4 \cdot 10^{6}$ are shown in Figure 1.5 [4]. 


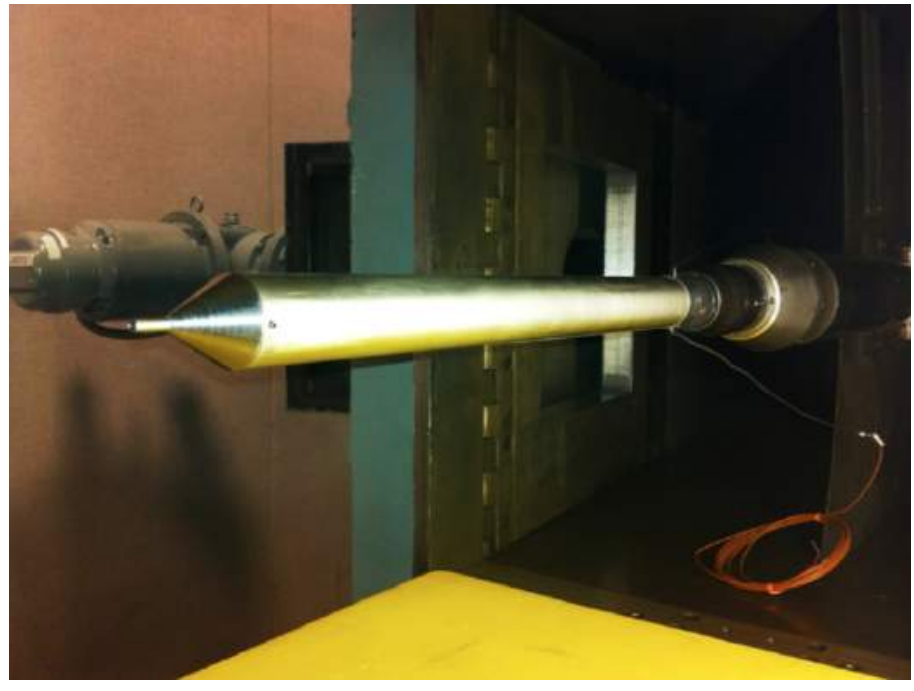

Figure 1.4: ORNL TSP test model in TGF tunnel [4].

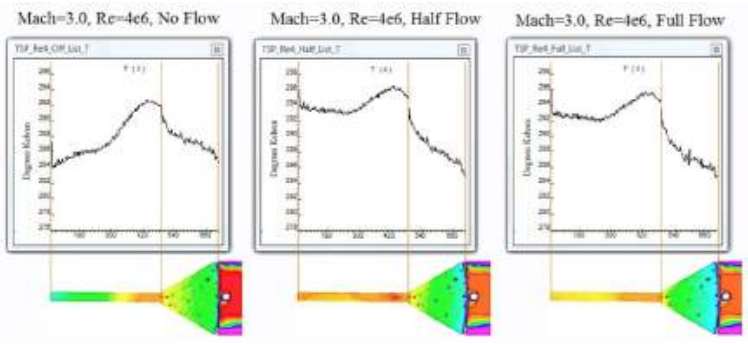

Beveled Tip

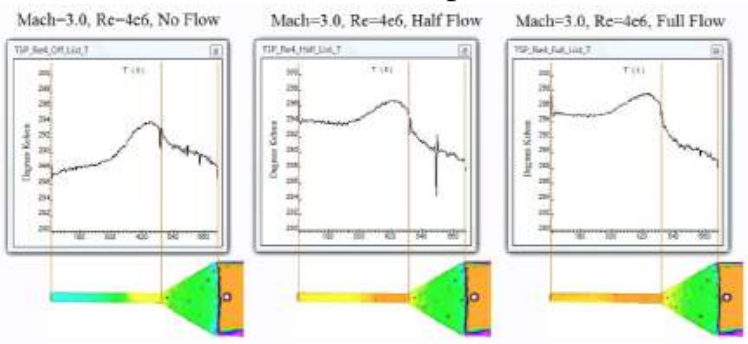

Straight Tip

Figure 1.5: TSP results for ORNL model configurations at $M=3$ and $R e=4 \cdot 10^{6}$ [4]. 


\subsection{Hypersonic Numerical Simulations}

Although experimental techniques can give accurate information about the flowfield, the cost of testing and issues with matching the hypersonic flow regime for some flight conditions lends numerical techniques to be used more prevalently in the future. Not only is solving the problem numerically cheaper than experimental or ground testing, the ability to have information for every point in the flowfield is valuable [10]. This is not to say the field of computational fluid dynamics (CFD) does not have its drawbacks as well. The amount of computing resources, time and money needed to model a transient, viscous flow for a complex geometry at a high fidelity (Direct $\mathrm{Nu}-$ merical Simulation) is not currently viable and is dependent on whether Moore's Law (number of transistors on a computer chip doubles every two years) can hold. For decreasing the fidelity and thus cost of the simulations the turbulence in the flow has to be modeled. However, this is a significant area of concern and interest for the CFD community to truly understand what turbulence is and how to model it accurately.

\subsection{Objectives and Accomplishments of this Research}

The ability to predict and test surface temperatures at hypersonic conditions in the early design stages can have a significant impact on the success of a design. This research aimed to gain confidence in the ability to obtain aero-thermal data from the Mach 6 High Reynolds Number Facility at Wright-Patterson Air Force Base (WPAFB). After being dormant for nearly 20 years due to lack of funding for hypersonic testing facilities, a recent resurgence in need for such facilities, as the field of hypersonics became more prevalent, spawned the Mach 6 wind tunnel to be re-opened once again. With this re-opening, the window for submitting tests plans for the tunnel opened as well. With there being a large gap in time between testing in the Mach 6, the capabilities of the tunnel had to be re-assessed as confidence in the tunnel's capabilities was unknown. 
With this opportunity, Barry Hellman, Aerospace engineer with the Air Force Research Laboratories Hypersonic vehicles division (AFRL/RQHV), determined that an assessment of the aerothermal capabilities of the tunnel using temperature sensitive paint (TSP) could be of benefit to both the Air Force and to the experimental community. Having never used TSP in the Mach 6 tunnel before, the need for this assessment was deemed plausible and the project was awarded the funding to test such capabilities.

\subsubsection{Objectives}

The initial objectives of the research were as follows, shown in order of priority to be determined by the research.

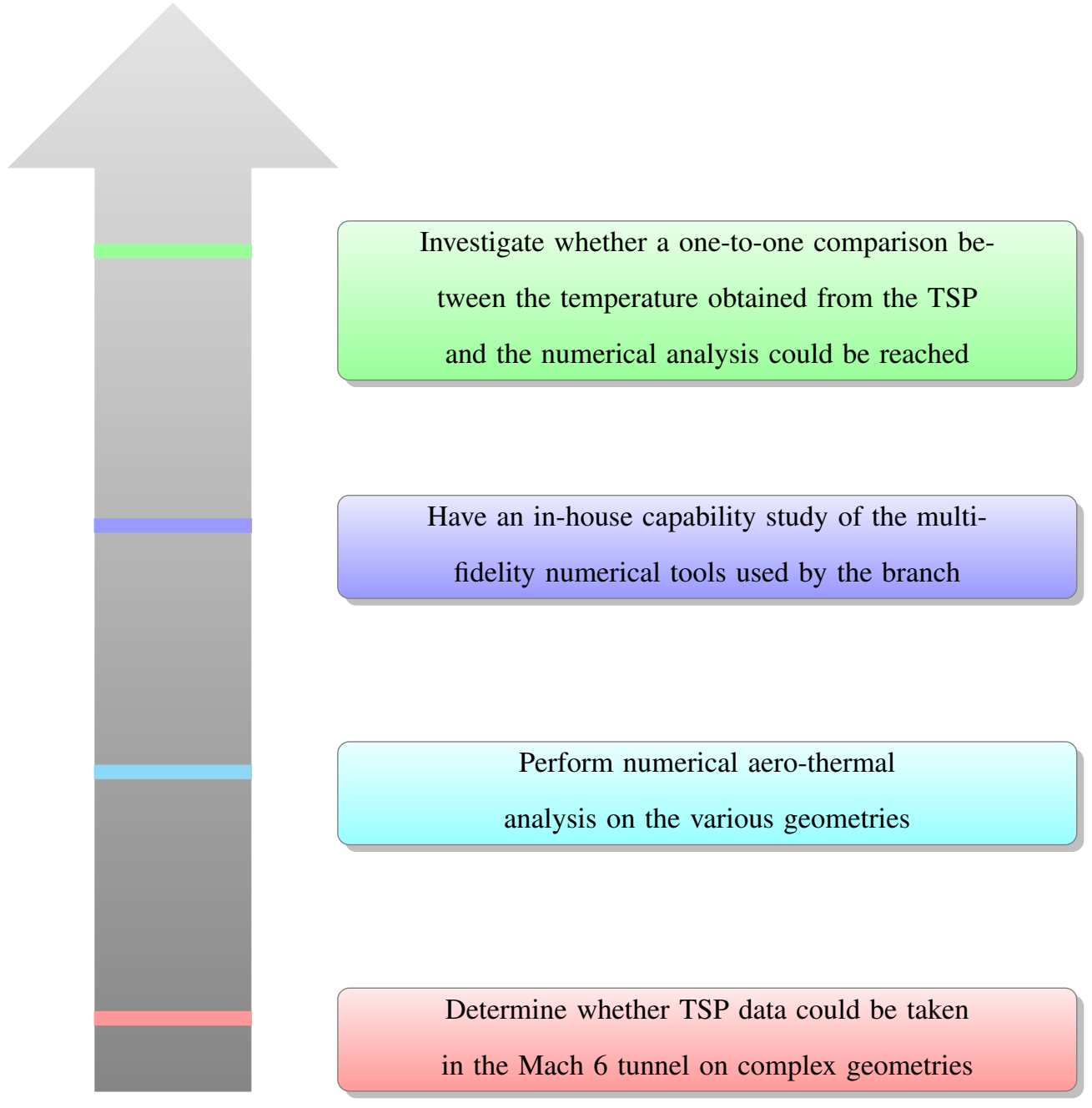




\subsubsection{Accomplishments}

Although not all of the objectives were met, namely the one-to-one comparison between the temperature obtained using TSP in the Mach 6 tunnel and the numerical analysis, the research did yield information that should be useful to the hypersonic and experimental communities. Below is an outline of the accomplishments of this research:

- Data for all three models using TSP, pressure taps and thermocouples were obtained from the Mach 6 tunnel

- Numerical analyses using up to three levels of fidelity for all three models were obtained and post-processed

- Capability study was performed to gauge the various fidelity levels ability to predict pressure coefficients at hypersonic conditions with validation from experimental testing

- Limited evaluation of aero-thermal capabilities of available numerical tools was conducted for all three models

- Recommendations for changes are given that need to be made to model construction and test setup in order to accurately judge whether the Mach 6 tunnel is producing valid aero-thermal data using TSP 


\section{CHAPTER II}

\section{MACH 6 WIND TUNNEL EXPERIMENTAL RESULTS}

The experimental testing for this research took place in the Mach 6 High Reynolds number facility located at Wright-Patterson Air Force base. The testing began in June 2016 and concluded in December 2016. There were numerous delays in the testing due to issues with the tunnel, which caused the testing to span nearly seven months. Jim Hayes and Servane Altman were in charge of running the wind tunnel and were instrumental in the experimental testing process.

Beginning in August 2015, decisions on the design of the models, instrumentation location and testing procedure were decided upon by members of the team. Since testing with the TSP in the Mach 6 has been never done before, there were misunderstandings between the experimental team, the numerical team and the project manager that led to some of the shortcomings of the project outlined in Chapter VI. The main issues were what is the best way to compare experimental values for the surface temperature with the numerical analysis and whether force and moment data would be obtained from the experimental testing. In hindsight, the best way to have a one-to-one comparison between the experimental surface temperature with TSP and the numerical analysis would have been to make a true "thermal model" to be able to take surface temperature measurements and relate them to the heat transfer experienced within the model. In order to build a "thermal model" considerations for how the structural model was being built, in terms of thermocouples placement, wing thickness and material choices would have had to be taken into account. 
Surface temperature proved to be a difficult element to compare to the numerical analysis due to the fact that it is dependent on multiple factors that were not taken into account in the computational models, mainly conduction through the models surface due to thin model sections and the models not reaching the equilibrium temperature over the limited testing time the TSP could withstand.

\subsection{The Mach 6 Tunnel}

All experimental testing for this research was conducted in the Mach 6 High Reynolds Number Facility at WPAFB seen in Figure 2.1. A rapid decline in funding for hypersonic research caused the Mach 6 tunnel to become dormant for nearly 20 years. In 2009, plans for re-activating the Mach 6 tunnel were started with a need for hypersonic testing facilities stated as a "top 5 concern" by the Air Force [5]. With the tunnel finally running in 2014, the desire to determine the capabilities of the tunnel arose. One of these tests was this project, to determine whether the Mach 6 tunnel could be used to obtain aero-thermal data using temperature sensitive paint (TSP). A secondary goal is to determine how well these thermal measurements match numerical models as well as having an in house numerical comparison between various levels of fidelity computational tools. The test section conditions for the Mach 6 tunnel are given in Table 2.1. An external layout of the Mach 6 tunnel is shown in Figure 2.2, which outlines the setup Mach 6 tunnel components. Not shown in the layout are the two compressor units, which each house 4 compressors. 


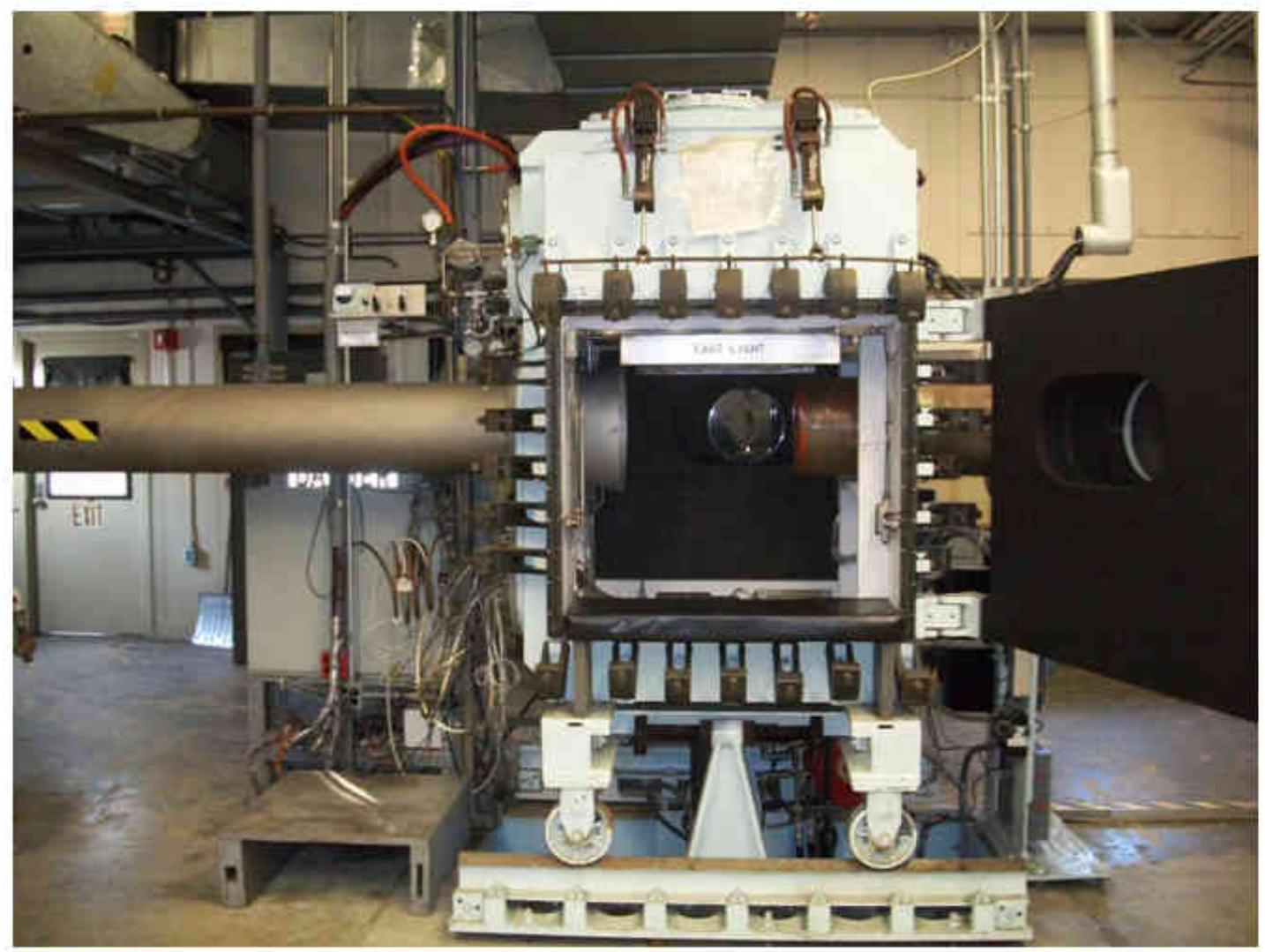

Figure 2.1: Mach 6 High Reynolds Number Wind Tunnel at Wright-Patterson Air Force Base [5].

Table 2.1: Mach 6 Wind Tunnel Testing Conditions [5].

\begin{tabular}{c|c} 
Test Section & 12 in. diameter open jet, 17 to 28 inches long \\
\hline Mach Number & $6.0(5.85$ measured during facility calibration $)$ \\
\hline Temperature Range & 440 to 640 degrees $\mathrm{F}$ \\
\hline Reynolds Number Range & 10 to 30 million/ft. \\
\hline Run Time & Continuous 5 minutes at $2000 \mathrm{psi}$ \\
\hline Density Altitude & Simulates flight from $30,000 \mathrm{ft}$ to $60,000 \mathrm{ft}$. \\
\hline Stagnation Temperature Range & 900 to 1100 Rankine \\
\hline Stagnation Pressure Range & 700 to 2100 psia - exhausting to atmosphere
\end{tabular}




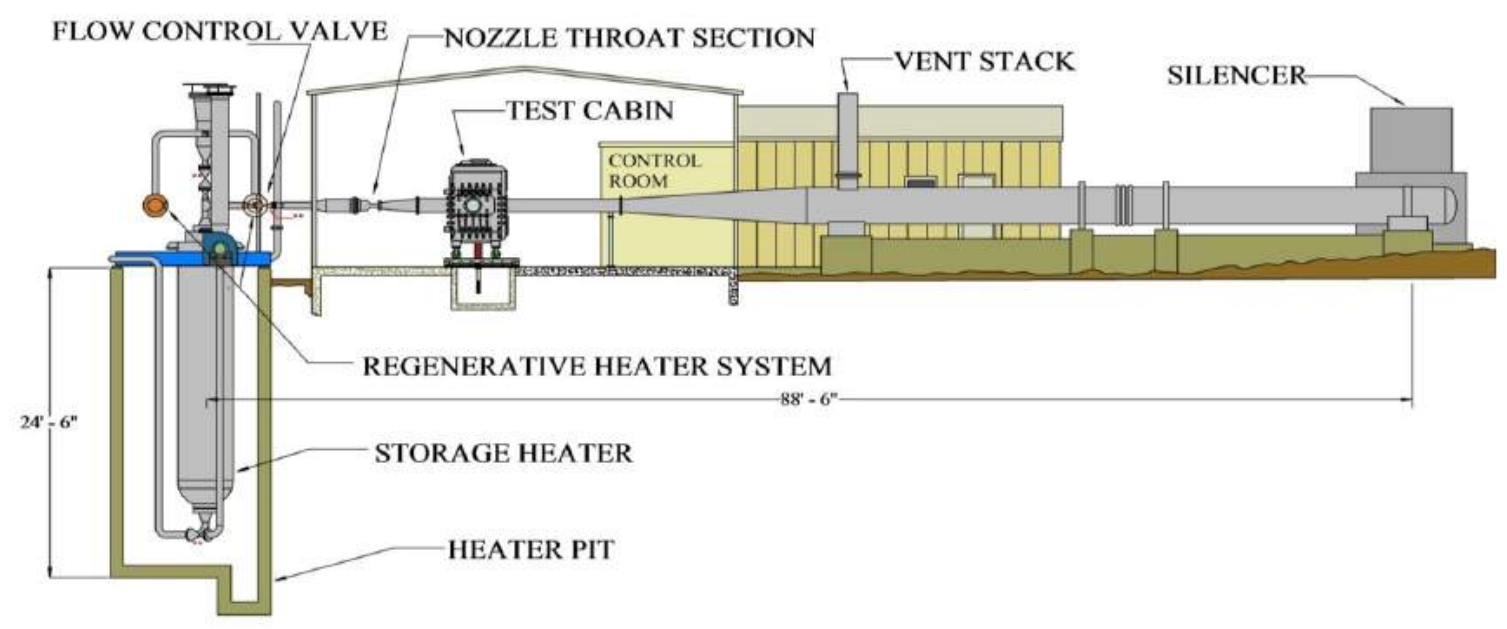

Figure 2.2: Mach 6 Wind Tunnel Layout [5].

\subsubsection{Initial Paint Adhesion Testing}

In order to determine the appropriate roughness, to insure proper paint adhesion, pressure sensitive paint (PSP), which has very similar adhesion capabilities to the temperature sensitive paint (TSP), was initially used in testing. Two models were evaluated to test appropriate surface roughness, one sharp and one blunt nose cone that mirror the HIFiRE-1 model except without instrumentation. The two test models can be seen in Figure 2.3, which have a fine glass bead finish with a roughness of $32 \mathrm{RA}$. This roughness was chosen due to proper paint adhesion at this roughness using flat plate models, although how the paint would adhere on more complex geometries with increased curvature was unknown. Schlieren images were taken for the sharp and blunt roughness nose models to get a qualitative look at the shock structure for future model construction and numerical analysis, shown in Figure 2.4. Gaining insight into the shock structure allows for possible shock boundary-layer interaction (SBLI) to be seen, which could raise concerns about the placement of the instrumentation. The schlieren imaging also served as a guideline for the meshing strategy for the numerical analysis. 


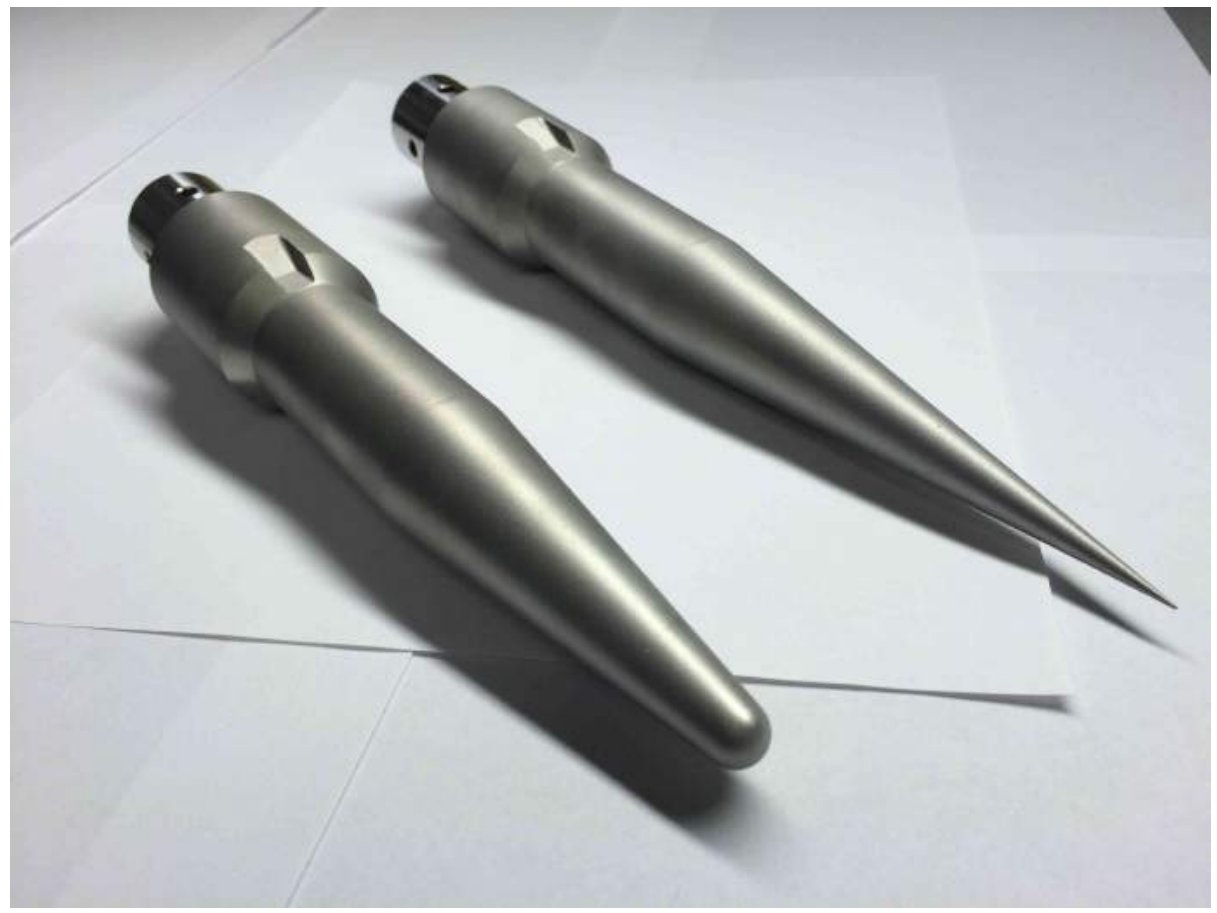

Figure 2.3: Paint Adhesion testing models 

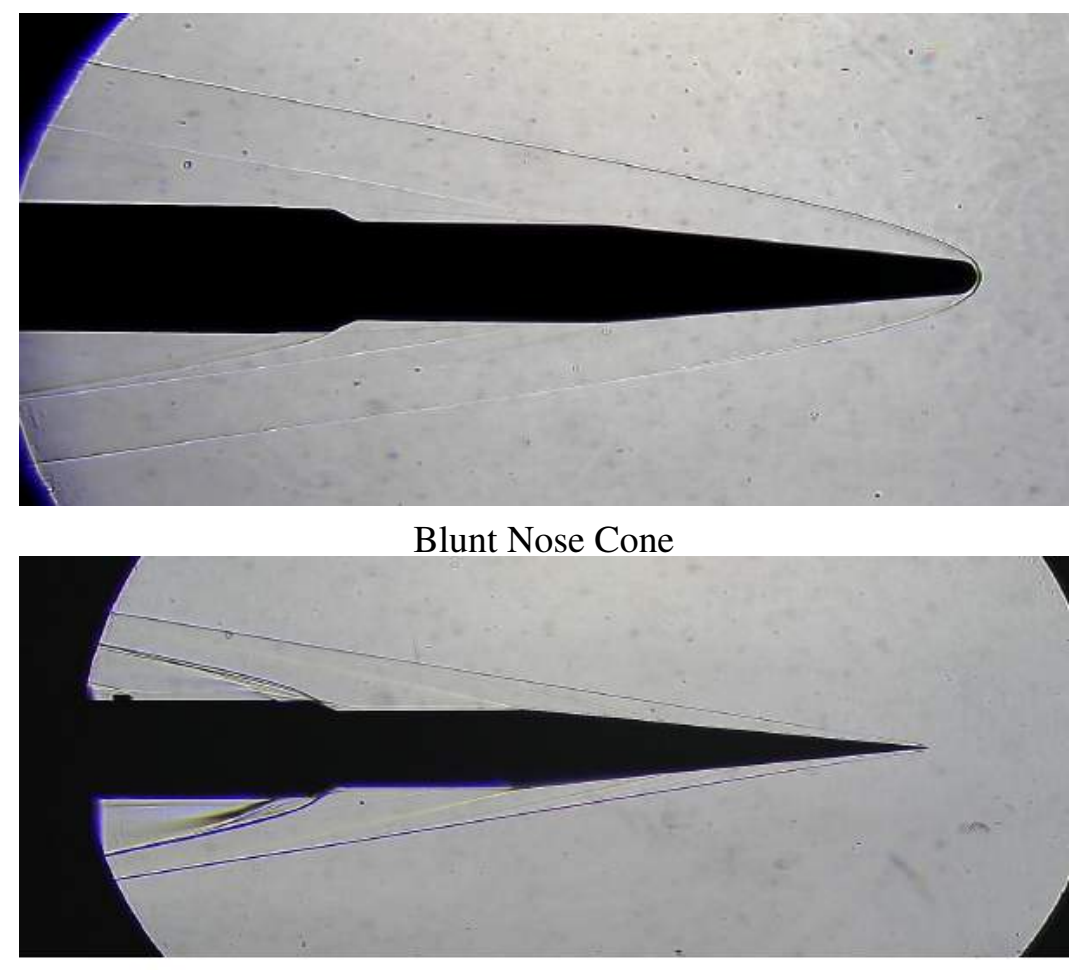

Sharp Nose Cone

Figure 2.4: Schlieren images from roughness cone models testing at $\alpha=0$ in Mach 6 tunnel

\subsection{Geometries}

The three geometries chosen for benchmarking are the RFSG reusable booster system, the generic hypersonic vehicle (GHV), and the HIFiRE-1 which are all shown in Figure 2.5. The goal of this research is to assess the aero-thermal capabilities of the Mach 6 wind tunnel for complex geometries. All three of these models exhibit unique challenges in both the TSP paint testing as well as the numerical analysis. The thermal contours gained from the TSP can give insight into the thermal response of bodies in Mach 6 flow, which in turn can inform subsequent structural analyses of future design iterations. If performed early in the design cycle, insights from the measurements 
may be able to guide designers to avoid unacceptable outer moldline (OML) decisions that can hinder success of the design later in the design process.

All models were constructed out of 17-4 Stainless Steel due to its high strength and relatively low price and availability. Rebecca Hoffman, with the University of Dayton Research Institute, was in charge of model design, instrumentation location and stress analysis [12].

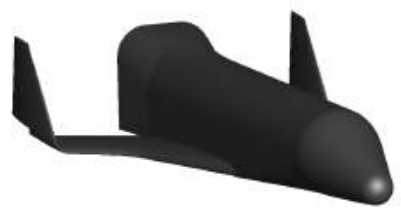

Reusable Booster System

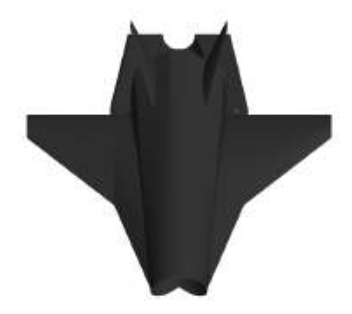

Generic Hypersonic Vehicle

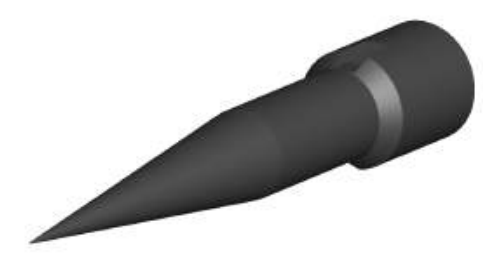

HIFiRE 1

Figure 2.5: Rendered geometries to be tested in Mach 6 High Reynolds Number Wind Tunnel [6].

\section{HIFiRE-1}

The Hypersonic International Flight Research Experimentation (HIFiRE) program is a bi-lateral hypersonic flight test program between the Air Force Research Laboratory (AFRL) and the Australian Defense Science and Technology Group (DTSG), formerly the Australian Defense Science and Technology Organization (DSTO). HIFiRE Flight 1 took place on March 22, 2010 to measure boundary layer transition and shock boundary layer interaction at Mach 7 flight conditions [13].

This model had 3 pressure taps and 2 thermocouples, which are shown in Figure 2.6. This model was built out of 17-4 stainless steel and in two pieces to allow for the instrumentation to be fed back through the sting. Figure 2.7 exhibits the dimensioned HIFiRE-1 wind tunnel model to be used in this research. The actual wind tunnel model can be seen in Figure 2.8 mounted in the 
Mach 6 tunnel. This model mimics the sharp nose cone used in the paint adhesion testing shown in Section 2.1.1. This model was tested with the notch both at 0 and 90 degree orientation from centerline. The results from the notch at 90 degree orientation were the ones of interest due to the resulting instrumentation being on the centerline and having the most variation with change in angle of attack.

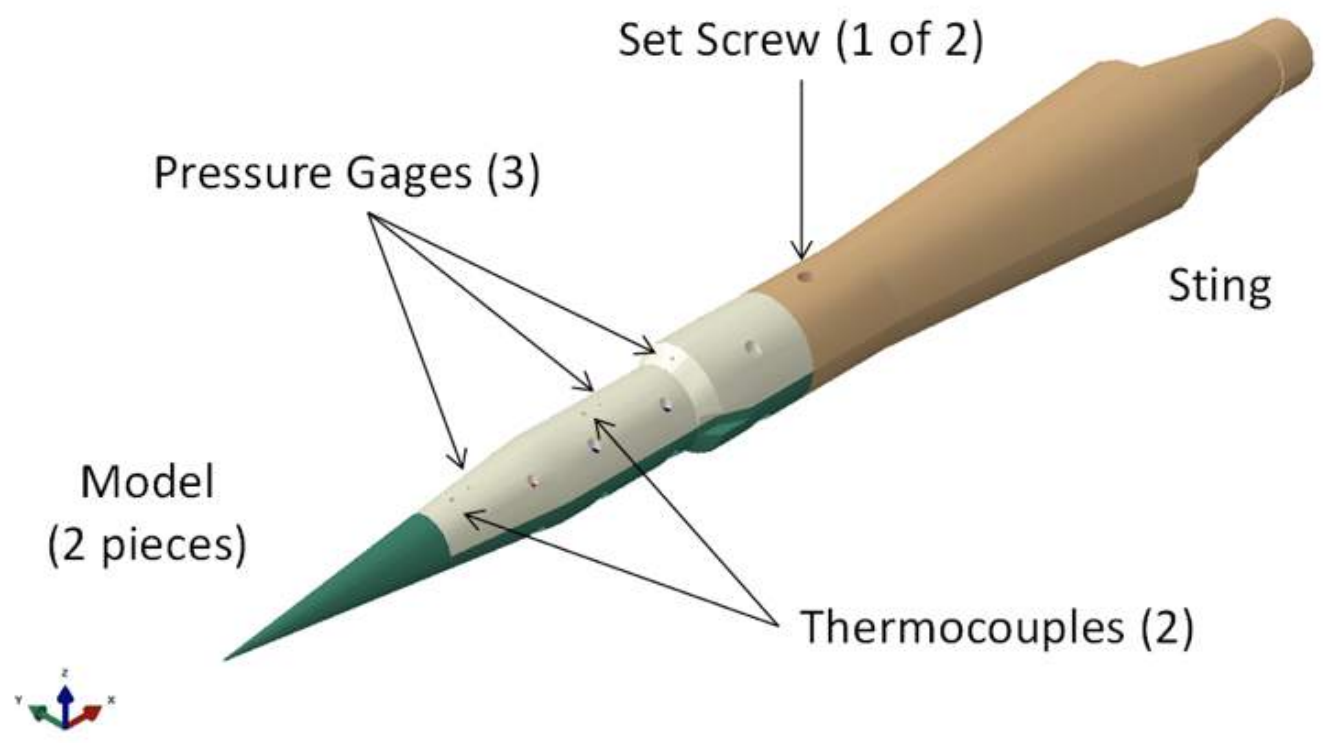

Figure 2.6: HIFiRE-1 rendered CAD model with stingmount. 


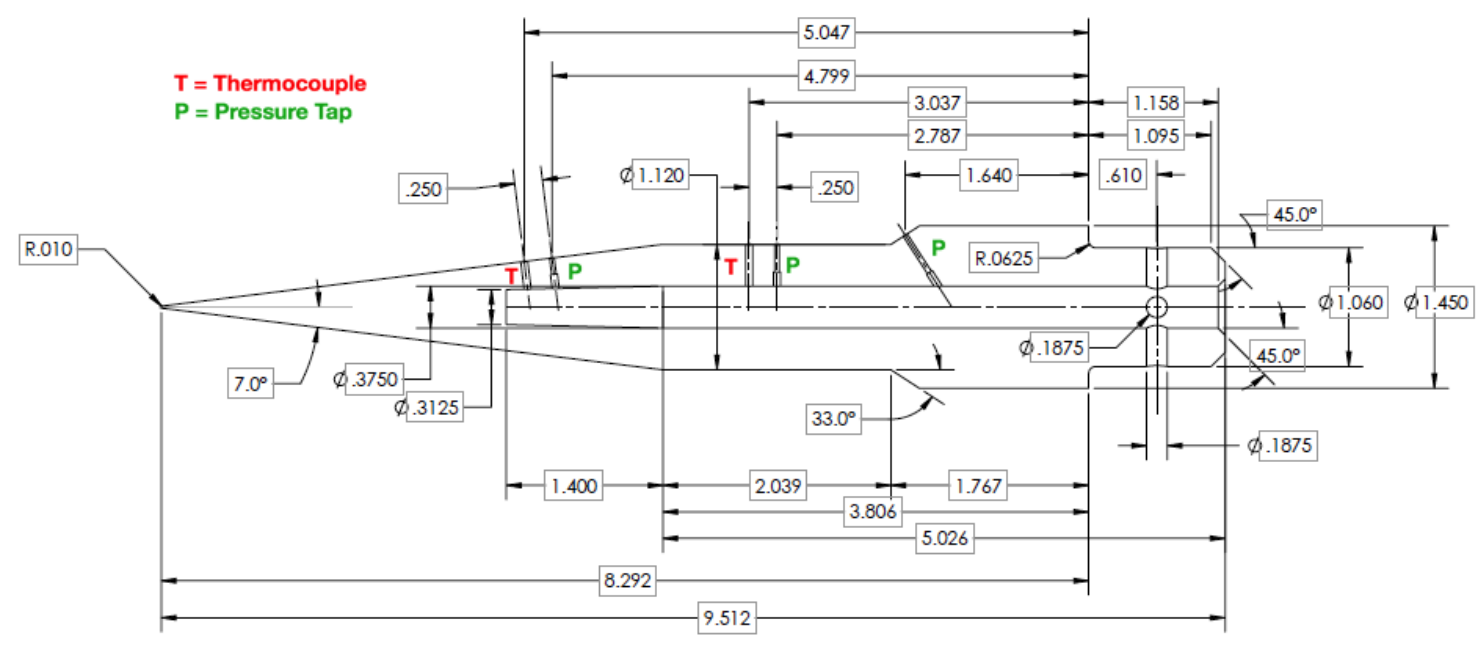

Figure 2.7: HIFiRE-1 instrumentation layout.

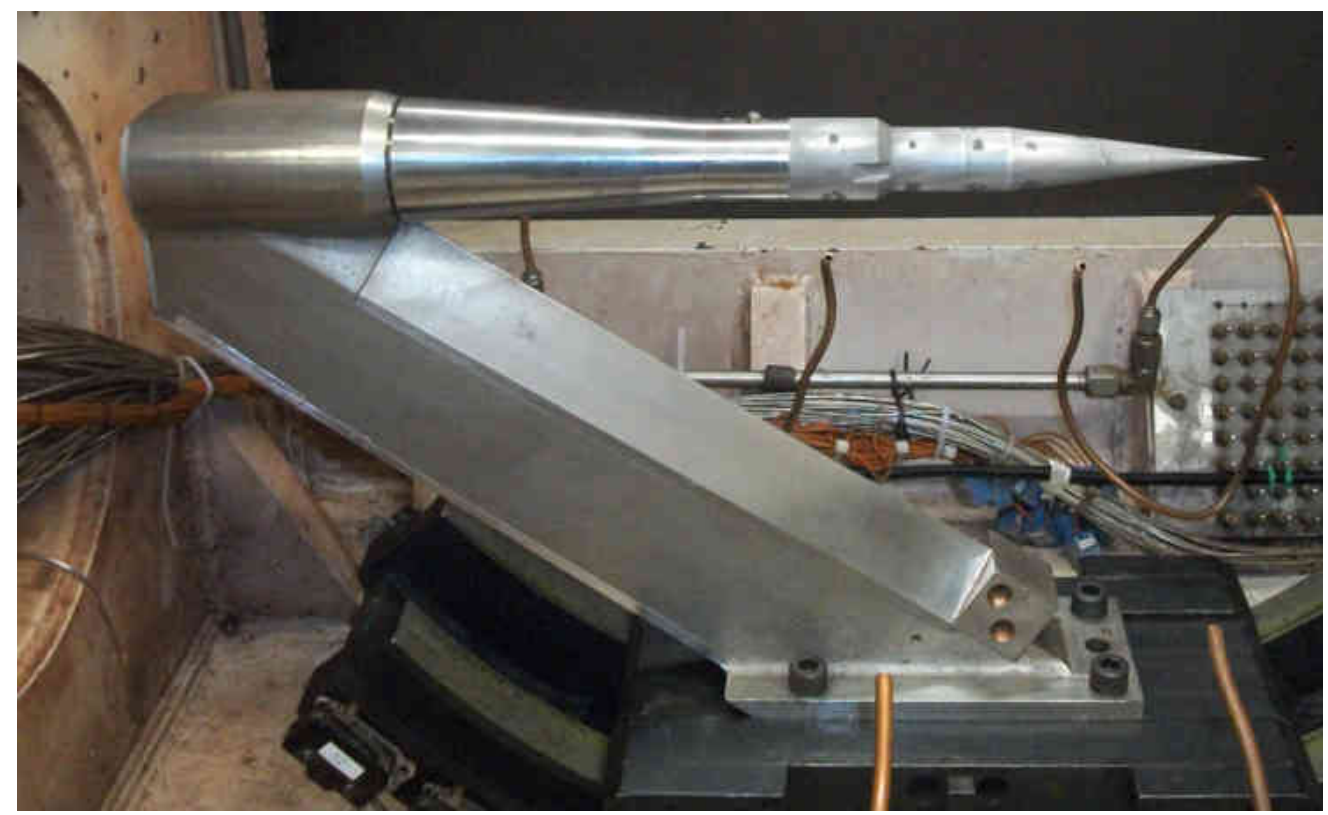

Figure 2.8: HIFiRE-1 unpainted model mounted in Mach 6 Wind Tunnel. 


\section{RFSG}

The Reference Flight System model G (RFSG) was developed under the AFRL Future responsive Access to Space Technologies (FAST) program. This vehicle design was developed based on lessons learned from the FAST program's three ground technology development efforts. This vehicle was designed as a reusable first stage which would be paired with an expendable upper stage to provide responsive and affordable space access. This model was chosen for this research because of the extensive aerodynamic database compiled for this model as well as its unique geometric features such as the wing-body combination and wing tip vertical fins.

An instrumented drawing of the RFSG geometry as well as the wind tunnel model itself can be seen in Figures 2.9 and 2.10, respectively. As can be inferred the model is approximately 9 inches long, not including the sting mount in the rear. This model features 8 pressure taps and 3 thermocouples, as indicated in Figure 2.9. The pressure tab at the nose had to be EDM lasered in due to the difficult angle for the instrument location but was deemed necessary to be able to capture the stagnation pressure at the nose.

In order to feed the instrumentation wiring to the back sting and to the data acquisition system, the RFSG model was built as two interlocking pieces, shown in Figure 2.11. There are 10 fastener holes and two dowel locations to help fasten the model together after instrumentation. 


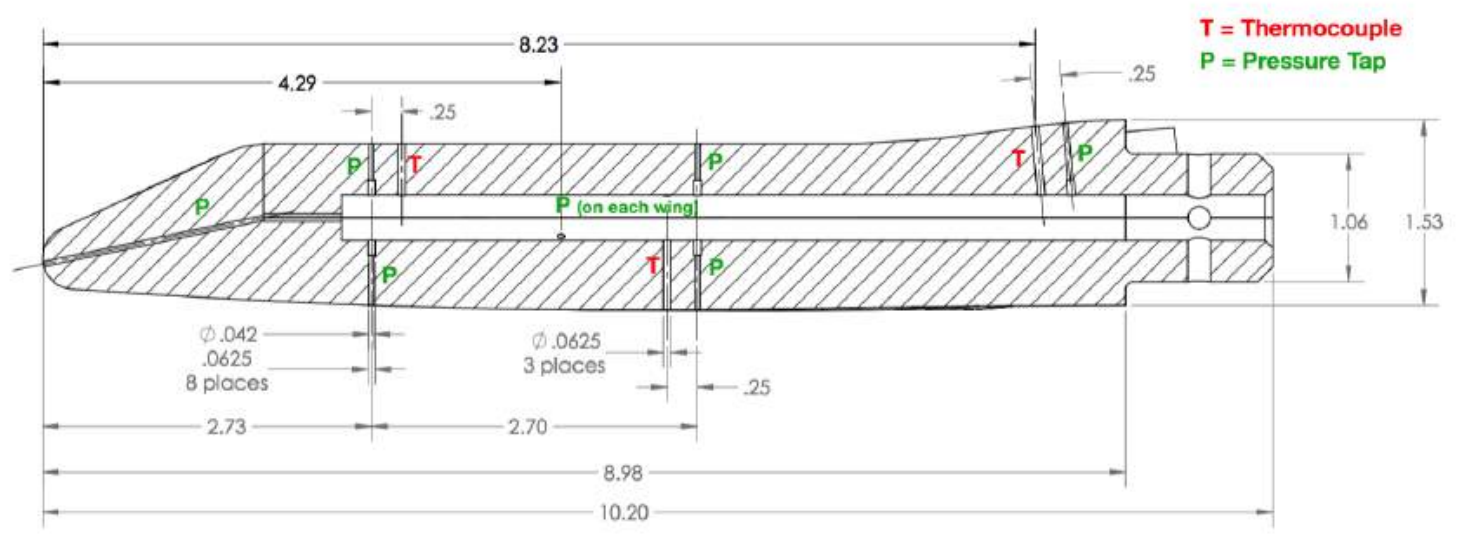

Figure 2.9: RFSG wind tunnel model with instrumentation.

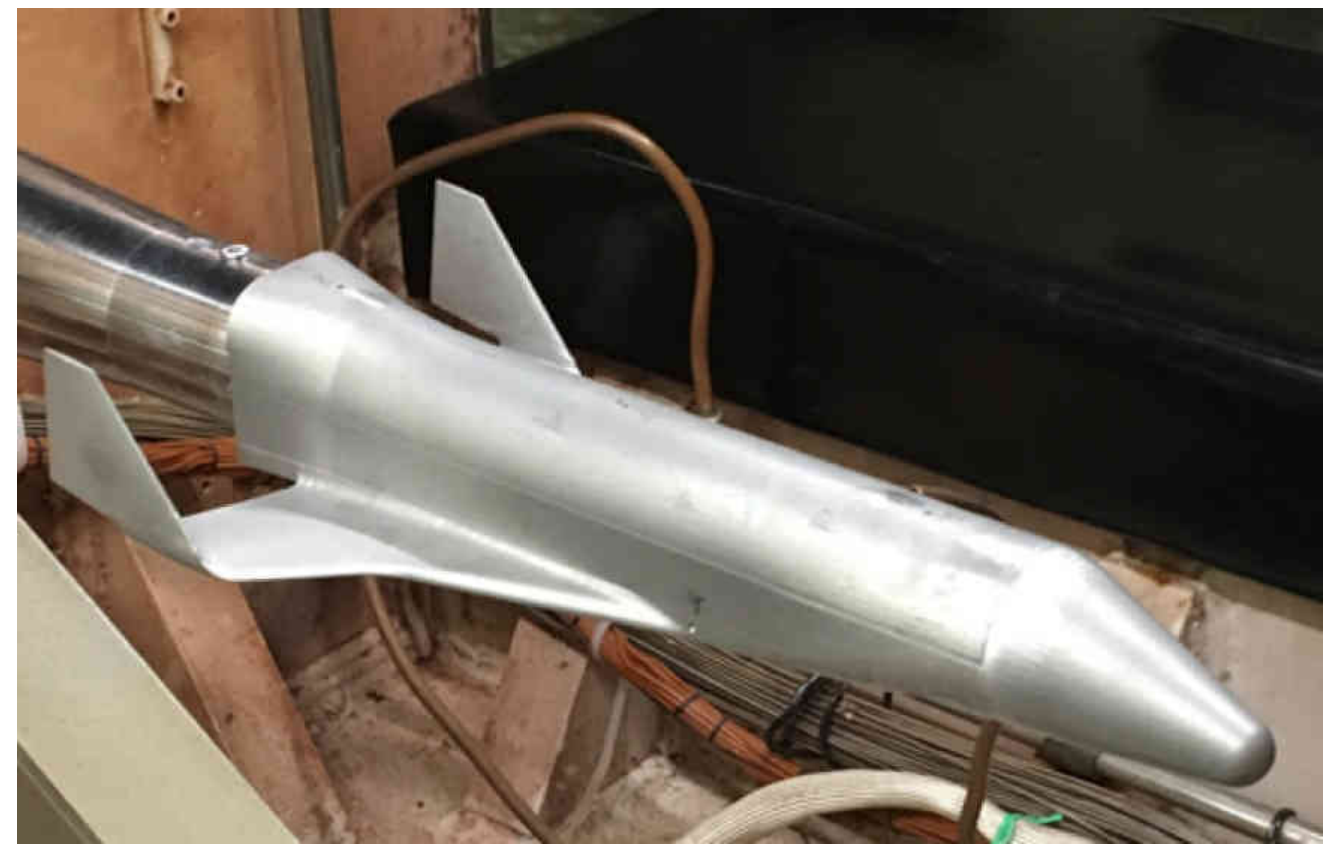

Figure 2.10: Unpainted RFSG model mounted in Mach 6 Wind Tunnel. 


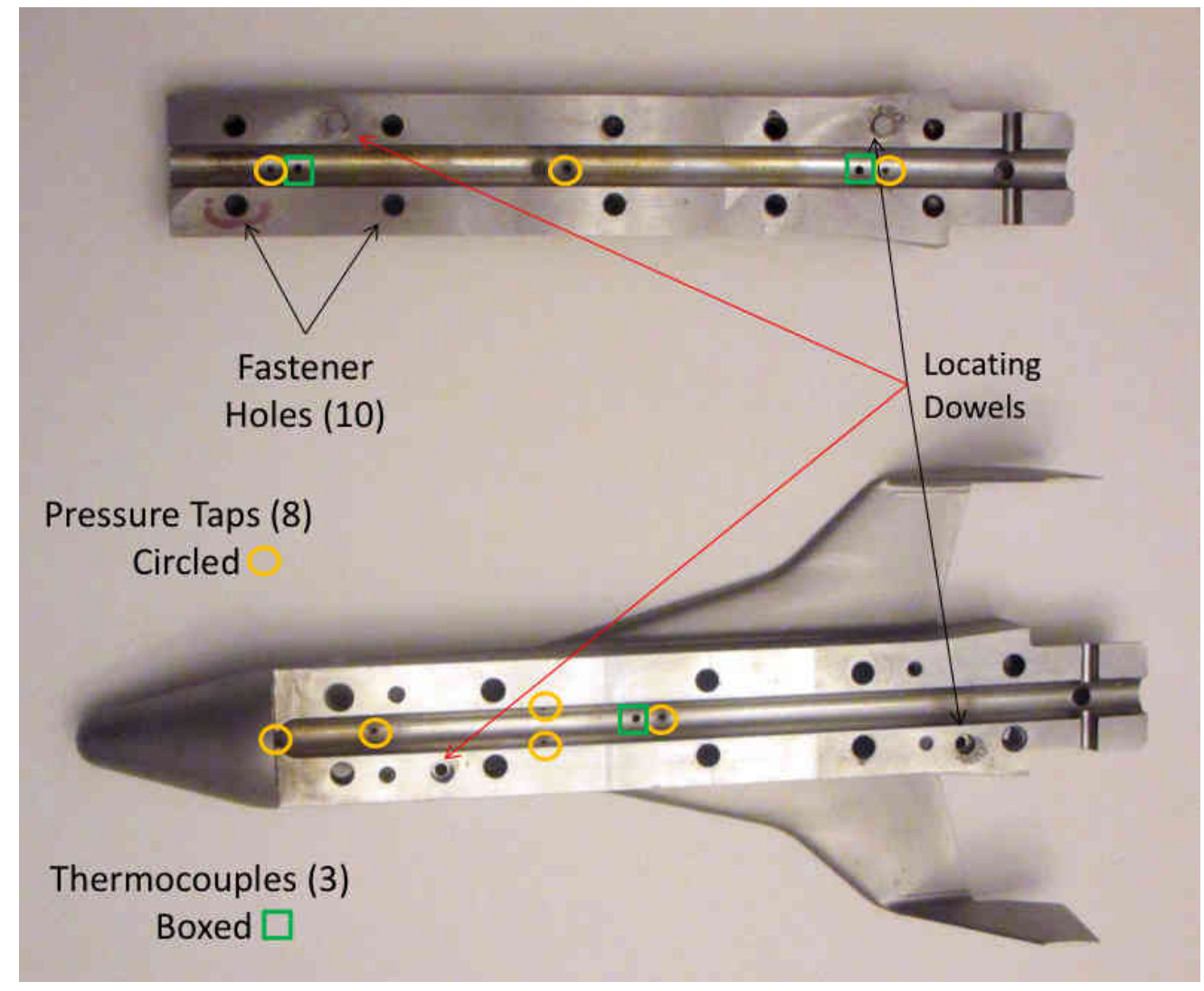

Figure 2.11: RFSG two-piece model sections.

\section{Generic Hypersonic Vehicle}

In order to foster collaboration between the government and other hypersonic stakeholders, AFRL created a family of Generic Hypersonic Vehicles (GHV) that are publicly released. This allows for data to be more easily disseminated within the hypersonic community in order to advance the field. The GHV is a slender, low aspect ratio wing-body configuration enclosing a supersonic combustion ramjet (SCRAMjet) engine with an inward-turning inlet. The vehicle is conceptually designed to have a metallic structure with a thermal protection system capable to withstand Mach 6 cruise conditions [14]. The model was chosen for its complexity as well as its ease of distribution within the community. Although the thermal response of the internal flow path could not be 
assessed, the inlet and exhaust regions are of major interest with this model and were made sure to be in sight of the camera when the TSP images were captured.

The GHV model used for this research had an added stingmount to allow for compatibility with the sting available in the Mach 6 tunnel. The stingmount adaptation to the GHV was incorporated in the computational modeling for this research as well, at least up until the instrumentation location. The top and side views of the model with instrumentation are shown in Figure 2.12. One of the major difficulties with this model was finding a location for the instrumentation. Due to extremely thin sections everywhere in the model, the eventual resulting instrument location was the only feasible option for this model. The GHV model mounted in the Mach 6 tunnel can be seen in Figure 2.13.

Due to the complexity of the model, mainly with the flow path, this model was 3-D printed out of 17-4 stainless steel. The model, as shown in Figure 2.14, was printed in three pieces which were fastened together. The final portion of the stingmount, shown as the portion without paint in Figure 2.28, was machined in order to save cost and was laser welded to the 3-D printed portion. 


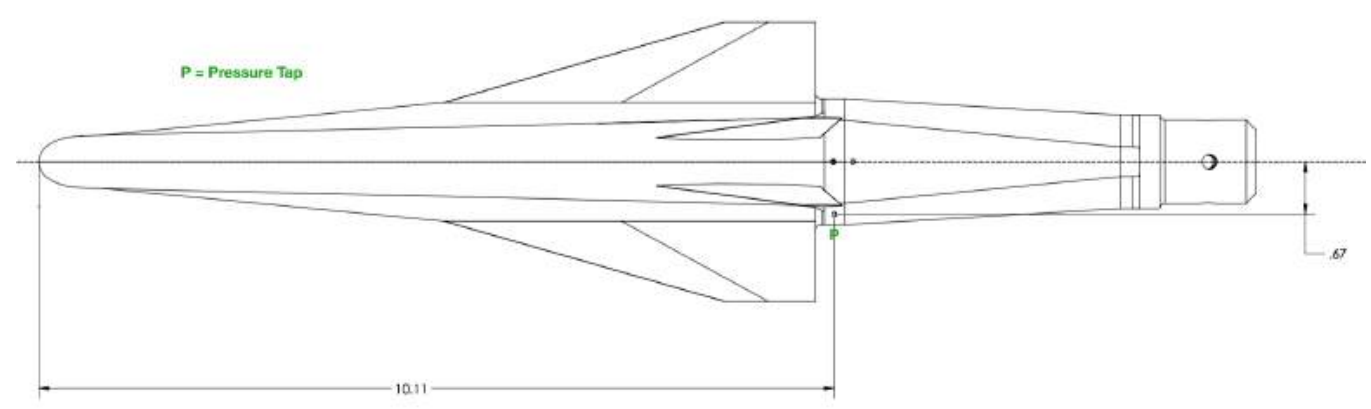

Top view

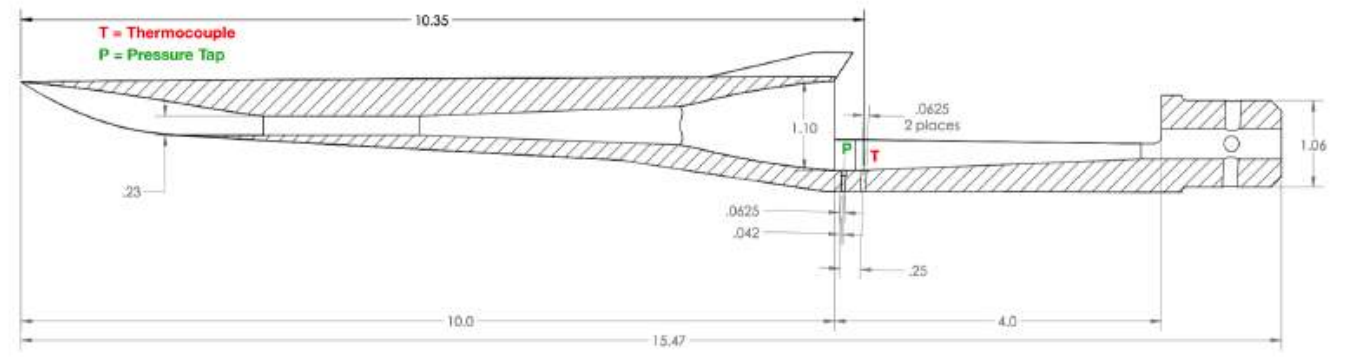

Side view

Figure 2.12: GHV wind tunnel model with instrumentation.

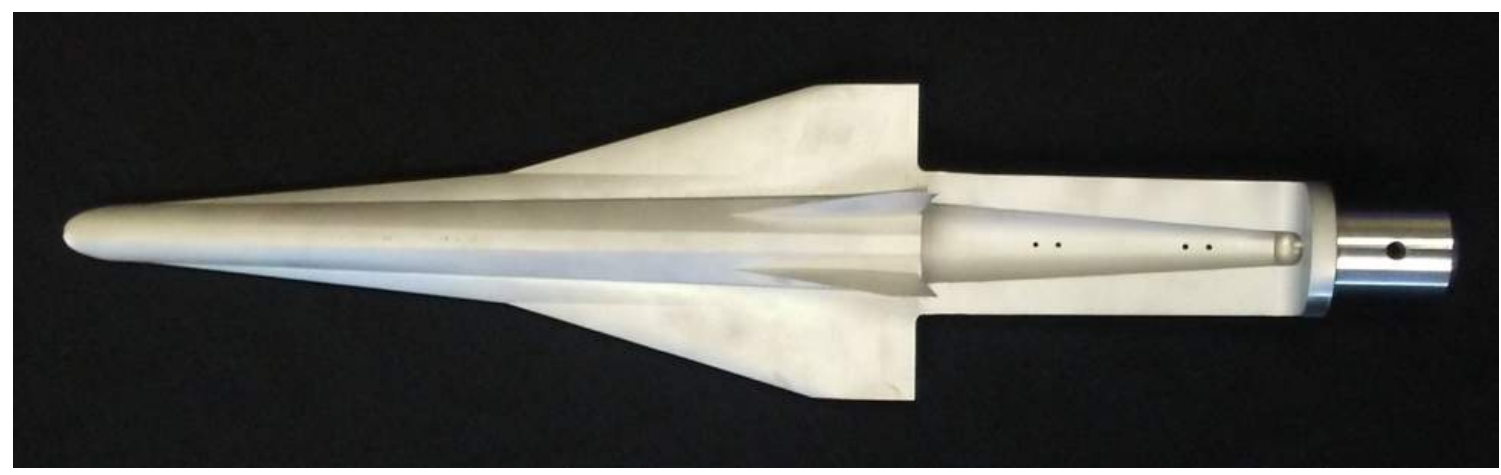

Figure 2.13: Unpainted GHV wind tunnel model. 


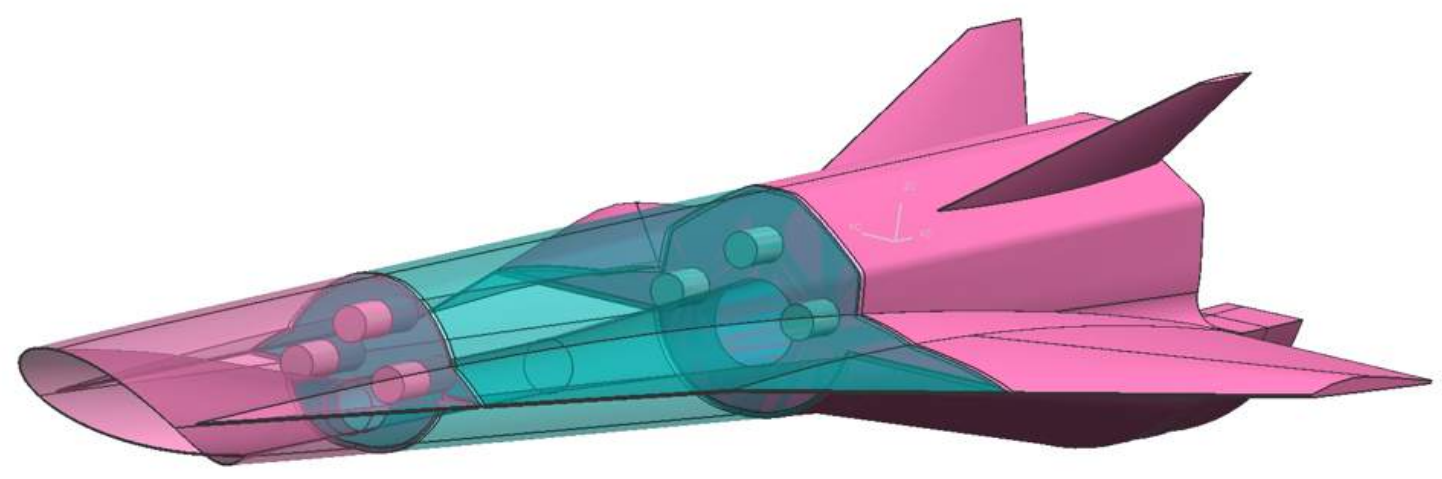

Figure 2.14: GHV rendered CAD model in three pieces.

This weld and location of the instrumentation on the sting are shown in Figure 2.15 where the two pressure tabs and two thermocouples can also be seen. Again this was not the ideal location for the instrumentation on this model, due to the fact that the flow in the wake of the model will most likely be "dirty" and will not yield great results. However, this placement was chosen out of necessity due to the many extremely thin sections of the model and it was determined that instrumentation in any other part of the model was not really feasible. 


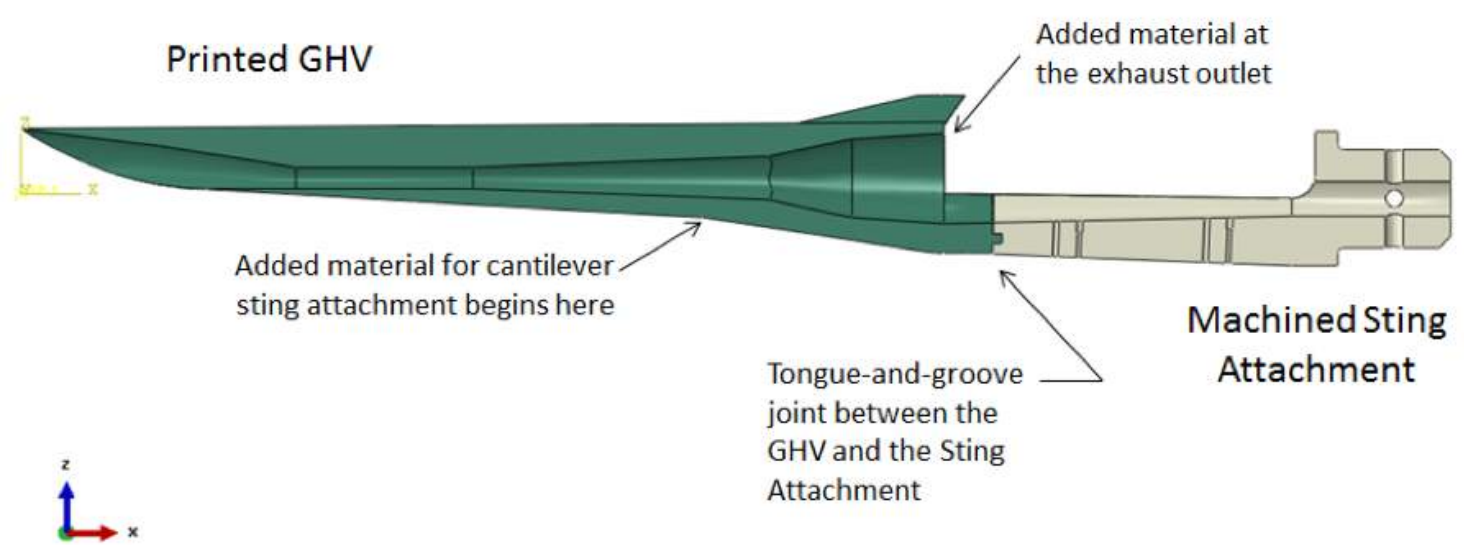

Figure 2.15: GHV rendered CAD model side view with sting.

\subsection{Experimental Testing}

With the Mach 6 wind tunnel being unused for the past 20 years, a resurgence in the need for accurate high speed testing facilities has resulted in the wind tunnel becoming operational once again. A point of interest in hypersonic testing as previous noted, is the surface temperature distribution which can be obtained by using Temperature Sensitive Paint (TSP). The paint is applied evenly as a thin layer on the surface and a Charge Coupled Device (CCD) array can capture the instantaneous change in color of the paint which can then be correlated to local temperatures. The TSP is calibrated by Innovative Scientific Solutions Incorporated (ISSI) for certain temperature ranges with a second-order accurate curve fit applied to the calibration data based on luminosity. The temperature value is a function of the ratio of wind-off to wind-on luminosity.

The TSP paint was calibrated by the supplier ISSI, and was slightly modified by the experimental testing staff to determine if it was possible to run at slightly higher temperatures than initially indicated on the calibration curve. As shown in Figure 2.16, a curve fit was used to extrapolate the 
corresponding temperature based off of the paint luminosity. Both the temperature and luminosity are based off the wind off references values in the tunnel.

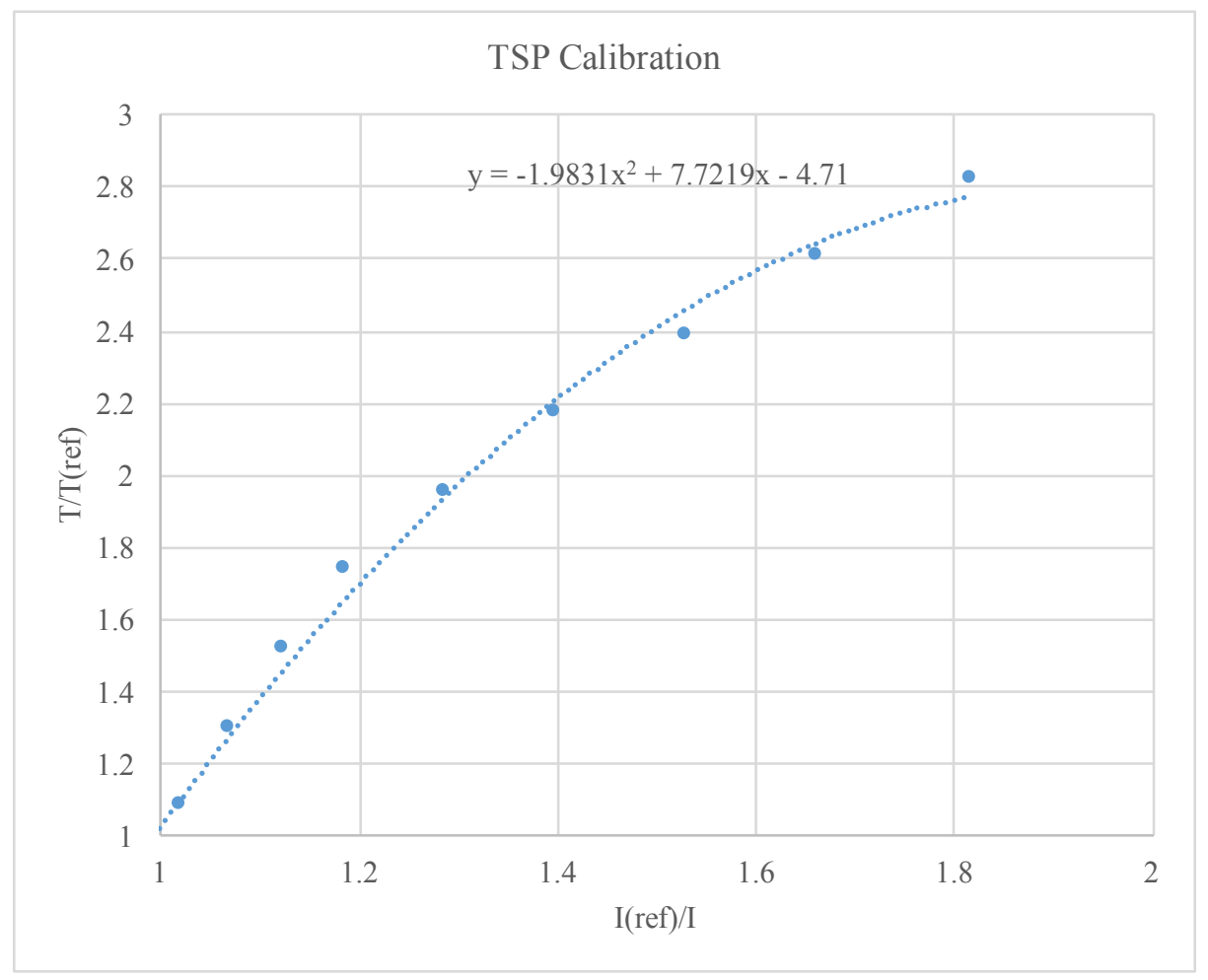

Figure 2.16: TSP calibration curve.

\subsubsection{HIFiRE-1 Results}

The test matrix shown in Table 2.2 was used in testing the HIFiRE-1 geometry in the Mach 6 wind tunnel. This matrix was first completed without TSP to have clean readings of the pressure taps and thermocouples without the interference of the paint in the instrumentation holes and then repeated with the use of TSP while continuing to take readings from the instrumentation. Note the chosen stagnation pressure and temperature vales are spanning each end of the spectrum outlined in the Mach 6 Wind Tunnel test section conditions (Table 2.1). This was done to evaluate the paints 
ability to adhere to the models under the various conditions of the Mach 6 wind tunnel. The behavior of the paint adhesion for this model will guide the test planning of future thermal tests using TSP in the Mach 6 tunnel. The painted HIFiRE-1 wind tunnel model mounted in the Mach 6 tunnel can be seen in Figure 2.17

Table 2.2: Mach 6 Wind Tunnel Testing Matrix for HIFiRE-1 Geometry.

\begin{tabular}{c|c|c|c} 
Total Pressure $\left(P_{0}\right)$ & Total Temperature $\left(T_{0}\right)$ & Orientation & AOA [Deg] \\
\hline \hline 700 & 900 & Notch Starboard & $-10,-5,0,5,10$ \\
\hline 700 & 1000 & Notch Starboard & $-10,-5,0,5,10$ \\
\hline 900 & 900 & Notch Starboard & $-10,-5,0,5,10$ \\
\hline 900 & 1000 & Notch Starboard & $-10,-5,0,5,10$
\end{tabular}

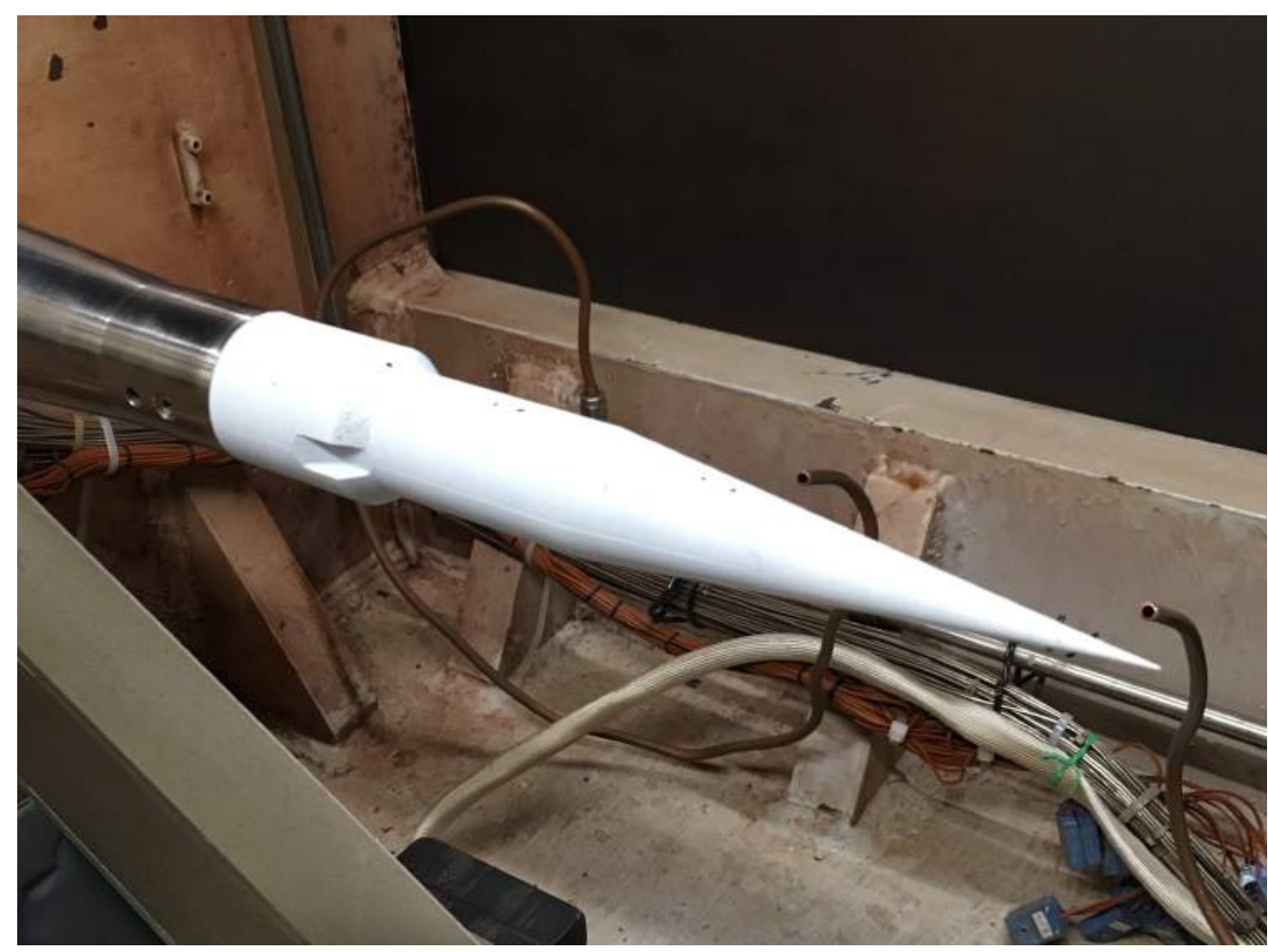

Figure 2.17: HIFiRE-1 mounted wind tunnel model. 
A sample of the results from the experimental testing of the HIFiRE-1 model can be seen in Figures 2.18 and 2.19. In Figure 2.18 the pressure coefficient, read out from the pressure taps, is plotted when $P_{0}=700 \mathrm{psi}$ and $T_{0}=900 R$ for $A O A=-10,-5,0,5,10$. Under the same conditions, Figure 2.19 shows the thermocouple readings for the local surface temperature. The pressure coefficient is based on the test section's dynamic pressure, which was calculated using isentropic conditions. The fact that the models never reach an equilibrium temperature is the reason the thermocouple readings never reach a constant value. The period at which the model was injected into the flow is indicated by the red line equaling one. The model angle of attack is also indicated by the blue line in the below figures.

As expected the highest value for $C_{p}$ is experienced at $P_{T}=3$ due to its placement at the high inclination region on the model. Even at positive angles of attack, when the instrumentation is leeward of the flow, a relatively high value for $C_{p}$ is recorded for $P_{T}=3$ due to the re-attachment of the flow at this point on the model. The value for $C_{p}$ decreases at pressure tap 3 as the angle between the flow and the normal off the body increased, which makes sense as this point becomes less of a stagnation region when the body increases its relative angle of attack with the flow.

Results from the thermocouples, shown in Figure 2.19, show that the model never reaches its equilibrium or recovery temperature indicated by the steady increase in temperature during the test period. This proved to be one of the areas that caused difficulties in comparing the experimental and numerical results since this temperature at a specific snap shot in time was dependent on multiple factors, like conduction through the model, that were not modeled in the numerical analysis. Experimental results for other test conditions are shown in Appendix A. 

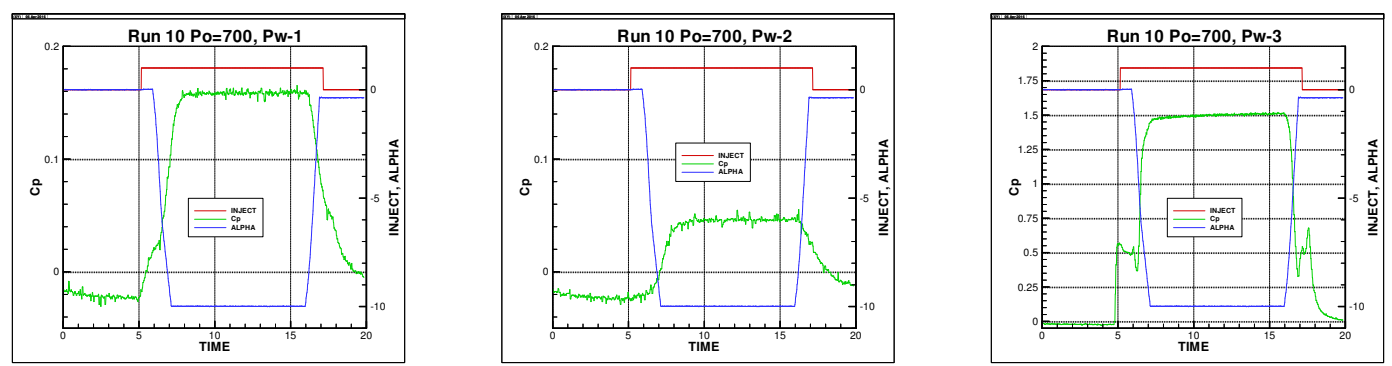

$P_{T}=1, A O A=-10$
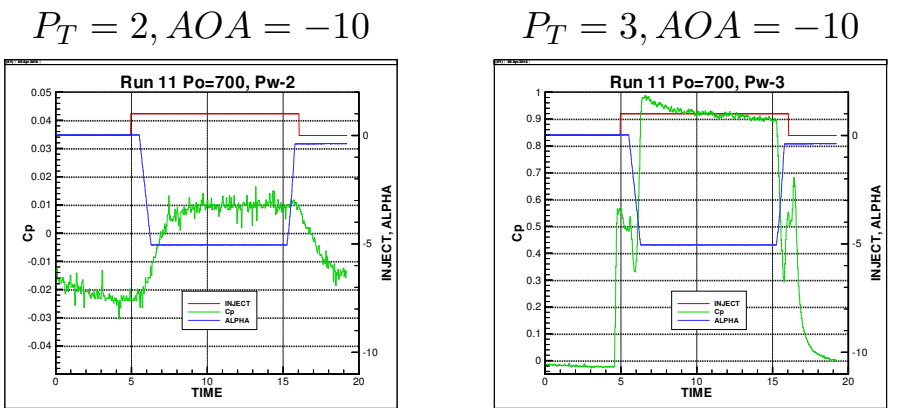

$$
P_{T}=1, A O A=-5
$$

$$
P_{T}=2, A O A=-5
$$

$$
P_{T}=3, A O A=-5
$$
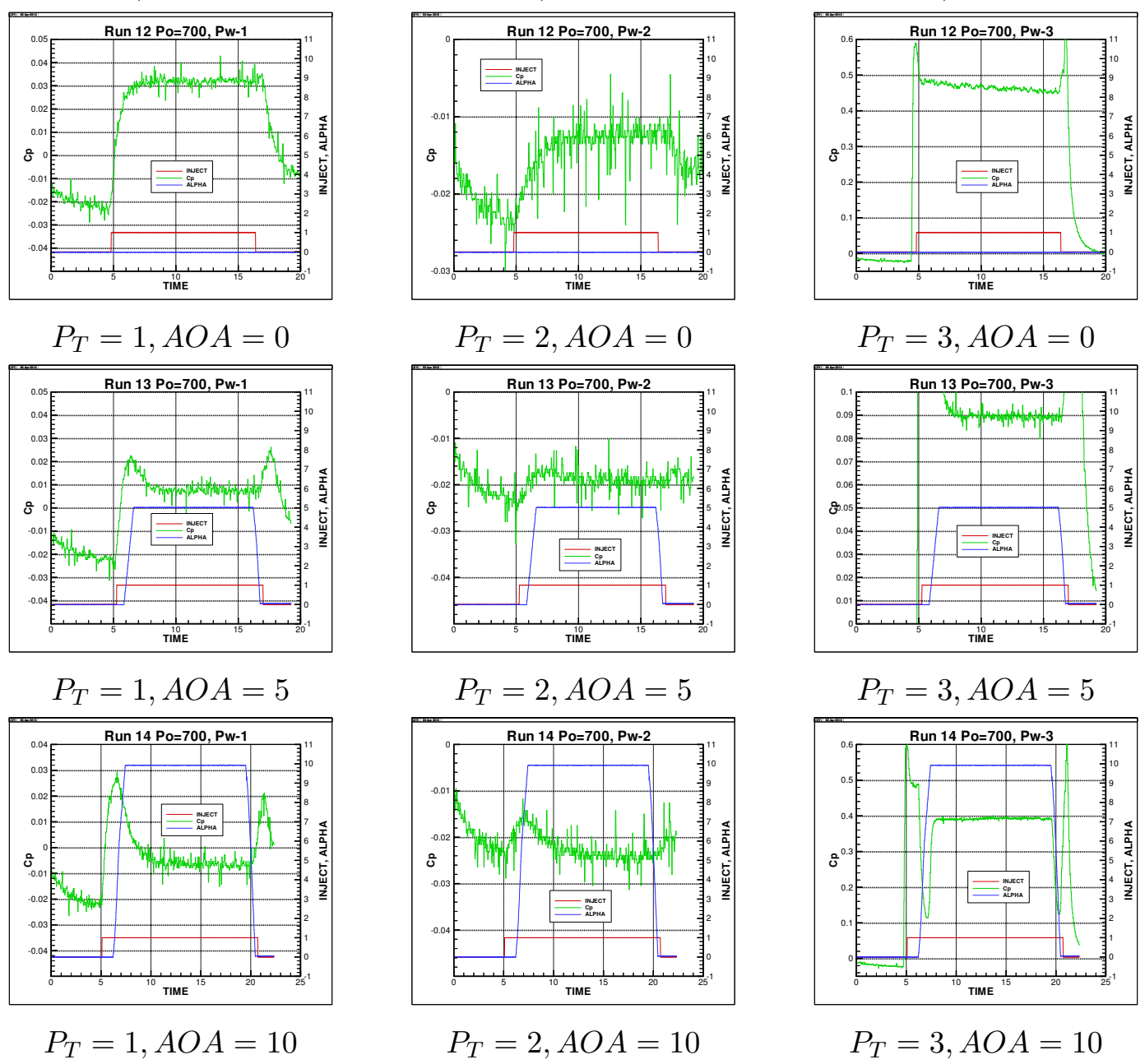

Figure 2.18: HIFiRE-1 Mach 6 Wind Tunnel results for $C_{p}$ at various AOA for $P_{0}=700$ psi and $T_{0}=900 R$. 

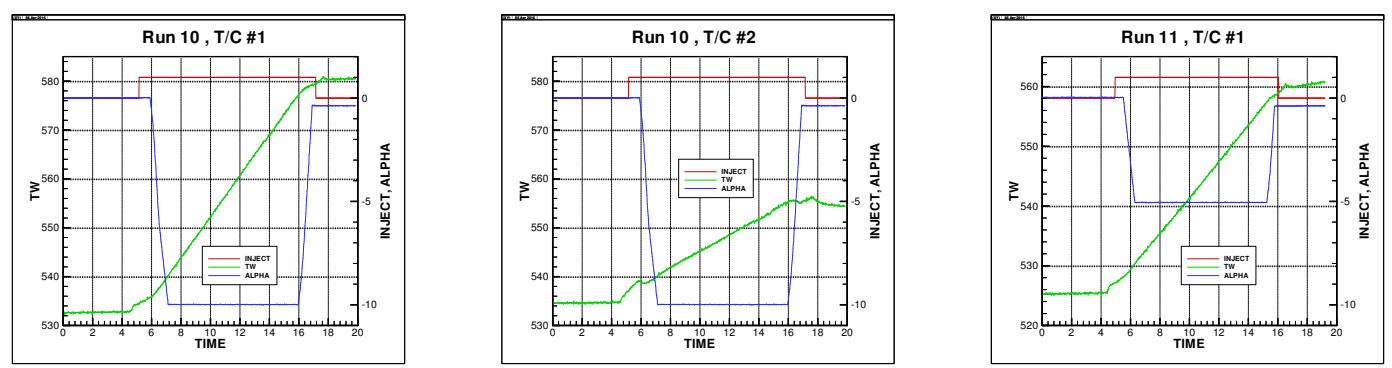

$T_{C}=1, A O A=-10$

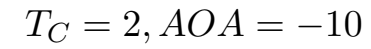

$T_{C}=1, A O A=-5$
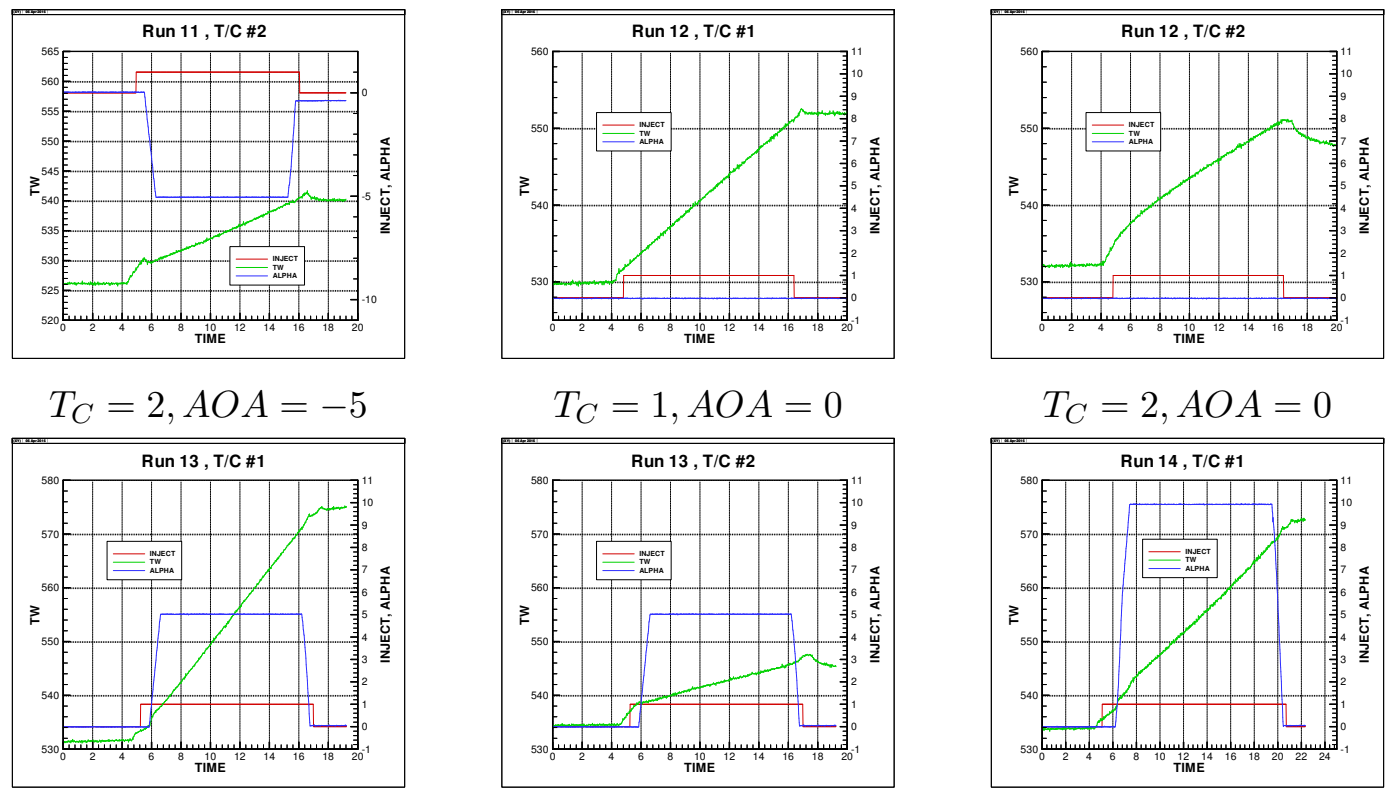

$$
T_{C}=1, A O A=0
$$

$$
T_{C}=2, A O A=0
$$
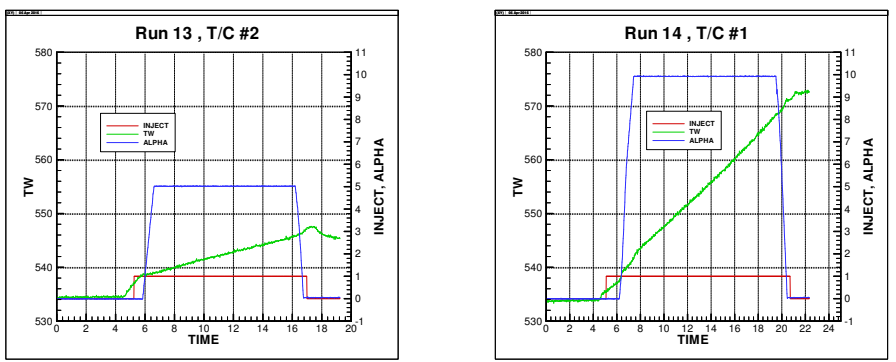

$$
T_{C}=1, A O A=5
$$

\section{$T_{C}=2, A O A=5$}

$T_{C}=1, A O A=10$

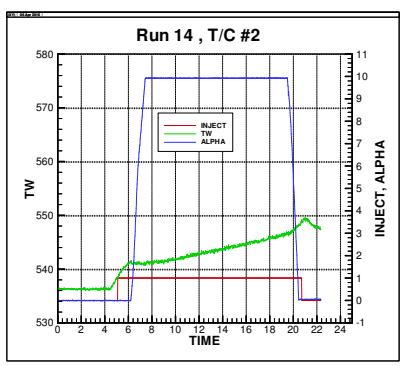

$$
T_{C}=2, A O A=10
$$

Figure 2.19: HIFiRE-1 Mach 6 Wind Tunnel results for surface temperature at various AOA for $P_{0}=700$ psi and $T_{0}=900 R$.

The TSP contour results for the HIFiRE- 1 for $P_{0}=700$ psi and $T_{0}=900 R$ are shown in

Figure 2.20. As expected, the highest surface temperature is located at the nose tip and on the 
windward side high inclination region. This image was taken after the model was on centerline for 3 seconds. The high temperature pin size spots on the windward side are due to the epoxy filling in the dowel holes, which has a higher thermal conductivity than the stainless steel. Finally, the uneven temperature distribution on the leeward side of the high inclination region at $A O A=10$ is most likely due to flow reattachment from the windward side of the model experienced at high angles of attack.

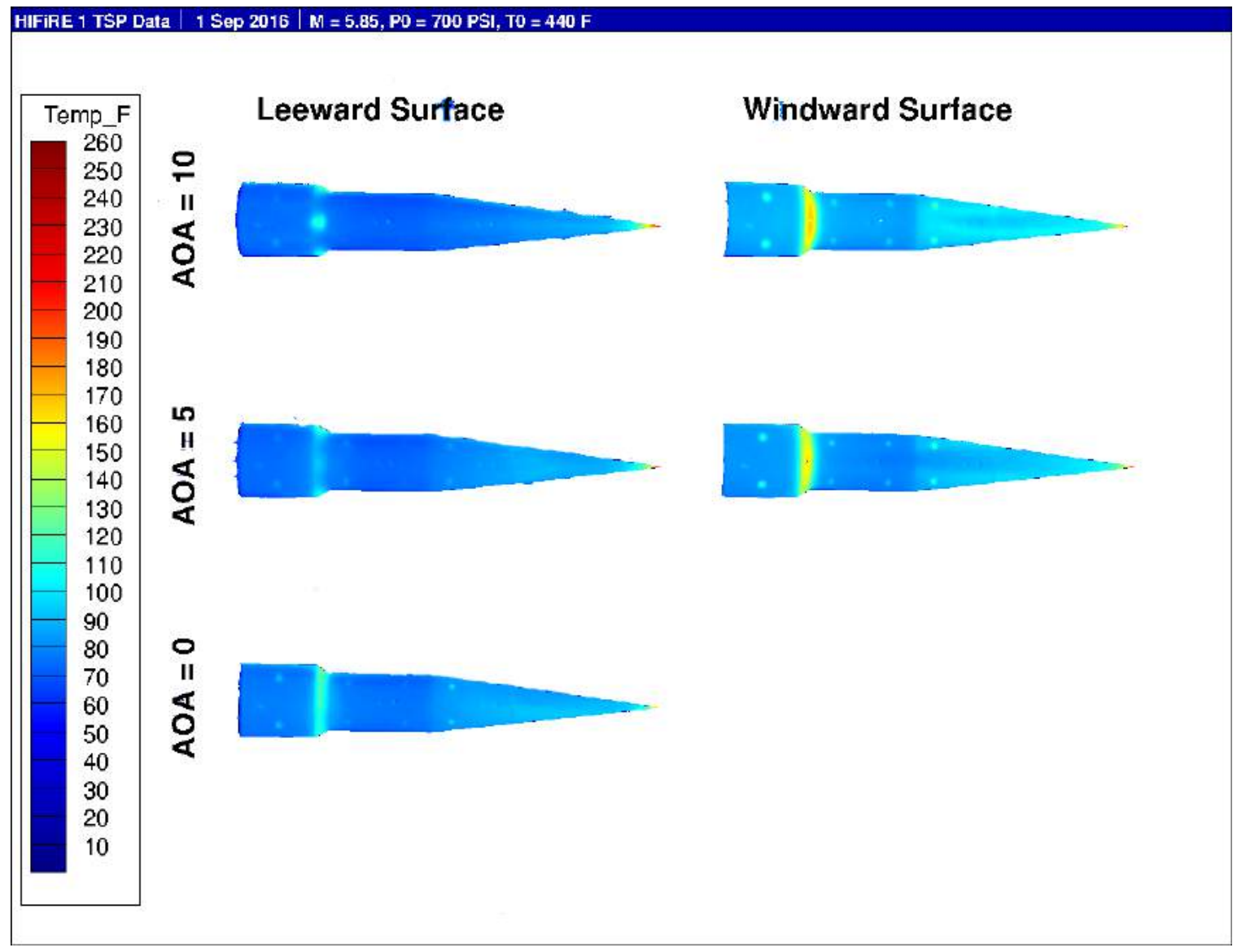

Figure 2.20: HIFiRE-1 TSP temperature contours for $P_{0}=700 \mathrm{psi}$ and $T_{0}=900 \mathrm{R}$. 


\subsubsection{RFSG Results}

For the RFSG model, findings from the testing of the HIFiRE-1 model were used to influence the test set up of the RFSG. First the need to run tests without the paint to obtain clean readings with the instrumentation was found unnecessary and therefore the instrumentation readings were taken with the pain applied. Also, the RFSG was tested at the wind tunnel conditions that resulted in the best paint adhesion and response. Therefore the low pressure and high temperature wind tunnel conditions were chosen for both the RFSG and GHV, shown in Tables 2.3 and 2.4, respectively. Due to the RFSG and GHV being asymmetric, the models were also mounted inverted to capture the paint response for both the windward and leeward sides for each angle of attack.

The painted RFSG wind tunnel model is shown in Figure 2.21 where extreme paint detachment was experienced around the winglets and nose region. However, due to the overhead mounting of the camera, these regions were never captured anyway except in the extreme $A O A=10$ degree case.

The results from experimental testing of the RFSG model can be seen in Figures 2.22-2.26. In Figures $2.22-2.25$ the pressure coefficients, given by the eight pressure taps, are plotted when $P_{0}=$ $700 \mathrm{psi}$ and $T_{0}=1000 R$ for $A O A=-10,-5,0,5,10$. Under the same conditions, Figure 2.26 shows the thermocouple reading for surface temperature.

Table 2.3: Mach 6 Wind Tunnel Testing Matrix for RFSG Geometry.

\begin{tabular}{c|c|c|c} 
Total Pressure $\left(P_{0}\right)$ & Total Temperature $\left(T_{0}\right)$ & Orientation & AOA [Deg] \\
\hline \hline 700 & 1000 & Normal & $-10,-5,0,5,10$ \\
\hline 700 & 1000 & Inverted & $-10,-5,0,5,10$
\end{tabular}




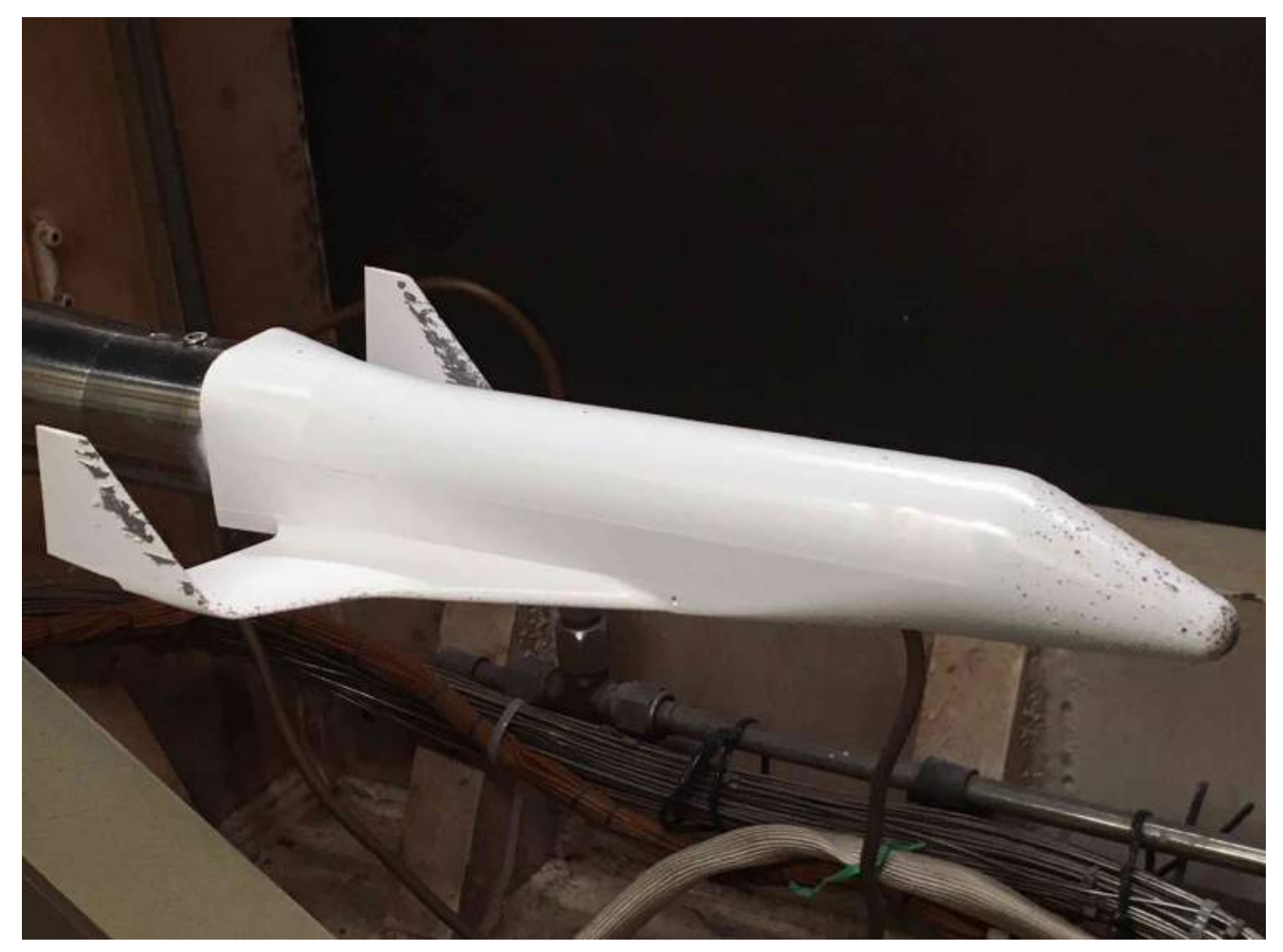

Figure 2.21: RFSG mounted wind tunnel model. 

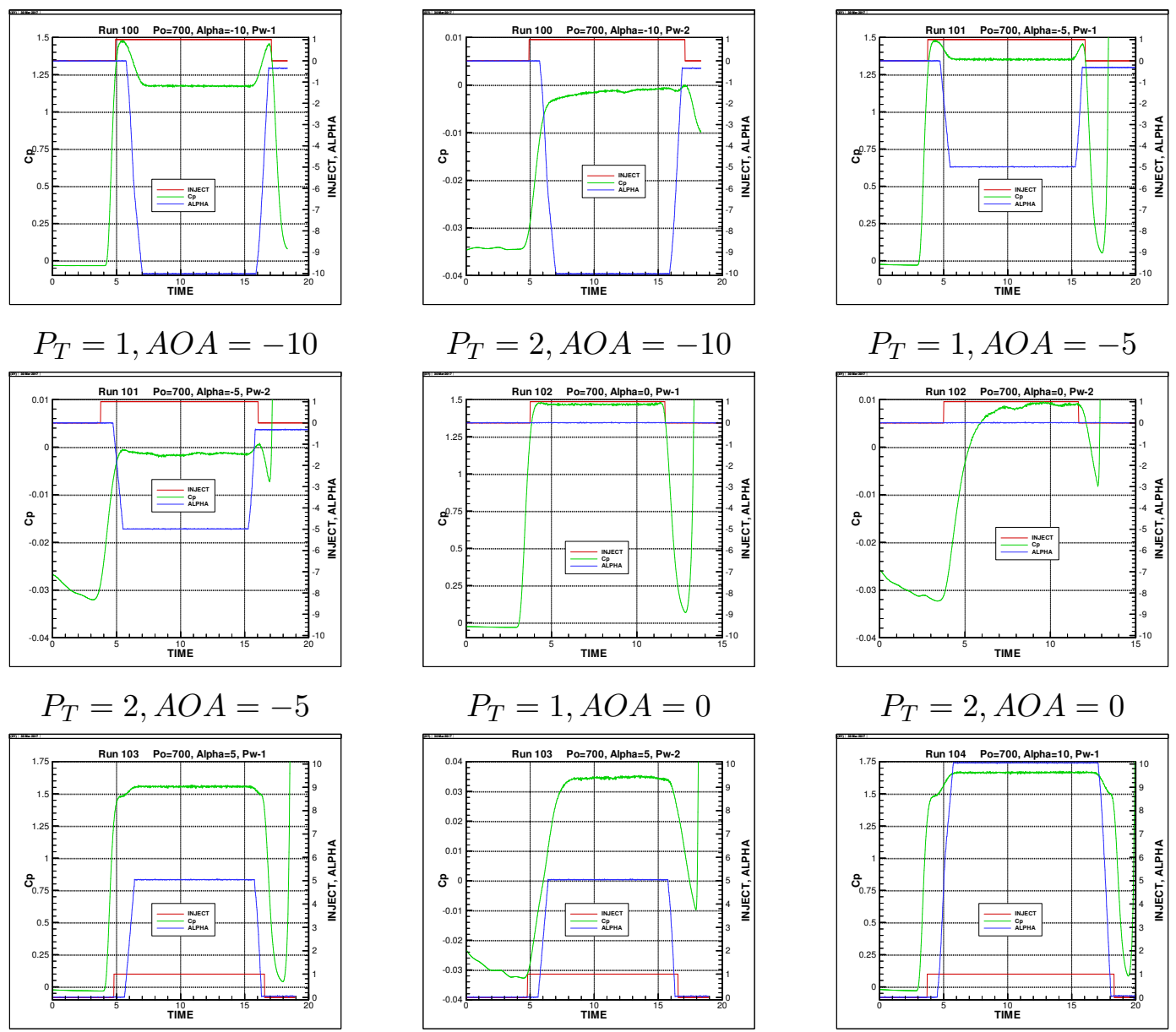

$$
P_{T}=1, A O A=5
$$

$$
P_{T}=2, A O A=5
$$

$$
P_{T}=1, A O A=10
$$

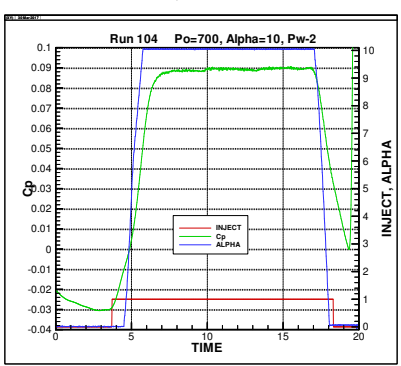

$$
P_{T}=2, A O A=10
$$

Figure 2.22: RFSG Mach 6 Wind Tunnel results for $C_{p}$ at various AOA for $P_{0}=700$ psi and $T_{0}=1000 R$ at $P_{T}=1,2$. 

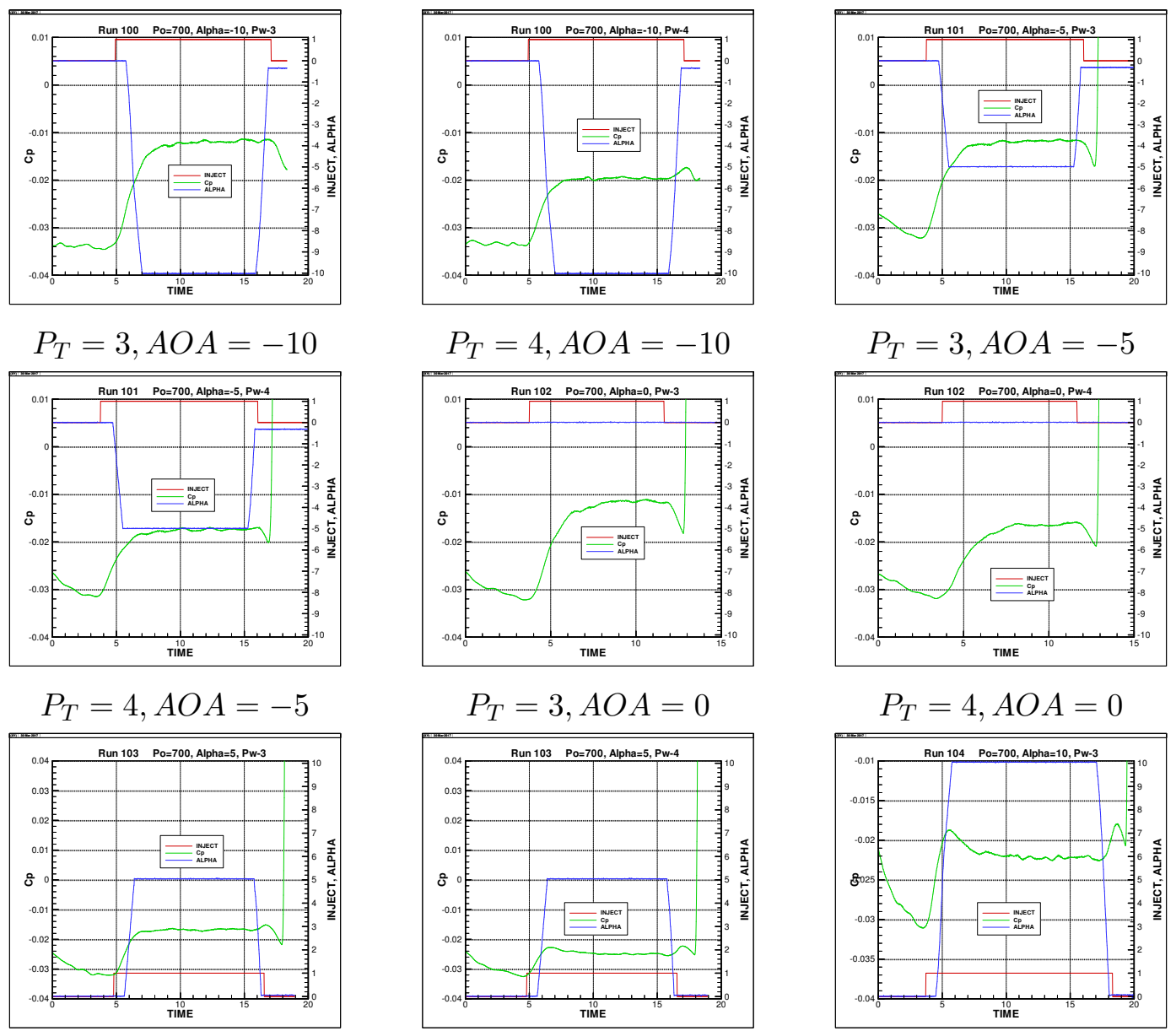

$$
P_{T}=3, A O A=5
$$

$$
P_{T}=4, A O A=5
$$

$$
P_{T}=3, A O A=10
$$

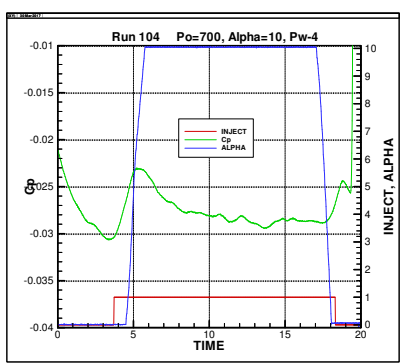

$$
P_{T}=4, A O A=10
$$

Figure 2.23: RFSG Mach 6 Wind Tunnel results for $C_{p}$ at various AOA for $P_{0}=700$ psi and $T_{0}=1000 R$ at $P_{T}=3,4$. 

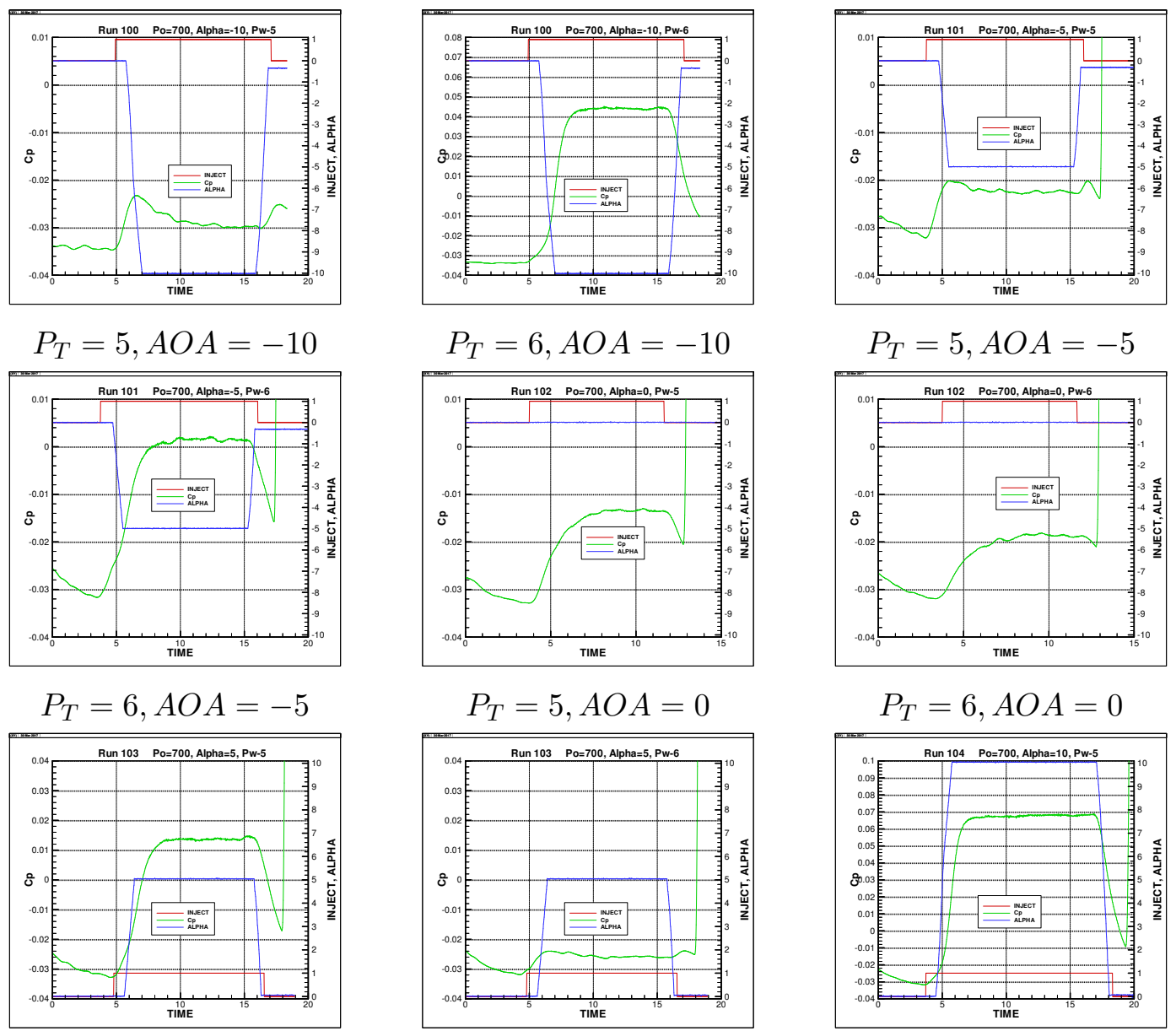

$$
P_{T}=5, A O A=5
$$

$$
P_{T}=6, A O A=5
$$

$$
P_{T}=5, A O A=10
$$

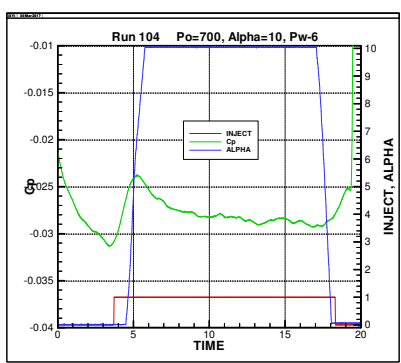

$$
P_{T}=6, A O A=10
$$

Figure 2.24: RFSG Mach 6 Wind Tunnel results for $C_{p}$ at various AOA for $P_{0}=700$ psi and $T_{0}=1000 R$ at $P_{T}=5,6$. 

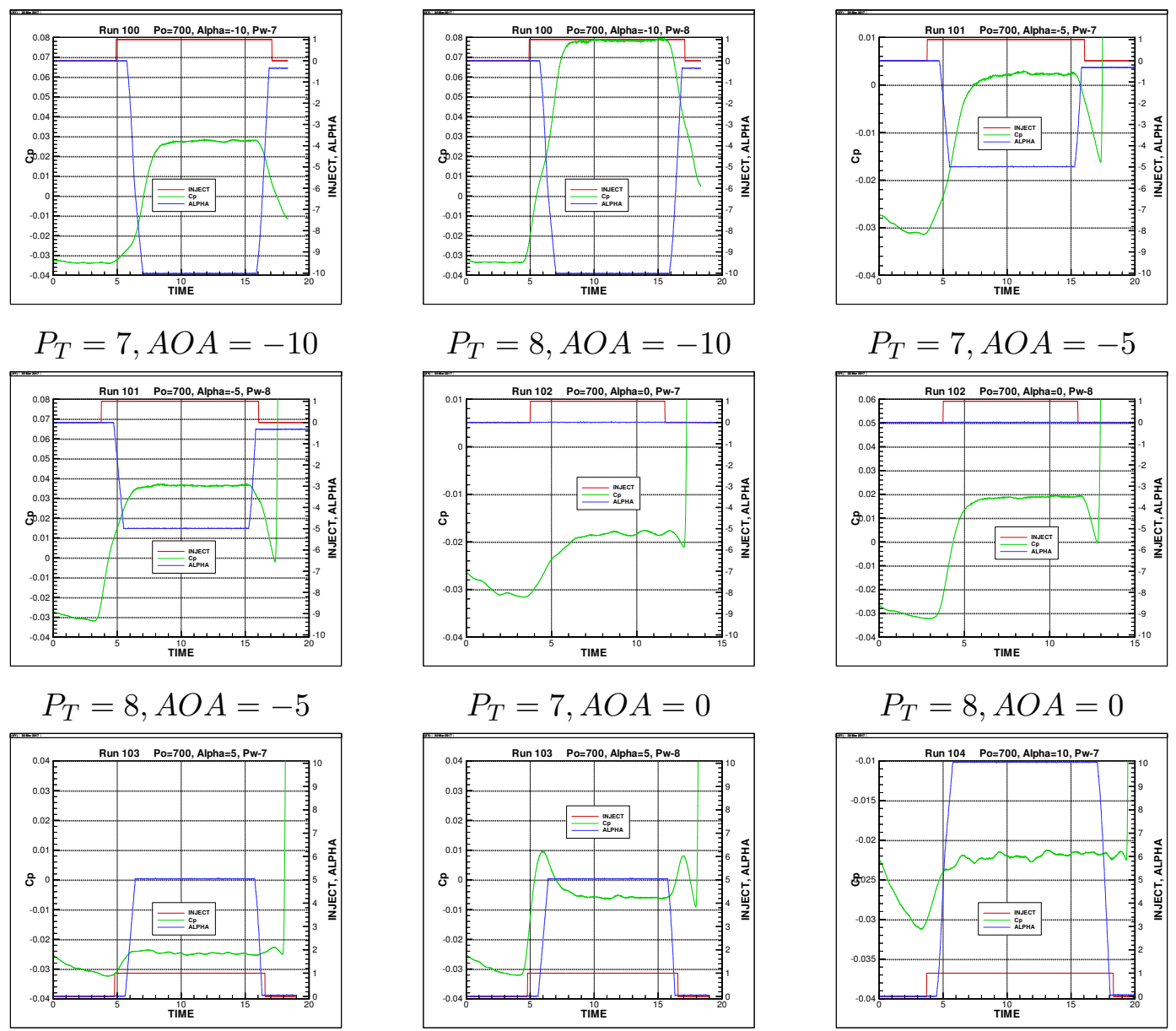

$$
P_{T}=7, A O A=5
$$

$$
P_{T}=8, A O A=5
$$

$$
P_{T}=7, A O A=10
$$

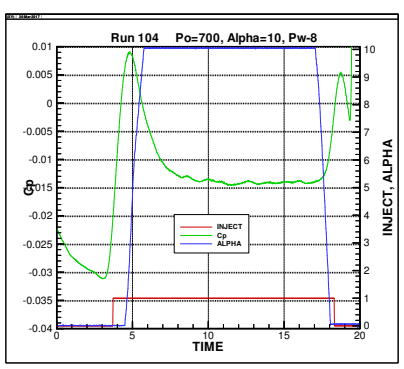

$$
P_{T}=8, A O A=10
$$

Figure 2.25: RFSG Mach 6 Wind Tunnel results for $C_{p}$ at various AOA for $P_{0}=700$ psi and $T_{0}=1000 R$ at $P_{T}=7,8$. 


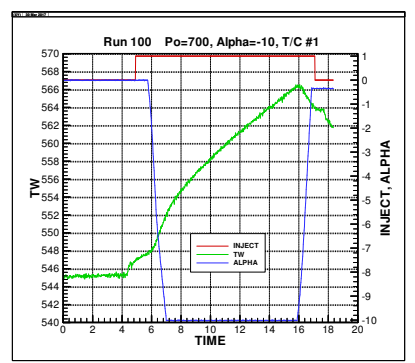

$T_{C}=1, A O A=-10$

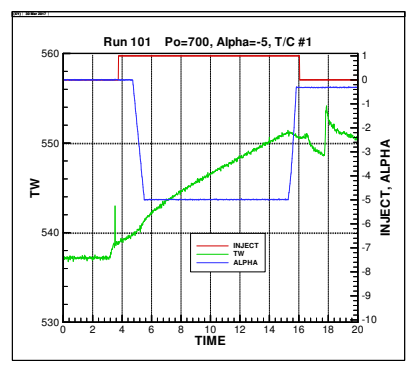

$$
T_{C}=1, A O A=-5
$$

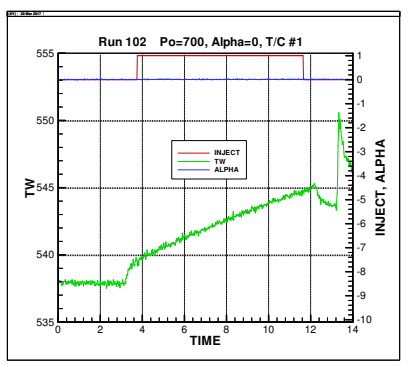

$$
T_{C}=1, A O A=0
$$

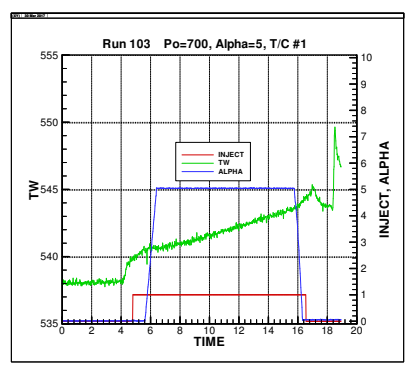

$$
T_{C}=1, A O A=5
$$

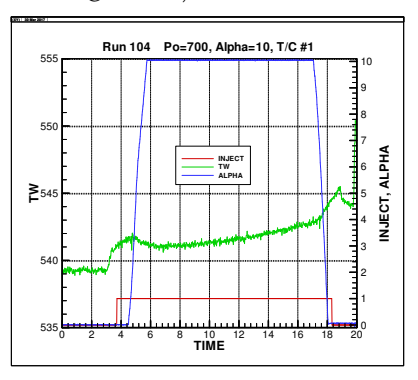

$$
T_{C}=1, A O A=10
$$

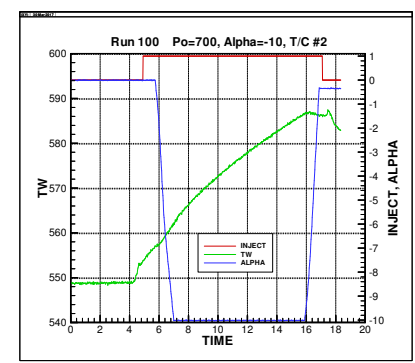

$T_{C}=2, A O A=-10$

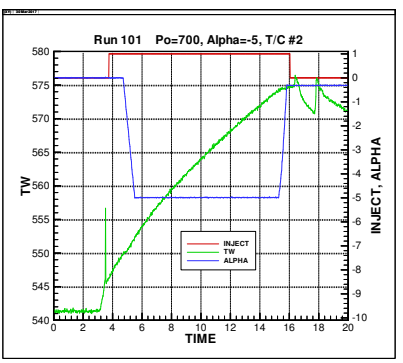

$$
T_{C}=2, A O A=-5
$$

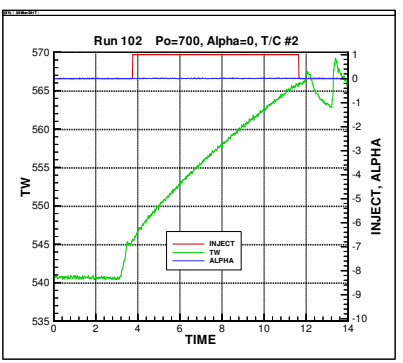

$$
T_{C}=2, A O A=0
$$

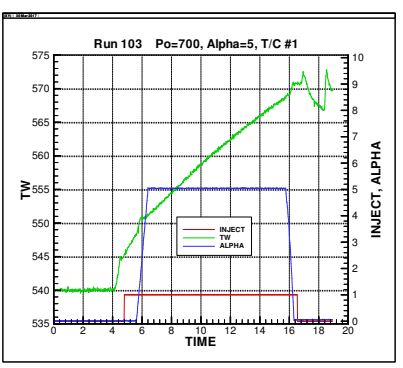

$$
T_{C}=2, A O A=5
$$

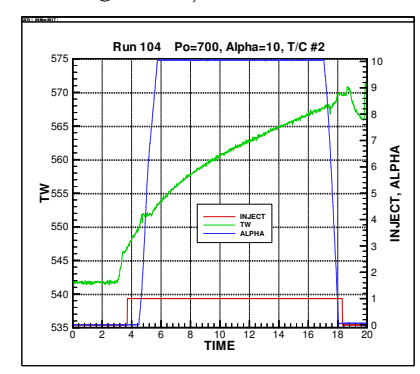

$T_{C}=2, A O A=10$

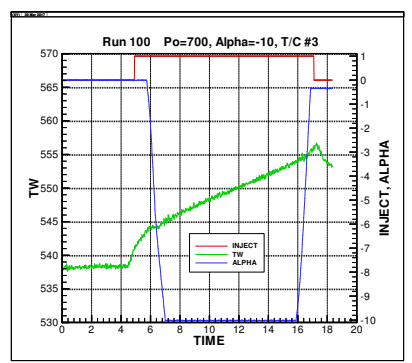

$T_{C}=3, A O A=-10$

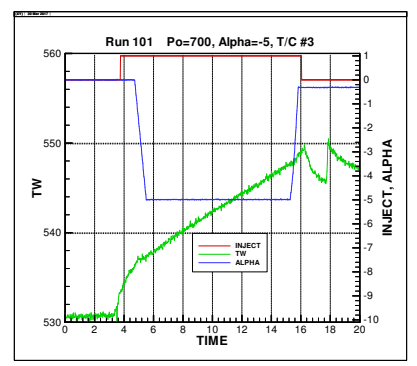

$$
T_{C}=3, A O A=-5
$$
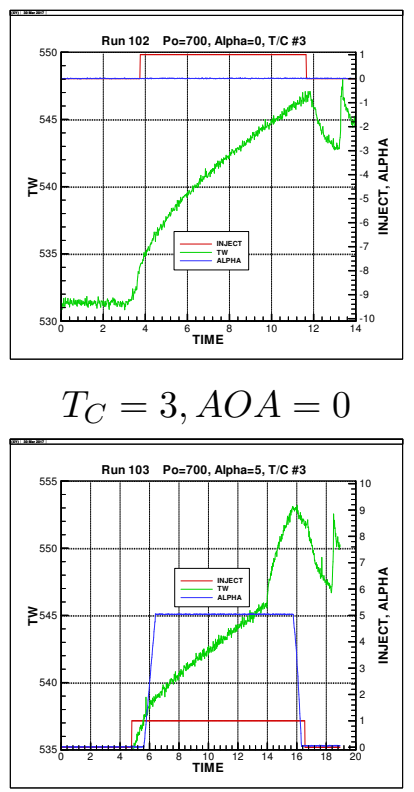

$$
T_{C}=3, A O A=5
$$

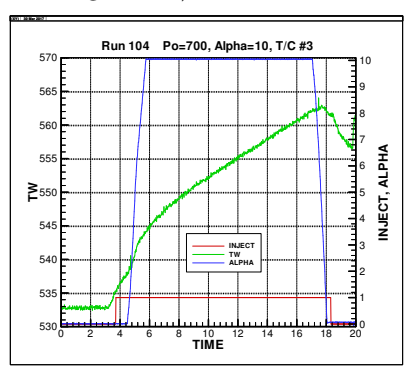

$$
T_{C}=3, A O A=10
$$

Figure 2.26: RFSG Mach 6 Wind Tunnel results for surface temperature at various AOA for $P_{0}=$ $700 \mathrm{psi}$ and $T_{0}=1000 R$ at $T_{c}=1,2,3$. 
As seen in Figure 2.27, the temperature contour for the RFSG shows higher temperatures at the stagnation points around the leading edge of the wings and nose compared to the HIFiRE-1 model. One can note the circular hole section on the lower surface of the RFSG where once again epoxy was added to fill in the screw holes connecting the two pieces of the RFSG.

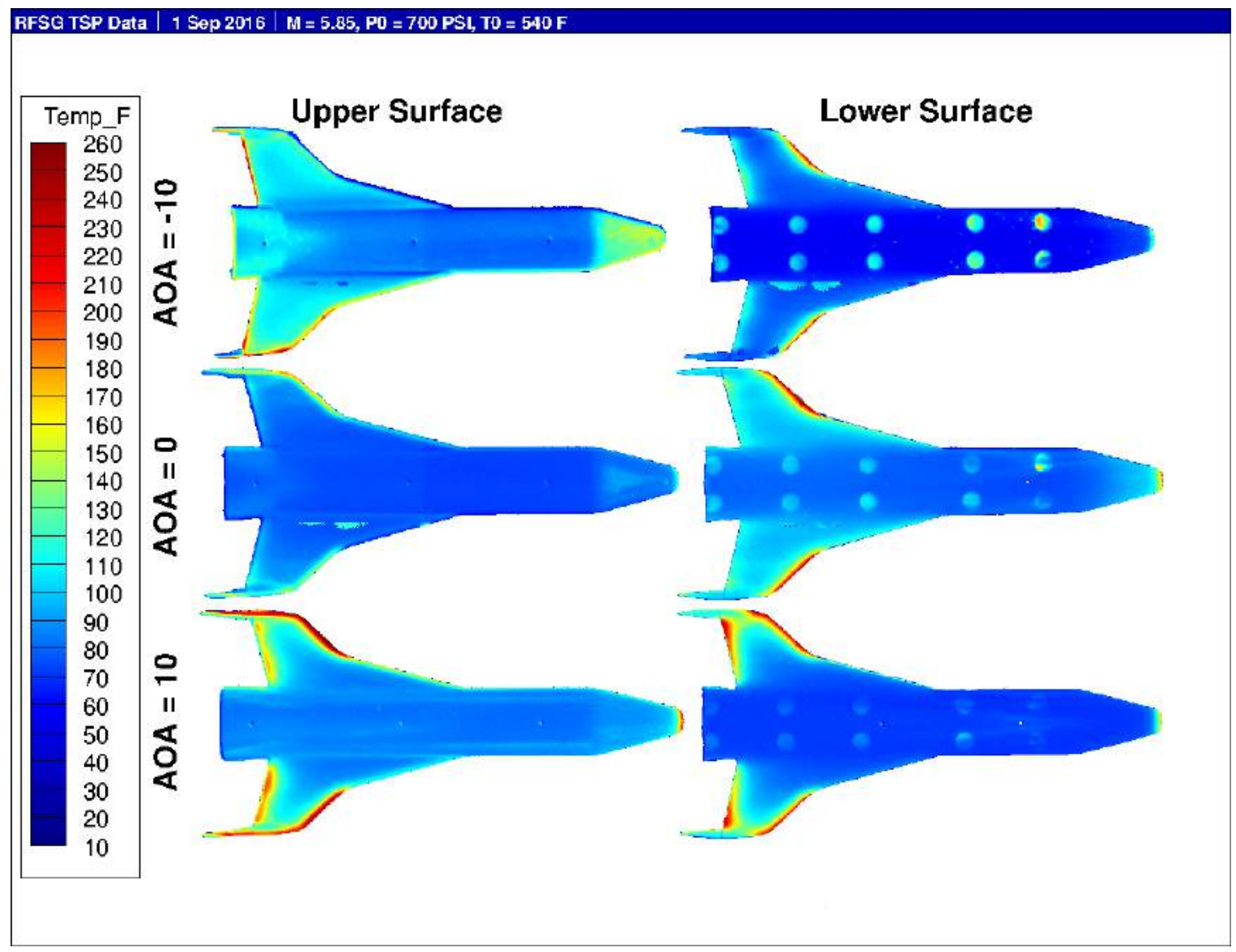

Figure 2.27: RFSG TSP Temperature Contour.

\subsubsection{GHV Results}

The experimental test matrix for the GHV model is given in Table 2.4 where again the low pressure $\left(P_{0}=700 \mathrm{psi}\right)$ and the high temperature $\left(T_{0}=1000 R\right)$ wind tunnel conditions were 
evaluated. The $C_{p}$ and temperature plots are not shown here due to their unimportant locations on the sting of the model.

The significance of the testing of the GHV model was to obtain the temperature contours using the TSP which is shown in Figure 2.29 where a high temperature was seen at the inlet and along the leading edge of the wing and vertical fins. Due to high conduction through the model, especially through the very thin wings, determining where the heat was coming from was very difficult once the model was in the flow for longer than a few seconds. Even with this conduction through the model, the extreme temperature seen at the models inlet was expected as this acted as a stagnation point as the flow entered the internal flow-path. An example of the conduction through the thin model is shown by the increased temperature aft of the inlet at zero degrees AOA, which proceeded through the inlet stagnation point into the model. This is a case where the surface temperature measured by the TSP was not a direct result of the flows interaction with this point on the OML and instead was a result of the conduction through the models surface from alternate directions. 


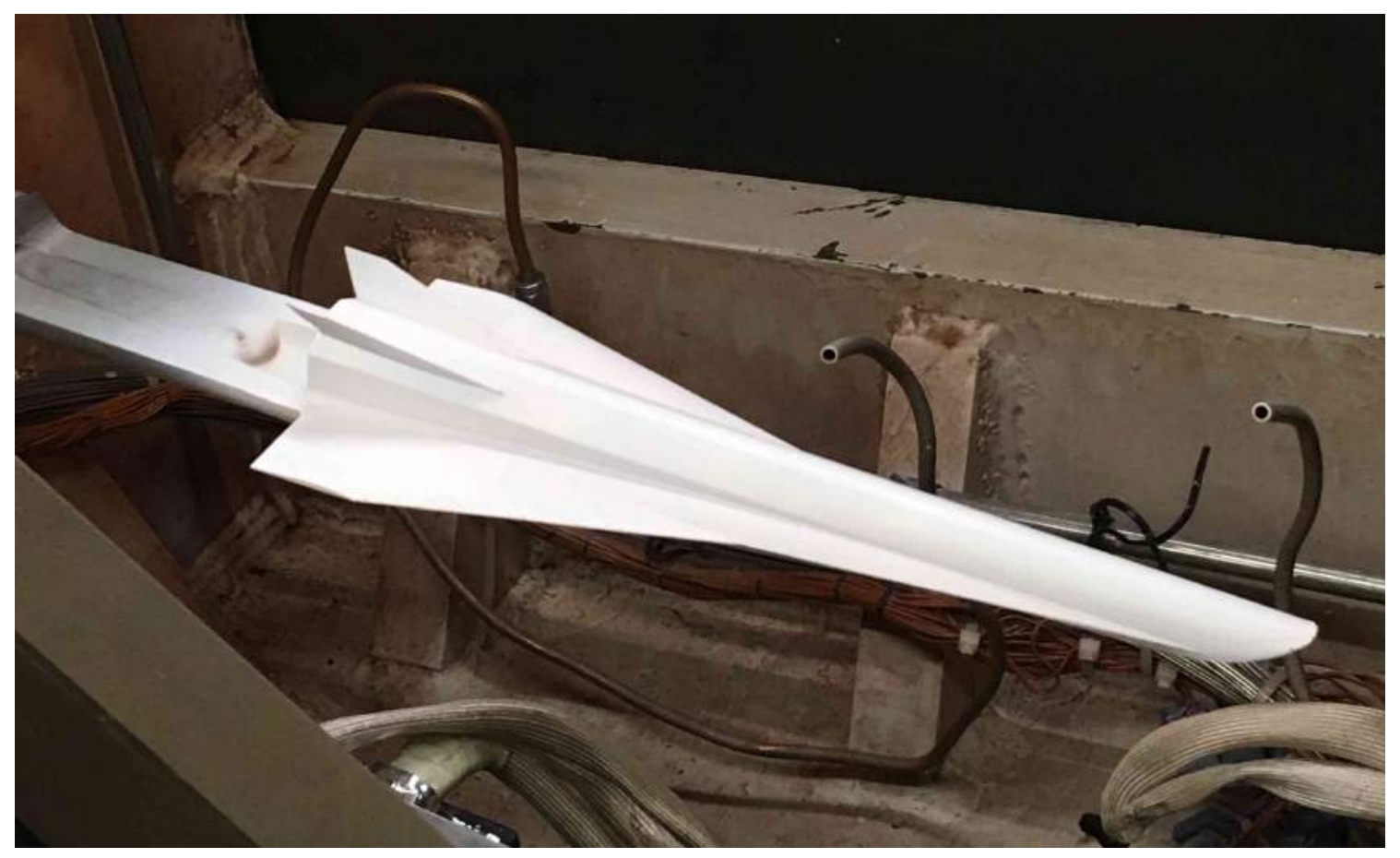

Figure 2.28: GHV mounted wind tunnel model.

Table 2.4: Mach 6 Wind Tunnel Testing Matrix for GHV Geometry.

\begin{tabular}{c|c|c|c} 
Total Pressure $\left(P_{0}\right)$ & Total Temperature $\left(T_{0}\right)$ & Orientation & AOA [Deg] \\
\hline \hline 700 & 1000 & Normal & $-10,-8,-5,0,5,8,10$ \\
\hline 700 & 1000 & Inverted & $-10,-8,-5,0,5,8,10$
\end{tabular}




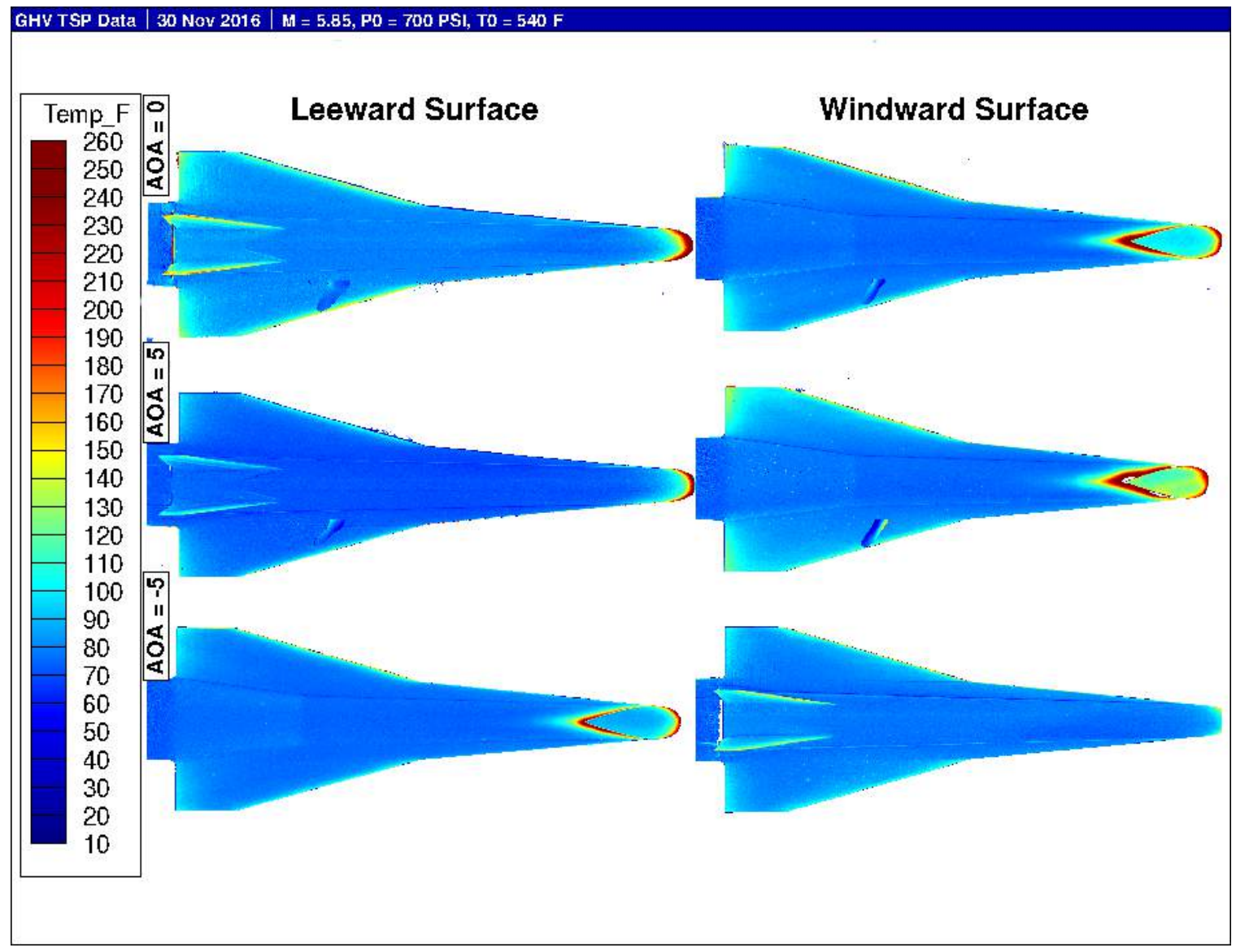

Figure 2.29: GHV TSP temperature contour for $P_{0}=700 \mathrm{psi}$ and $T_{0}=1000 R$. 


\section{CHAPTER III}

\section{INVISCID NUMERICAL ANALYSIS}

To gain confidence in the experimental results obtained from the Mach 6 wind tunnel, a panel code with viscous approximations namely the Configuration Based Aerodynamics (CBAero) [15] tool set and Euler solver Cart3D [16, 17] were applied to all three models. Both analysis tools were developed by the National Aeronautics and Space Administration (NASA). Prior to reporting results from any of these codes grid convergence studies were conducted on computational meshes built specifically for each of the analysis tools. Final meshing strategies were chosen from the grid convergence studies that incurred acceptable discretization errors in the integrated quantities of interest (lift and drag coefficients, $C_{L}$ and $C_{D}$ ) derived from the flow solutions. Once appropriate meshes were chosen for each tool, attempts were made to obtain surface wall temperatures as well as pressure distributions for each geometry. Computational setup and results for all three models using the low-fidelity CBAero code are explained in Section 3.1 and the medium-fidelity results using Cart3d are shown in Section 3.2

\subsection{CBAero Panel Code Analysis}

The CBAero software package is an engineering-level inviscid flow solver that incorporates various panel methods in order to evaluate the aerodynamic and aero-thermodynamic response of arbitrary vehicle configurations at supersonic and hypersonic speeds. A beneficial attribute of the code is its use of unstructured surface triangulations to define input geometry [15]. The use of 
the term panel denotes that an integrated quantity obtained from one surface cell of a geometry is independent of any quantity obtained from any other cell. In other words, there is no flow interaction or communication from one cell to the next. To provide an estimate of the surface velocity vectors at hypersonic speeds, Equation (3.1) can be used.

$$
\vec{V}_{\text {surface }}=\hat{n} \times \vec{V}_{\infty} \times \hat{n}
$$

Various inviscid panel methods are available for analysis such as Modified Newtonian, Tangent Cone, and Tangent Wedge [7]. The methods determine approximated values of pressure coefficients acting normally on each cell of the geometry. A different method may be assigned to different sets of cells or components that, when subjected to a free stream velocity, more closely align with the assumptions of an available panel method. Once the inviscid solution is obtained from a chosen panel method strategy, CBAero provides its own viscous approximation to estimate surface skin friction. From the skin friction estimate, heating rates and temperatures at the body wall may be determined [18].

For this effort, the Modified Newtonian method is used for the inviscid approximation of surface pressures on the HIFiRE-1, RFSG and GHV geometries. The flow conditions for the CBAero analysis, corresponding to the Mach 6 wind tunnel conditions, are shown in Table 3.1 . The grid convergence studies and inviscid analysis results are shown in Sections 3.1.1 3.1.3 for each geometry. 
Table 3.1: CBAero Flow Analysis Conditions

\begin{tabular}{c|c|c|c|c} 
& Case 1 & Case 2 & Case 3 & Case 4 \\
\hline \hline$P_{0}[\mathrm{psi}]$ & 700 & 700 & 2000 & 2000 \\
\hline$T_{0}[$ Rankine $]$ & 900 & 1000 & 900 & 1000 \\
\hline$T_{\infty}[$ Kelvin] & 63.7 & 70.8 & 63.7 & 70.8 \\
\hline$\rho_{\infty}\left[\frac{\mathrm{kg}}{\mathrm{m}^{3}}\right]$ & 0.1951 & 0.1756 & 0.5575 & 0.5017
\end{tabular}

\subsubsection{HIFiRE-1}

In order to determine a surface mesh resolution that produces grid-converged $C_{L}$ and $C_{D}$ values for the HIFiRE-1 payload, four surface meshes were generated using Pointwise GridGen [19] and are shown in Figure 3.1 . 


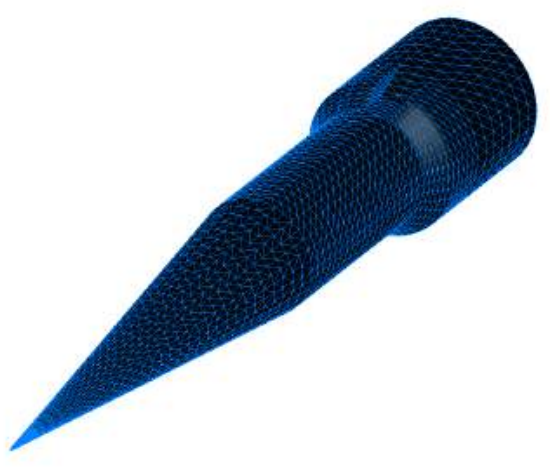

$$
N_{\text {cells }}=7,184
$$

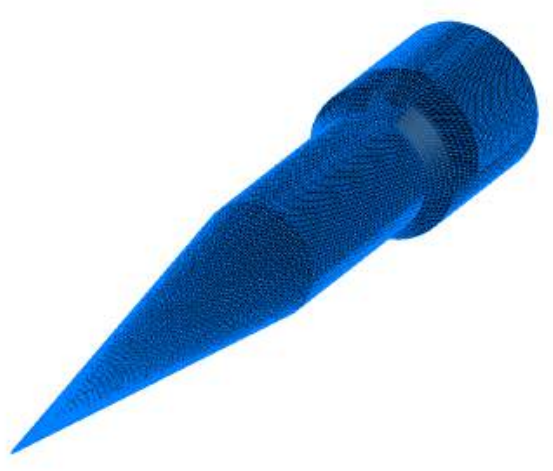

$N_{\text {cells }}=48,224$

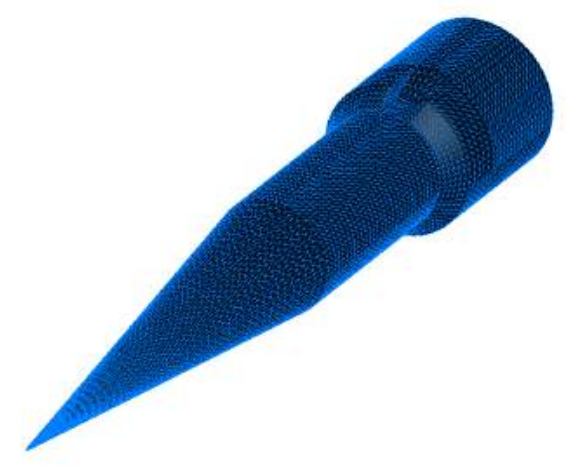

$$
N_{\text {cells }}=24,064
$$

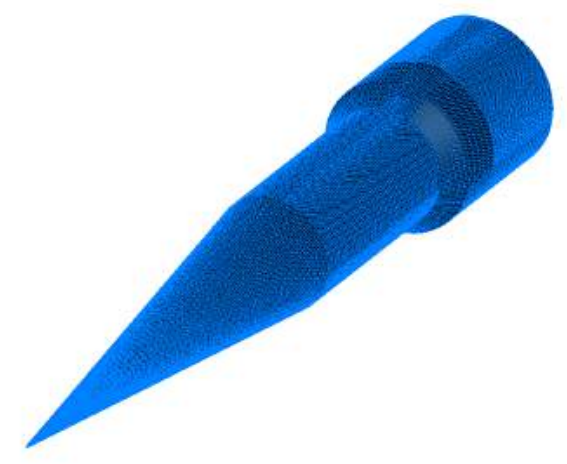

$$
N_{\text {cells }}=80,384
$$

Figure 3.1: CBAero surface meshes for HIFiRE-1 geometry.

Convergence of lift and drag coefficients becomes apparent in Figure 3.2 at resolutions near the 24, 064 cell mesh that was studied. However, a mesh of 80,384 cells, the highest mesh resolution for the HIFiRE-1 CBAero grid convergence study, was used for further analysis at little extra computational cost. 

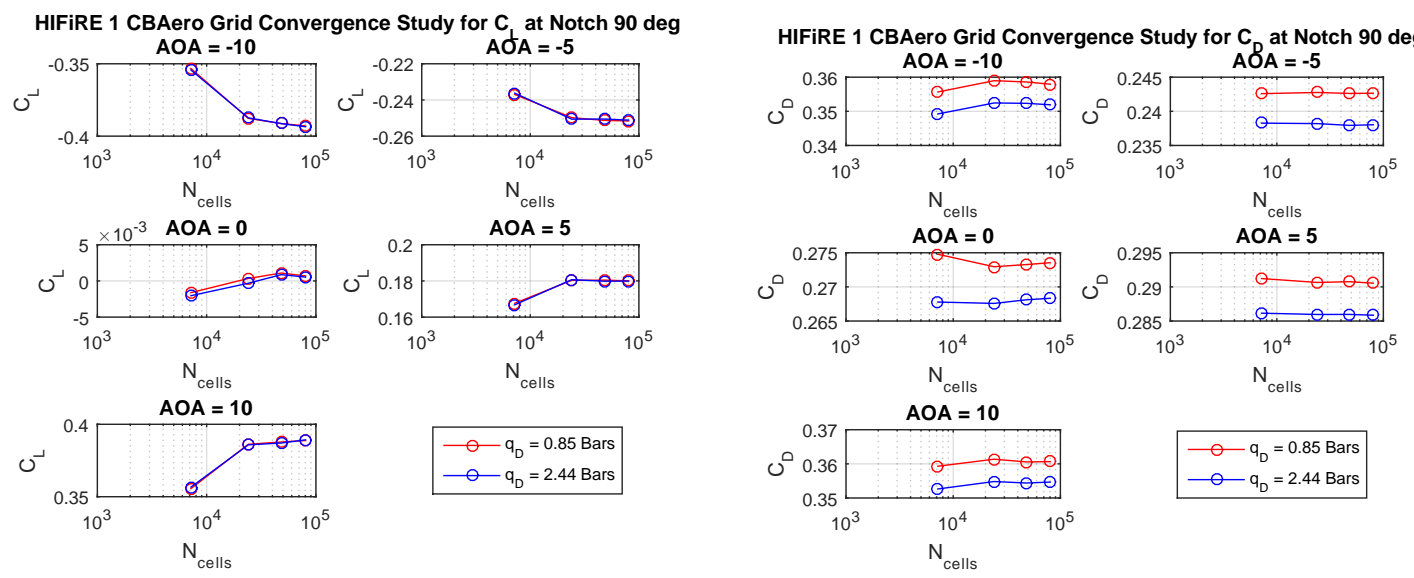

$C_{L}$

$$
C_{D}
$$

Figure 3.2: CBAero Grid Convergence Study for HIFiRE-1 geometry with Notch at 90 deg for various surface meshes

Qualitative viscous approximation results were obtained from CBAero for the HIFiRE-1 model. These results are shown in Figure 3.3 in the form of heating rate distributions on the body at 0 and 10 degrees angle-of-attack for Cases 1 and 4 of Table 3.1 . Contours are reported in $\frac{W}{\mathrm{~cm}^{2}}$. Note that there is higher heating in stagnation regions such as the nose tip and the ramp aft of the constantarea section of the payload. Also, Case 4 shows higher heating than Case 1, which is an expected result due to the higher temperature and pressure conditions of Case 4. 

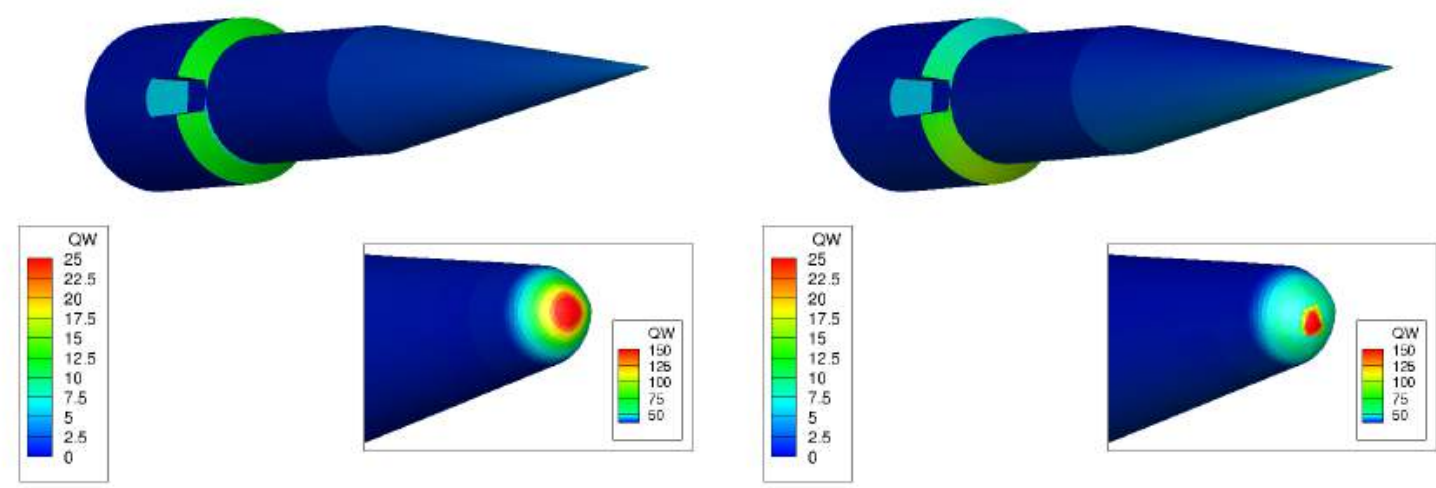

Case $1, \alpha=0$ degrees

Case $1, \alpha=10$ degrees
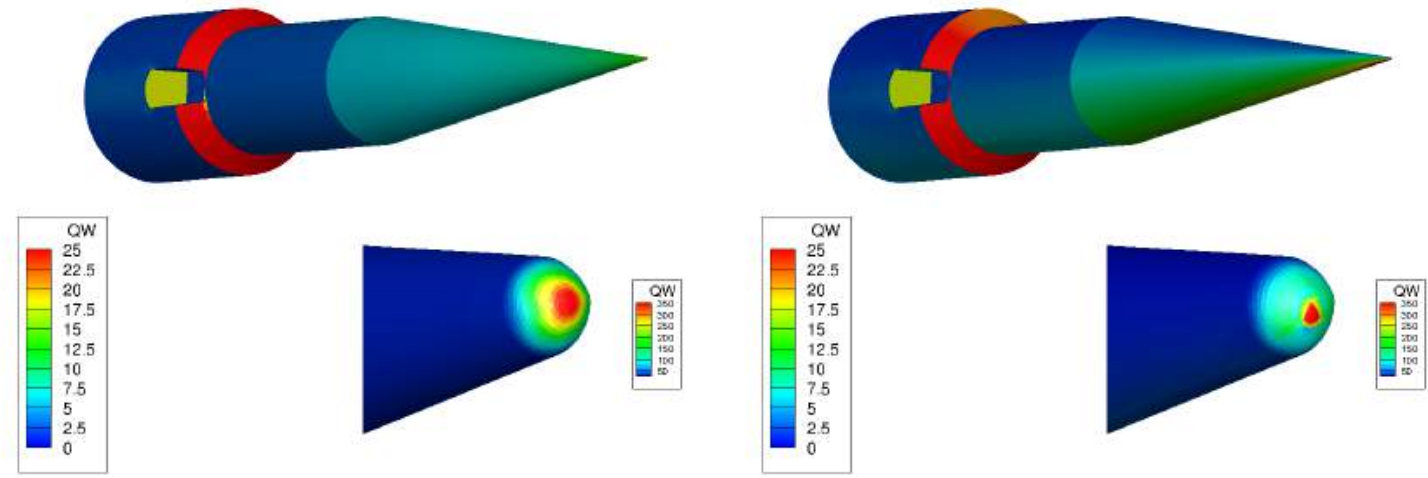

Case $4, \alpha=0$ degrees

Case $4, \alpha=10$ degrees

Figure 3.3: Surface heating rates from CBAero for Notch at $90 \mathrm{deg}$ [6]

\subsubsection{RFSG}

Grid convergence studies were performed with four different surface meshes for the RFSG geometry shown in Figure 3.4. The various surface meshes were once again constructed using Pointwise GridGen [19]. The number of points on the corresponding connectors were changed to maintain consistent, overall refinement to create a true "family" of meshes for the RFSG geometry. 

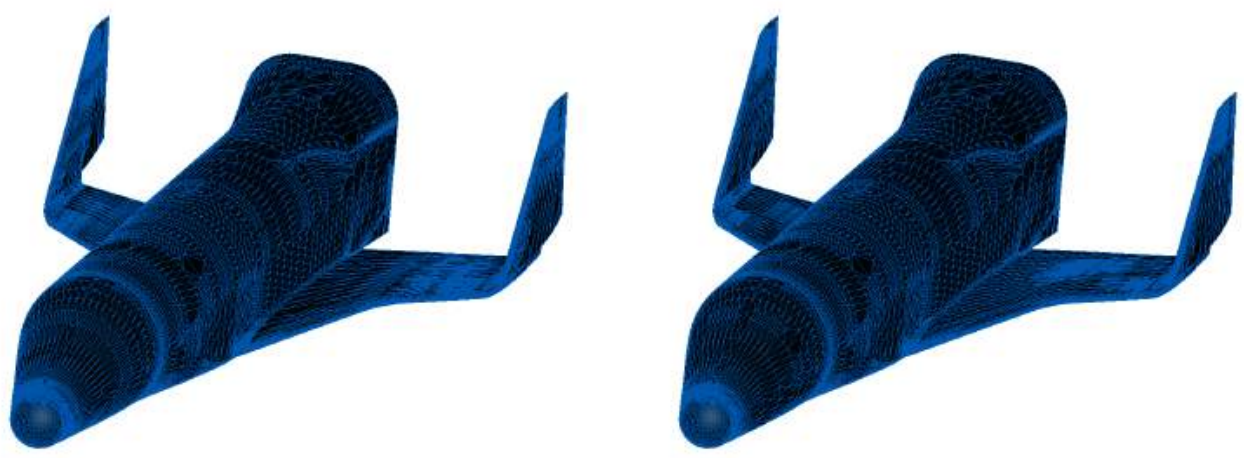

$N_{\text {cells }}=36,934$

$$
N_{\text {cells }}=43,390
$$
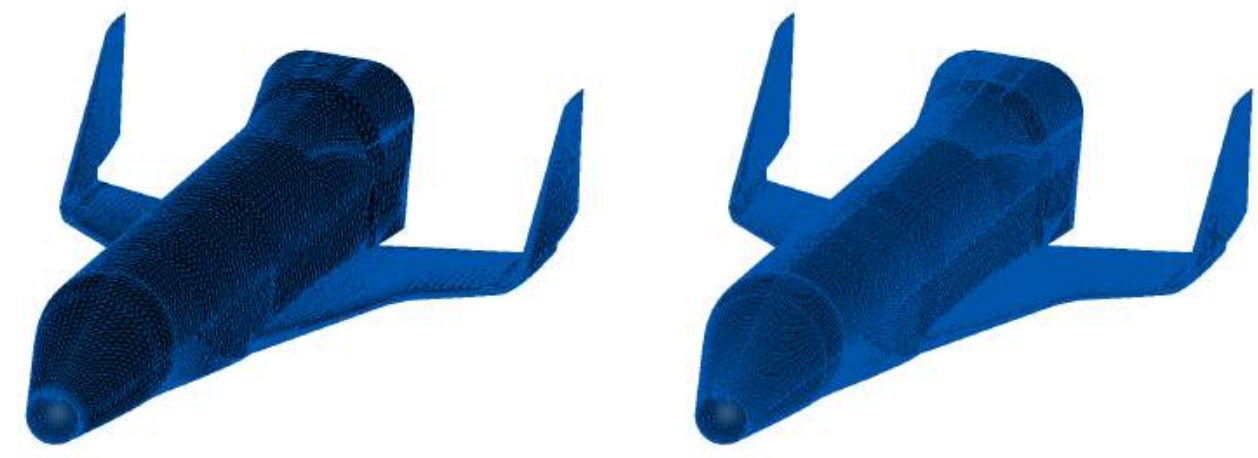

$$
N_{\text {cells }}=78,814
$$

$$
N_{\text {cells }}=189,044
$$

Figure 3.4: CBAero surface meshes for the RFSG geometry [6]

The grid convergence study for the RFSG geometry using CBAero is shown in Figure 3.5, where both $C_{L}$ and $C_{D}$ are shown to converge with increasing number of surface cells. 

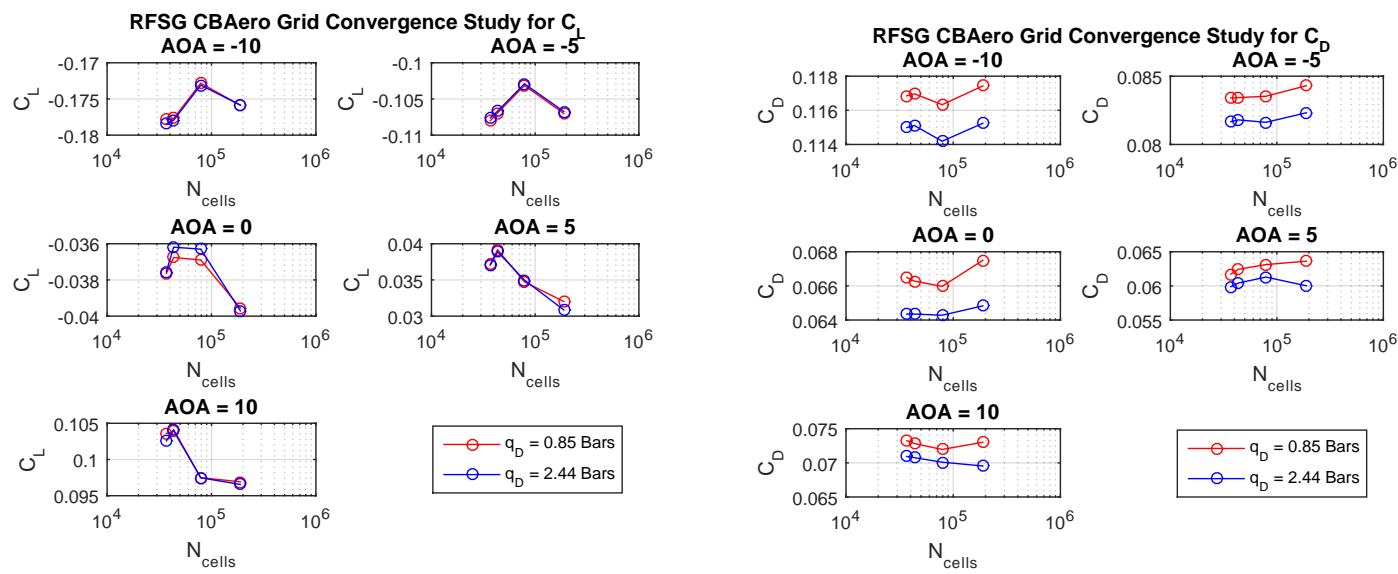

$C_{L}$

$C_{D}$

Figure 3.5: CBAero Grid Convergence Study for RFSG geometry for various surface meshes

Using the most refined mesh having 189, 044 cells, the inviscid $C_{p}$ distribution for the RFSG is shown in Figure 3.6 for Case 1 of Table 3.1, at 0 and -10 degrees AOA. As expected the pressure coefficient increases around the upper nose region where the angle of impact of the flow increases as expected with the Modified Newtonian approximation.

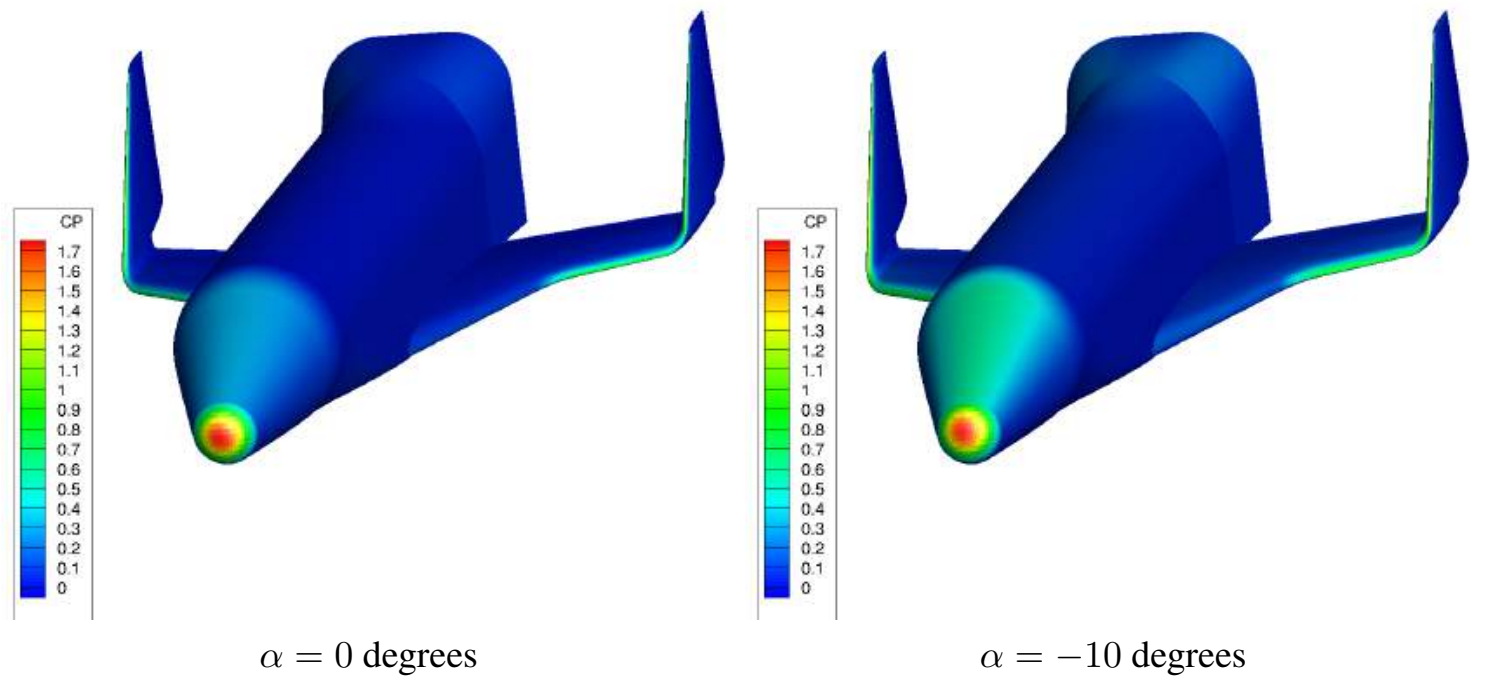

Figure 3.6: $C_{p}$ distribution from CBAero for RFSG [6] 
The results for the viscous approximations using CBAero on the RFSG model are shown in Figure 3.7 in the form of heating rate distributions on the body at 0 and 10 degrees angle-of-attack for Cases 1 and 4 of Table 3.1 Contours are reported in $\frac{W}{\mathrm{~cm}^{2}}$. A heightened heating rate was seen in the nose region and winglets, which is to be expected as these are stagnation regions on this model. Also, Case 4 shows higher heating than Case 1, which is again an expected result due to the higher temperature and pressure condition of Case 4.

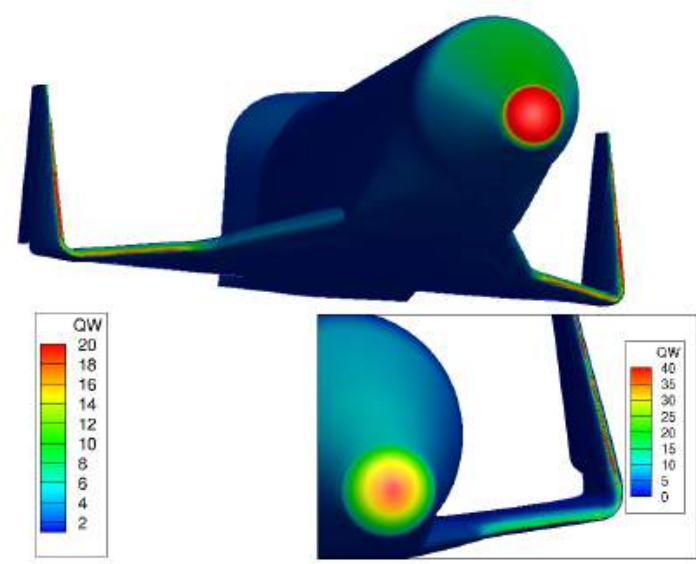

Case $1, \alpha=0$ degrees

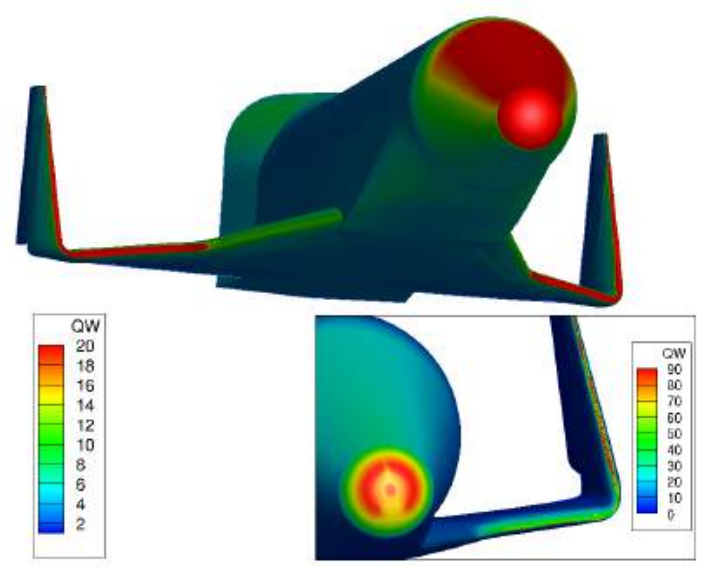

Case $4, \alpha=0$ degrees

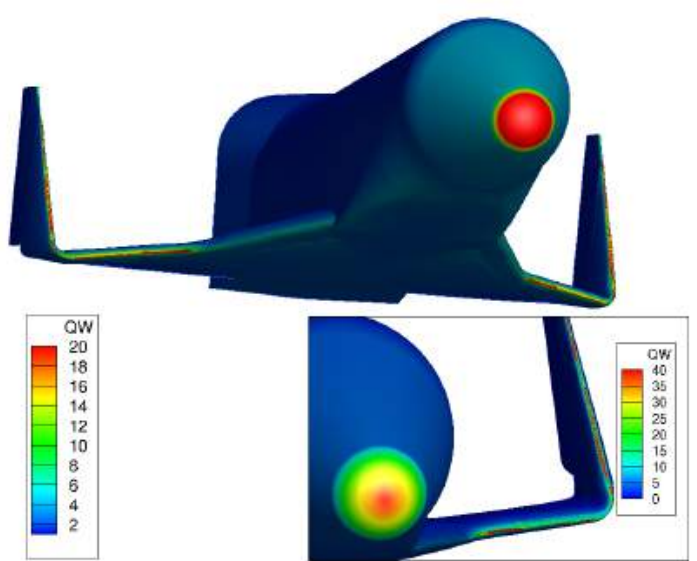

Case $1, \alpha=10$ degrees

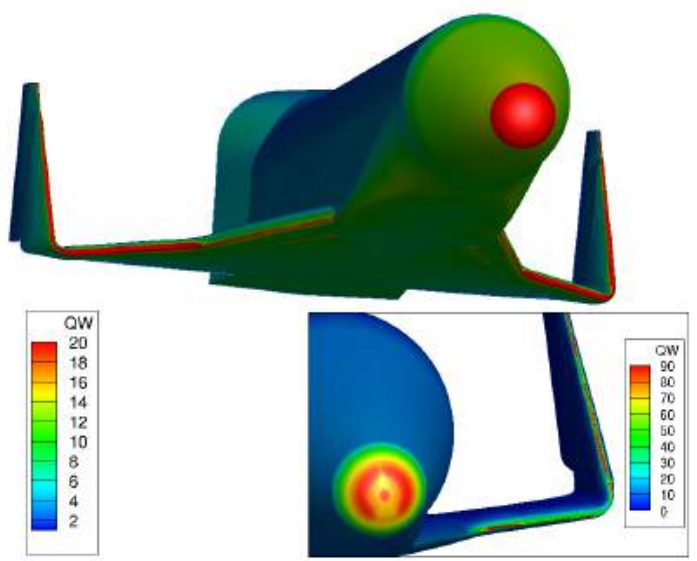

Case $4, \alpha=10$ degrees

Figure 3.7: Surface heating rates from CBAero for RFSG [6] 


\subsubsection{GHV}

A total of five different surface meshes for the GHV geometry were used for a CBAero grid convergence study. These GHV grid models can be seen in Figure 3.8, which were created using Numeca Hexpress Hybrid [20]. Due to the assumption of panel analysis that no cell interacts with one another, a geometry having an inner mold line (IML), such as a pipe, tube, inlet, or engine, causes the panel analysis to output erroneous results for the IML. This is because there is no mechanism, under the former assumption, for the flow to be redirected within an enclosed space, such as reflection or turning of flow within an engine. Therefore the only valid output from CBAero panel analysis for the GHV is from the OML, which includes the fuselage, wings, and tails.

However, the grid convergence study performed for the GHV with CBAero reports $C_{L}$ and $C_{D}$ for all cells of the geometry, including OML and IML. This was done to simplify the analysis and to prevent the complexity of isolating the OML from the IML. It is assumed in this grid convergence study that if $C_{L}$ and $C_{D}$ of the OML and IML have small changes for increasing surface mesh resolution, then the corresponding surface mesh is of high enough resolution. A later comparison of $C_{p}$ at a cross-section of the wing between CBAero and Cart3D (which accounts for flow interaction) shows good agreement between the $C_{p}$ distributions of the two codes, giving confidence in this grid convergence study. 

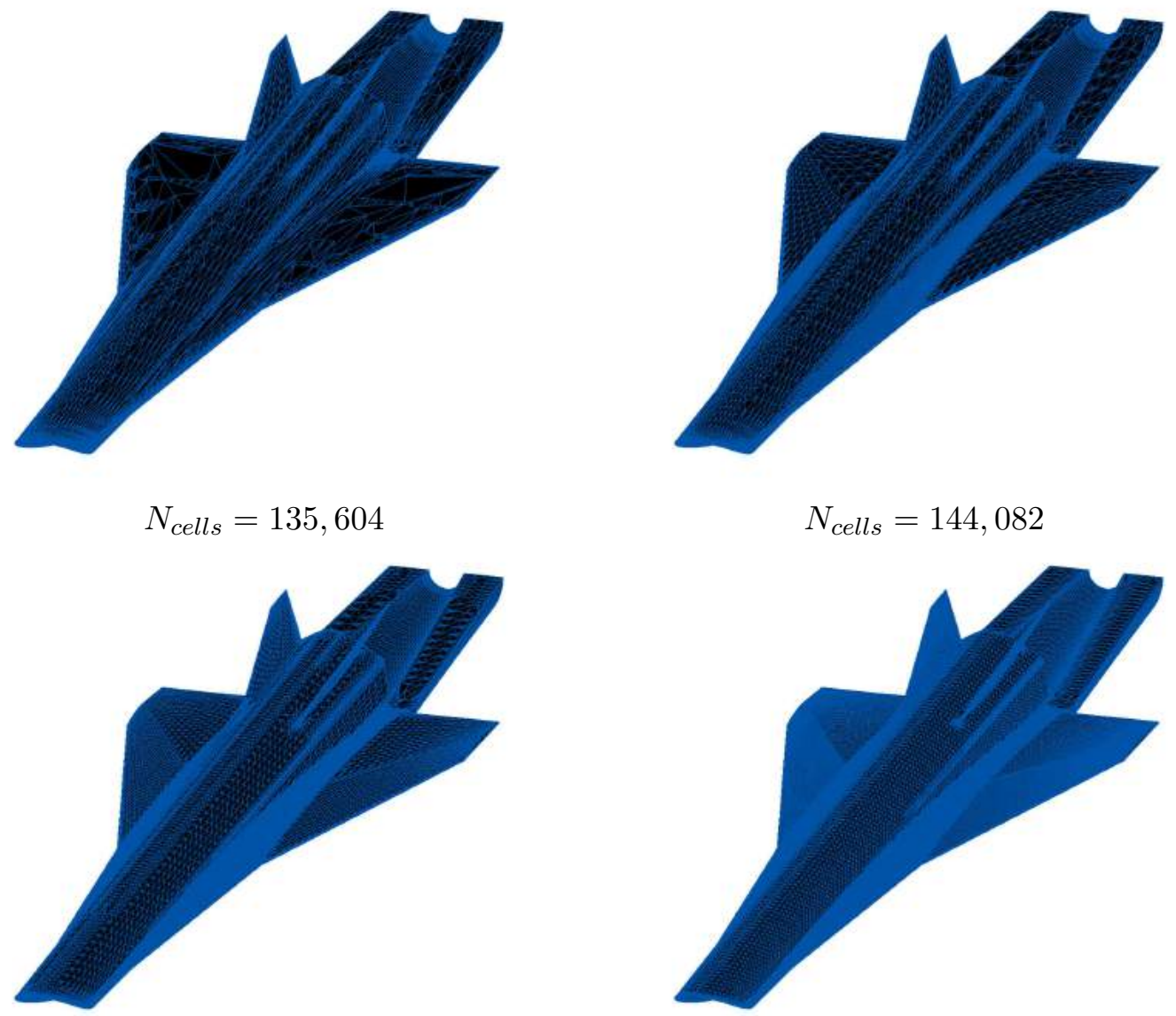

$N_{\text {cells }}=174,018$

$N_{\text {cells }}=221,454$

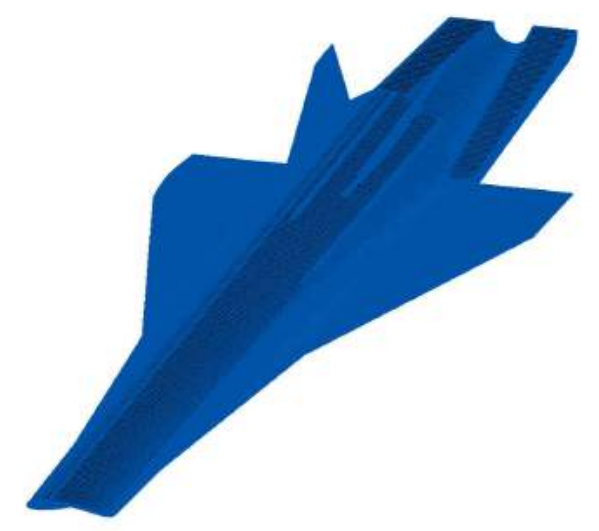

$$
N_{\text {cells }}=478,394
$$

Figure 3.8: CBAero surface meshes for the GHV geometry [6] 
The results of the CBAero grid convergence study for the GHV are shown in Figure 3.9. From the grid convergence study, a surface mesh of 221,454 cells was determined to produce gridconverged values of $C_{L}$ and $C_{D}$ for the GHV.
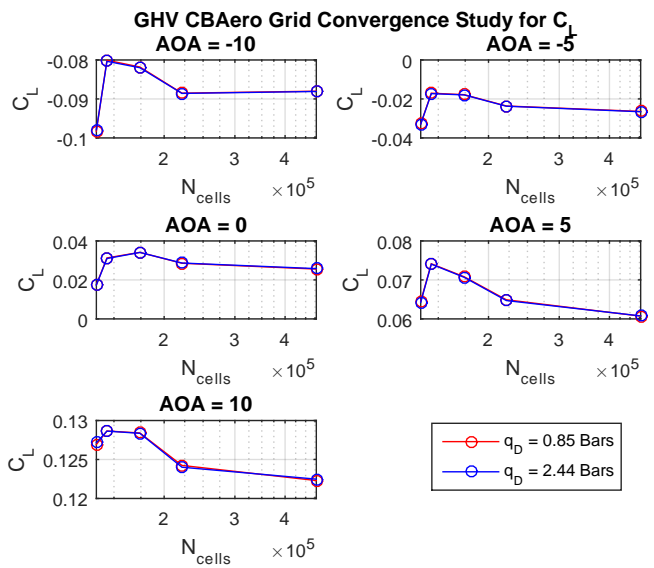

$C_{L}$
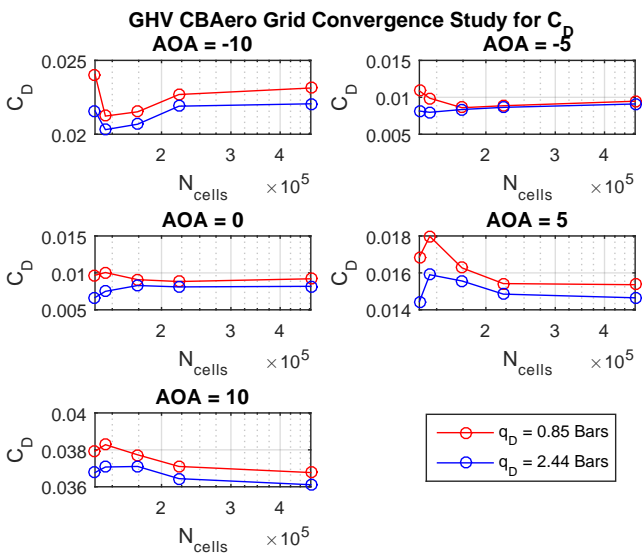

$-\mathrm{q}_{\mathrm{D}}=0.85$ Bars $\longrightarrow q_{D}=2.44$ Bars

$C_{D}$

Figure 3.9: CBAero Grid Convergence Study for GHV geometry for various surface meshes

This surface mesh was used to run CBAero to produce inviscid results (again reporting OML and IML to reduce analysis complexity), which are displayed in the form of $C_{p}$ contours in Figure 3.10 again for Case 1 of Table 3.1, at -10 and 0 degrees $A O A$. 


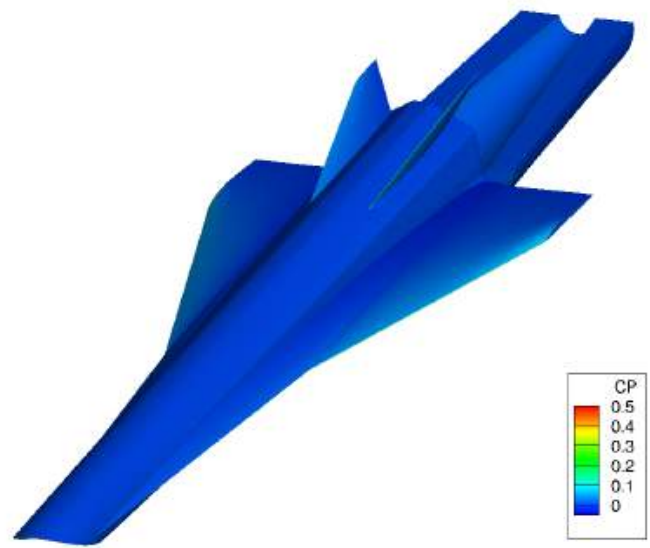

$\alpha=0$ degrees

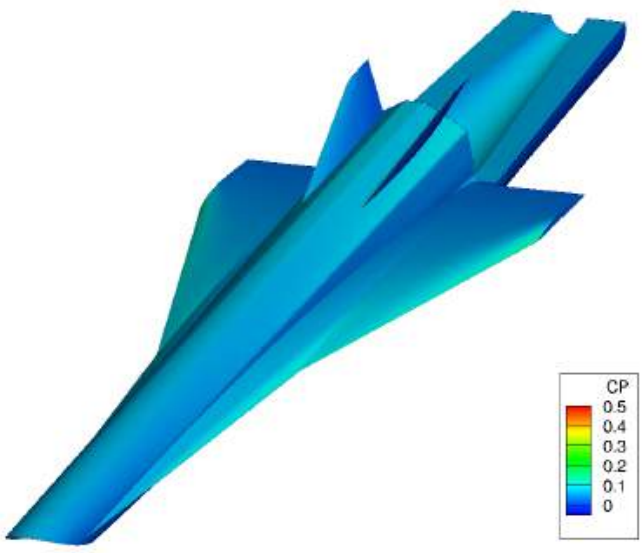

$\alpha=-10$ degrees

Figure 3.10: $C_{p}$ distribution from CBAero for GHV [6]

\subsubsection{Limitations in CBAero Analysis}

Some difficulties arose in the analysis of the HIFiRE-1, RFSG and GHV geometries when using CBAero. Firstly, providing a surface mesh that was not closed, or water-tight, caused issues with the viscous solver of CBAero. If the mesh retained open seams or holes, the solver could not easily integrate over the body to produce friction and heating approximations. Secondly, due to the assumption of a panel analysis discussed in Section 3.1.3. CBAero is rendered invalid as an analysis tool for a full configuration that includes an engine, such as the GHV. If only the OML is considered, the panel analysis is valid. However, a mesh that excludes its IML is then no longer water-tight. Thus, no viscous results are shown for the GHV. Finally, initial results of wall temperature distributions for the HIFiRE-1 and RFSG geometries showed signs of either over-predicting temperature by a large margin or indicated asymmetry in the distributions even when no side-slip was involved. Causes for the over-prediction and asymmetries in the temperature distributions were 
not determined during the period of performance of this effort, and therefore temperature results are excluded from this work.

\subsection{CART3D Euler Analysis}

Medium-fidelity computational flow simulations were performed using Cart3D, a software suite employing automated and error-adapted [16, 17] Cartesian volume mesh generation to solve the Euler flow equations. While monitoring a user-specified functional, such as lift, drag, or pitching moment, Cart3D uses "adjoint-weighted" error estimation [21, 16] to cluster nodes in regions of the flow-field where error in the flow solution degrades the accuracy of the chosen functional. In this effort, the value of pitching moment coefficient, $C_{M}$, was monitored to drive adjoint-weighted mesh refinement for the off-body flow-field for the HIFiRE-1 payload. A combined functional $0.6 C_{D}+0.4 C_{L}$ was used to drive the adaptations for the RFSG and GHV geometries. Using the mesh adaptation capability of Cart3D, grid convergence studies were performed for the HIFiRE-1, RFSG and GHV models which are shown next in their respective Subsections.

Since CART3D outputs the inviscid flow velocities $u, v$ and $w$ the following conversion must be made to have the results in terms of free stream Mach number, shown in Equation 3.2 .

$$
M=\sqrt{\frac{u^{2}+v^{2}+w^{2}}{\frac{\gamma p}{\rho}}}
$$

In order to obtain the coefficient of pressure from the computed inviscid pressure, $p$ and known free stream pressure, $p_{\infty}$, the following relationship is used:

$$
C_{p}=\frac{p-p_{\infty}}{q_{\infty}}=\frac{\frac{p}{p_{\infty}}-1}{\frac{q_{\infty}}{p_{\infty}}}=\frac{\frac{p}{p_{\infty}}-1}{\frac{1}{2} \gamma M^{2}}
$$




\subsubsection{HIFiRE-1}

A grid convergence study was conducted with three initial volume grids staged through seven different adaptation levels. One of the surface meshes used for building the volume grids is shown in Figure 3.11. The volume grids with higher resolution near the nose is shown in Figures 3.12 and 3.13 Note that an added benefit to this meshing approach is the high resolution of the shock structure surrounding the body. A downside, however, is that the cell count increases rapidly with each adaptation, since the starting mesh tends to be much larger. Integrated quantities of $C_{L}, C_{D}$, and $C_{M}$ from the study are shown in Figures 3.143 .16 . The residuals were only reduced to approximately two orders of magnitude in this study. Time for this effort ran out before a root cause for the low residual reduction could be determined. Even though the residuals are higher than preferred in CFD studies (desiring reductions to at least four orders of magnitude for hypersonic flow and preferably eight orders of magnitude in most cases), the integrated quantities do not appear to suffer from this. In fact, the coefficients monitored reach values that experience negligible changes at eight and nine adaptations for initial volume grids with cell counts greater than roughly 40, 000 Cartesian cells.

Resulting flow features for the first case in Table 4.1 are shown in Section 4.1. 


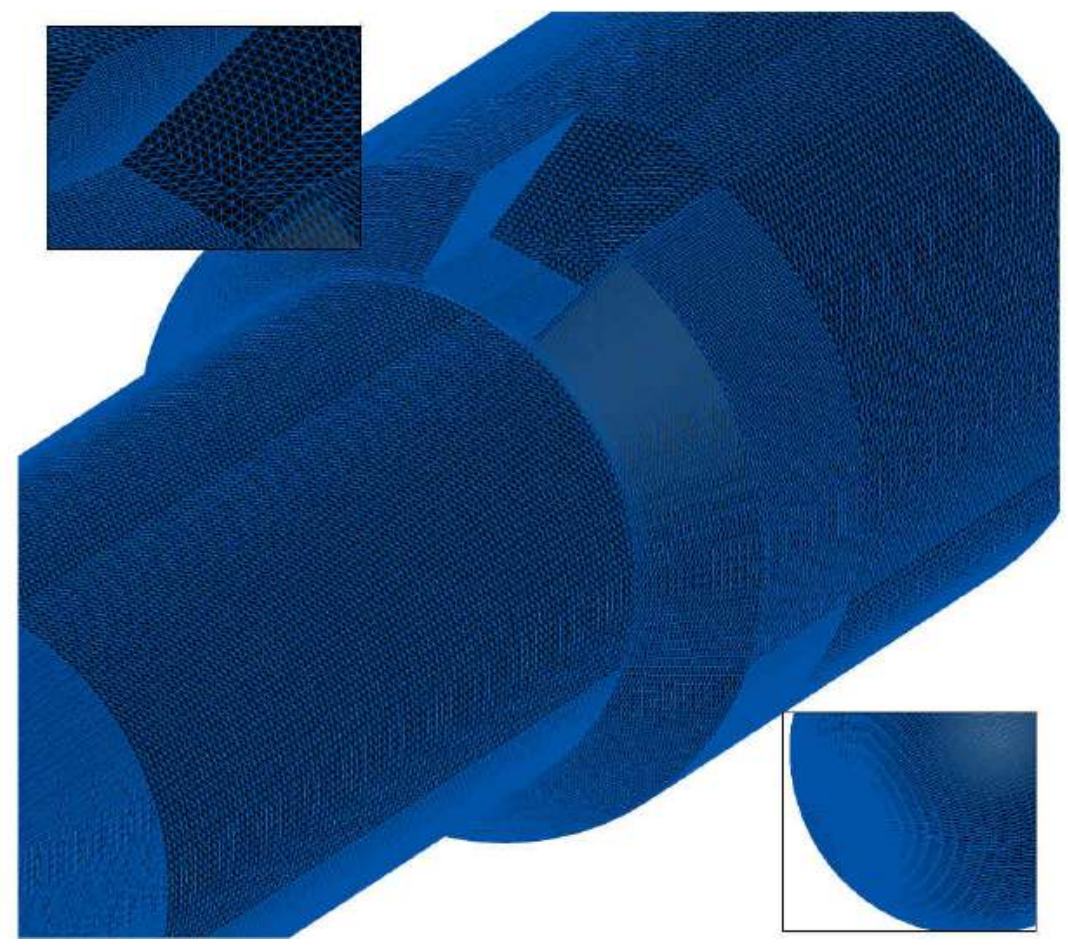

Figure 3.11: HIFiRE-1 Surface Mesh used for CART3D.

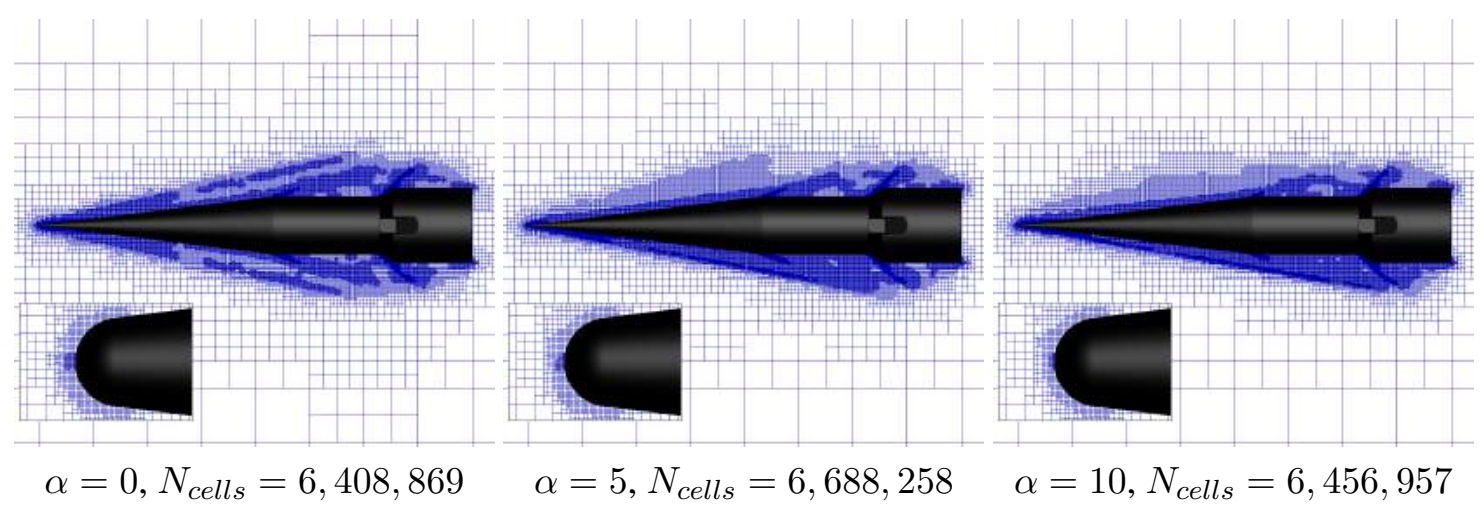

Figure 3.12: CART3D Volume Meshes through $y$-cut plane. 


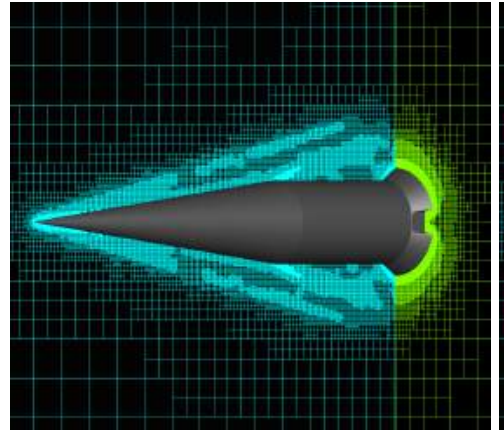

$\alpha=0, N_{\text {cells }}=6,408,869$

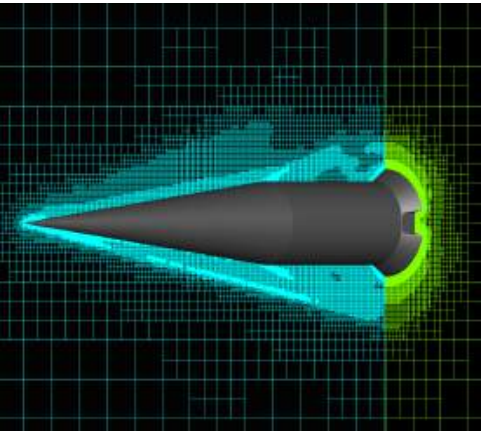

$\alpha=5, N_{\text {cells }}=6,688,258$

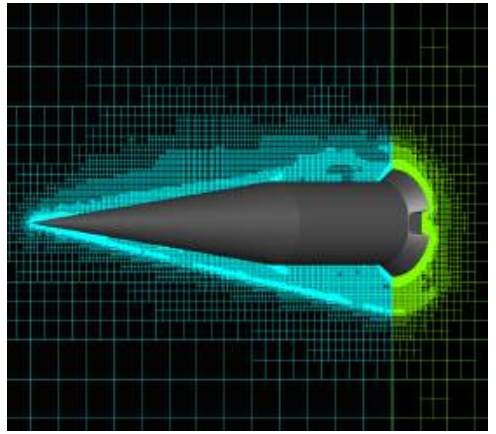

$\alpha=10, N_{\text {cells }}=6,456,957$

Figure 3.13: CART3D Volume Meshes through $x$ and $y$-cut plane. 

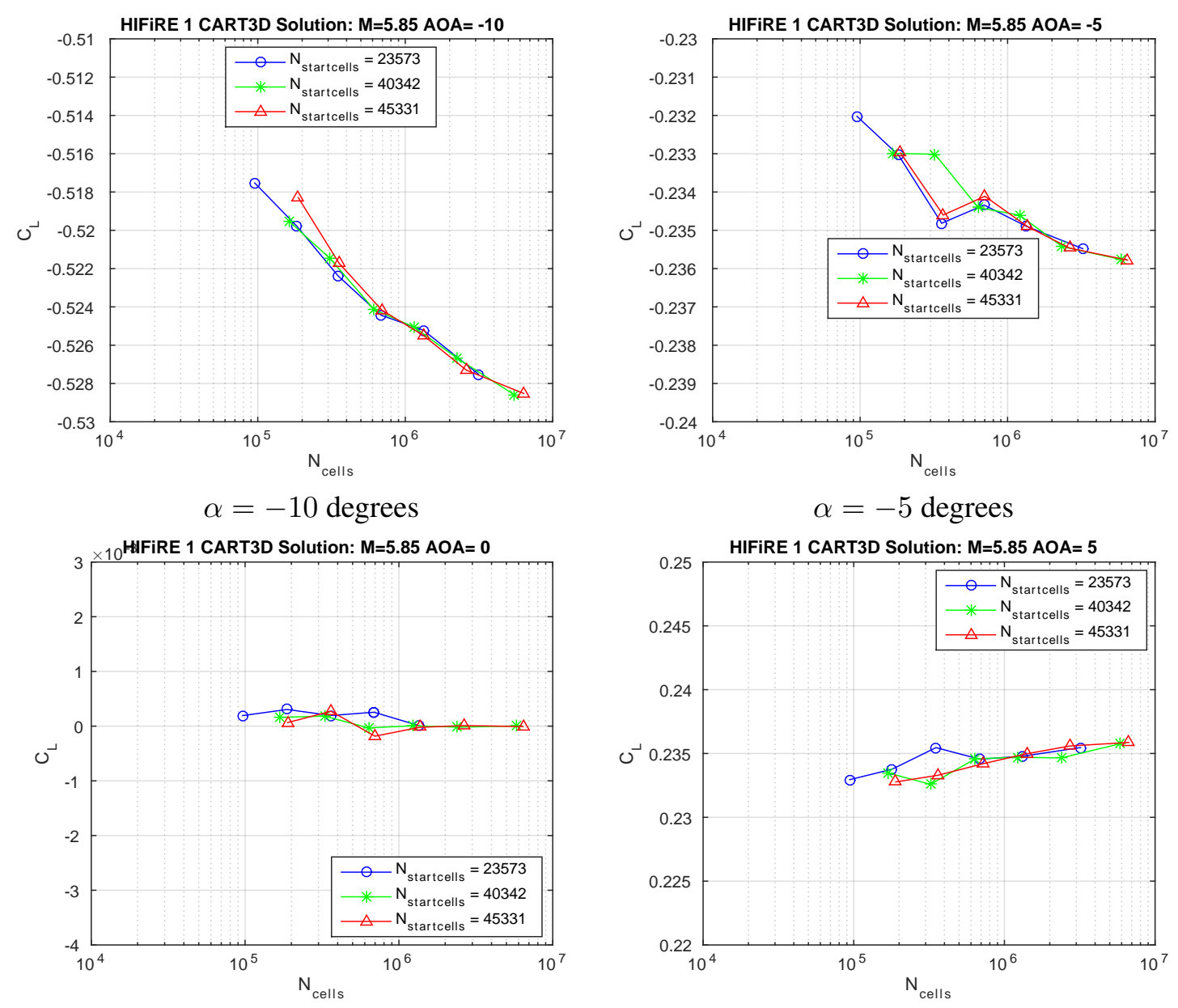

$\alpha=0$ degrees

$\alpha=5$ degrees

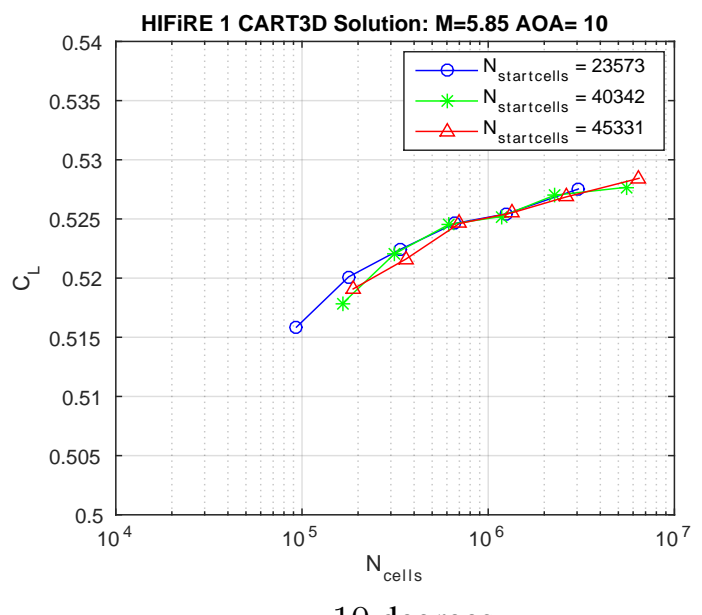

$\alpha=10$ degrees

Figure 3.14: CART3D Grid Convergence Study for HIFiRE 1 geometry for $C_{L}$. 

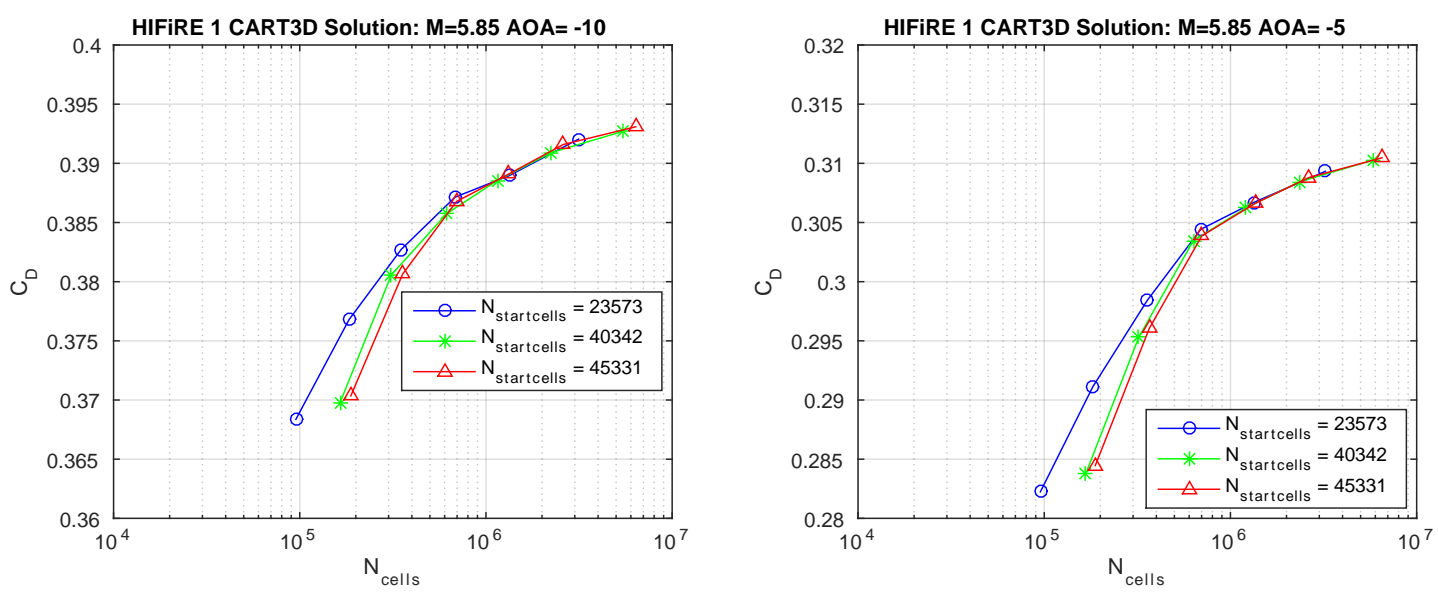

$\alpha=-10$ degrees

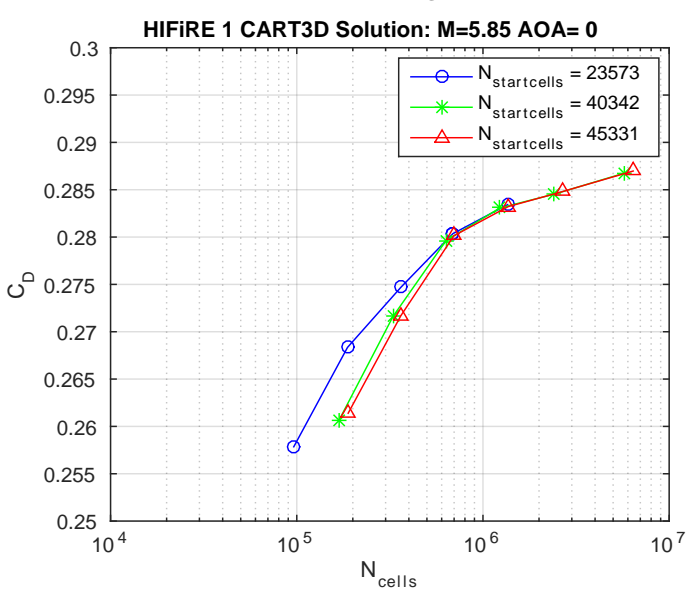

$\alpha=-5$ degrees

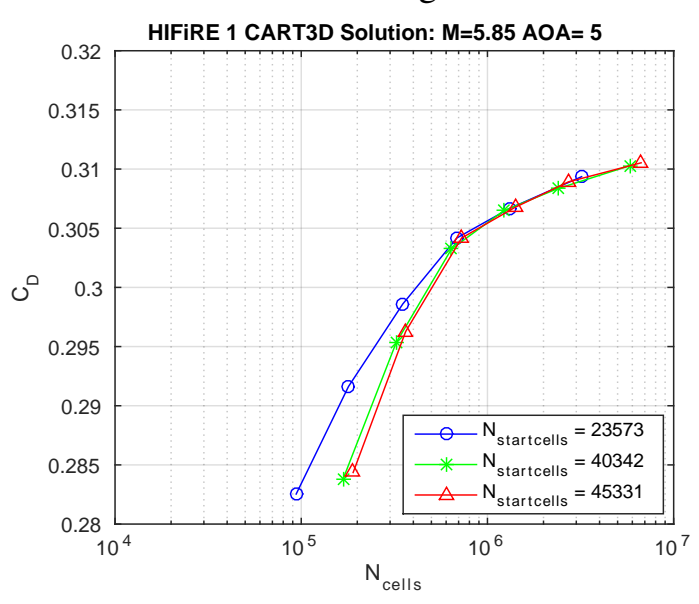

$\alpha=0$ degrees

$\alpha=5$ degrees

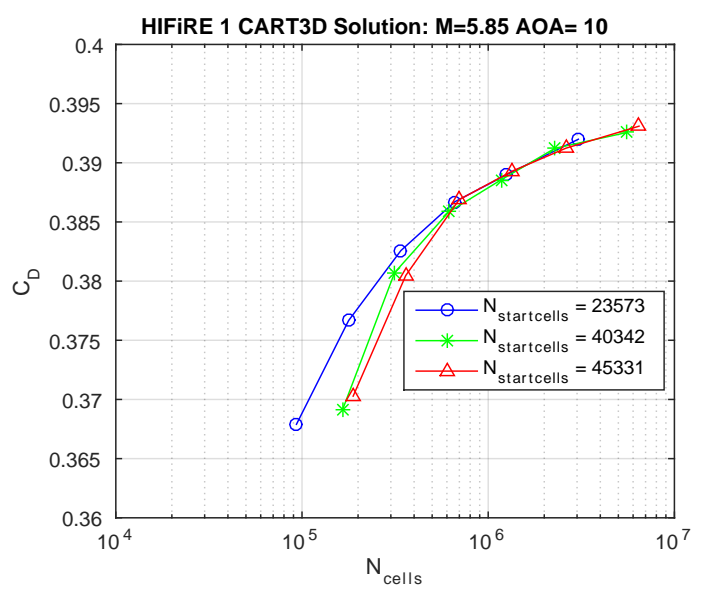

$\alpha=10$ degrees

Figure 3.15: CART3D Grid Convergence Study for HIFiRE 1 geometry for $C_{D}$. 

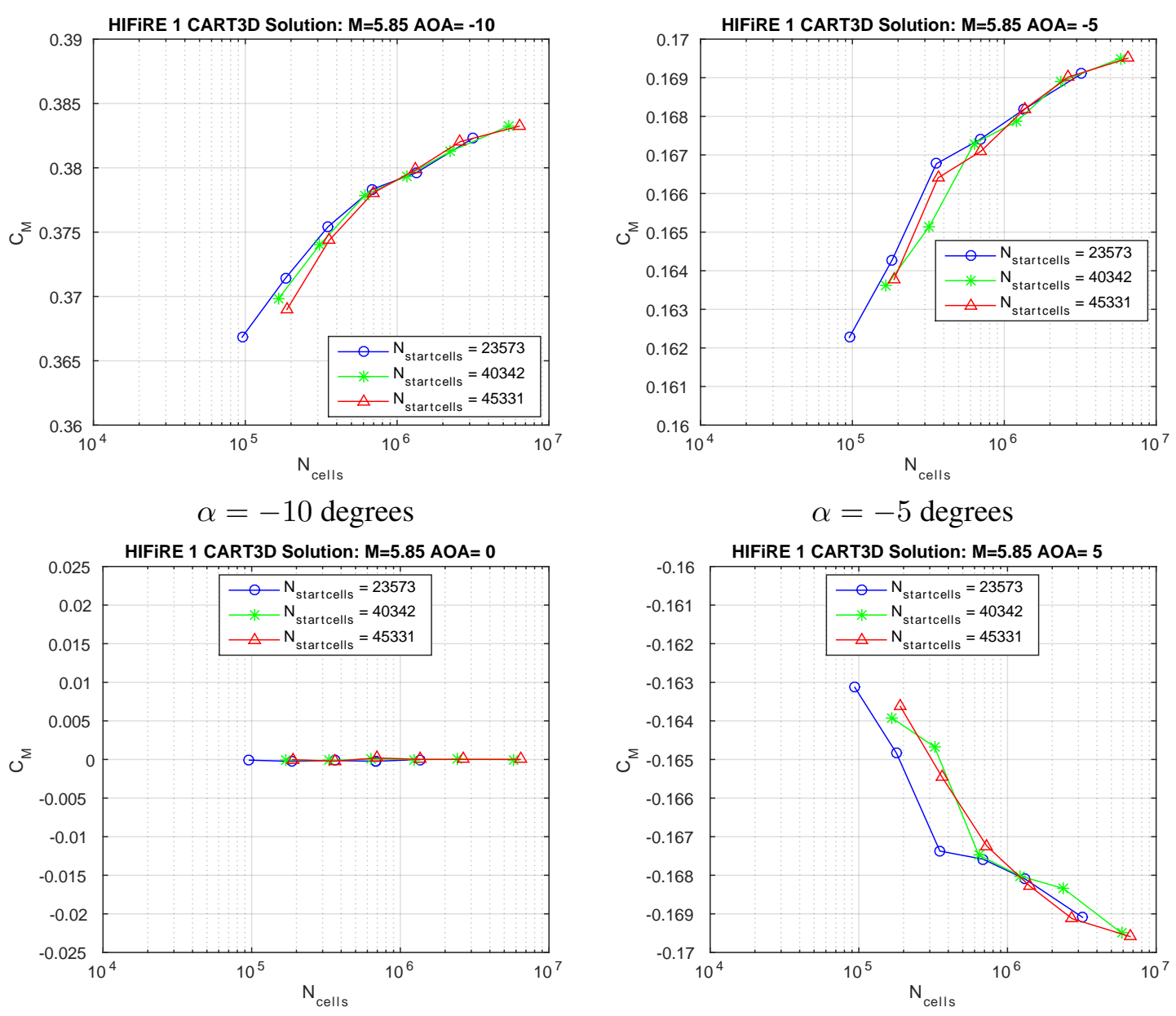

$\alpha=0$ degrees

$\alpha=5$ degrees

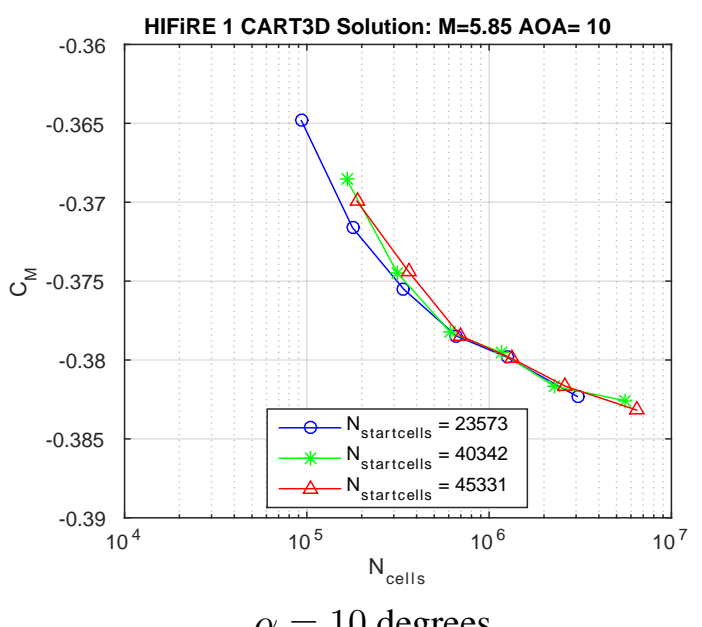

$\alpha=10$ degrees

Figure 3.16: CART3D Grid Convergence Study for HIFiRE 1 geometry for $C_{M}$. 


\subsubsection{RFSG}

To conduct the Cart3D grid convergence study of the RFSG, four initial volume meshes were staged to reach five different levels of mesh adaptation. A surface mesh of 461,492 cells was first created to input into Cart3D's automatic Cartesian mesh generator, cubes. The surface mesh is shown in Figure 3.17. Cubes then takes the surface mesh and creates an initial volume grid, denoted as $N_{\text {startcells }}$, of Cartesian cells such that the surface mesh provides a wall boundary that defines the geometry at hand. Adjoint-adapted solutions were then obtained from Cart3D by staging the initial volume mesh from five to nine adaptations. The volume meshes of three different angles-ofattack after nine adaptations for the RFSG are showcased in Figures 3.18 and 3.19. Note how the adaptation of the mesh is capable of resolving flow features and highly contoured regions, such as the leading bow shock and nose of the vehicle. Solutions shown in this section are shown on the mesh obtained from $N_{\text {startcells }}=274,711$ and 9 adaptation cycles.

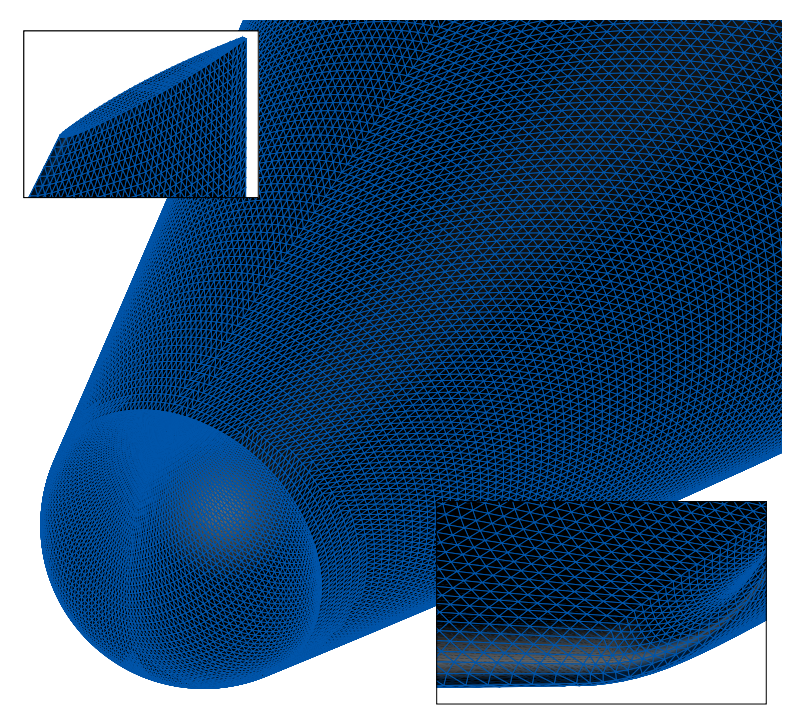

Figure 3.17: RFSG Surface Mesh with $N_{\text {cells }}=461,492$. 


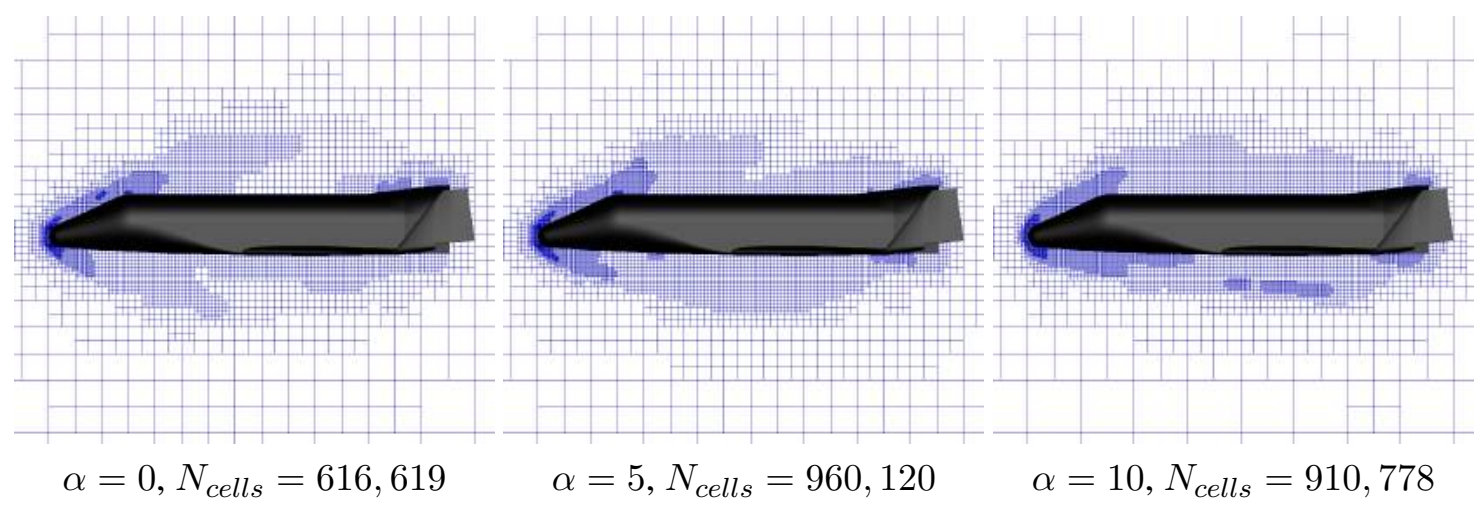

Figure 3.18: RFSG Volume Meshes through $y$ - cut plane.

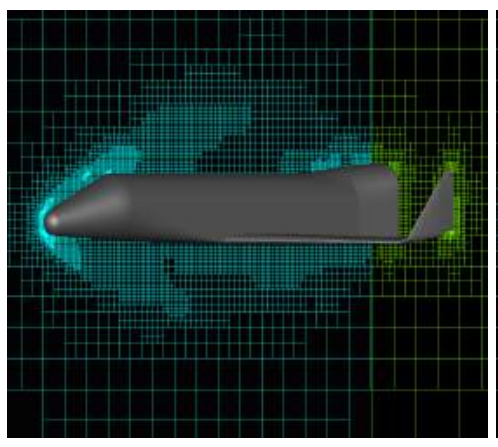

$\alpha=0, N_{\text {cells }}=616,619$

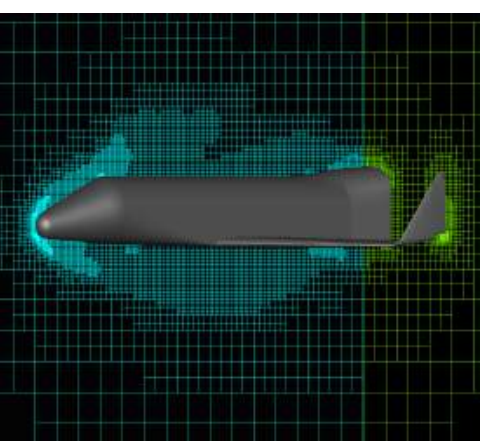

$\alpha=5, N_{\text {cells }}=960,120$

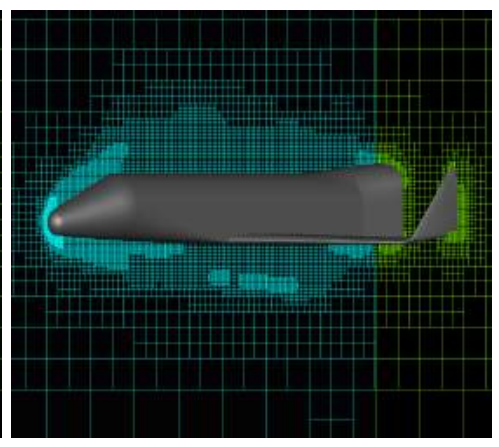

$\alpha=10, N_{\text {cells }}=910,778$

Figure 3.19: RFSG Volume Meshes through $x$ and $y$-cut plane [6]

The convergence histories for the RFSG geometry is shown in Figure 3.20, where by the 9th adaptation the residual is shown to drop by approximately 3-4 orders of magnitude. 


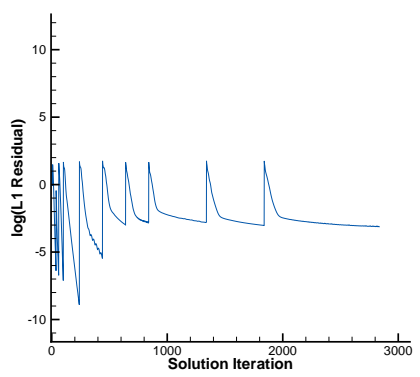

$M=6, \alpha=0$ degrees

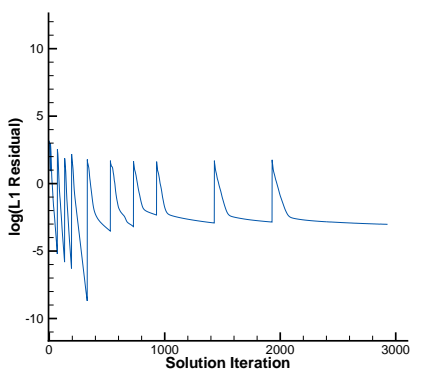

$M=6, \alpha=5$ degrees

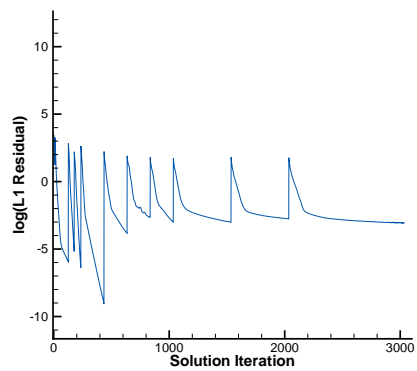

$M=6, \alpha=10$ degrees

Figure 3.20: RFSG convergence histories after 9 adaptations.

Stagnation regions can be readily seen at the nose and wing leading edges in the surface $C_{p}$ contours, Figure 3.21, while strong flow interactions are apparent near the nose in the Mach contour and density fields, see Figures 3.22 and 3.23 , respectively.

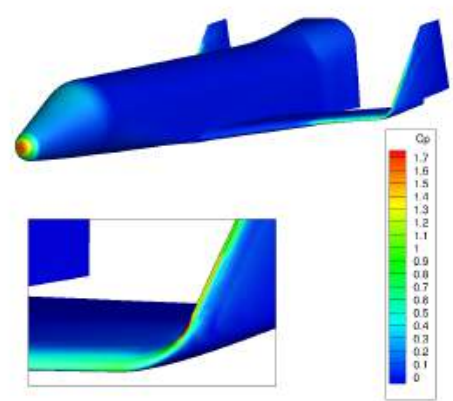

$M=6, \alpha=0$ degrees

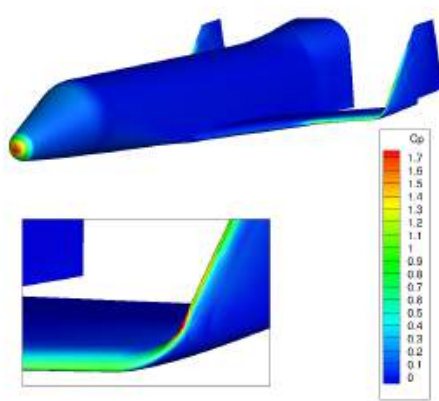

$M=6, \alpha=5$ degrees

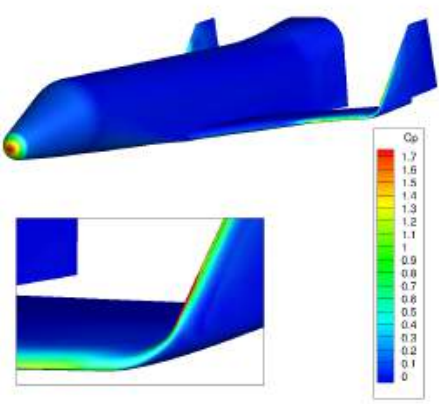

$M=6, \alpha=10$ degrees

Figure 3.21: RFSG $C_{p}$ contours. 


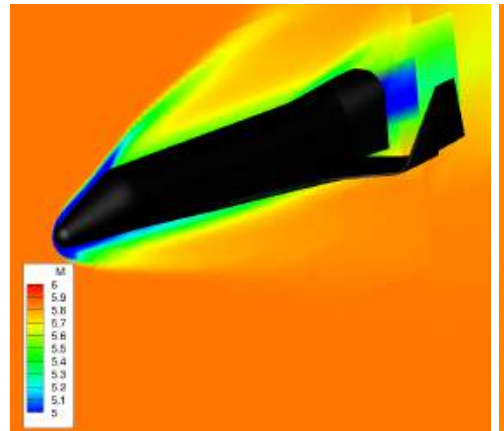

$M=6, \alpha=0$ degrees

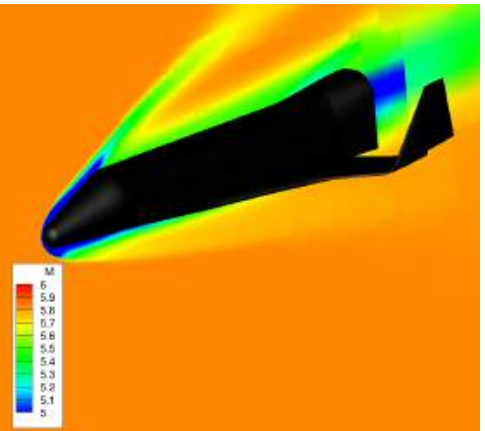

$M=6, \alpha=5$ degrees

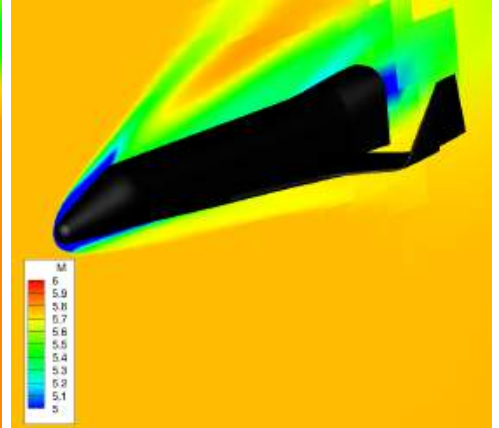

$M=6, \alpha=10$ degrees

Figure 3.22: RFSG Mach contours.

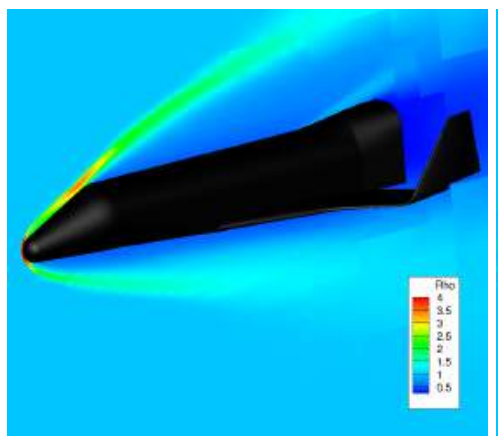

$M=6, \alpha=0$ degrees

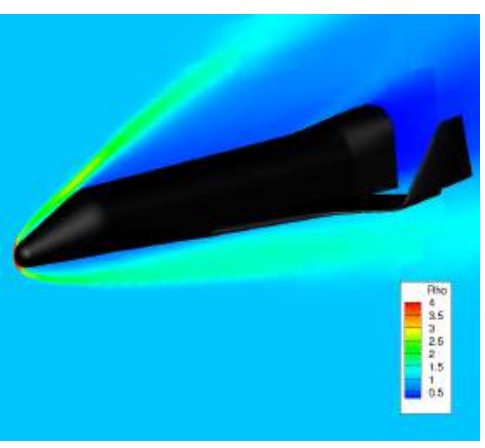

$M=6, \alpha=5$ degrees

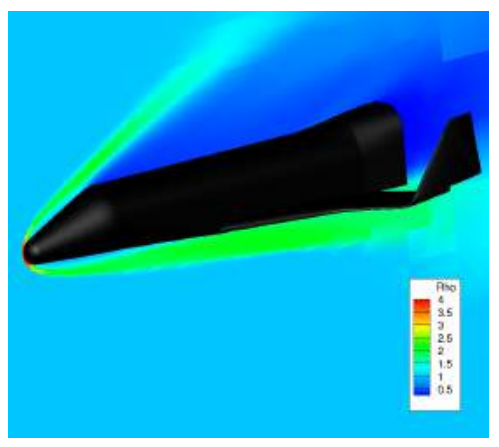

$M=6, \alpha=10$ degrees

Figure 3.23: CART3D $\rho$ contours.

A grid convergence study was completed for the RFSG geometry to ensure a grid converged solution. The convergence of $C_{L}, C_{D}$ and $C_{M}$ are shown in Figures 3.24 3.26. Various initial mesh sizes, shown as $N_{\text {startcells }}$, were evaluated which increased in size with each adaptation cycle. Grid convergence is reached near about one million cells, corresponding to roughly seven to nine adaptations for starting meshes greater than $N_{\text {startcells }}=5,500$ cells. 

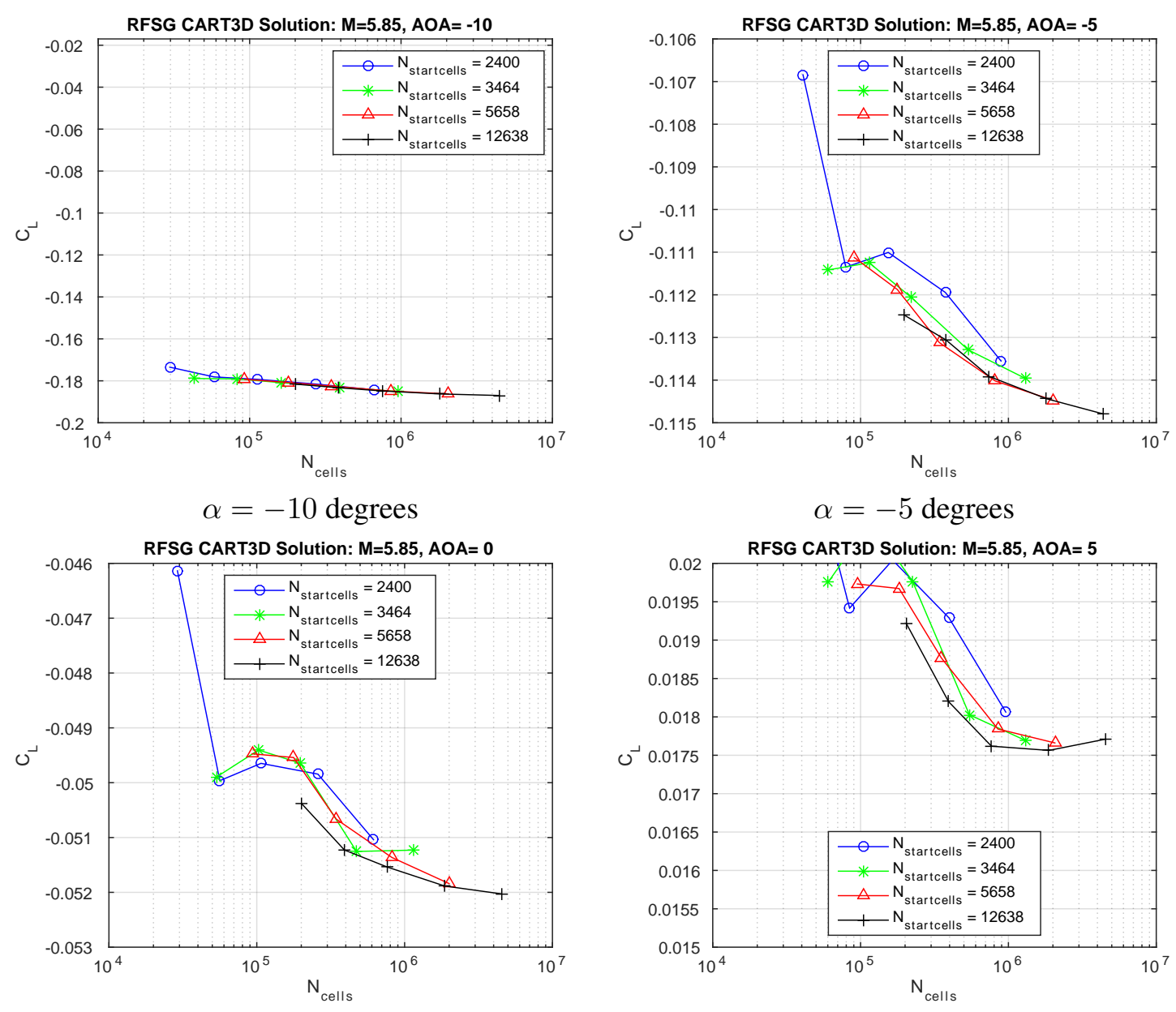

$\alpha=0$ degrees

$\alpha=5$ degrees

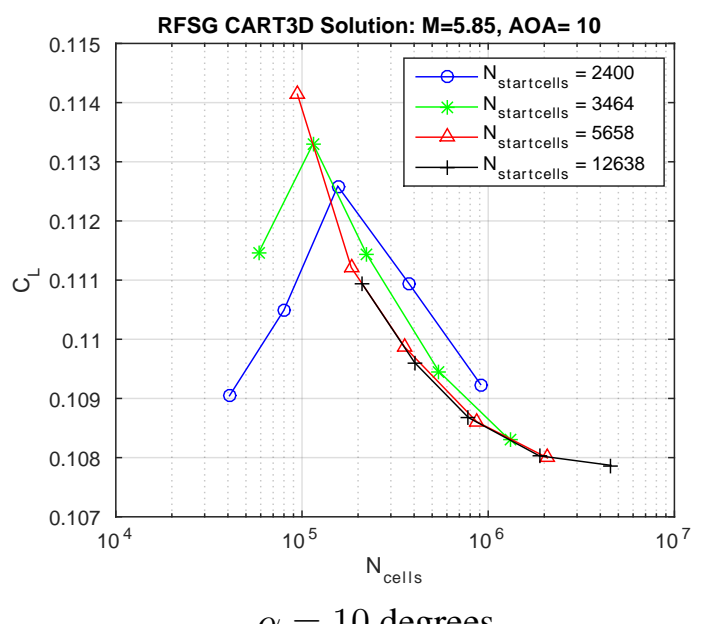

$\alpha=10$ degrees

Figure 3.24: CART3D Grid Convergence Study for RFSG geometry for $C_{L}$. 

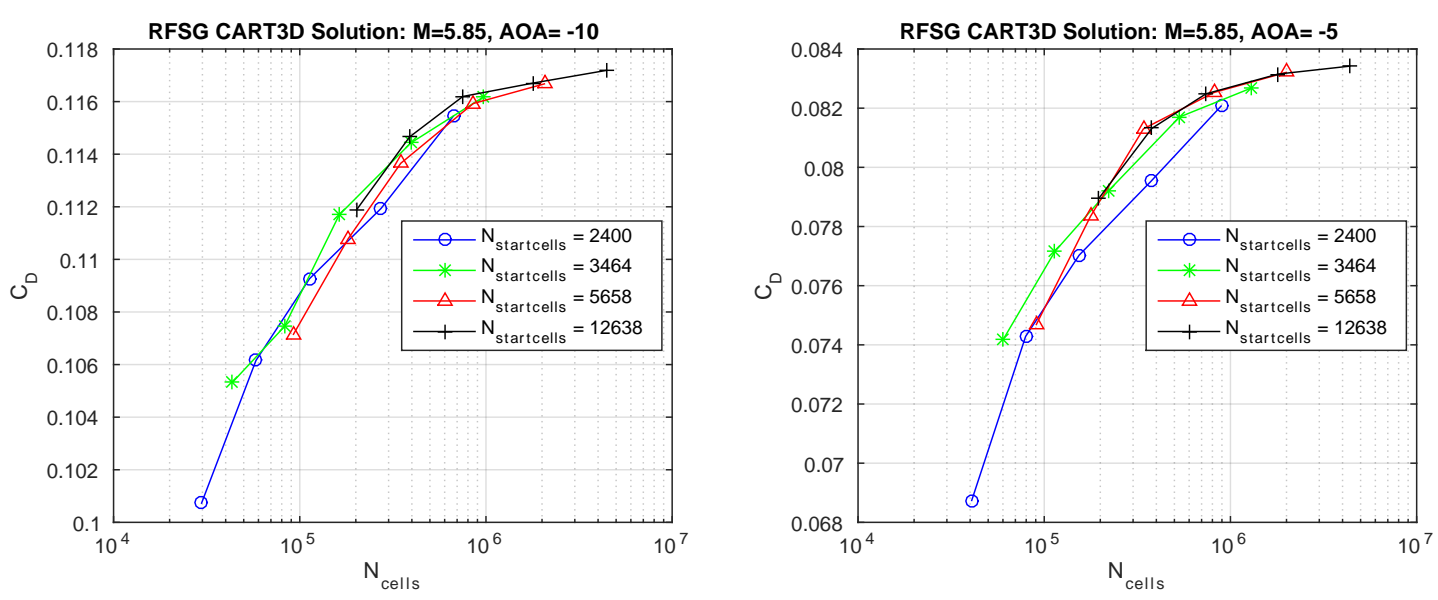

$\alpha=-10$ degrees
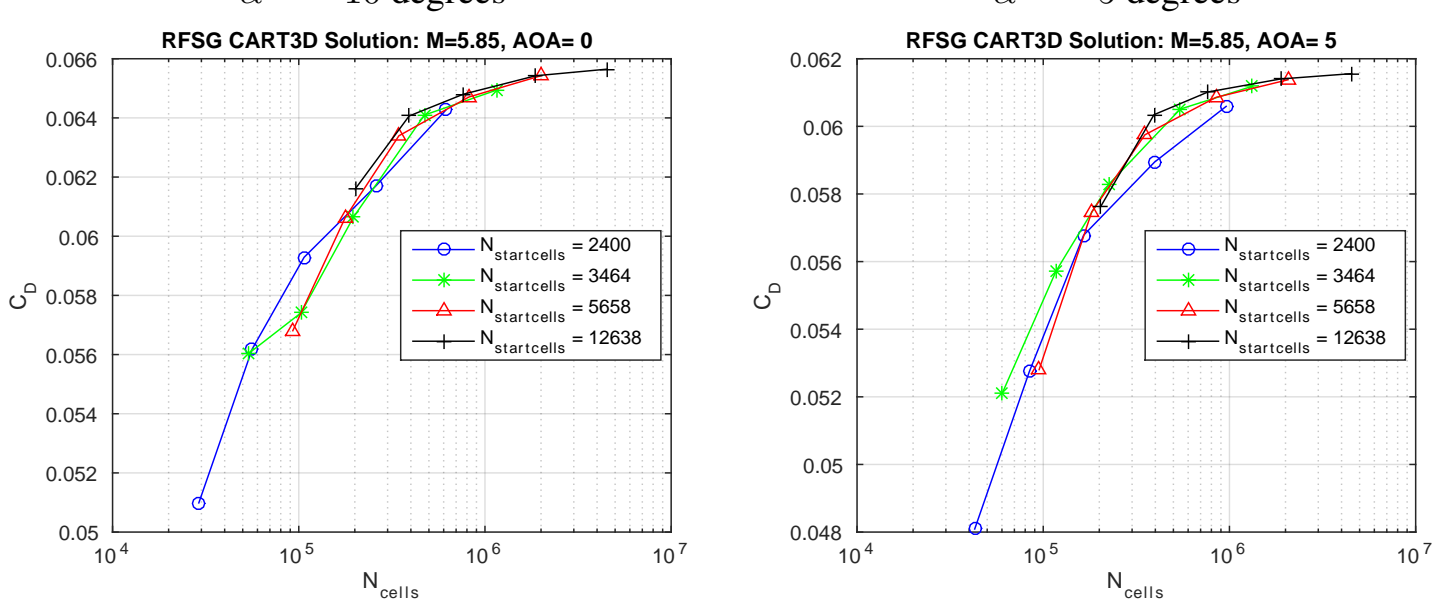

$\alpha=0$ degrees

$\alpha=5$ degrees

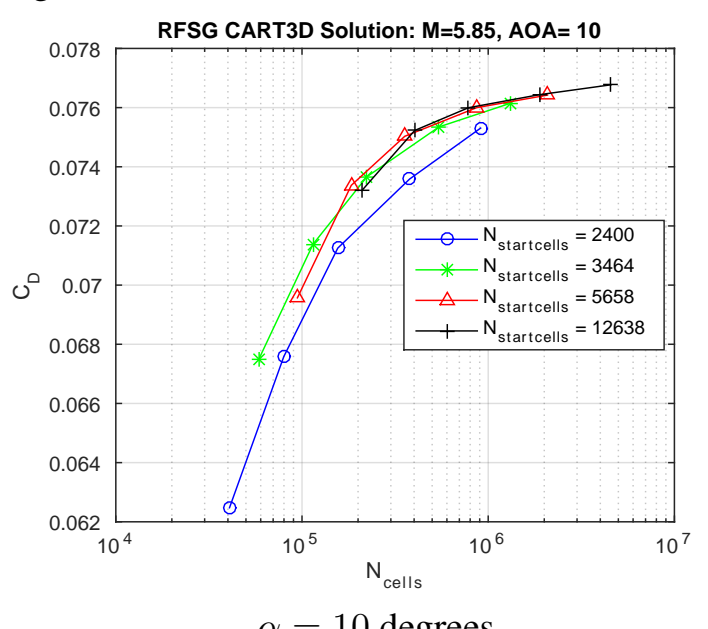

Figure 3.25: CART3D Grid Convergence Study for RFSG geometry for $C_{D}$. 

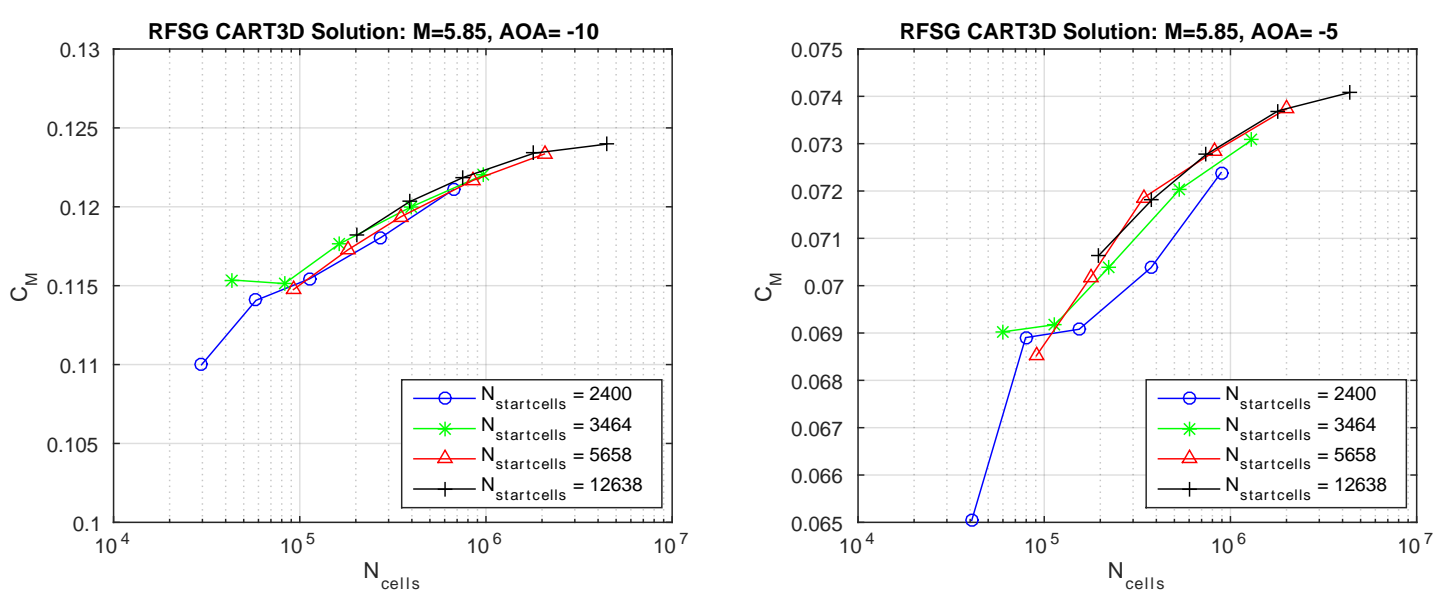

$\alpha=-10$ degrees
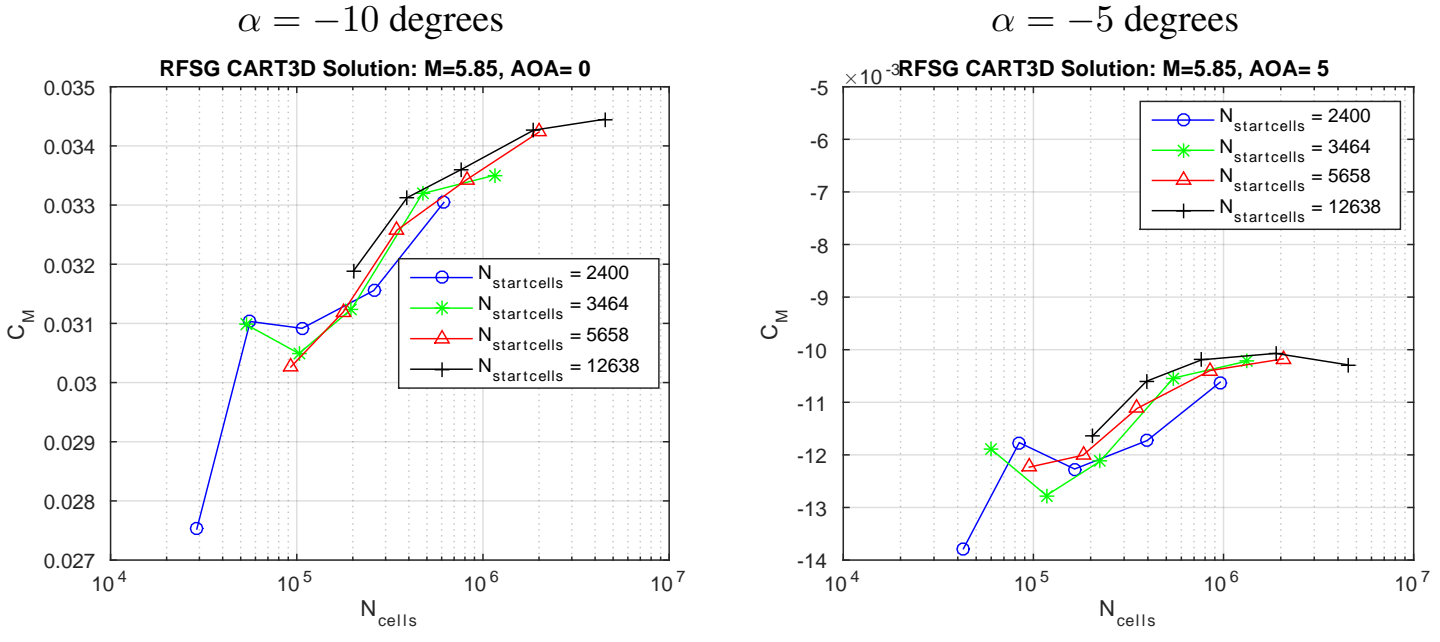

$\alpha=0$ degrees

$\alpha=5$ degrees

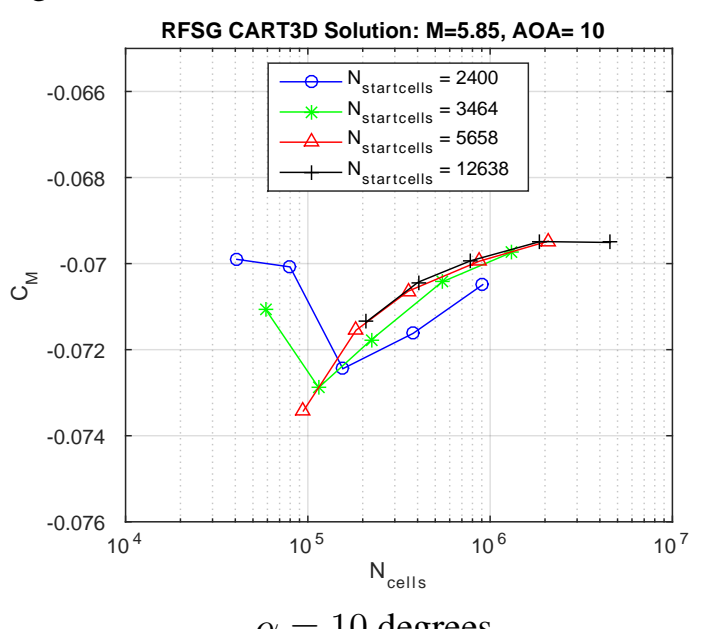

Figure 3.26: CART3D Grid Convergence Study for RFSG geometry for $C_{M}$. 
A comparison between Cart3D and CBAero results is performed next which can give confidence that both analysis codes are producing acceptable results. For comparison, a centerline cut of the RFSG was taken to extract the pressure coefficient value found along the cut for flow conditions at Mach 5.85 and angle-of-attack values of 0,5, and 10 degrees. Very acceptable agreement between upper and lower surface $C_{p}$ distributions from Cart3D and CBAero along the centerline can be seen in Figure 3.27. The cut-plane itself is also shown in the same figure.
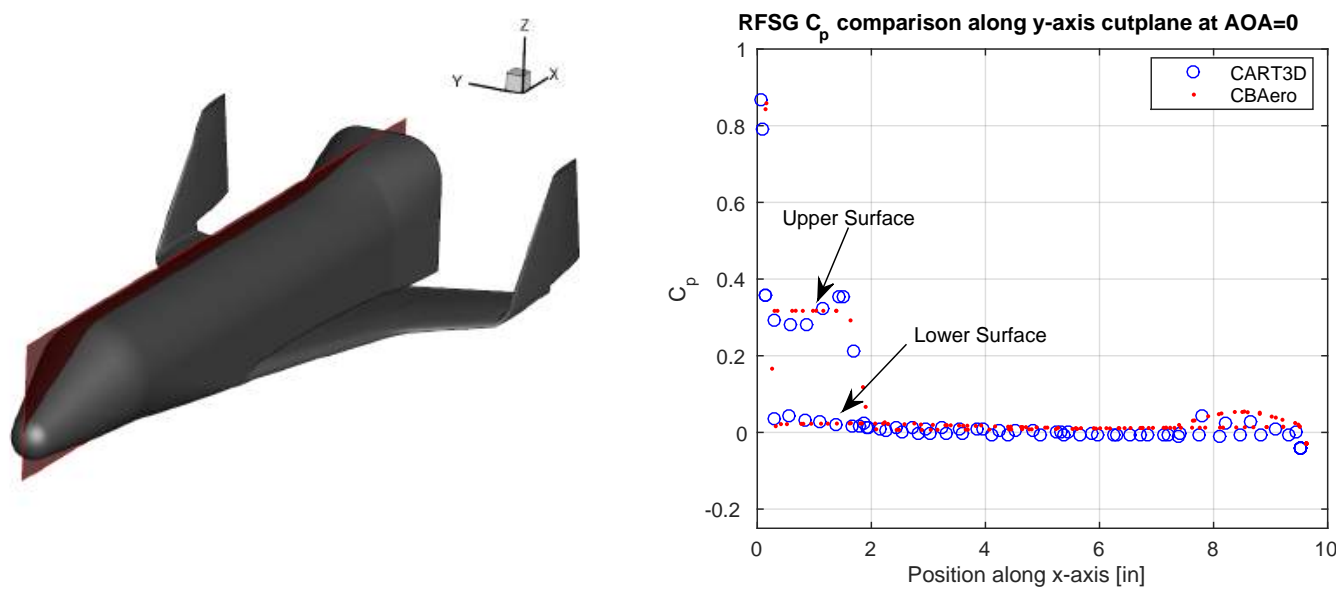

$$
\alpha=0
$$
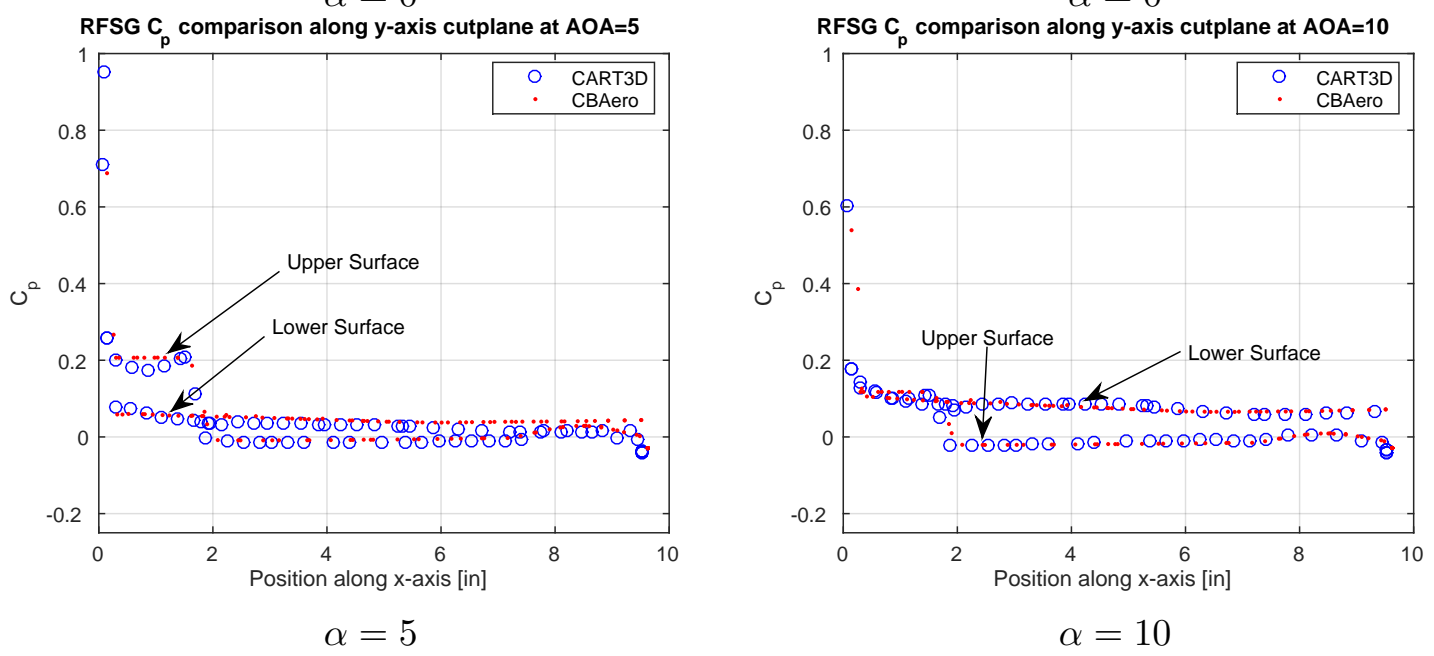

Figure 3.27: CART3D vs. CBAero for RFSG along y-cut plane also shown. 


\subsubsection{GHV}

In a similar fashion to the RFSG, the GHV was analyzed using Cart3D during a grid convergence study. Four initial volume grids were staged through five different adjoint-guided adaptations. Adaptation levels ranged from five to nine adaptations. An input surface mesh of 209, 196 cells was created for input into cubes. This input mesh is provided in Figure 3.28, The residual dropped approximately three to four orders of magnitude. Examples of the mesh adaptation and the residual reduction are again shown for flow conditions at Mach 5.85 and angle-of-attack values of 0,5 , and 10 degrees in Figures 3.293 .30 and Figure 3.31, respectively. Flow features are also illustrated, respectively, with $C_{p}$, Mach, and density contours in Figures 3.32,3.34. Grid convergence results are shown in Figures 3.35 3.37 for $C_{L}, C_{D}$, and $C_{M}$. The final determination from the GHV grid convergence study with Cart3D was that grid-converged integrated quantities are reached, at least, when seven adaptations are used on an initial Cartesian volume grid with cell number greater than roughly $N_{\text {startcells }}=5400$.

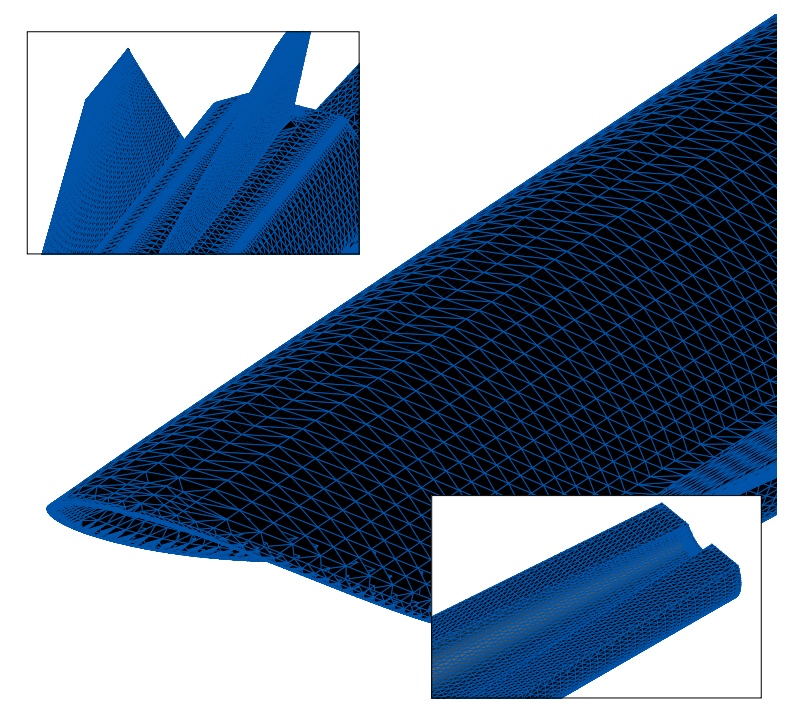

Figure 3.28: GHV Surface Mesh with $N_{\text {cells }}=209,196$. 

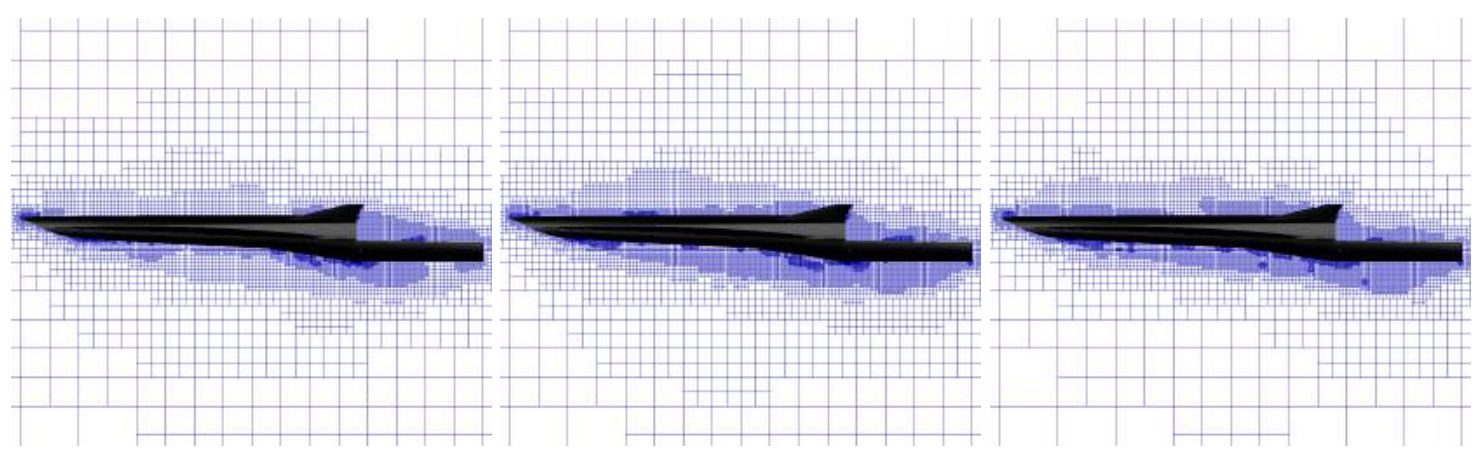

$\alpha=0, N_{\text {cells }}=588,407$

$\alpha=5, N_{\text {cells }}=920,597$

$\alpha=10, N_{\text {cells }}=788,754$

Figure 3.29: GHV Volume Meshes through $y$-cut plane.

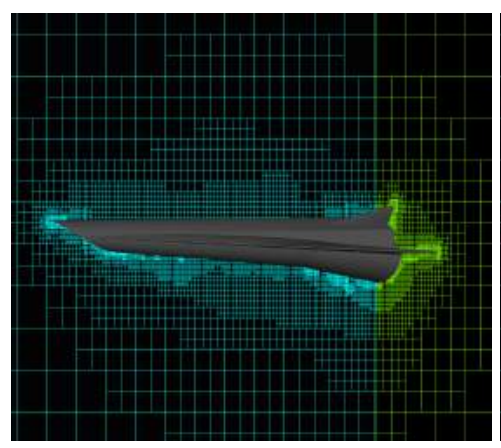

$\alpha=0, N_{\text {cells }}=588,407$

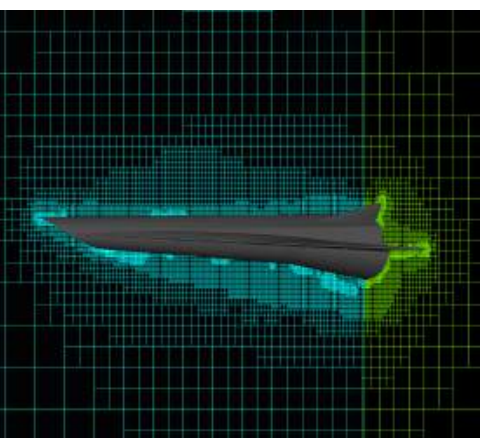

$\alpha=5, N_{\text {cells }}=920,597$

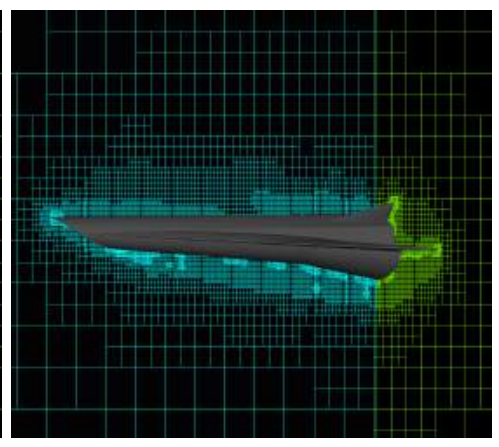

$\alpha=10, N_{\text {cells }}=788,754$

Figure 3.30: GHV Volume Meshes through $x$ and $y$-cut plane. 


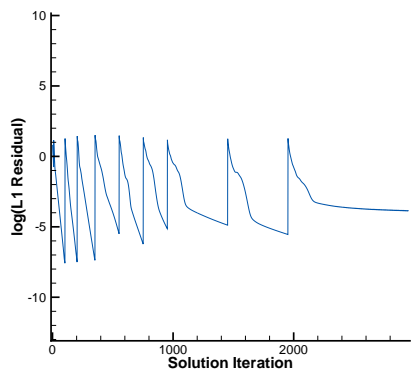

$M=6, \alpha=0$ degrees

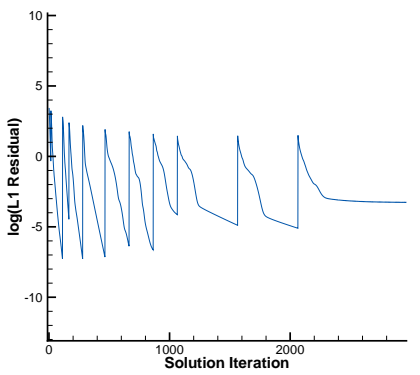

$M=6, \alpha=5$ degrees

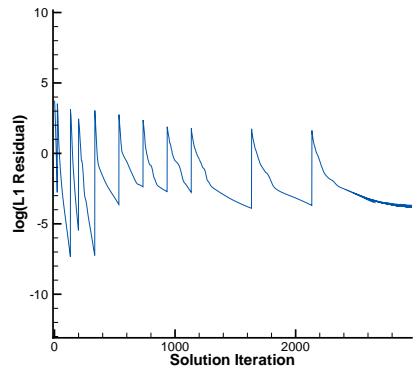

$M=6, \alpha=10$ degrees

Figure 3.31: GHV convergence histories after 8 adaptations.
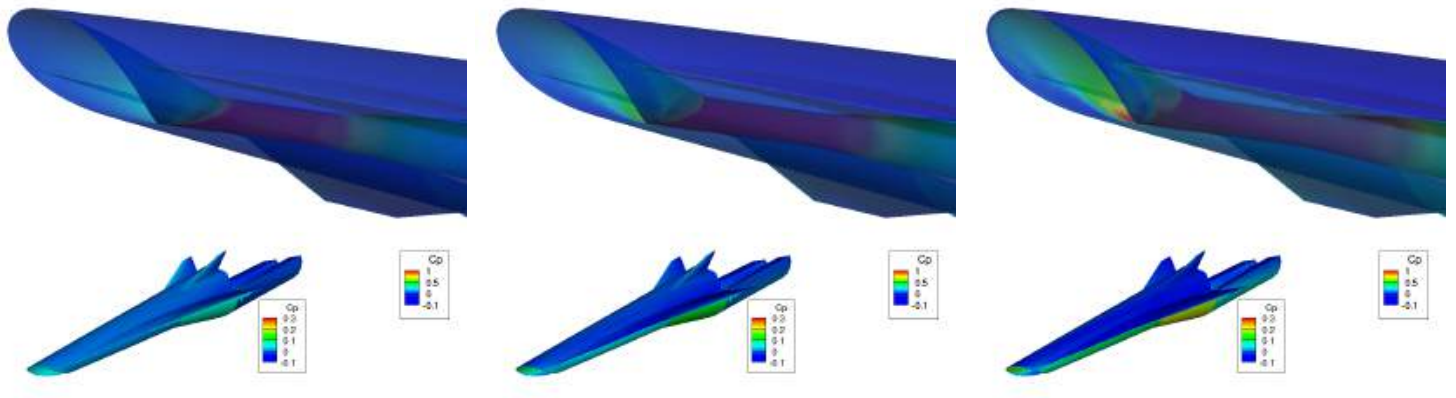

$M=6, \alpha=0$ degrees

$$
M=6, \alpha=5 \text { degrees }
$$

$M=6, \alpha=10$ degrees

Figure 3.32: GHV $C_{p}$ contours. 


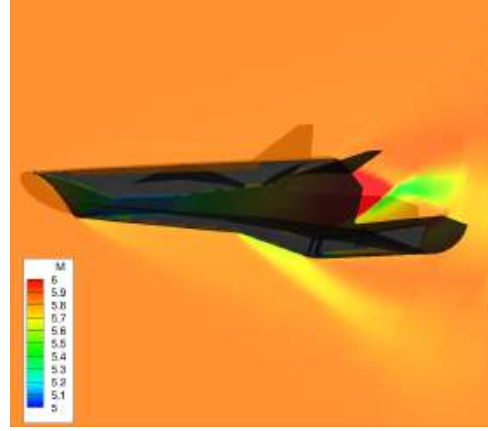

$M=6, \alpha=0$ degrees

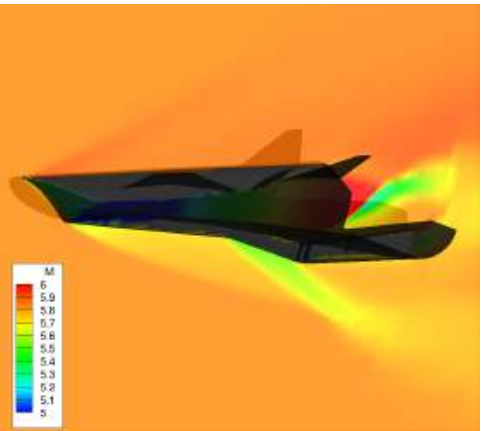

$M=6, \alpha=5$ degrees

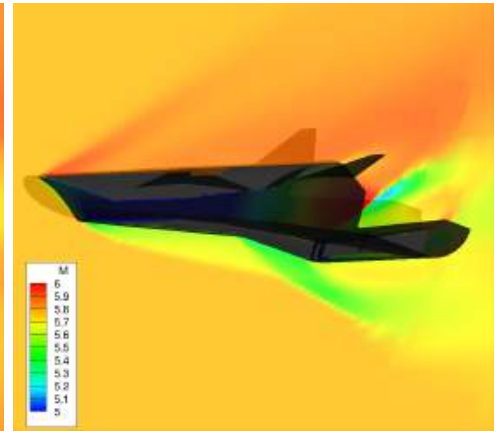

$M=6, \alpha=10$ degrees

Figure 3.33: GHV Mach contours.

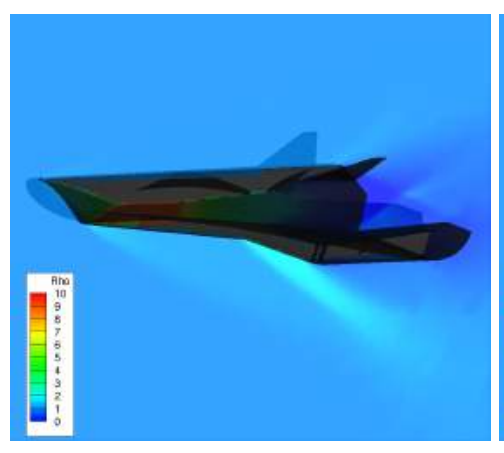

$M=6, \alpha=0$ degrees

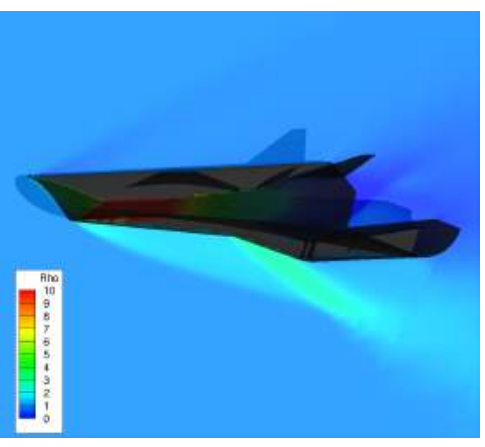

$M=6, \alpha=5$ degrees

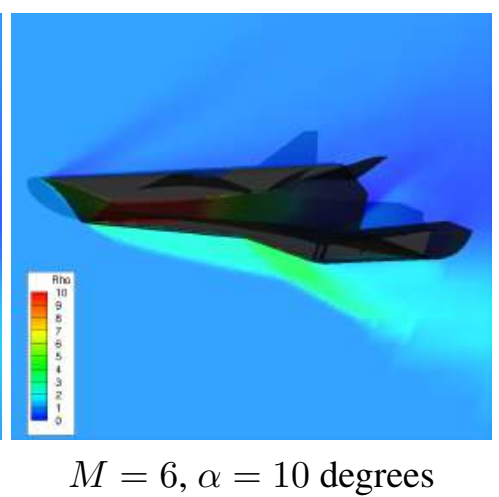

$M=6, \alpha=10$ degrees

Figure 3.34: GHV $\rho$ contours. 

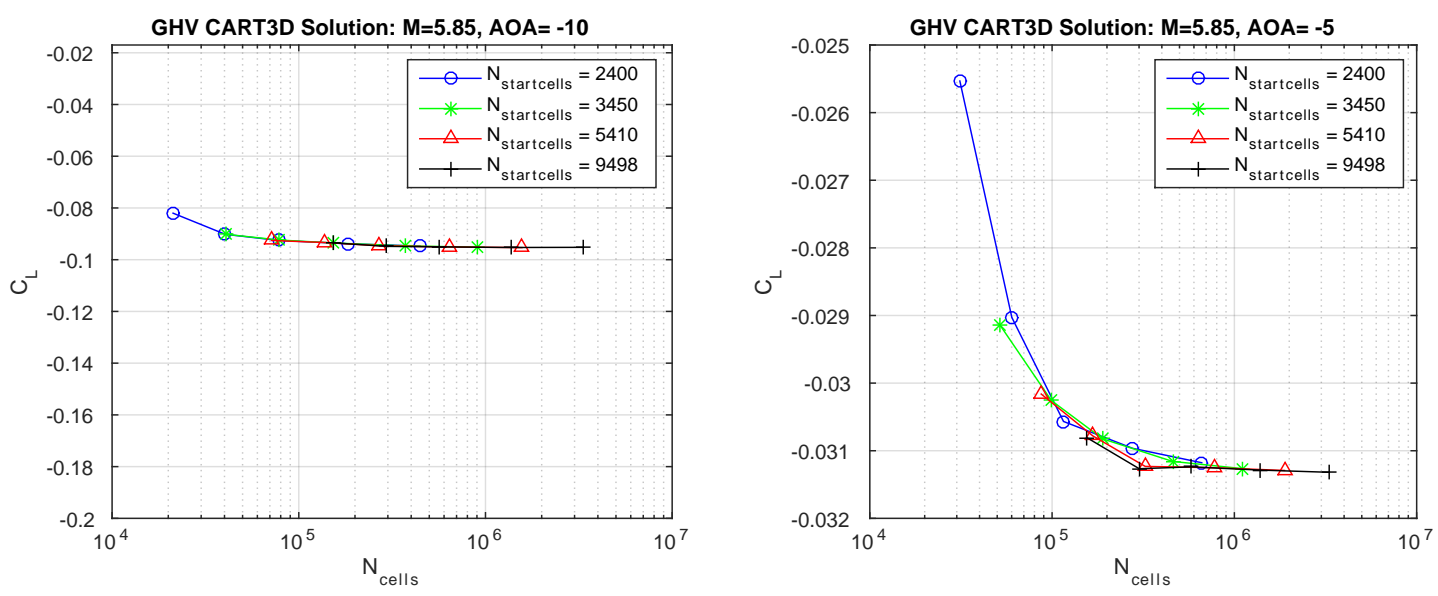

$\alpha=-10$ degrees
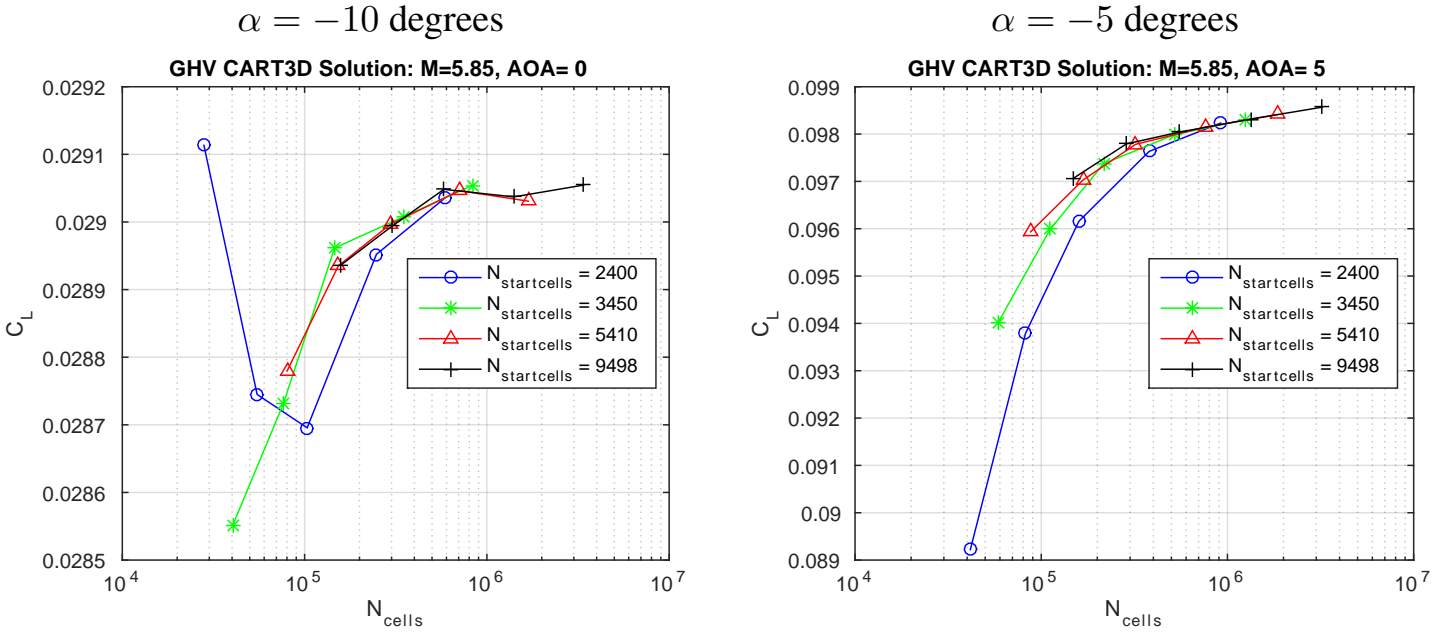

$\alpha=0$ degrees

$\alpha=5$ degrees

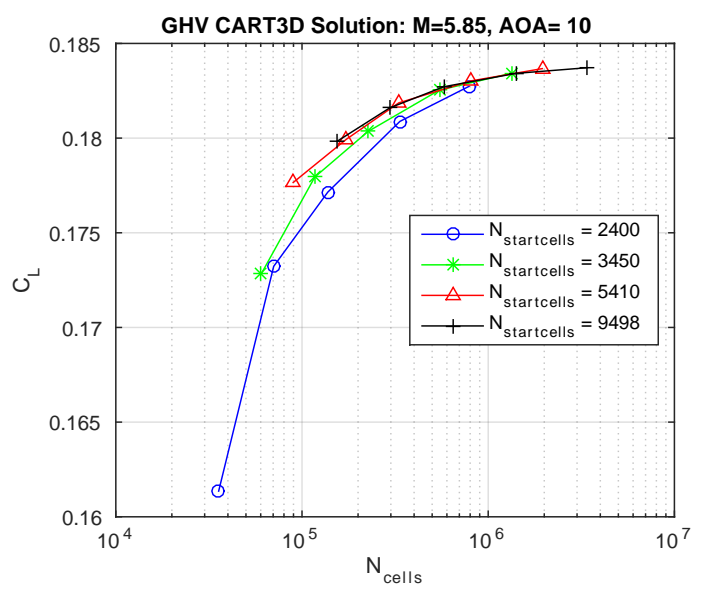

$\alpha=10$ degrees

Figure 3.35: CART3D Grid Convergence Study for GHV geometry for $C_{L}$. 

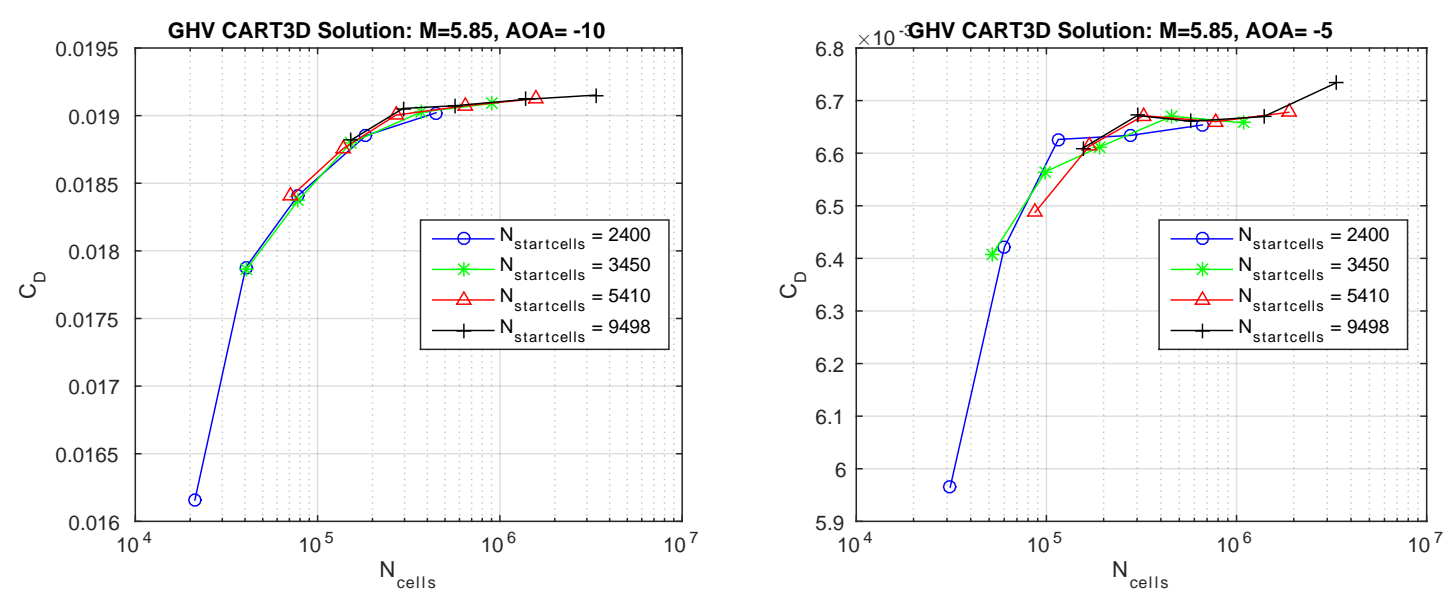

$\alpha=-10$ degrees

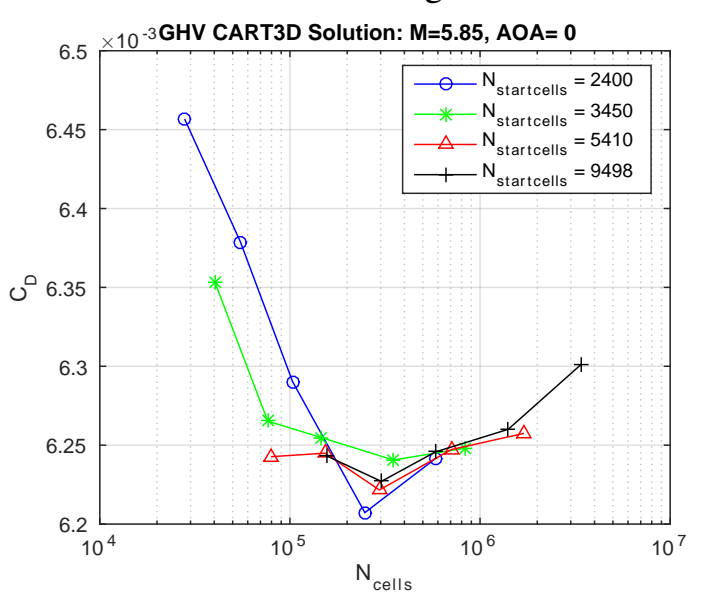

$\alpha=-5$ degrees

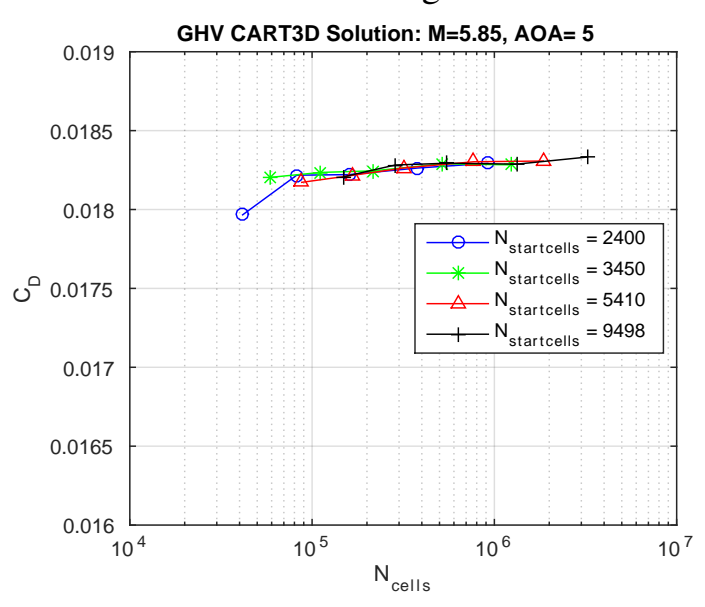

$\alpha=0$ degrees

$\alpha=5$ degrees

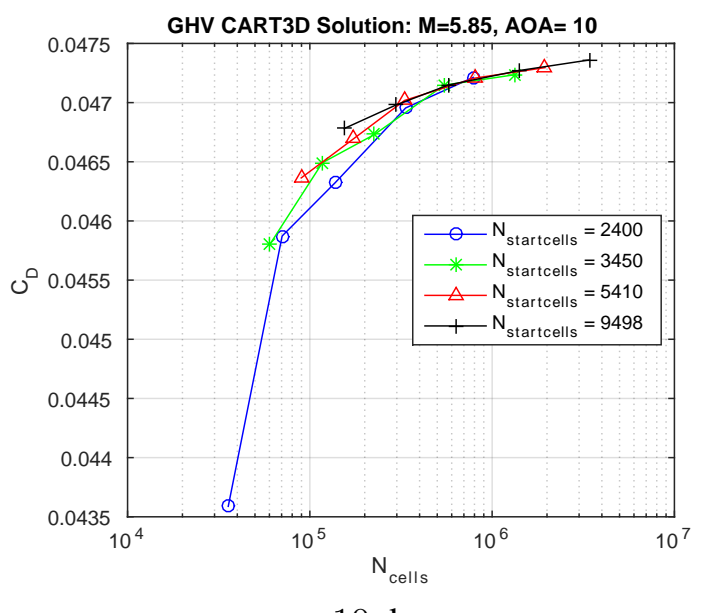

$\alpha=10$ degrees

Figure 3.36: CART3D Grid Convergence Study for GHV geometry for $C_{D}$. 

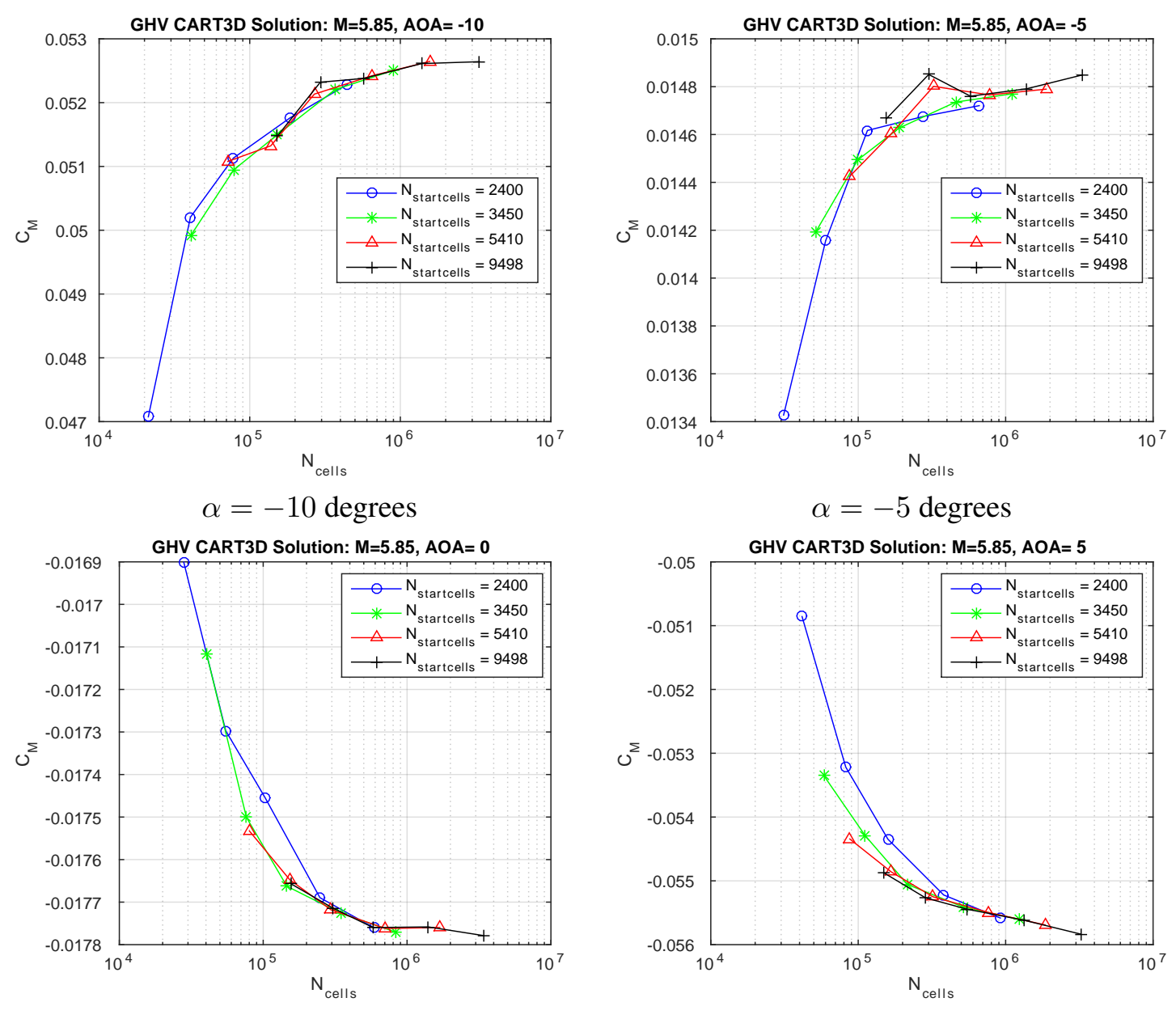

$\alpha=0$ degrees

$\alpha=5$ degrees

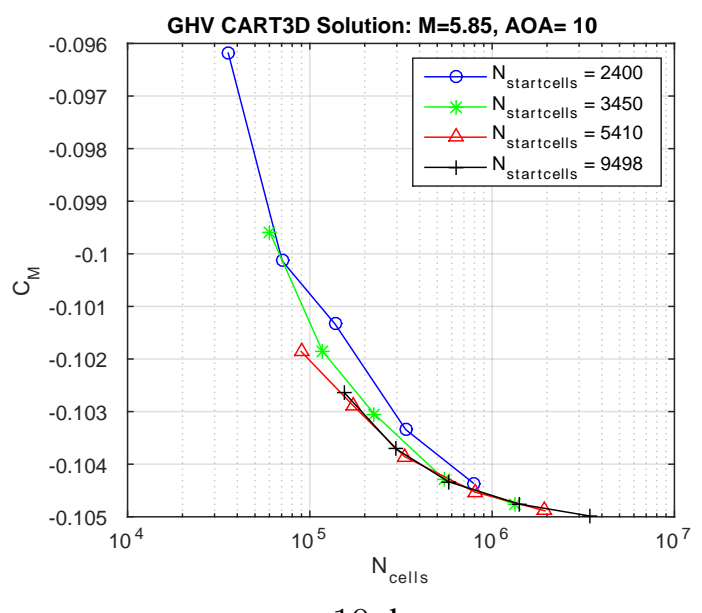

$\alpha=10$ degrees

Figure 3.37: CART3D Grid Convergence Study for GHV geometry for $C_{M}$. 
Again, a comparison is performed between Cart3D and CBAero for a $C_{p}$ distribution along a cut plane of the geometry. A cut was made along the port (left) wing of the GHV, and the $C_{p}$ distribution was extracted. The cut plane can be viewed in Figure 3.38 , and the $C_{p}$ distributions on upper and lower surfaces for 5 and 10 degrees $A O A$ are shown in Figure 3.39 . There is discrepancy between the distributions from both analysis codes. However, trends are quite similar. At these angles-of-attack, the flow can be highly separated, which is not modeled by either code. Cart3D can capture three-dimensional effects, but cannot accurately model separation since separation is a highly viscous phenomenon. CBAero has no ability to model three-dimensional flow effects or even communication of flow from one point to the next. Therefore, even though there is discrepancy in the two distributions, there is still confidence that the proper trends are being resolved by both codes.

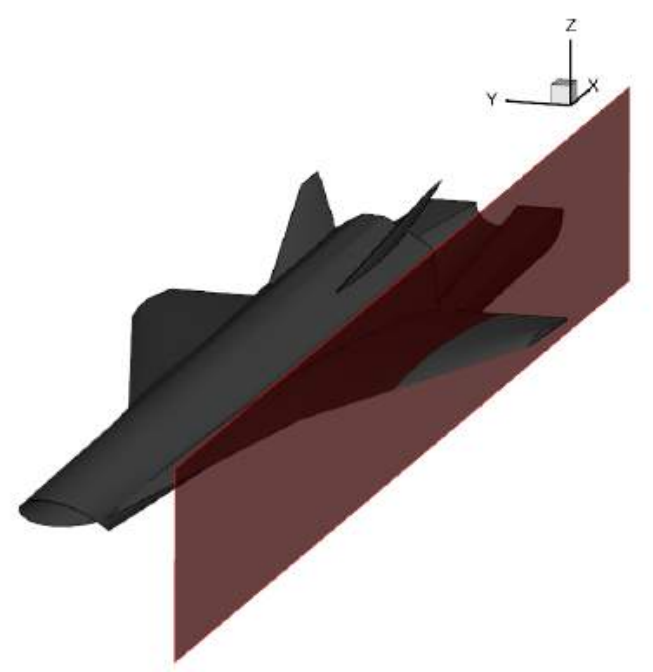

Figure 3.38: GHV cut plane along port side wing. 

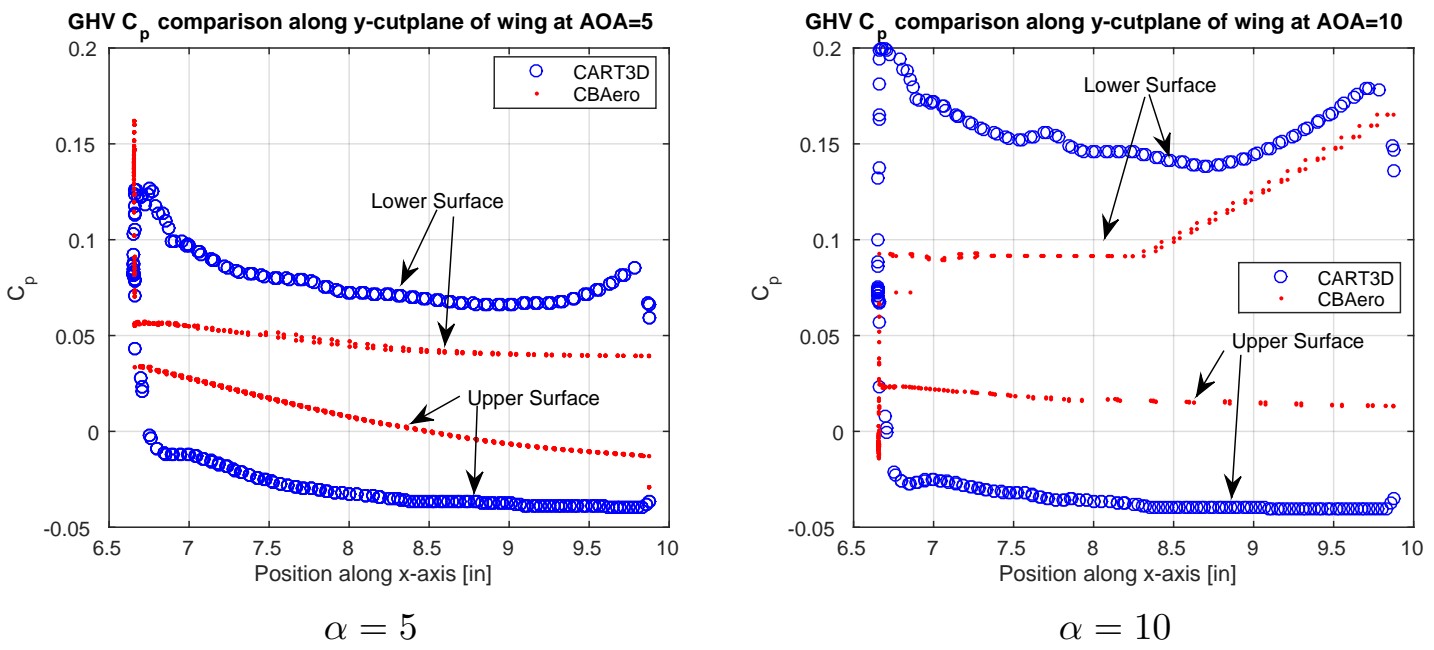

Figure 3.39: CART3D vs. CBAero for GHV along y-cut plane of wing. 


\section{CHAPTER IV}

\section{VISCOUS NUMERICAL ANALYSIS}

The ability to predict convective heating over a three-dimensional body at hypersonic speeds is very important in the early stages of hypersonic vehicle design. These simulations cannot be performed accurately with an inviscid flow assumption and two different approaches utilized in this work to account for viscous effects as well as their results for the HIFiRE-1 geometry are discussed in the next two sections.

\subsection{UNLATCH Boundary Layer Solver}

The UNLATCH (Unstructured Langley Approximate Three-Dimensional Convective Heating) engineering code can be used to calculate the heating rates on general three-dimensional bodies using inviscid flow field results from an unstructured flow solver, such as Cart3D. This is done by reducing the three-dimensional boundary layer equations to the same form as the axisymmetric boundary layer equations [22]. UNLATCH was used in conjunction with the inviscid flow field results from Cart3D, shown in Section 3.2.1, to approximate the surface temperatures on the body of the HIFiRE-1 geometry.

The cases shown in Table 4.1 were run using UNLATCH in order to approximate the viscous effects of the boundary layer on the body with a constant wall temperature of $300 \mathrm{~K}$. These are the same conditions as the ones used during the wind tunnel experiments except for the constant wall temperature. 
Table 4.1: UNLATCH Run Conditions.

\begin{tabular}{c|c|c|c|c} 
& Case 1 & Case 2 & Case 3 & Case 4 \\
\hline \hline$V_{\infty}\left[\frac{\mathrm{m}}{\mathrm{s}}\right]$ & 936 & 986 & 936 & 986 \\
\hline$p_{\infty}\left[\frac{\mathrm{kg}}{\mathrm{m}^{3}}\right]$ & 3570 & 3570 & 10200 & 10200 \\
\hline$T_{\infty}[\mathrm{K}]$ & 63.7 & 70.8 & 63.7 & 70.8
\end{tabular}

\subsubsection{Meshing Strategy for UNLATCH}

There was considerable difficulty in reaching flow solutions from Cart3D that could be successfully input into UNLATCH. Stagnation regions and areas of high flow interaction must be resolved to a significant degree in order for UNLATCH to begin its viscous approximation. As a result, a very large number of trades in Cart3D adaptation strategy were made in order to find a combination of adaptation and resolution that could be acceptable for UNLATCH. Due to this difficulty, only solutions for the HIFiRE-1 geometry were successfully input into UNLATCH at this point.

In order to sufficiently resolve the flow solution about the HIFiRE-1 payload geometry, a different Cart3D adaptation strategy had to be employed. The stagnation region near the nose of the geometry had to be discretized with enough resolution such that the resulting solution on the surface was relatively smooth, as shown in Figure 4.1. To do this, the nose itself was identified as a separate component from the body. When the initial volume mesh was generated, refinement of the grid was denoted to take place at the nose, allowing the adjoint refinement of the mesh to identify the nose specifically as a location to cluster cells.

The advantage of using the robust Cart3d Euler solver is in the ability for the solver to cluster cells where the user defined flow functional degrades the overall quality in specific areas within the off body mesh. This ability allows for Cart3d to build the volume mesh without significant input by the user, which leads to a significant savings of time due to rapid mesh generation. When this 
advantage is taken away, in the case of pairing UNLATCH, the main benefit of using Cart3d is not as apparent and suggests going to a higher fidelity RANS solution if significant user input is needed for mesh generation.

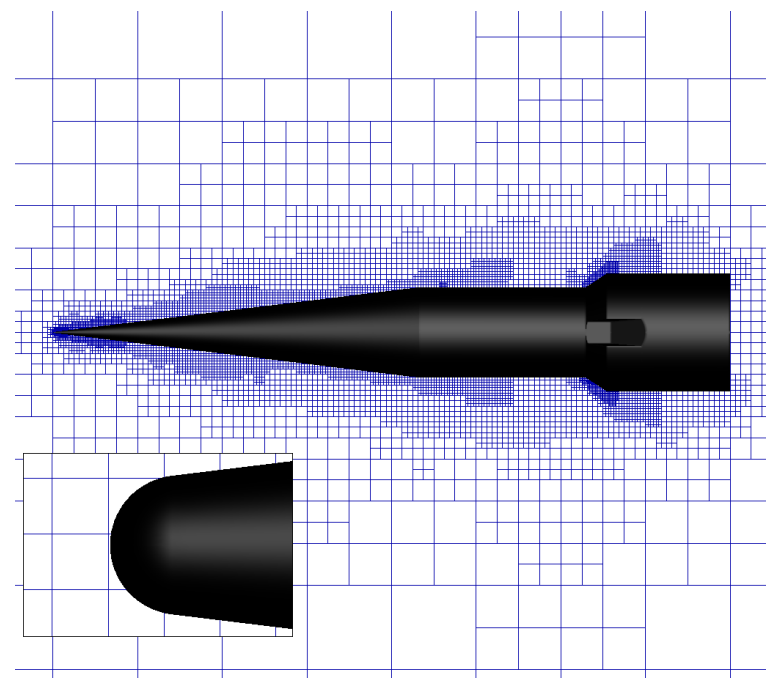

Original clustering for Cart3d

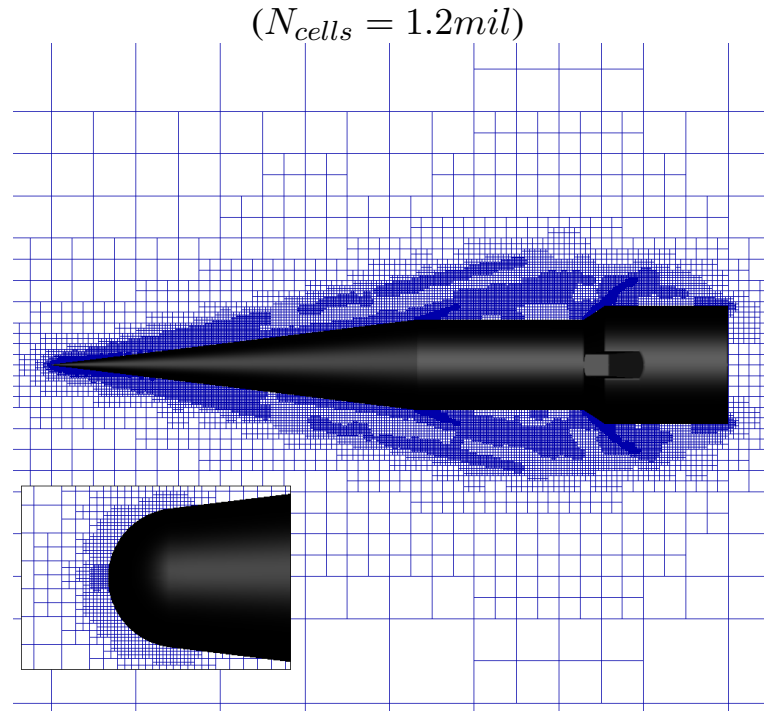

Clustering needed for UNLATCH

$\left(N_{\text {cells }}=6.4 \mathrm{mil}\right)$

Figure 4.1: HIFiRE-1 volume mesh for Cart3d case vs. UNLATCH case at $A O A=0$ degrees 


\subsubsection{UNLATCH Results for HIFiRE-1}

Some of the employed surface and volume meshes have already been shown previously in Figures 3.11 to 3.13 . Pertinent flow features are showcased in Figures 4.24 .4

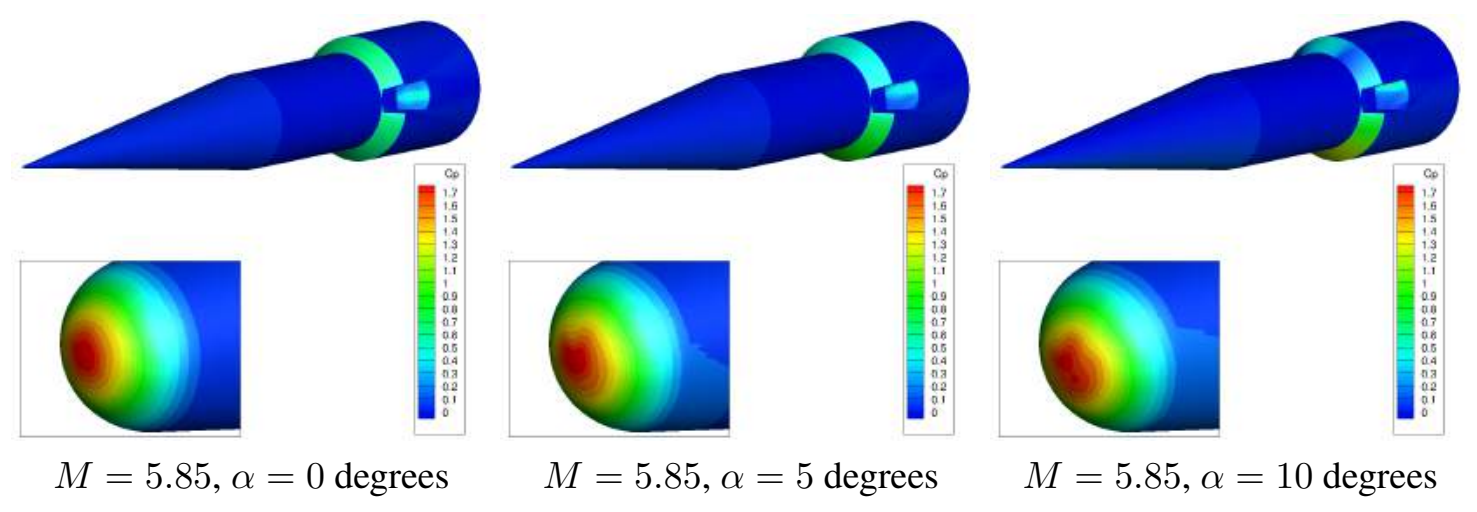

Figure 4.2: CART3D plus UNLATCH $C_{p}$ contours for HIFiRE-1 geometry.

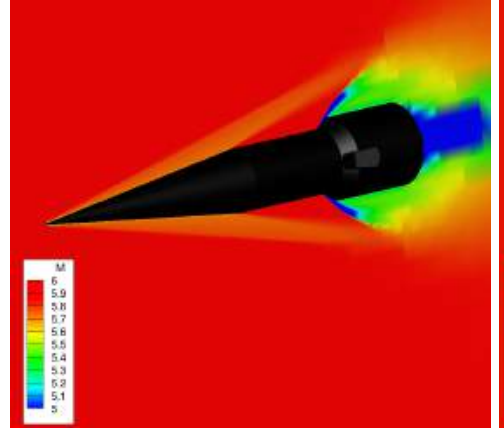

$M=5.85, \alpha=0$ degrees

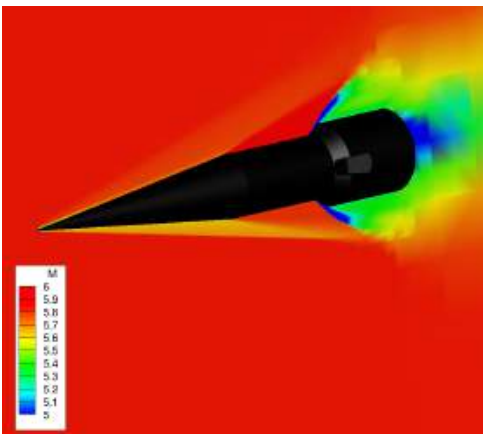

$M=5.85, \alpha=5$ degrees

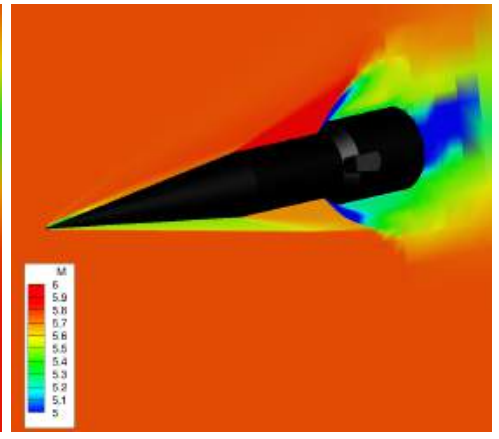

$M=5.85, \alpha=10$ degrees

Figure 4.3: CART3D plus UNLATCH Mach contours for HIFiRE-1 geometry. 


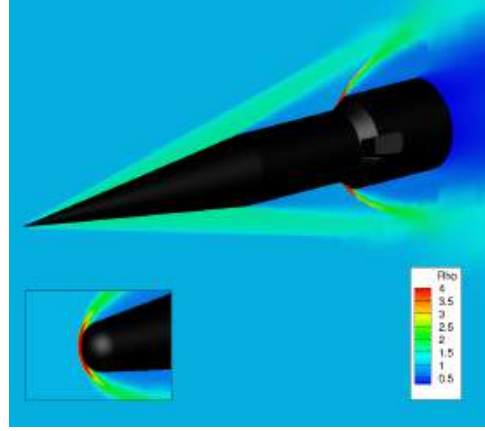

$M=5.85, \alpha=0$ degrees

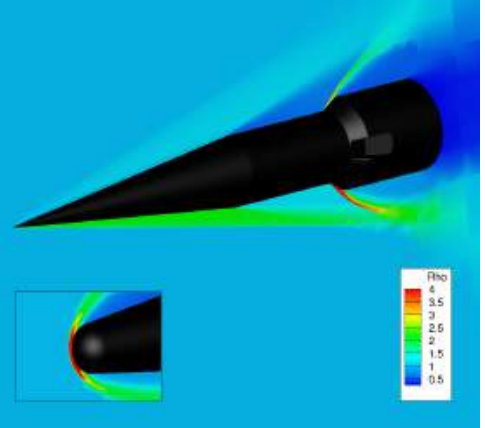

$M=5.85, \alpha=5$ degrees

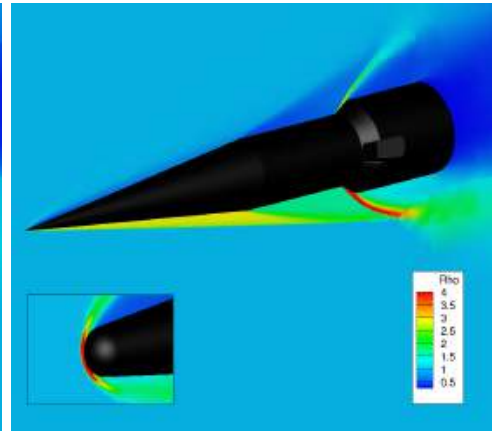

$M=5.85, \alpha=10$ degrees

Figure 4.4: CART3D $\rho$ contours for HIFiRE-1 geometry [6]

The heating rate contours predicted by UNLATCH for the HIFiRE-1 geometry are shown in Figure 4.5. Unfortunately, due to a bug in the UNLATCH source code, output of surface wall temperatures from UNLATCH is currently unavailable. 

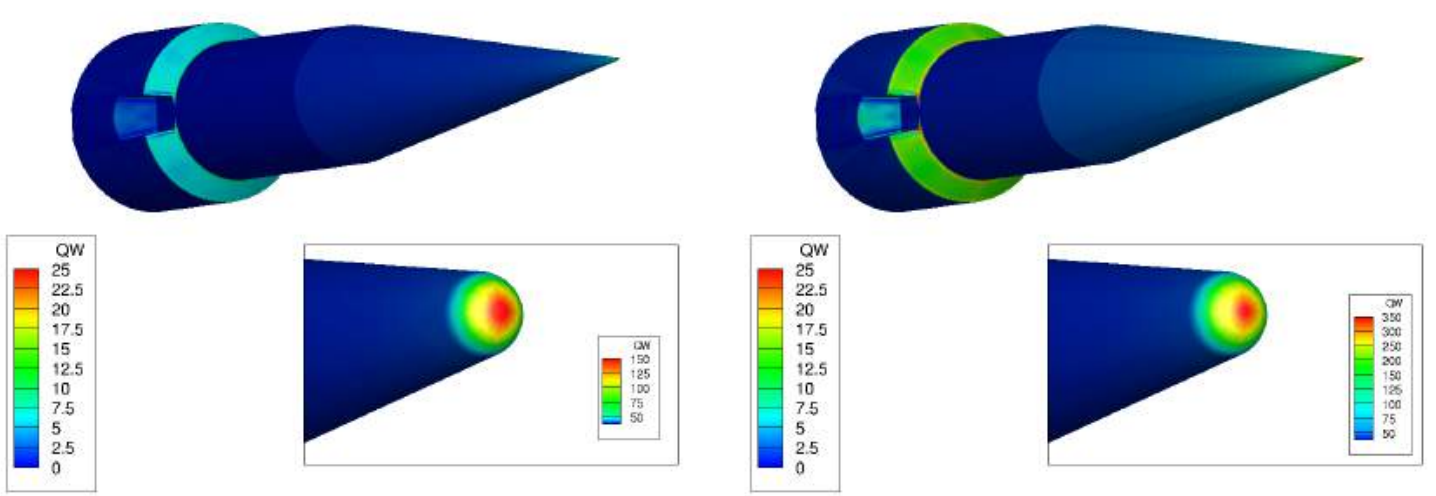

Case $1, \alpha=0$ degrees

Case $4, \alpha=0$ degrees
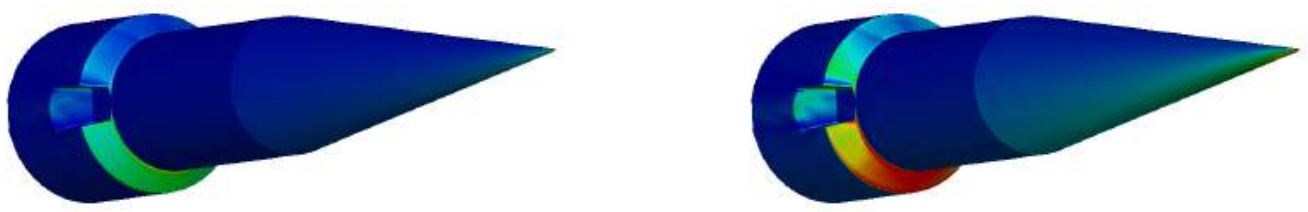

\begin{tabular}{|l|}
\hline $\mathrm{QW}$ \\
25 \\
22.5 \\
22.5 \\
20 \\
17.5 \\
15 \\
12.5 \\
10 \\
7.5 \\
5 \\
2.5 \\
0 \\
\hline
\end{tabular}
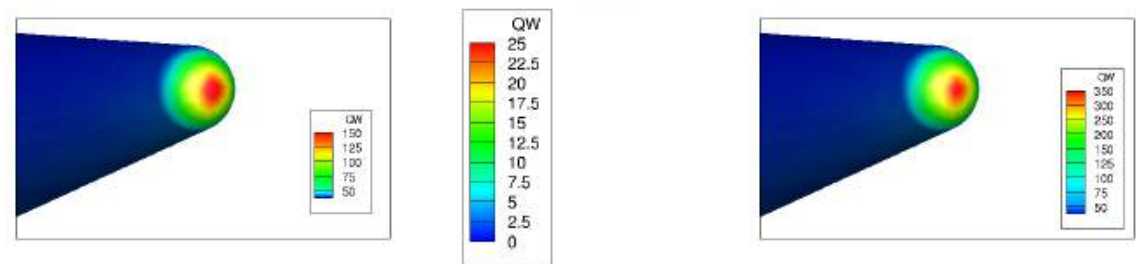

Case $1, \alpha=10$ degrees

Case $4, \alpha=10$ degrees

Figure 4.5: UNLATCH solution for surface heating rates $\left[\frac{W}{m^{2}}\right]$ for HIFiRE-1 Geometry.

\subsection{FUN3D RANS Solver}

FUN3D is an unstructured finite-volume viscous flow solver [23]. The choice to use Fun3d as the viscous solver for this research was due to availability, being a NASA supported code, and ease 
of use on the High Performance Computing Center here at Wright-Patterson Air Force Base. For the HIFiRE-1 geometry, three unstructured meshes were made using Numeca Hexpress, and the finest mesh is shown in Figure 4.6. The free stream conditions from Case 1 in Table 4.1 were used to match the experimental testing of the model leading to a Reynolds number of roughly $43 \mathrm{million} / \mathrm{m}$.

\subsubsection{Mesh Generation}

The volume grid for the HIFiRE-1 geometry to be used in Fun3d was generated using Numeca HEXPRESS/Hybrid [20] and Numeca HEXPRESS/View [24]. Numeca Hexpress is a fully automatic hexahedral dominant grid generator that can generate both surface and volume meshes for 2D and 3D geometries [25]. The decision to use Numeca, instead of a manual griding tool such as Pointwise or its predecessor GridGen [19] as used in previous analysis was to evaluate the tool in terms of how well it could build a viscous volume mesh for high fidelity numerical analysis.

The process for generating a volume grid in Numeca is as follows: 


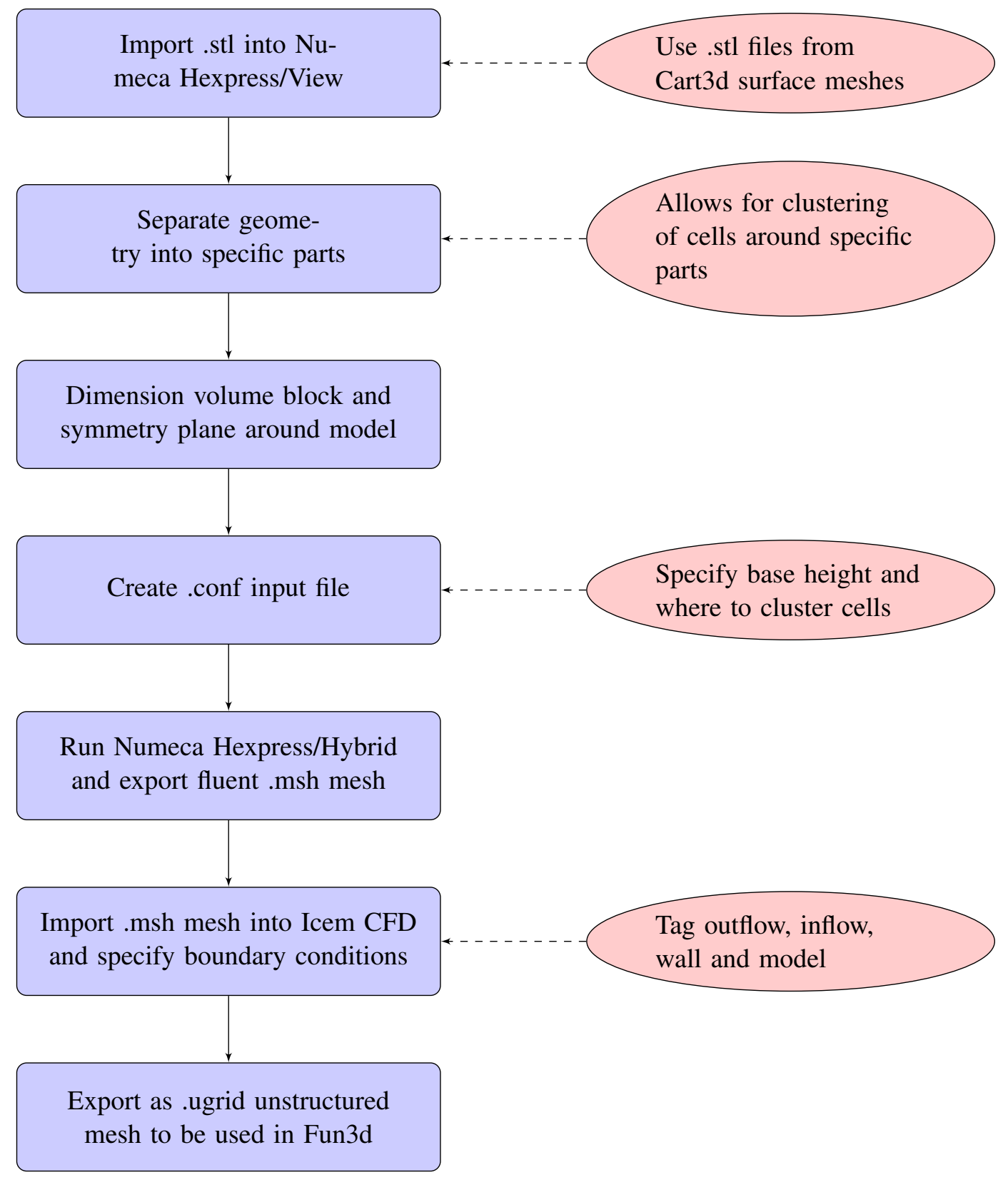


The input .conf file for Numeca Hexpress/Hybrid [20] is shown in its entirety in Appendix B for the fine HIFiRE-1 volume mesh ( 21 mil cells). The HIFiRE-1 model (HF1initial.spb) and the farfield components (box.spb), comprised of the farfield, outflow, and symmetry plane, were exported and created using Numeca Hexpress/View [24]. The interesting components of the .conf are shown and explained below:

Table 4.2: Numeca .conf file components

\begin{tabular}{c|c|c} 
Term & Value & Explanation \\
\hline \hline WRAPPING & - & When enabled, this created a surface mesh only \\
\hline BASEH & 1.4 & Numeca internal setting that specifies overall refinement of mesh \\
\hline PROXIMITYGROUP & - & Defines increased refinement where specified parts intersect
\end{tabular}

The boundary condition specifications for the HIFiRE-1 mesh within Fun3d are shown below in Table 4.3 These boundaries, as constructed within Numeca Hexpress, are then re-tagged with a re-connection flag using Icem CFD [26]. This results in a .fbc files being exported from Icem CFD and then used to create the mapbc file shown below.

Table 4.3: HF1 boundary conditions from .mapbc file

\begin{tabular}{c|c|c} 
BC input for Fun3d & Name from Icem CFD .ugrid mesh & Type of BC \\
\hline \hline 5000 & Farfield & Riemann invariants \\
\hline 4000 & Model & $\begin{array}{c}\text { Viscous explicitly } \\
\text { set the no-slip condition }\end{array}$ \\
\hline 6022 & SYMplane & $\begin{array}{c}\text { Symmetry enforced replacing } \\
\text { y-momentum with zero normal velocity }\end{array}$ \\
\hline 5026 & Outflow & $\begin{array}{c}\text { Extrapolate supersonic outflow } \\
\text { specified via fluxes }\end{array}$
\end{tabular}


When a mesh is created in Numeca, the following list of mesh criteria statistics is printed, shown in Table 4.4. The main flags to look for in this is to ensure that there are no negative tets, Jacobian's or concave or twisted cells.

Table 4.4: Numeca quality criteria for HIFiRE-1 viscous volume mesh

\begin{tabular}{c|c} 
Criteria & Value \\
\hline \hline Negative part tets & 0 \\
\hline Negative Jacobian & 0 \\
\hline Concave cells & 0 \\
\hline Twisted cells & 0 \\
\hline Max ratio 1.0 & 0.99 \\
\hline Max ratio $>0.95$ & $4,161,928$ \\
\hline Max ratio $>0.9$ & $5,873,187$ \\
\hline Min edge length & $1.99 \mathrm{e}-05$ \\
\hline Average edge length & 0.00345 \\
\hline Max edge length & 1.30136 \\
\hline Max equiangular skewness & 0.974376 \\
\hline Max adjacent volume ratio & 23.5911 \\
\hline Max expansion ratio & 13.7857
\end{tabular}

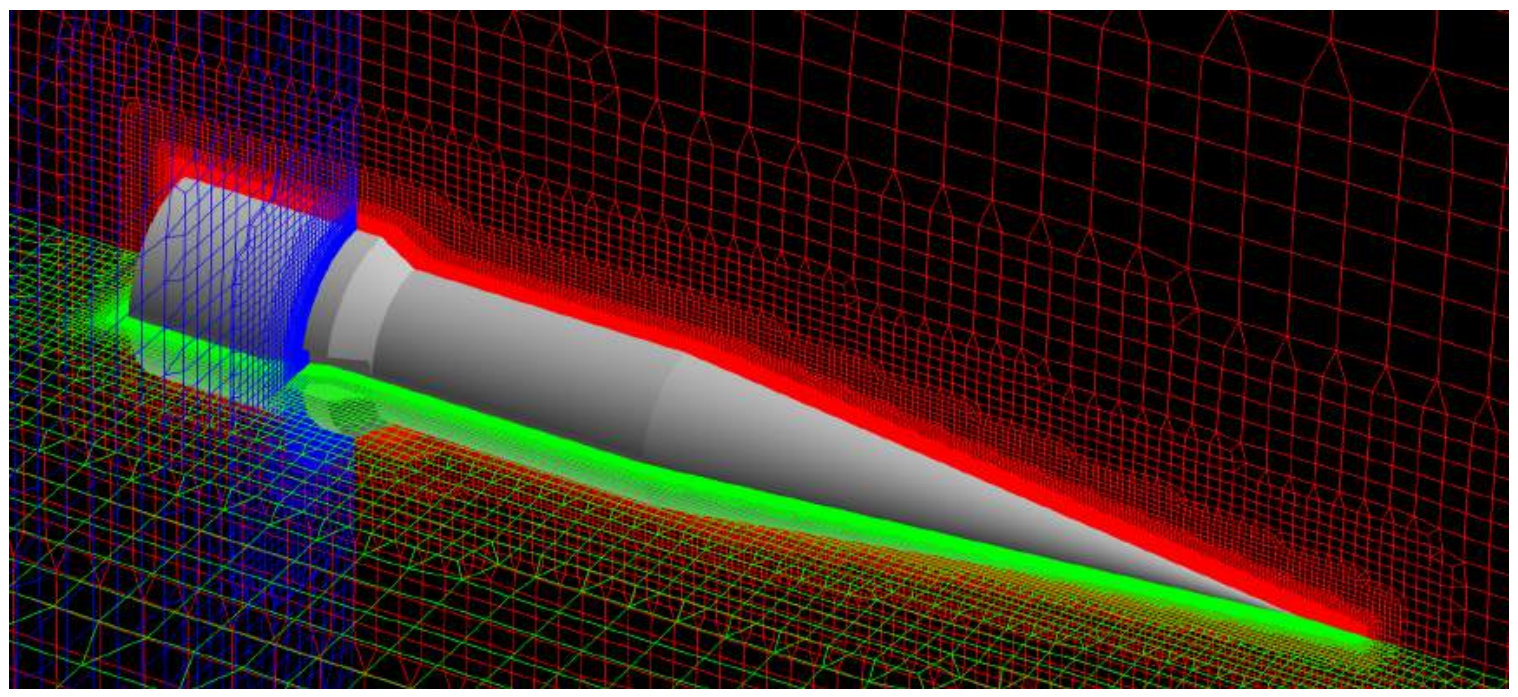

Figure 4.6: HIFiRE-1 volume grid for FUN3D, Fine Mesh, $N_{\text {cells }}=21,815,736$. 
No mesh adaptation was used for the various angles of attack. The increasing clustering of cells in the boundary layer of the various meshes can be seen in Figure 4.7.

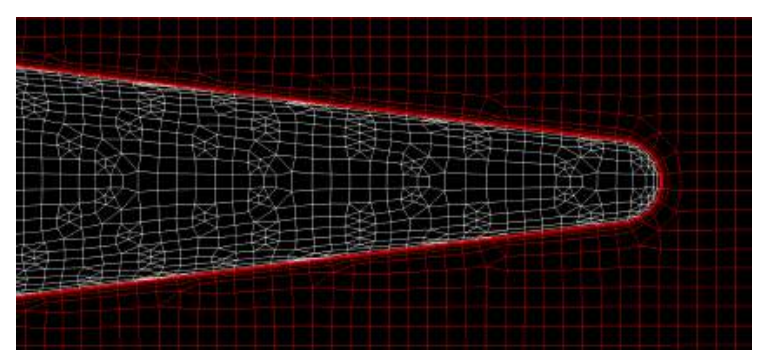

Coarse, $N_{\text {cells }}=3,355,960$

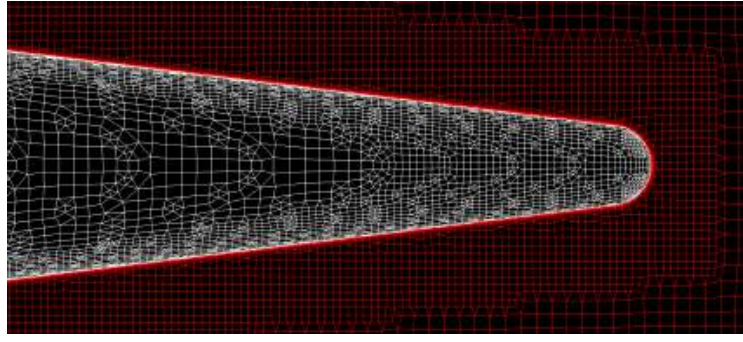

Medium, $N_{\text {cells }}=12,262,968$

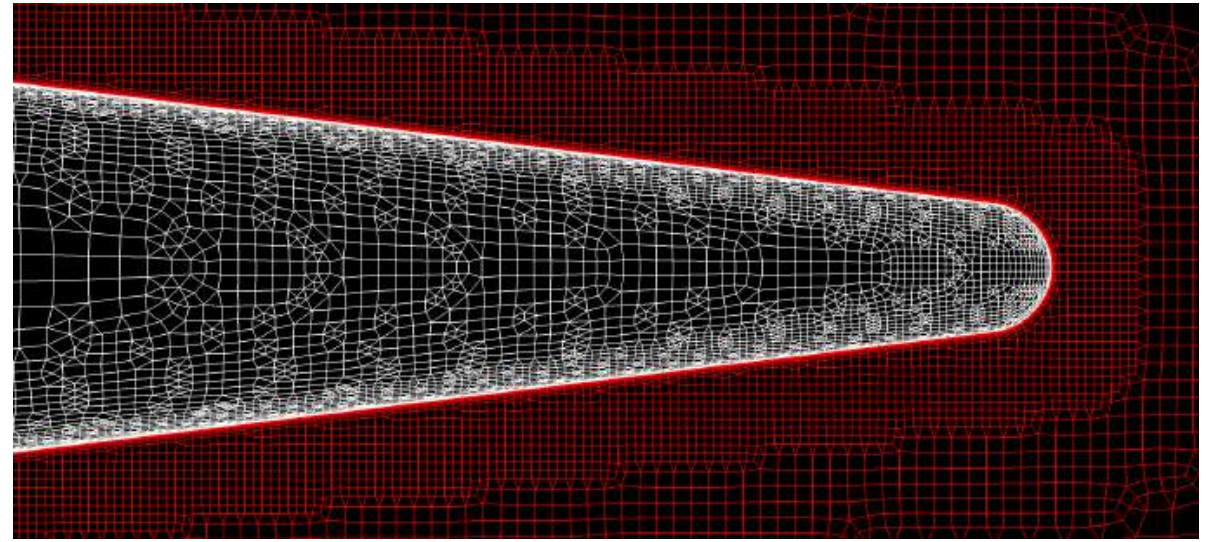

Fine, $N_{\text {cells }}=21,815,736$

Figure 4.7: HIFiRE-1 volume grid for FUN3D around nose.

\subsubsection{Input Parameters}

The Fun3d input (fun3d.nml) file that was used to run the HIFiRE-1 fine mesh can be found in Appendix C, for the first restart, shown in Figure 4.6 at $\alpha=5$ degrees and $M=5.85$. In order to initialize the flow, a cylinder was built around the HIFiRE-1 geometry to begin at a lower subsonic Mach number starting within the boundary layer to allow for the flow to slowly progress from zerovelocity at the no-slip boundary to the free stream Mach 6 flow. Due to the mesh from Numeca 
being in inches, a scaling factor of 0.0254 was used to convert the mesh to meters, which is what Fun3d requires.

A Reynolds number of $\sim 44$ mil was used for each of the Fun3d runs, which is non-dimensionalized to be Reynolds number per meter in Fun3d. The solution was progressed from turbulent unsteady to turbulent steady in the final restart. After the final steady state solution was reached, 0 and 10 degree AOA were ran.

In order to get to the final, steady state solution, four restarts were required with the CFL number being varied for each in order to allow the equations to be solved. Each restart progressed the solution, starting with the solution from the previous run. The progression of the CFL number and number of steps for each restart is shown in Table 4.5 .

Table 4.5: Restarts for HIFiRE-1 in Fun3d

\begin{tabular}{|c|c|c|c|c|}
\hline Restart & $\begin{array}{l}\text { Equation Type } \\
\text { Viscous Terms }\end{array}$ & Time Accuracy & Steps & CFL Number \\
\hline 1 & $\begin{array}{c}\text { Compressible } \\
\text { Turbulent }\end{array}$ & 2nd Order & 30,000 & $2.5 \mathrm{e}-6 \rightarrow 0.70$ \\
\hline 2 & $\begin{array}{c}\text { Compressible } \\
\text { Turbulent }\end{array}$ & 2nd Order & 80,000 & $0.07 \rightarrow 0.70$ \\
\hline 3 & $\begin{array}{c}\text { Compressible } \\
\text { Turbulent }\end{array}$ & 2nd Order & 50 & $0.07 \rightarrow 2.0$ \\
\hline 4 & $\begin{array}{c}\text { Compressible } \\
\text { Turbulent }\end{array}$ & Steady & 30,000 & $0.07 \rightarrow 2.0$ \\
\hline
\end{tabular}

\subsubsection{HIFiRE-1 Results}

A typical convergence history of mass, momentum, energy and turbulence working variable for the Spalart-Allmaras model exhibiting good convergence is shown in Figure 4.8. Each jump in residual shown is a result of a restart from the previous solution. 


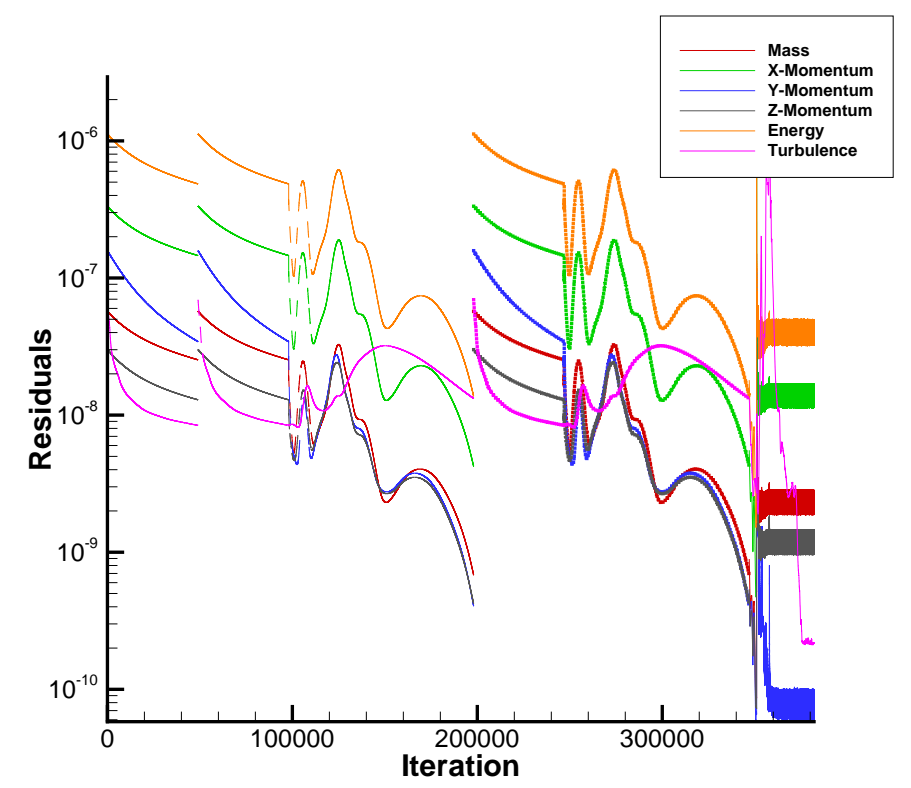

Figure 4.8: HIFiRE-1 FUN3D Convergence History for fine mesh.

The integrated quantities of $C_{L}$ and $C_{D}$ for the various meshes are plotted in Figure 4.9 showing grid convergence. Thus, the mesh with nearly 21 mil cells and a residual drop of $10^{-10}$ was deemed appropriate for the viscous analysis where the main interest was the solution on the surface of the model. A more in depth look into the quality of the mesh would have been done if the time and resources were available but the fact that a RANS solution was obtained for this research was considered a success. 

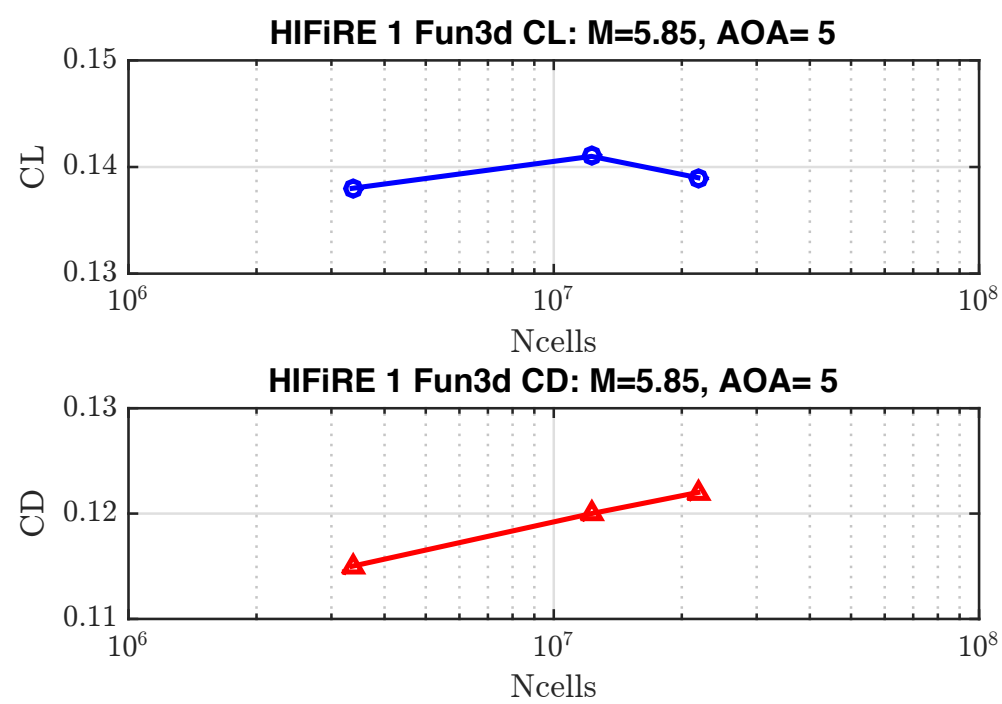

Figure 4.9: HIFiRE-1 FUN3D Grid Convergence Study for $C_{L}$ and $C_{D}$.

Results for the fine mesh are displayed below in Figures 4.10 4.14 . The surface $C_{p}$ contours can be seen in Figure 4.10, which show similar trends to that of Cart3D with UNLATCH, shown in Section 4.1, and CBAero with the stagnation locations being at the nose and the high-inclination region.

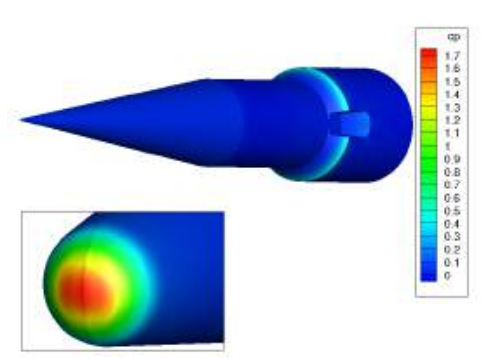

$M=5.85, \alpha=0$ degrees

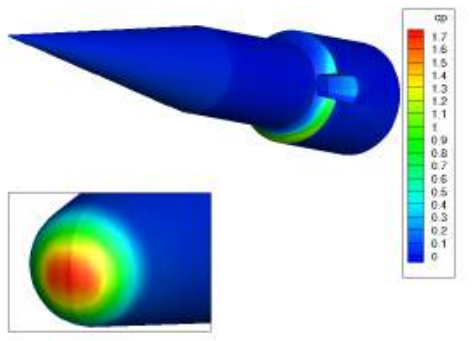

$M=5.85, \alpha=5$ degrees

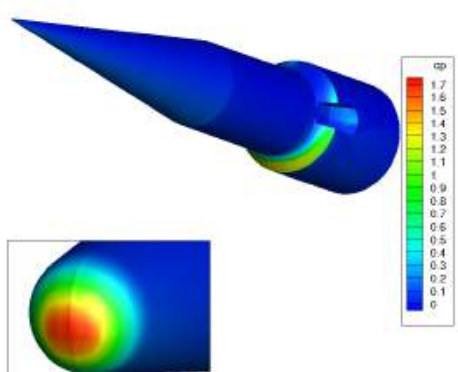

$M=5.85, \alpha=10$ degrees

Figure 4.10: HIFiRE-1 $C_{p}$ contours. 
The off-body Mach contours can be seen in Figure 4.11, showing the formation of the shock and expansion at positive angles of attack.

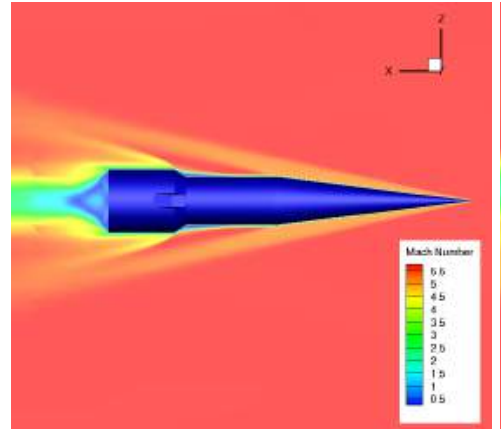

$M=5.85, \alpha=0$ degrees

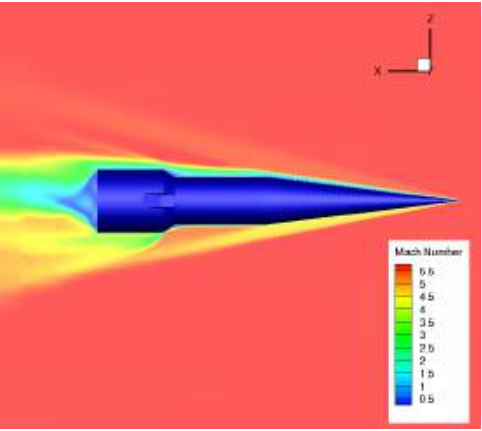

$M=5.85, \alpha=5$ degrees

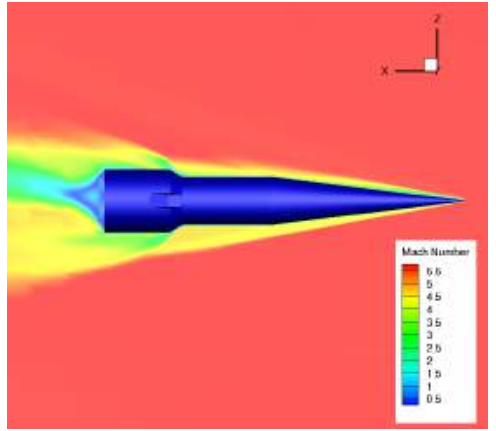

$M=5.85, \alpha=10$ degrees

Figure 4.11: HIFiRE-1 Mach contours.

Using the density contours displayed in Figure 4.12, one can infer similarities to the Cart3D results shown in Section 4.1

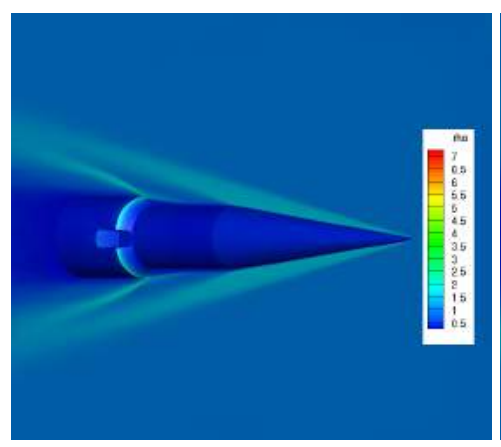

$M=5.85, \alpha=0$ degrees

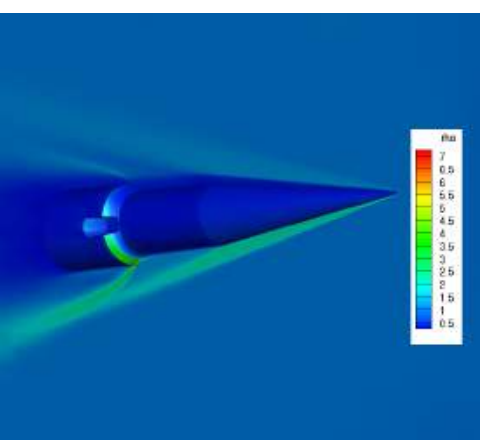

$M=5.85, \alpha=5$ degrees

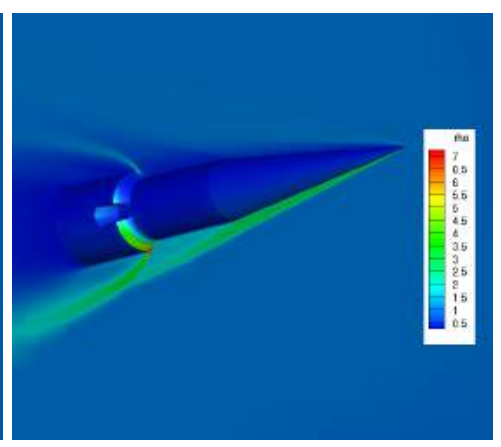

$M=5.85, \alpha=10$ degrees

Figure 4.12: HIFiRE-1 $\rho$ contours.

The y-plus values for the volume mesh in the boundary layer can be seen in Figure 4.13 to help judging the quality of the mesh. This can also be useful in determining areas in the solutions that 
degrade the overall flow solution. A y-plus value on the order of 1 was maintained throughout and was deemed sufficient for initial flow results.

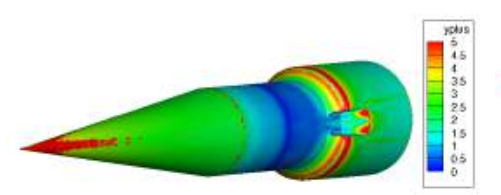

$$
M=5.85, \alpha=0 \text { degrees }
$$

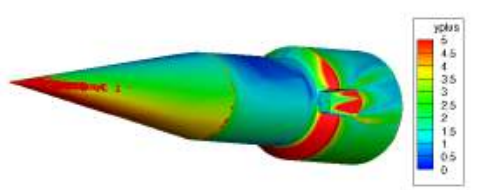

$M=5.85, \alpha=5$ degrees

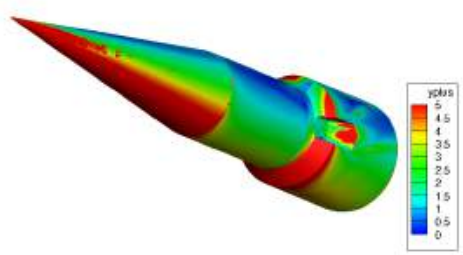

$M=5.85, \alpha=10$ degrees

Figure 4.13: HIFiRE-1 $y-$ plus contours.

The distribution of heating, identified through the heating rate shown in Figure 4.14, closely follows that of the results computed with UNLATCH (compare with Section4.1). A more concentrated heating at the stagnation point at the nose and overall higher magnitude for the heating was seen in the UNLATCH cases, which is expected when comparing an approximation (UNLATCH) to fully viscous solutions.

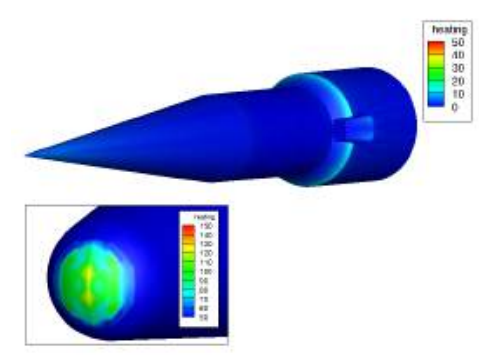

$M=5.85, \alpha=0$ degrees

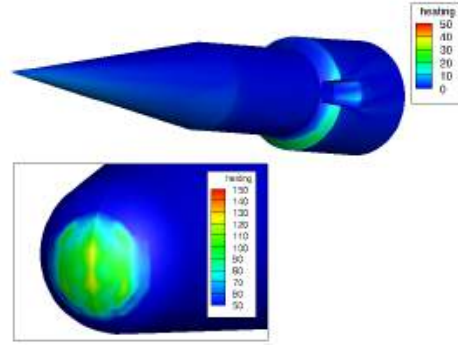

$M=5.85, \alpha=5$ degrees
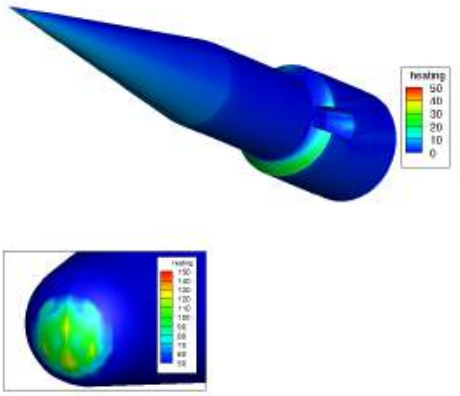

$M=5.85, \alpha=10$ degrees

Figure 4.14: HIFiRE-1 surface heating rates $\left[\frac{W}{m^{2}}\right]$. 


\section{CHAPTER V}

\section{EXPERIMENTAL AND NUMERICAL COMPARISON}

The comparison for the pressure coefficient values, $C_{p}$, from the wind tunnel experiments for the HIFiRE-1 model and the various computational results are shown in Figure 5.1. When the notch is at 90 degrees starboard, the instrumentation is directly in the flow direction, resulting in higher pressure readings. The flow solutions, resulting from the numerical models, were probed at the pressure tab location shown in Figure 2.7.

The trends for pressure tap 1 and 2 match well among the experimental and various computational values. At pressure tap 3, shock induced boundary layer separation causes a drop in surface pressure at the leeward 5 degree case, which, as expected, is not captured in the inviscid CART3D solution. The panel method, CBAero, with viscous approximations accurately predicts the drop in pressure at 5 degrees but does not predict the pressure recovery at 10 degrees when compared to the the experimental results. FUN3D seems to predict the experimental trends the best. The results for $P_{0}=2000$ psi and $T_{0}=1000 R(\mathrm{HT} / \mathrm{HP})$ as well as $P_{0}=700 \mathrm{psi}$ and $T_{0}=900 R(\mathrm{LT} / \mathrm{LP})$ are shown for comparisons since they represent the extreme ends of the test matrix.

The take away from this comparison was that for simple geometries, or at least simple portions of a geometries like $P_{T}=1,2$ for the HIFiRE-1 model, the viscous approximations with CBAero do an adequate job at predicting the surface pressure coefficient. This is with the understanding that when the surface is not at a significant angle with the flow, i.e $P_{T}=2$ CBAero over-predicts at these locations. Whenever re-attachment of the flow around the body occurs, at positive AOA 
for this model, a higher fidelity RANS solution may be beneficial to capture this complex flow phenomena.
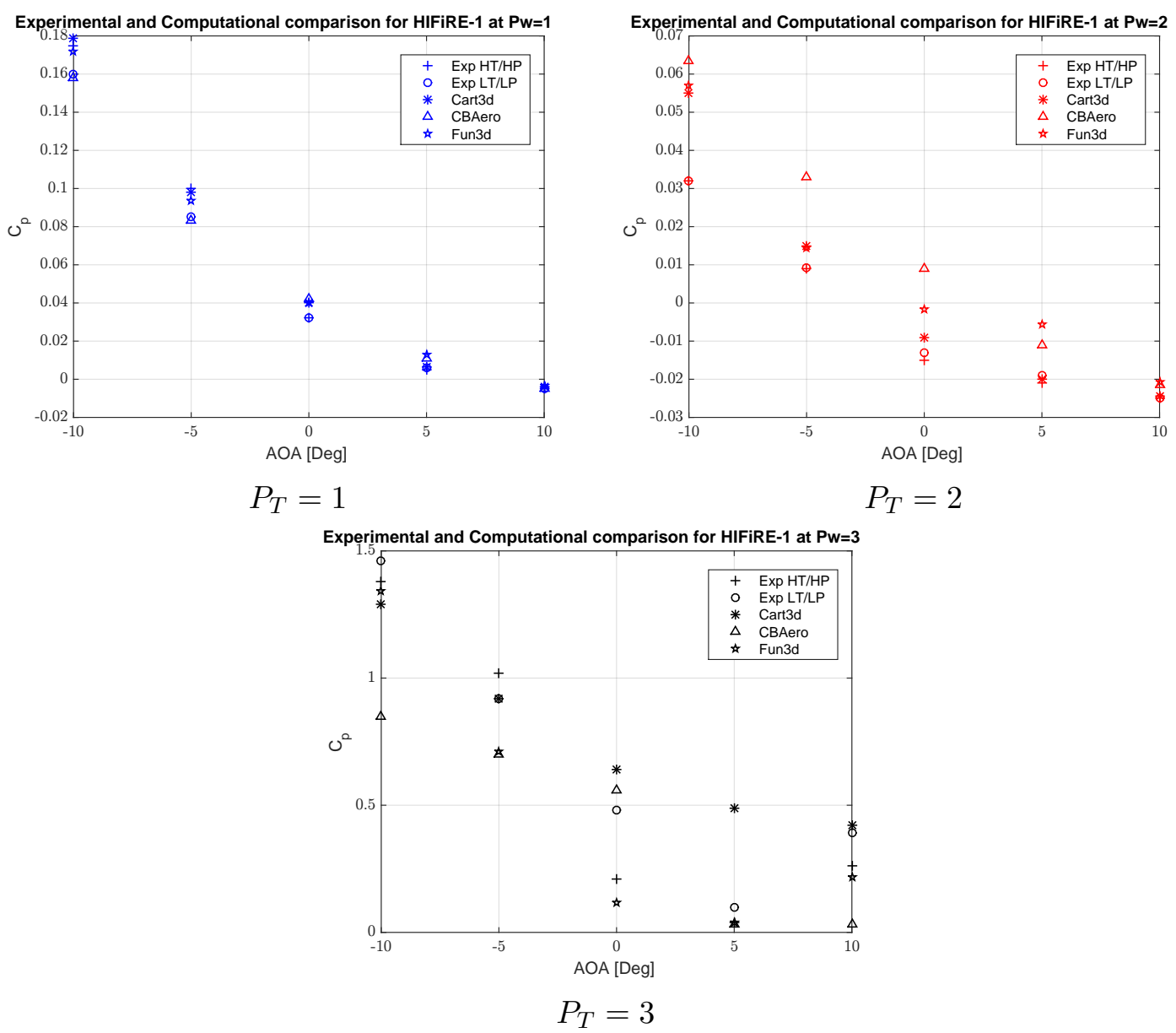

Figure 5.1: Comparison between experimental and computational $C_{p}$ results for HIFiRE-1 geometry.

The experimental vs. numerical results for the RFSG's eight pressure ports are shown in Figure 5.2. The trends between the experimental value for $C_{p}$ and the numerical results from Cart3D and CBAero seem to match at least in overall trend across the angle of attack sweep. With this model, the CBAero panel code with viscous approximations routinely over predicts the surface 
pressure coefficient but captures the over all trend across the various angles of attack. At the stagnation point, $P_{T}=1$, both numerical models overpredict $C_{p}$ but the relation between the two experimental runs shows good agreement. 

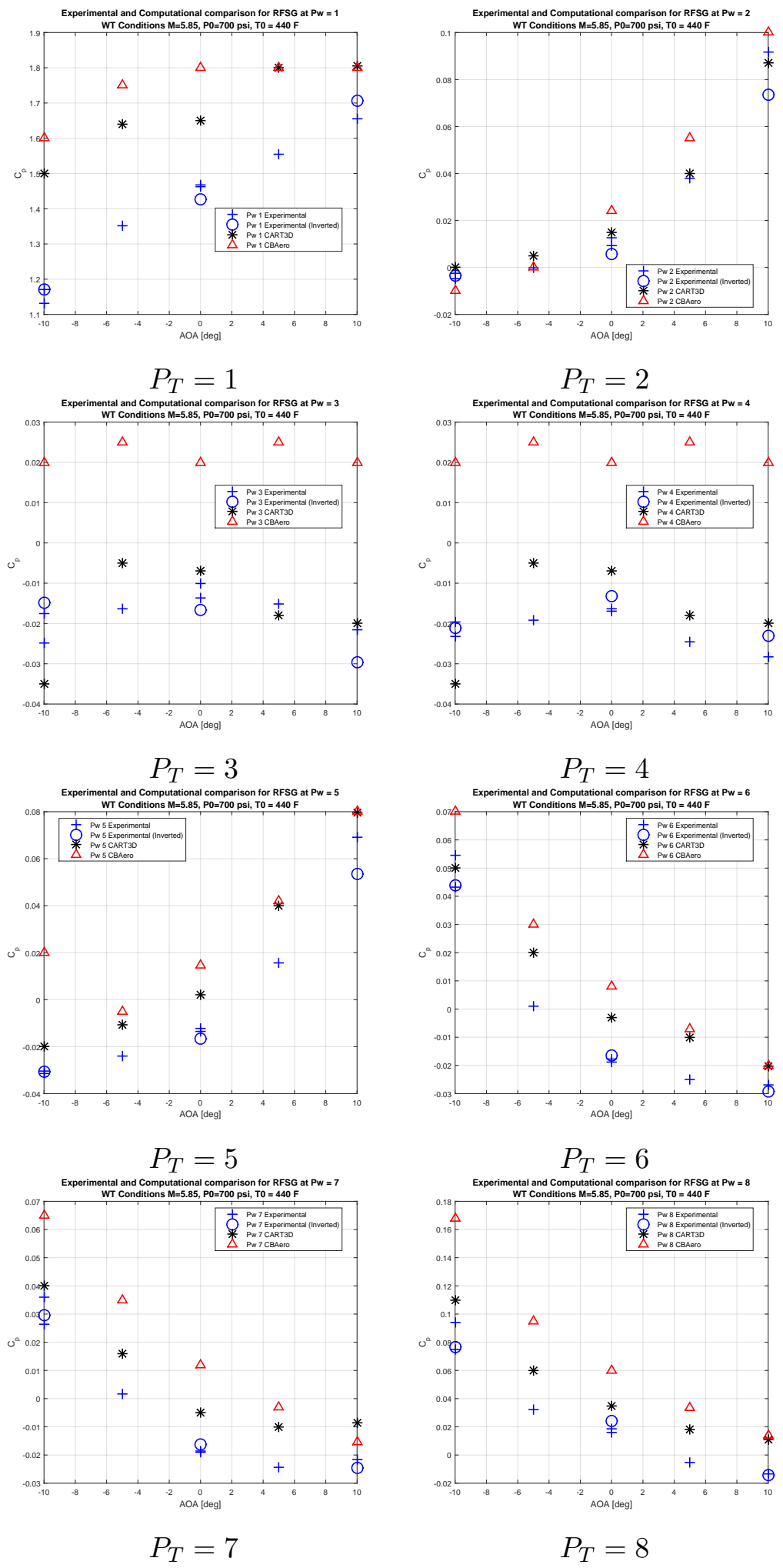

Figure 5.2: Comparison between experimental and computational $C_{p}$ results for RFSG geometry. 


\section{CHAPTER VI}

\section{CONCLUSION AND RECOMMENDATIONS}

Although one-to-one comparisons of experimental and computational surface temperature distributions at Mach 6 have not been accomplished, TSP data was obtained from the Mach 6 High Reynolds Number Facility at Wright-Patterson Air Force Base for the HIFiRE-1 payload geometry, the RFSG model and the GHV stingmount vehicle. The mere demonstration of TSP capability at Mach 6 is a significant step forward for experimental hypersonic vehicle design. Such a capability can lead to augmented design studies in the future such that insight into the thermal response of a geometry can be realized much earlier in the design process for hypersonic vehicles. Furthermore, though output temperature distributions could not yet be obtained from the UNLATCH boundary layer solver, the solver itself accepted flow solutions from Cart3D using a unique mesh adaptation strategy developed specifically for this effort. Once we can demonstrate valid wall temperature results from UNLATCH, there are powerful implications that can dramatically influence hypersonic vehicle design. The most notable implication is that the cost of reaching a wall temperature solution is effectively reduced to the cost of a single Euler flow solution. Breaching the barrier of the wall temperature problem of complex geometries in hypersonic flow using a boundary layer approximation combined with Euler solutions, through the use of UNLATCH and Cart3D, provides strong motivation to continue such work and to identify the problems slowing the progress in the application and implementation of UNLATCH. 


\subsection{Brief Overview of Project Accomplishments}

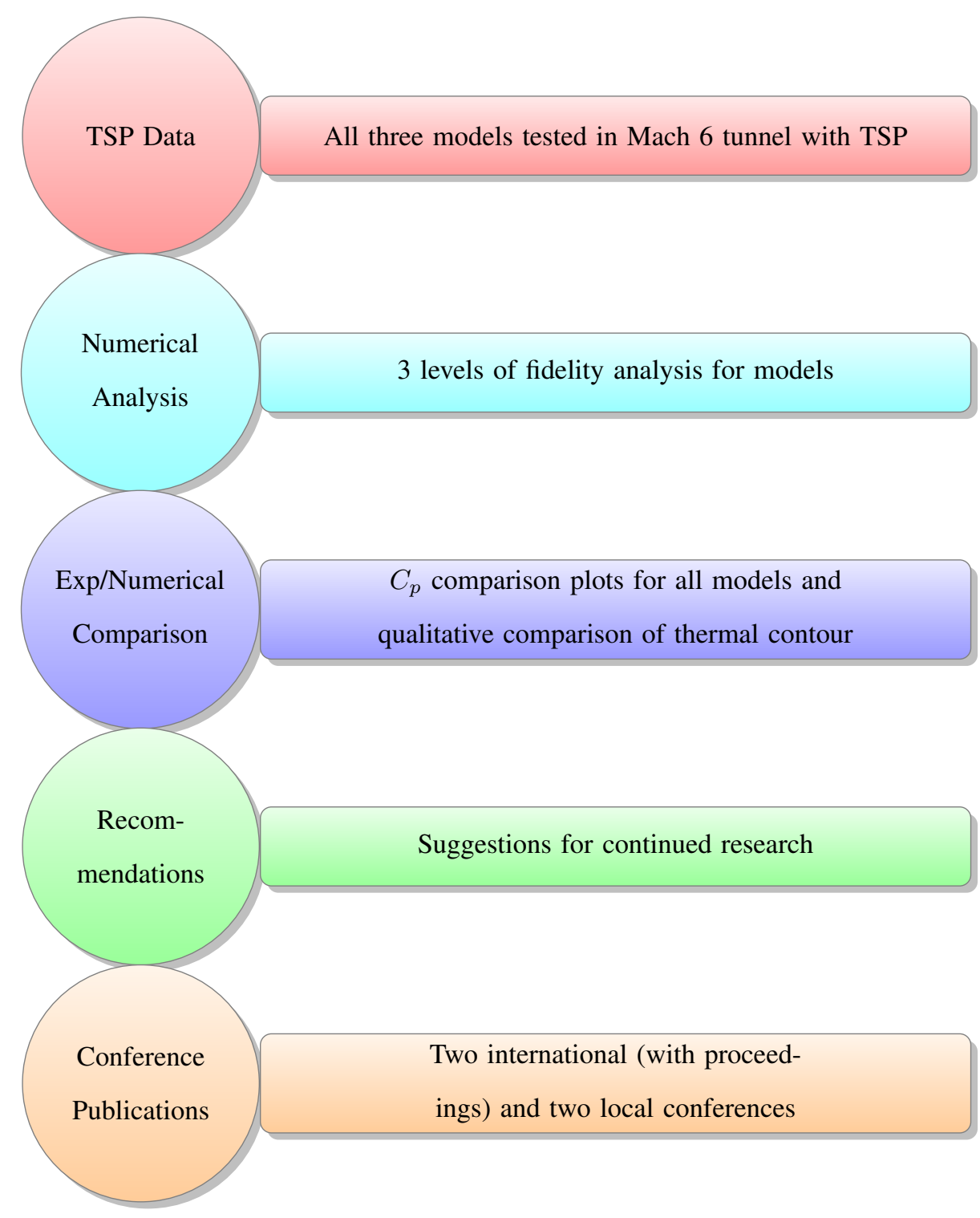

\subsection{Recommendations and Future Work}

The difficulty found in this research was the one-to-one comparison of the surface temperature contour, for a set wind tunnel condition, between the numerical and experimental analysis with TSP. Since the wind tunnel testing could not be done to steady state, quantifying the magnitude for the temperature on the model from computational analysis proved difficult in that the conduction 
into the model would have to be incorporated into the simulation. This would lead to the need for modeling the fluid-structure interaction which was outside the scope of this project. In terms of recommendations, in order to test the capability of the Mach 6 tunnel for obtaining aero-thermal data, the models should have been constructed as thermal models where the thinness of the wings did not result in almost immediate conduction. This condition makes it difficult to determine where the heat, shown in the rise of luminosity of the paint, came from. Also with the way the models were built, the heat transfer coefficient could not be obtained and would have been a better comparison for the numerical analysis. Although there were some shortcomings, the fact that TSP contours were obtained for all three experimental models and numerical results of varying fidelity were computed to quantify the abilities of the tunnel are hopefully useful to the hypersonic community.

The main objectives of the project were reached in that an estimation of the qualitative view of the aero-thermal distribution for complex aircraft could be determined using TSP in the Mach 6 wind tunnel at Wright-Patterson AFB. In terms of directly comparing the magnitude of the temperature readings to numerical simulations, the way in which the experimental models and "thermal" models were constructed did not allow for accurate thermal data, like heat transfer rate, to be obtained for the purpose of comparing to numerical analysis. 


\section{Research Publications}

"Hypersonic Experimental Aero-thermal Capability Study through Multilevel Fidelity Computational Fluid Dynamics” Denton G. Sagerman, James Tancred, Markus Rumpfkeil, and Barry M. Hellman, 34th AIAA Applied Aerodynamics Conference (2016) doi: 10.2514/6.2016-3577

"Comparisons of Measured and Modeled Aero-thermal Distributions for Complex Hypersonic Configurations” Denton G. Sagerman, Markus P. Rumpfkeil, Barry M. Hellman, and Nastassja Dasque, 55th AIAA Aerospace Sciences Meeting (2017) doi: 10.2514/6.2017-0264

\section{Conference Presentations}

41st Dayton-Cincinnati Aerospace Sciences Symposium (March 2, 2016) ”Hypersonic Experimental Aero-thermal Capability Study” by Denton G. Sagerman (Presenter), Markus P. Rumpfkeil, Barry Hellman

AIAA Aviation 2016: Applied Aerodynamics Conference (June 14, 2016) "Hypersonic Experimental Aero-thermal Capability Study through Multilevel Fidelity Computational Fluid Dynamics” by Denton G. Sagerman (Presenter), Markus P. Rumpfkeil, Barry Hellman

AIAA SciTech 2017: Hypersonic Aerodynamics (January 9, 2017): "Comparisons of Measured and Modeled Aero-thermal Distributions for Complex Hypersonic Configurations" by Denton G. Sagerman, Markus P. Rumpfkeil (Presenter), Barry Hellman

42nd Dayton-Cincinnati Aerospace Sciences Symposium (March 1, 2017) ”Comparisons of Measured and Modeled Aero-thermal Distributions for Complex Hypersonic Configurations" by Denton G. Sagerman (Presenter), Markus P. Rumpfkeil, Barry Hellman 


\section{FIELDS OF STUDY}

Hypersonic Computational Fluid Dynamics

Studies in:

$\begin{array}{ll}\text { Computational Fluid Dynamics } & \text { Dr. Markus Rumpfkeil } \\ \text { Experimental Fluid Mechanics } & \text { Dr. Aaron Altman } \\ \text { Aeroelasticity } & \text { Dr. Ray Kolonay } \\ \text { Orbital Mechanics } & \text { Dr. Kerry Hicks } \\ \text { Hypersonic Aerodynamics } & \text { Dr. Jose Camberos } \\ \text { Propulsion } & \text { Dr. Mark Durbin } \\ \text { Special Topics in CFD } & \text { Dr. Markus Rumpfkeil } \\ \text { Probability and Statistics for Engineers } & \text { Dr. Kelly Schneider }\end{array}$




\section{BIBLIOGRAPHY}

[1] D. R. P. Hallion, "The History of Hypersonics: or, "Back to the Future - Again and Again"," AIAA Paper, 2005-329, 2005.

[2] NASA, "NASA Armstrong Fact Sheet: X-15 Hypersonic Research Program," http://www.nasa.gov/centers/armstrong/news/FactSheets/FS-052-DFRC.html, 28 Feb 2014.

[3] K. Berger and S. Rufer, "NASA Langley Aerothermodynamics Labratory: Hypersonic Testing Capabilities," AIAA SciTech, vol. Accepted. 6.2015-1337, 2015.

[4] J. Hayes and S. Altman, "Pressure-Sensitive Paint (PSP) and Temperature-Sensitive Paint (TSP) Testing on Pitot probes in Supersonic Flow," AFRL-Rq-WP-TR-2014-0262, 2014.

[5] — - "AFRL/RQ Mach 3 and Mach 6 High Re Facility," AIAA Dayton-Cincinnati Section Lunch and Learn, 2014.

[6] “Tecplot 360 EX 2015 R1,” Tecplot, Inc., 2015.

[7] J. D. Anderson, Hypersonic and High-Temperature Gas Dynamics. AIAA, 2006.

[8] R. Launius, "Hypersonic Flight: Evolution from X-15 to Space Shuttle," AIAA/ICAS International Air and Space Symposium and Exposition, 2003.

[9] J. A. Walker and J. Weil, “The X-15 Program,” NASA Flight Research Center Edwards, California.

[10] J. D. Anderson, Computational Fluid Dynamics - The Basics with Applications. McGrawHill, 1995.

[11] J. S. M. Joseph M. Hank and R. C. Mutzman, "The X-51A Scramjet Engine Flight Demonstration Program," 15th AIAA International Space Planes and Hypersonic Systems and Technologies Conference, 2008.

[12] R. Hoffman, "MACH 6 HIGH REYNOLDS FACILITY WIND TUNNEL MODELS STRESS ANALYSIS REPORT,” University of Dayton Research Institute Technical Report, 2017.

[13] R. L. Kimmel and D. Adamczak, "HIFiRE-1 Background and Lessons Learned," AIAA, vol. Accepted. 2012-1088, 2012. 
[14] J. S. 1Lt Brent Ruttle and G. Liston, "Generic Hyersonic Vehicles for Conceptual Design Analyses,” AFRL/RQHT, 2130 Eighth Street, WPAFB, OH 45433-7542 USA, 2012.

[15] P. David J. Kinney, “CBAERO 4.1+ User's Manual Version 1.2,” NASA Ames Research Center: CMB-2008-9, September 2012.

[16] M. Nemec and M. J. Aftosmis, "Adjoint-Based Adaptive Mesh Refinement for Complex Geometries," AIAA Paper, 2008-0725, 2008.

[17] M. J. Aftosmis, M. J. Berger, and J. E. Melton, "Robust and Efficient Cartesian Mesh Generation for Component-Based Geometry," AIAA Paper, 97-0196, 1997.

[18] I. Desktop Aeronautics, "Quick Start Guide to Cart3D," DA v1.2 based on NASA v1.3.6, 2 May 2012.

[19] “Pointwise GridGen Ver. 15.17,” Pointwise, Inc., 2011.

[20] “Numeca HEXPRESS/Hybrid v3.1,” Numeca International, 2013.

[21] M. Nemec and M. J. Aftosmis, "Adjoint Error Estimation and Adaptive Refinement for Embedded-Boundary Cartesian Meshes," AIAA Paper, 2007-4187, 2007.

[22] K. J. W. H. H. Hamilton and F. R. DeJarnette, "Improved Approximate Method for Computing Convective Heating on Hypersonic Vehicles Using Unstructured Grids," AIAA, vol. Accepted. 2006-3394, 2006.

[23] R. B. J. Carlson, J. Derlaga, P. Gnoffo, D. Hammond, W. Jones, B. Kleb, E. Lee-Rausch, E. Nielsen, M. Park, C. Rumsey, J. Thomas, and W. Wood, "FUN3D Manual: 12.7," NASA Technical Memorandum 218761, 2015.

[24] “Numeca HEXPRESS/View v3.1,” Numeca International, 2013.

[25] N. International, "User Manual: HEXPRESS v3.1 Unstructured Grid Generator," Numerical Mechanics Applications, September 2013.

[26] “IcemCFD v17.2 Revision 24836,” ANSYS, 2015. 


\section{APPENDIX A}

HIFiRE-1 Experimental Results 

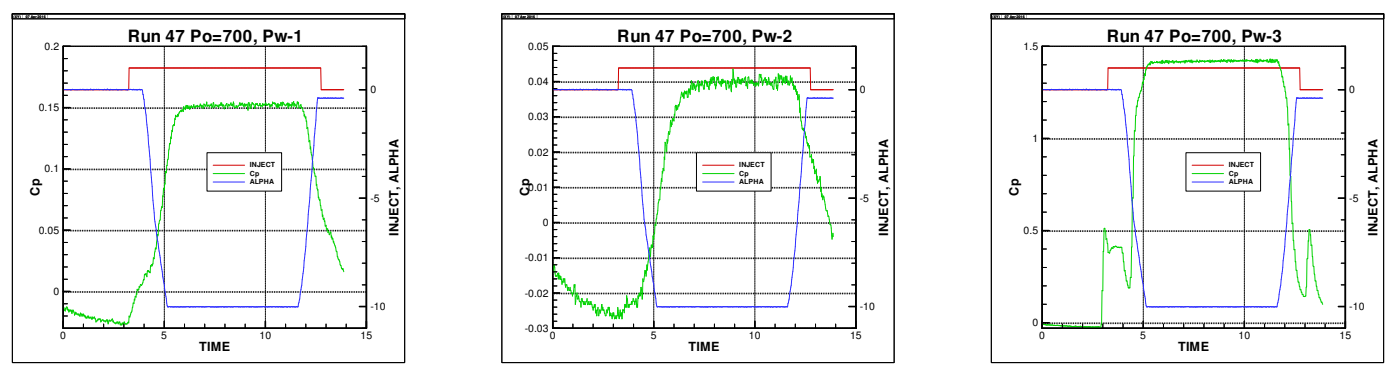

$P_{T}=1, A O A=-10$
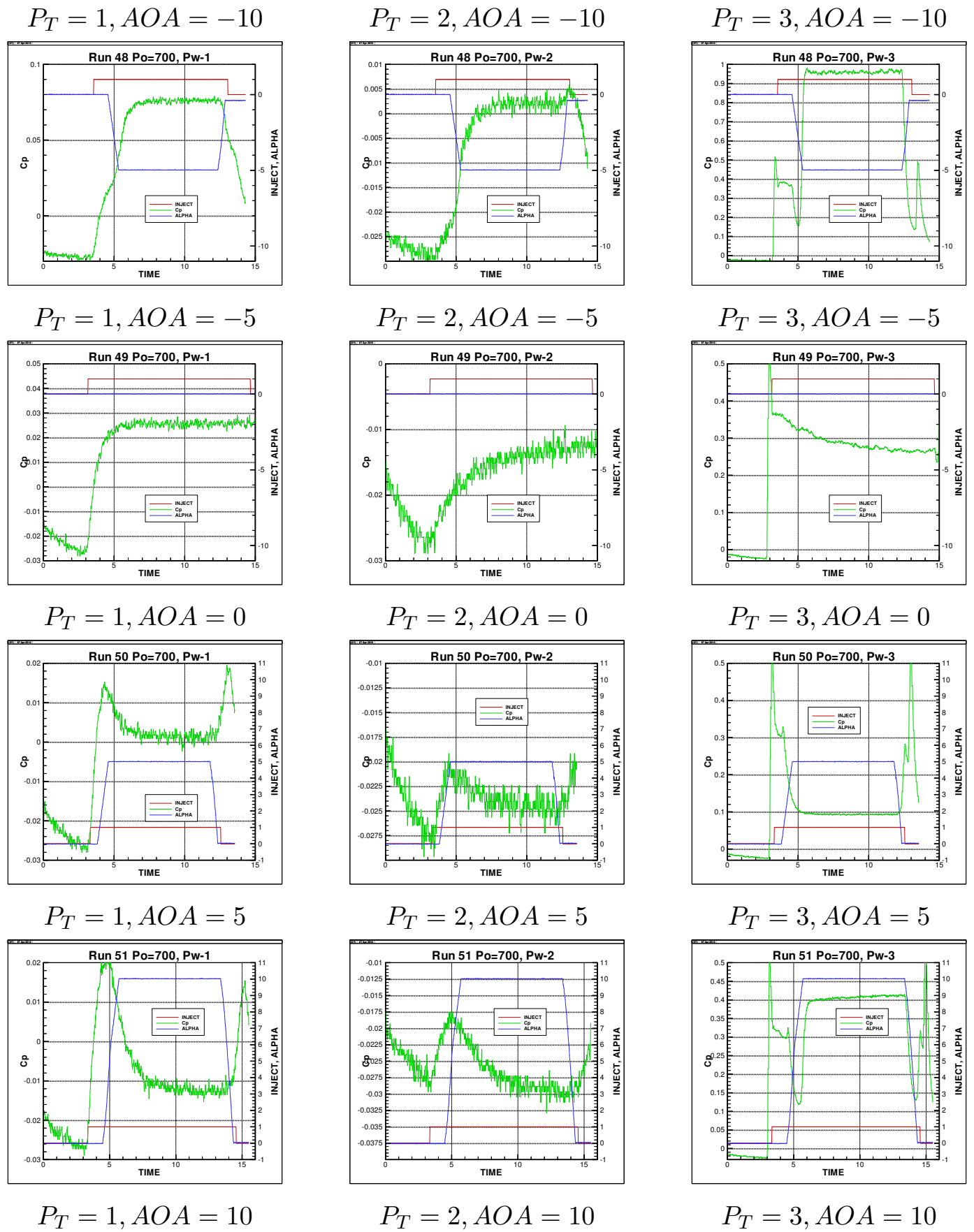

Figure A.1: Mach 6 Wind Tunnel $C_{p}$ results for $P_{0}=700 \mathrm{psi} T_{0}=1000 \mathrm{R}$ at $A O A=-10,-5,0,5,10$ deg with Notch starboard. 

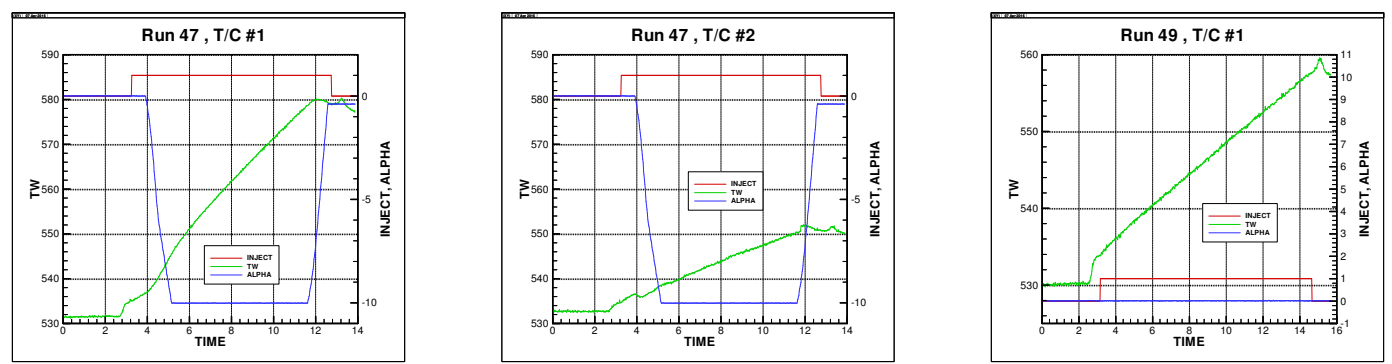

$T_{C}=1, A O A=-10$

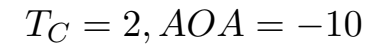

$$
T_{C}=1, A O A=0
$$
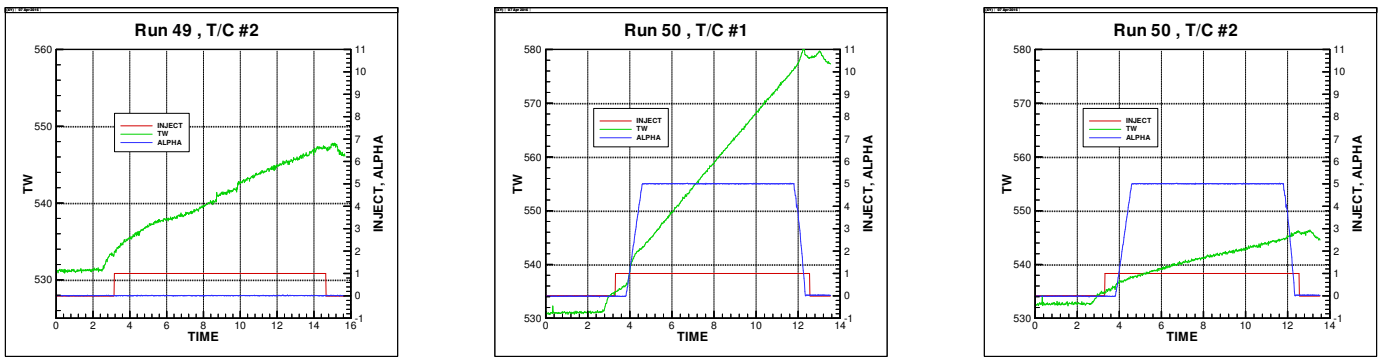

$$
T_{C}=2, A O A=0
$$

$$
T_{C}=1, A O A=5
$$

$T_{C}=2, A O A=5$

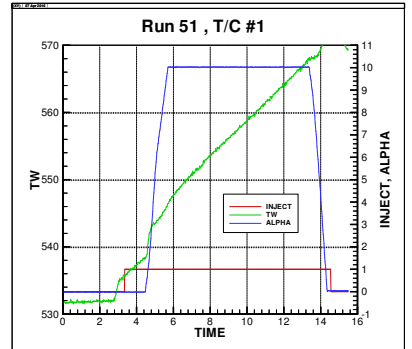

$T_{C}=1, A O A=10$

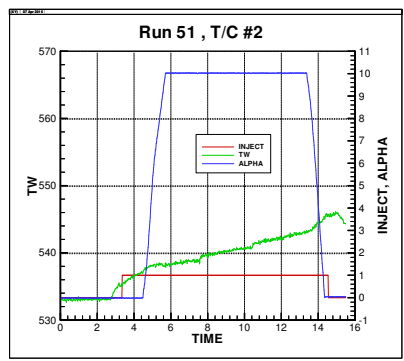

$T_{C}=2, A O A=10$

Figure A.2: Mach 6 Wind Tunnel temperature results for $P_{0}=700$ psi $T_{0}=1000 \mathrm{R}$ at $A O A=$ $-10,-5,0,5,10$ deg with Notch starboard. 

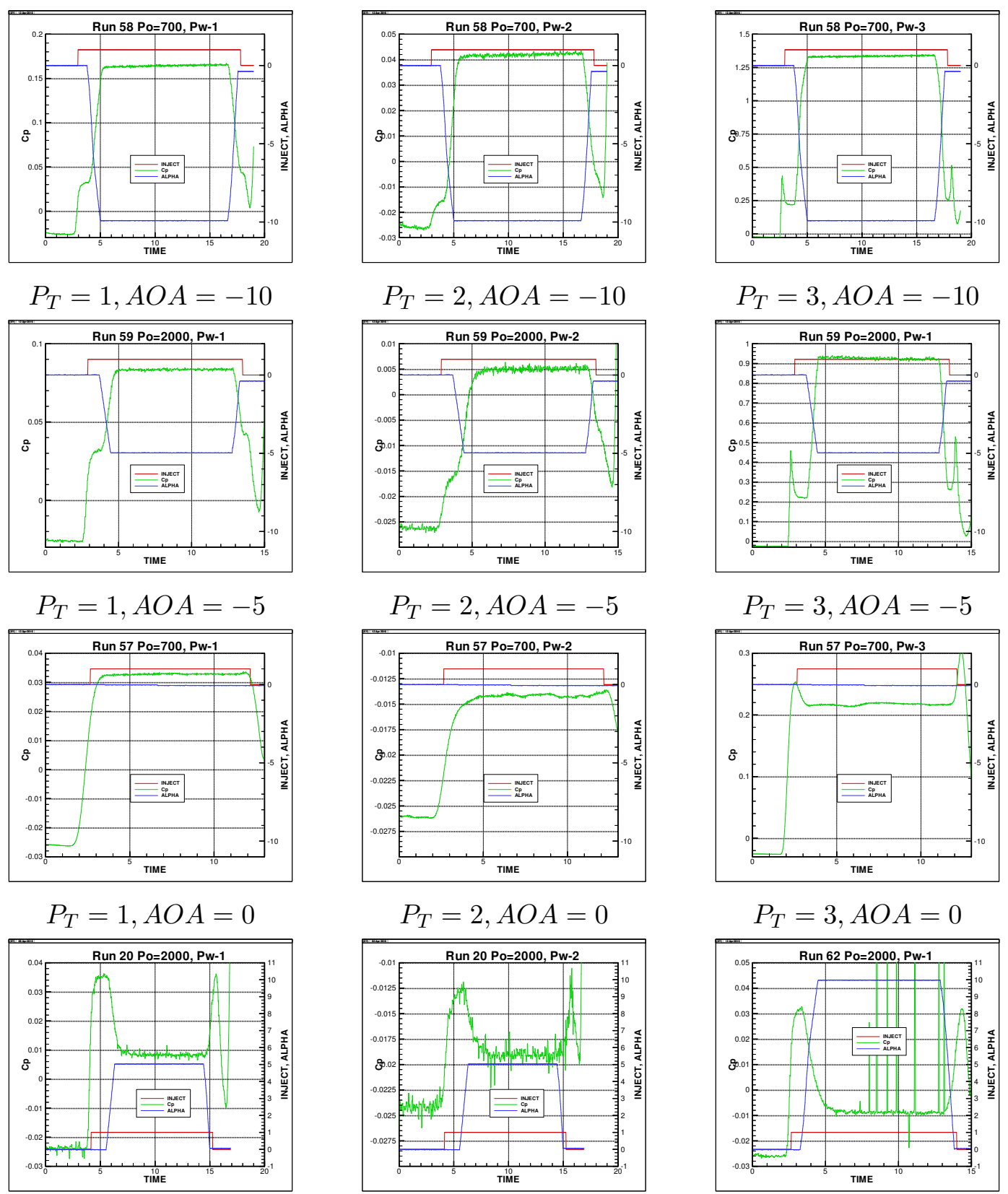

$$
P_{T}=1, A O A=5
$$

$$
P_{T}=2, A O A=5
$$

$$
P_{T}=1, A O A=10
$$

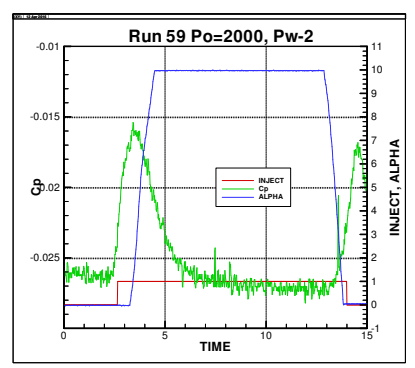

$P_{T}=2, A O A=10$

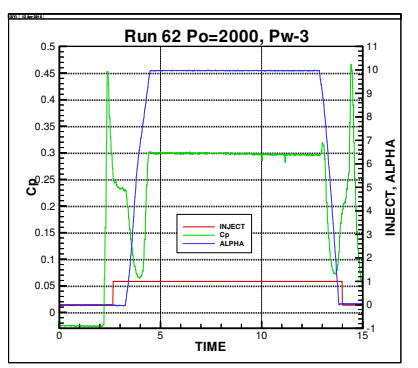

$P_{T}=3, A O A=10$

Figure A.3: Mach 6 Wind Tunnel $C_{p}$ results for $P_{0}=2000 \mathrm{psi} T_{0}=900 \mathrm{R}$ at $A O A=-10,-5,0,5,10$ deg with Notch starboard. 

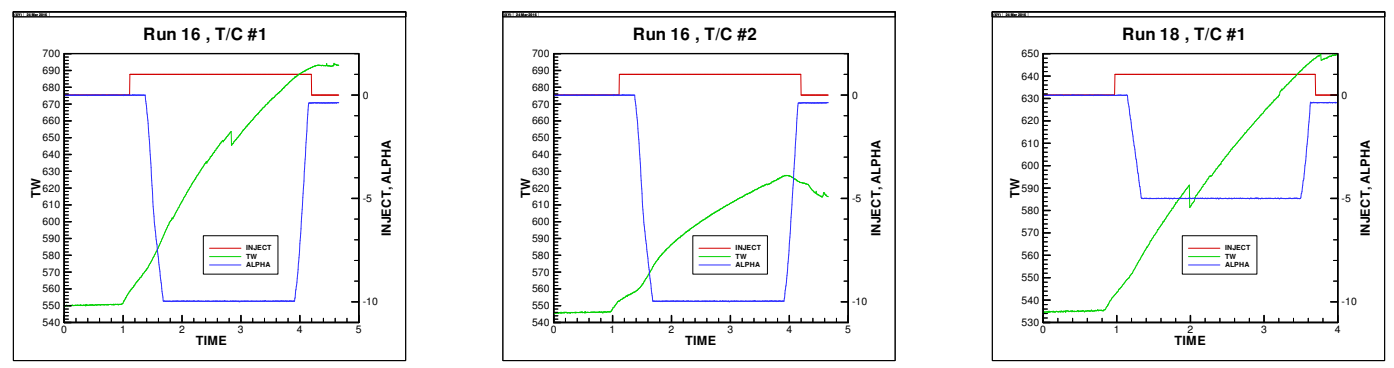

$T_{C}=1, A O A=-10$

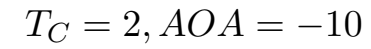

$T_{C}=1, A O A=-5$
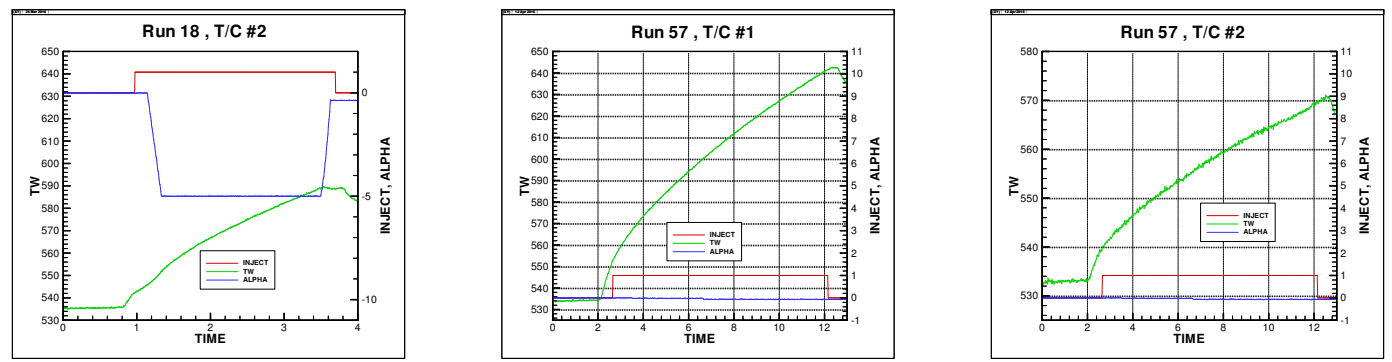

$$
T_{C}=2, A O A=-5
$$

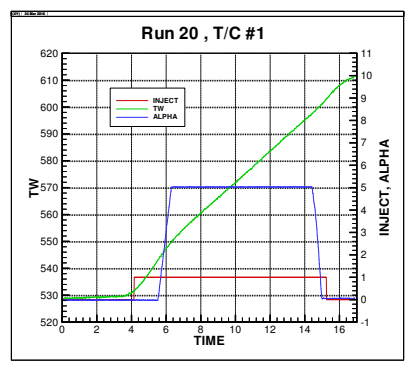

$$
T_{C}=1, A O A=0
$$

$$
T_{C}=2, A O A=0
$$

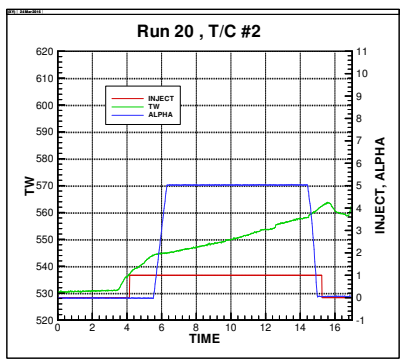

$$
T_{C}=1, A O A=5
$$

\section{$T_{C}=2, A O A=5$}
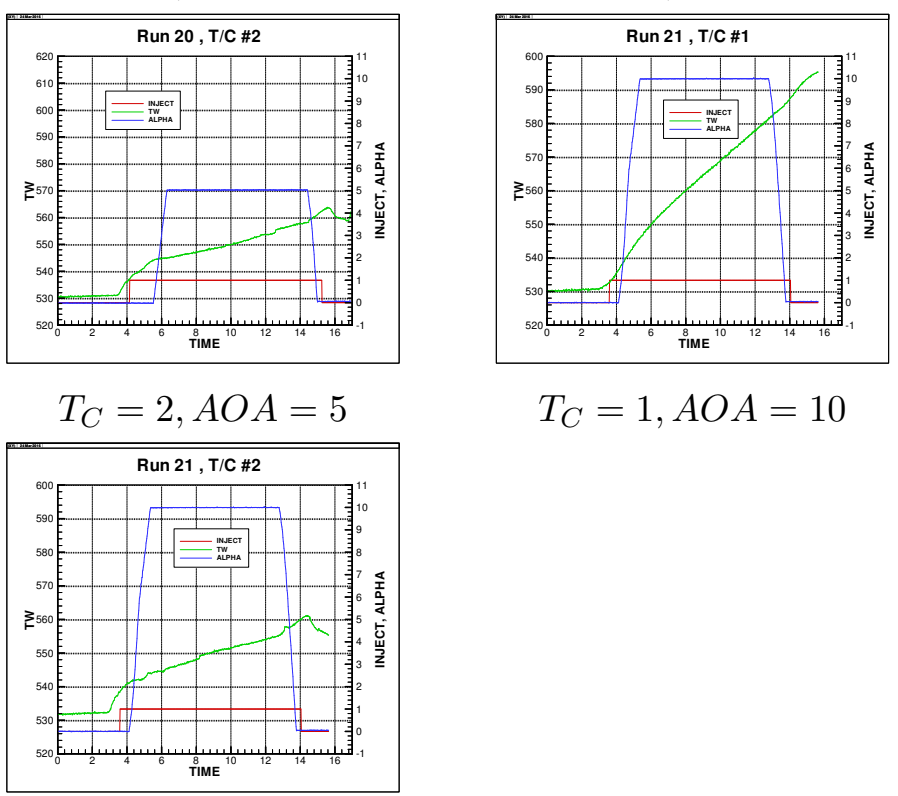

$$
T_{C}=1, A O A=10
$$

$$
T_{C}=2, A O A=10
$$

Figure A.4: Mach 6 Wind Tunnel temperature results for $P_{0}=2000$ psi $T_{0}=900 \mathrm{R}$ at $A O A=$ $-10,-5,0,5,10$ deg with Notch starboard. 

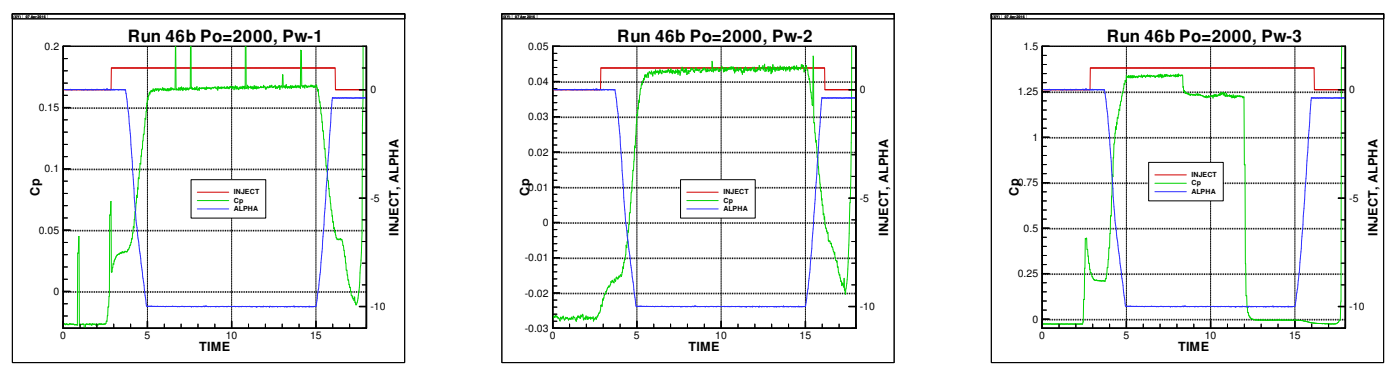

$P_{T}=1, A O A=-10$
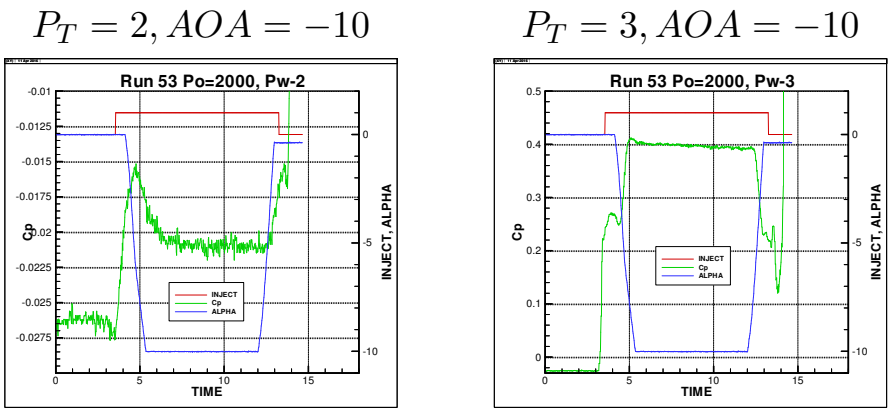

$$
P_{T}=1, A O A=-5
$$

$$
P_{T}=2, A O A=-5
$$

$$
P_{T}=3, A O A=-5
$$
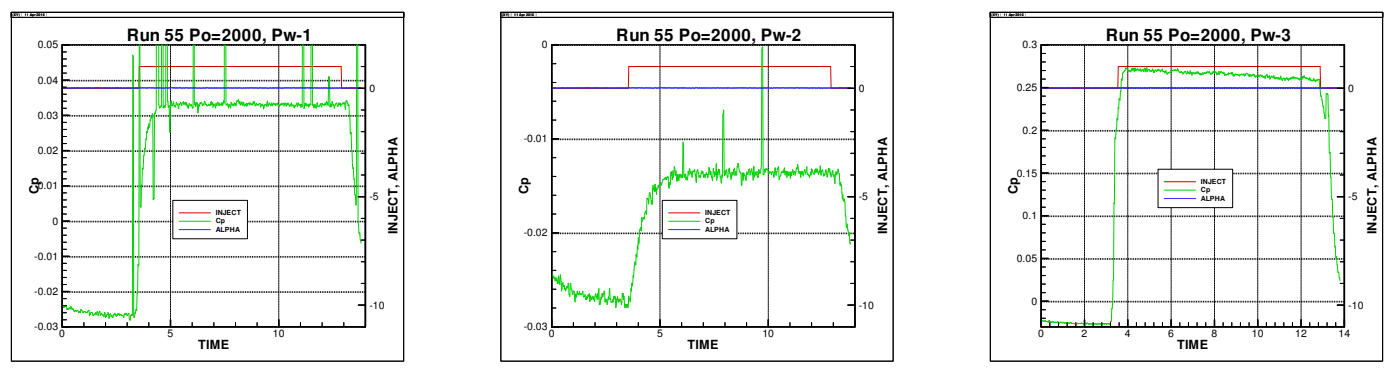

$$
P_{T}=1, A O A=0
$$

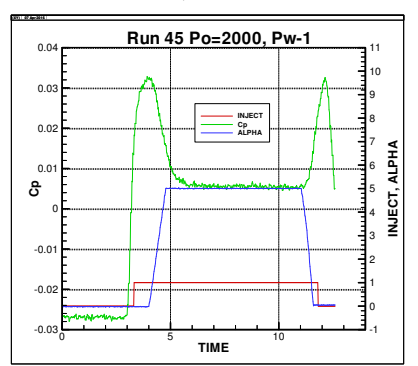

$$
P_{T}=2, A O A=0
$$
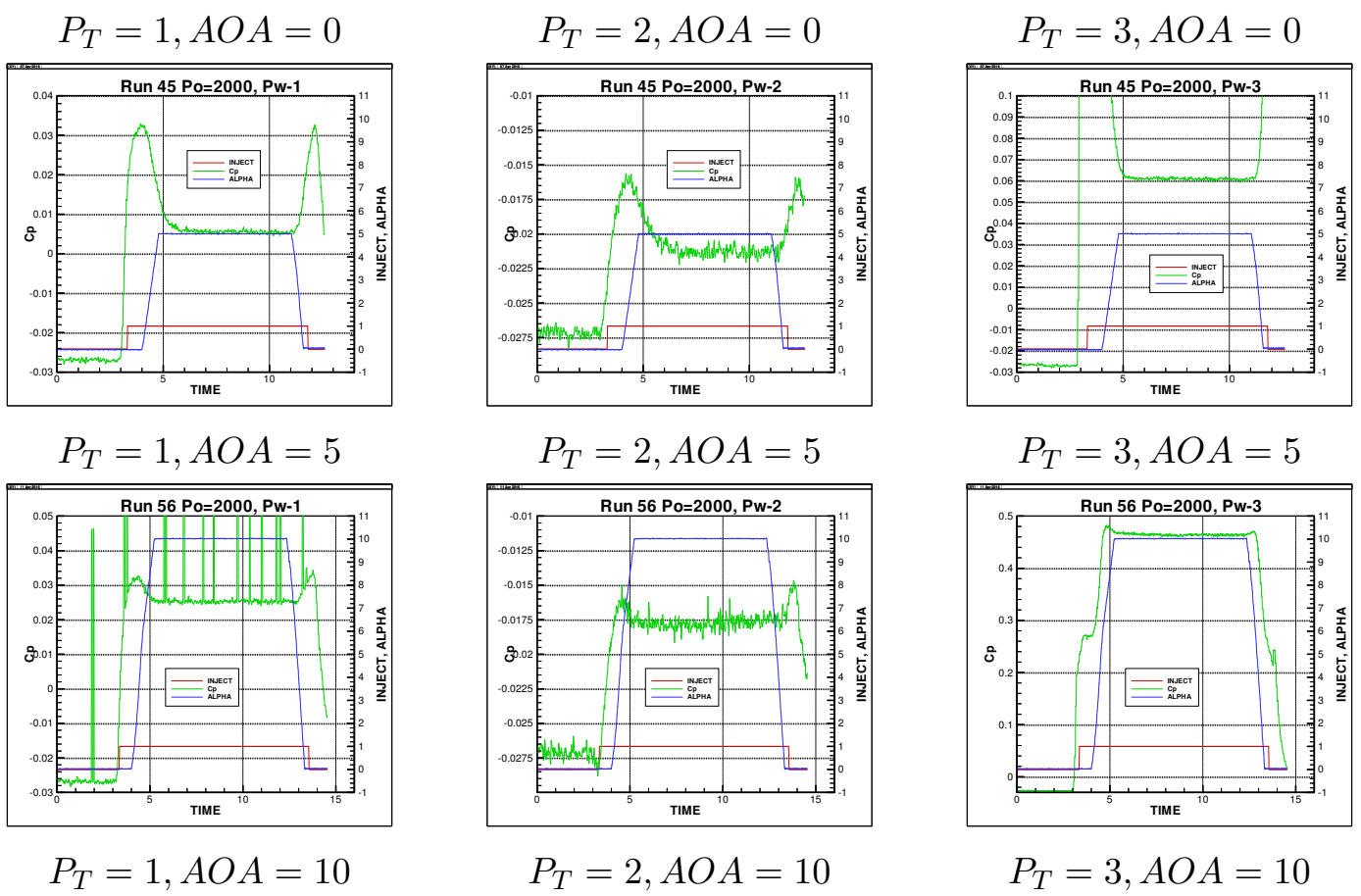

Figure A.5: Mach 6 Wind Tunnel $C_{p}$ results for $P_{0}=2000$ psi $T_{0}=1000 \mathrm{R}$ at $A O A=-10$,$5,0,5,10$ deg with Notch starboard. 

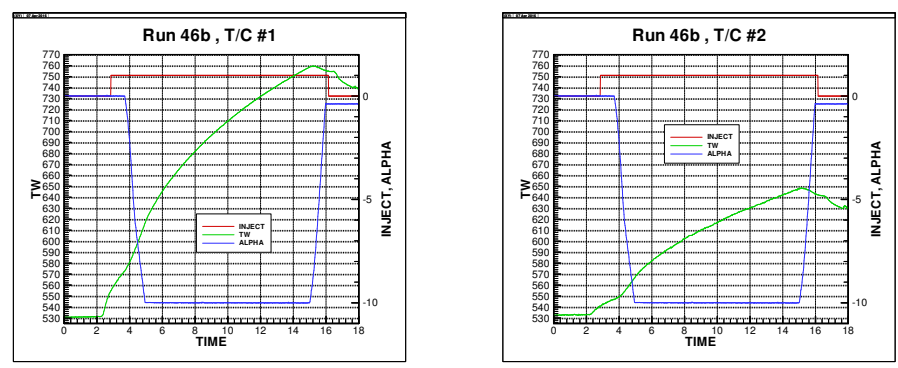

$T_{C}=1, A O A=-10$
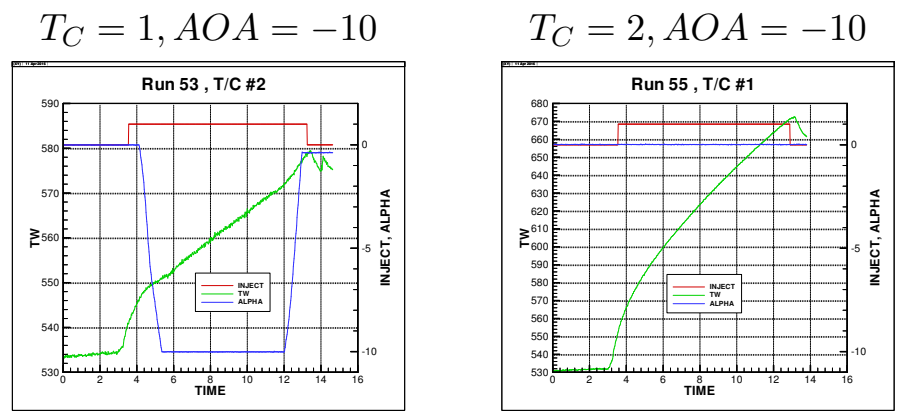

$T_{C}=2, A O A=-5$

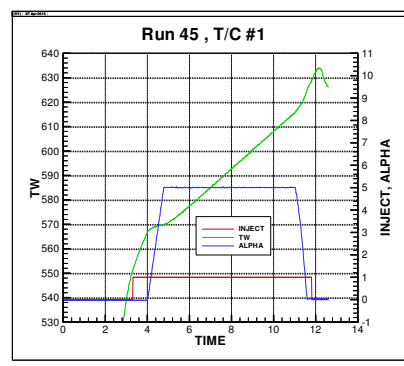

$T_{C}=1, A O A=5$

$$
T_{C}=1, A O A=0
$$

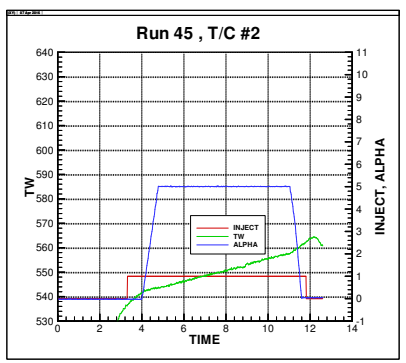

$$
T_{C}=2, A O A=5
$$

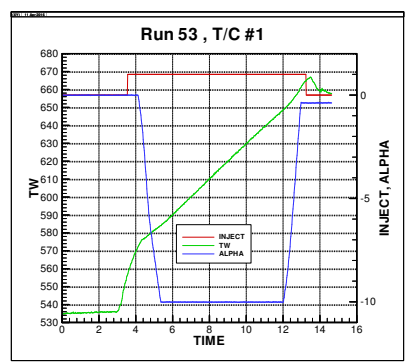

$T_{C}=1, A O A=-5$
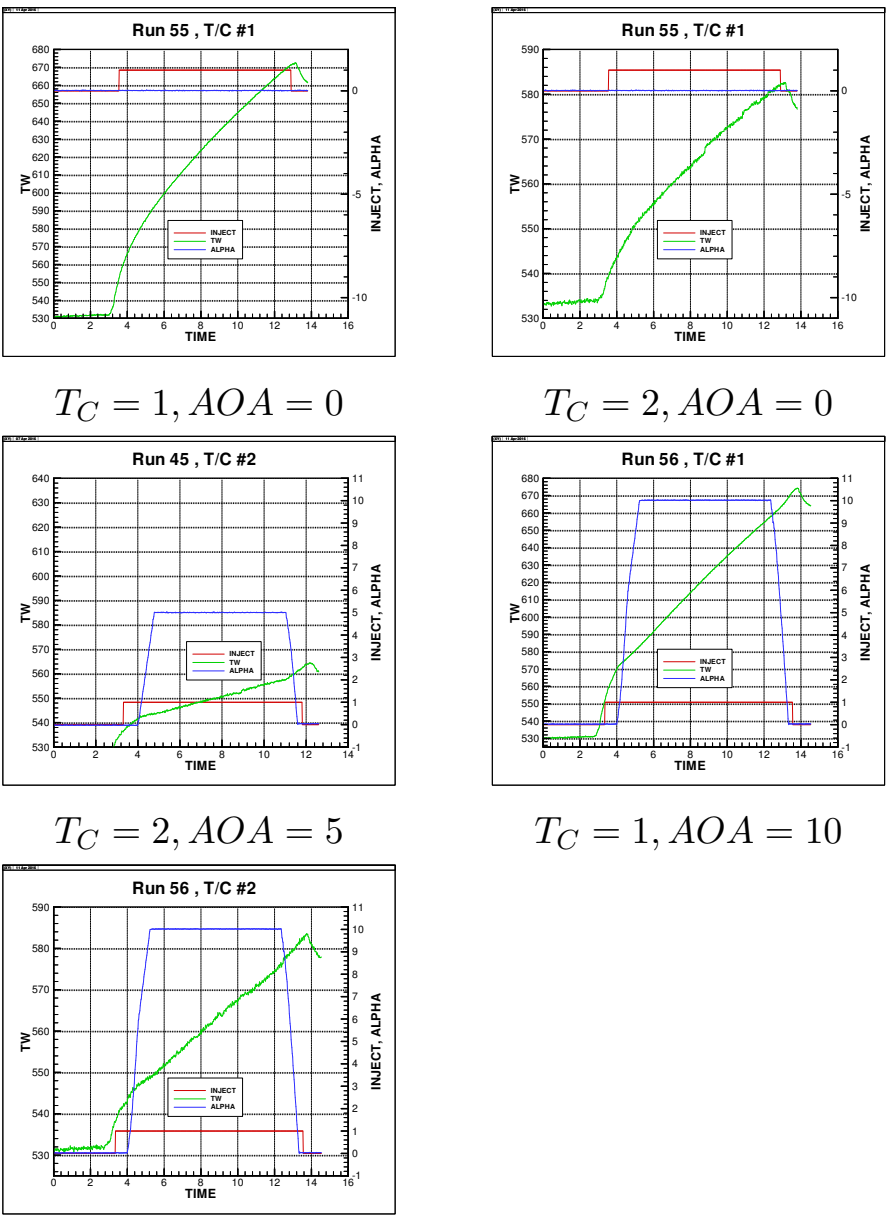

$$
T_{C}=2, A O A=10
$$

$$
T_{C}=2, A O A=0
$$

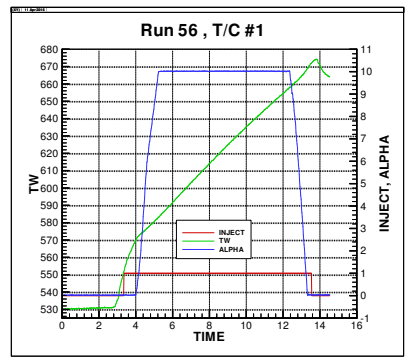

$T_{C}=1, A O A=10$

Figure A.6: Mach 6 Wind Tunnel temperature results for $P_{0}=2000 \mathrm{psi} T_{0}=1000 \mathrm{R}$ at $A O A=$ $-10,-5,0,5,10$ deg with Notch starboard. 


\section{APPENDIX B}

\section{Numeca Hexpress/Hybrid Input Script}

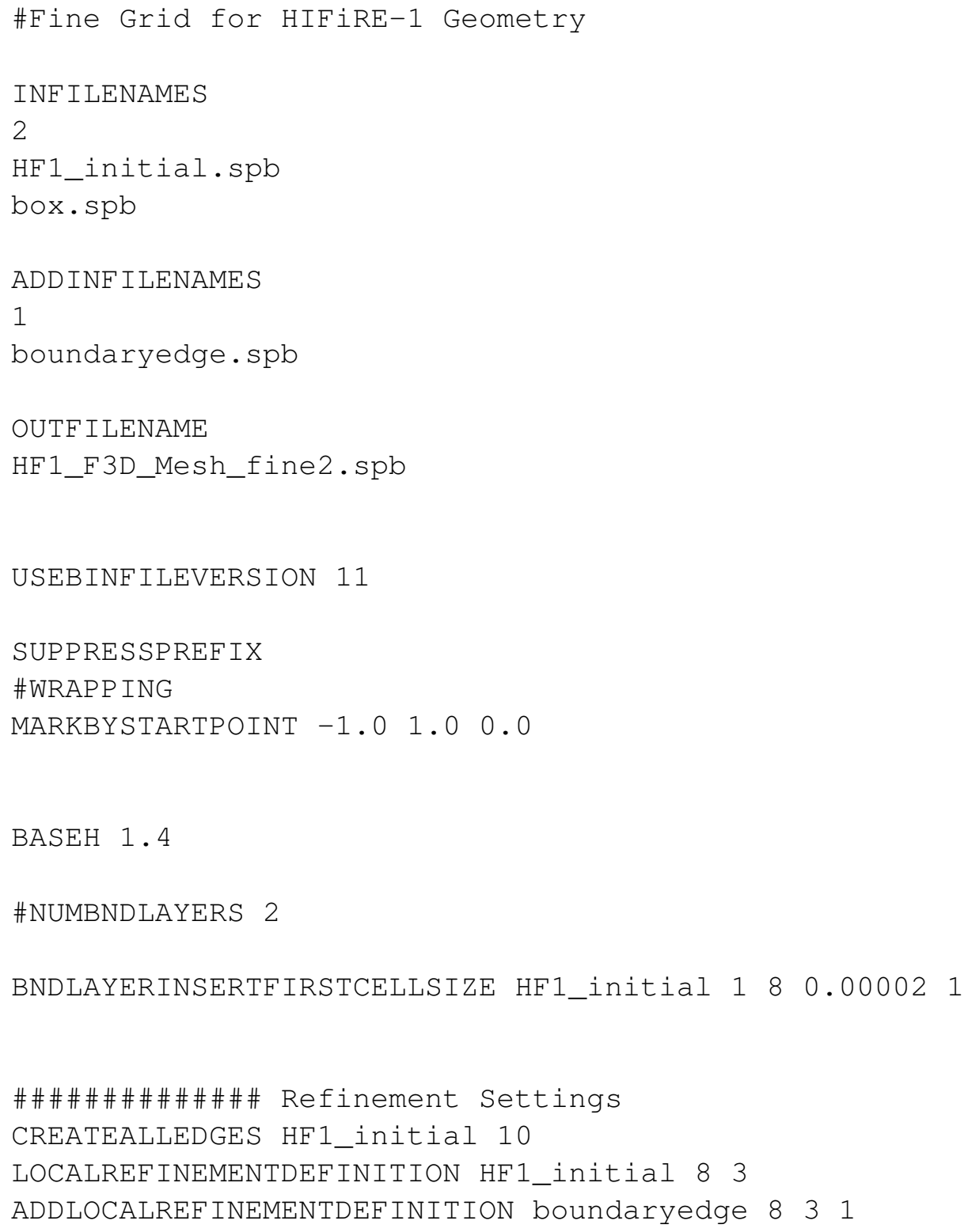




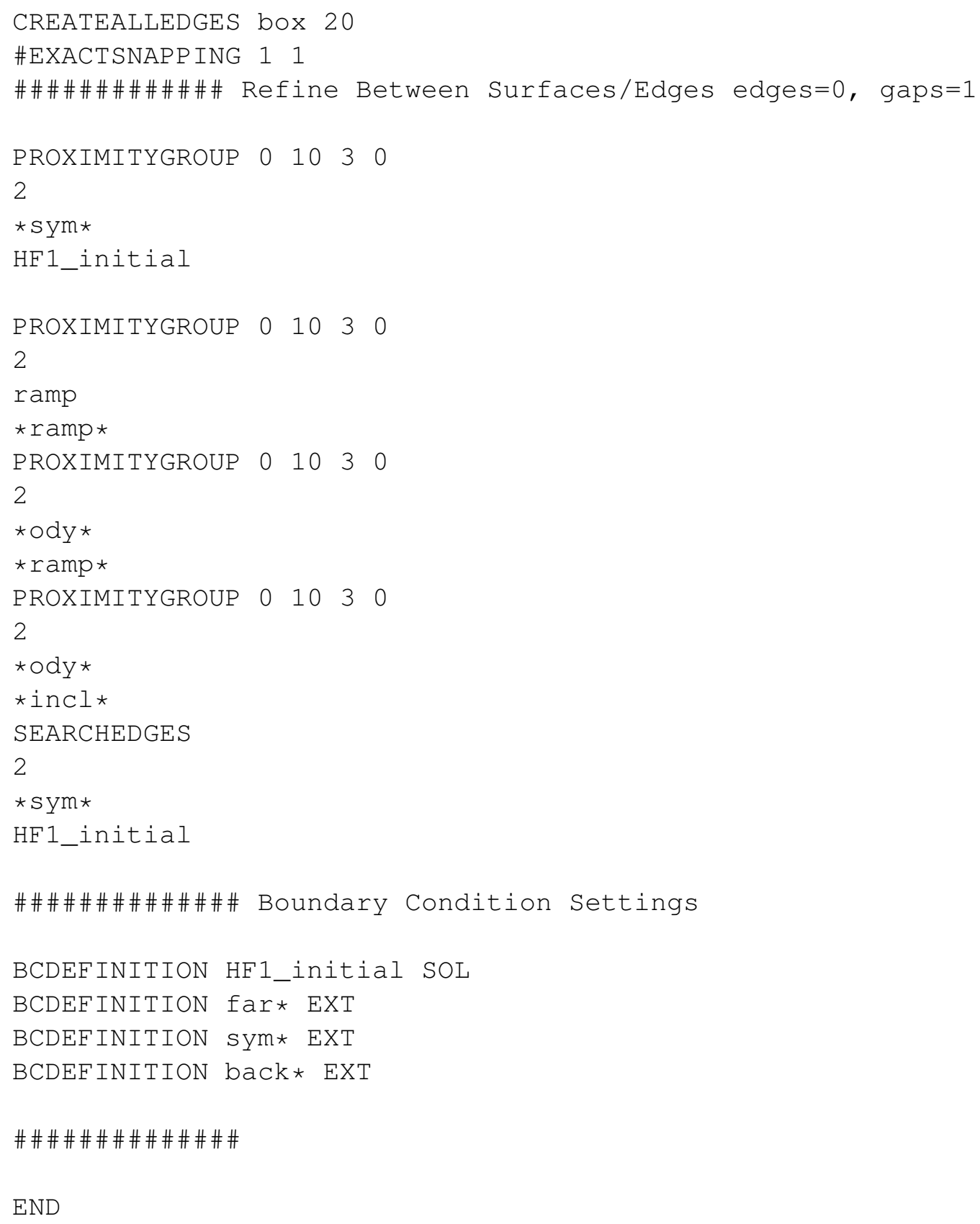




\section{APPENDIX C}

\section{Fun3d Input Script for HIFiRE-1 Fine Mesh}

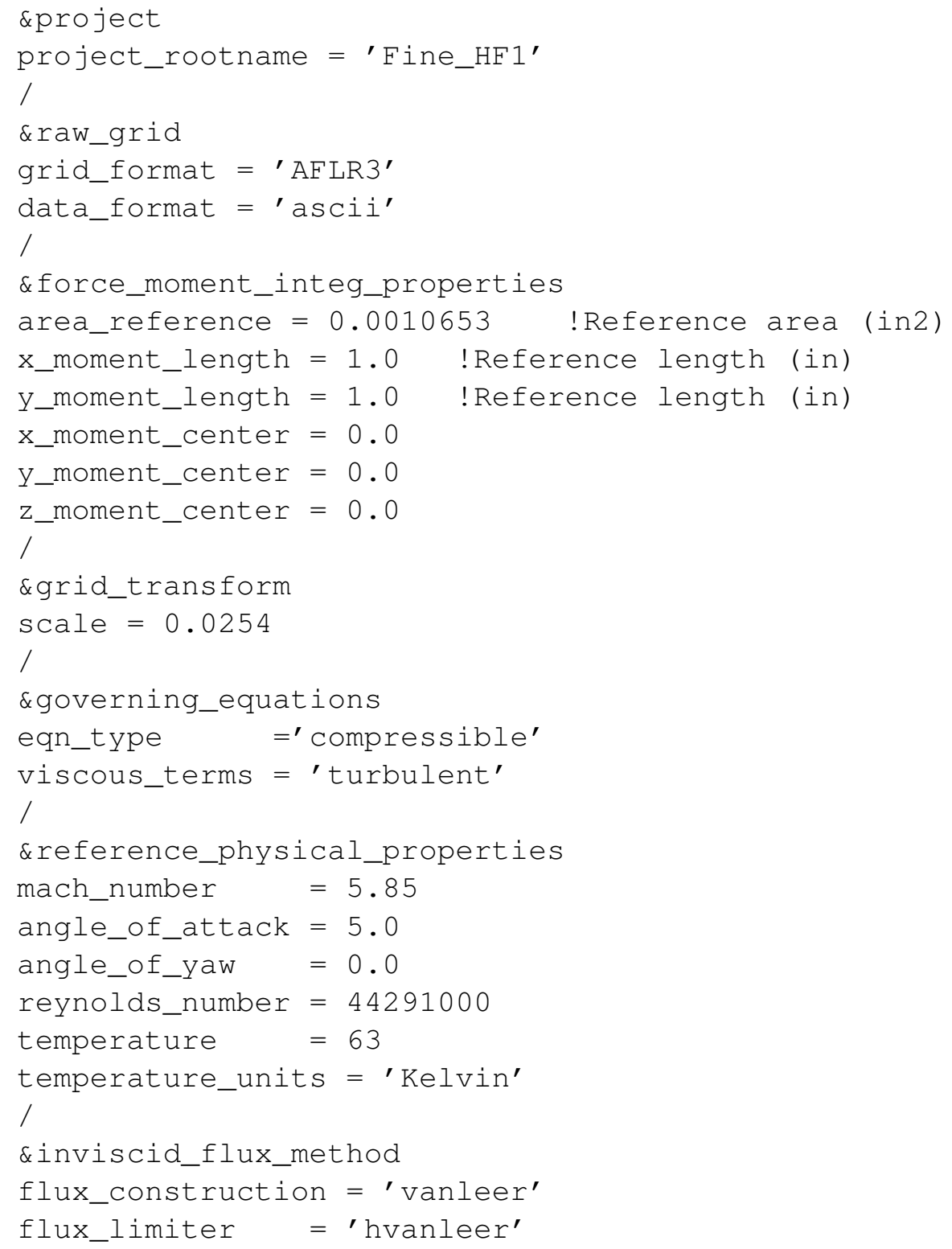




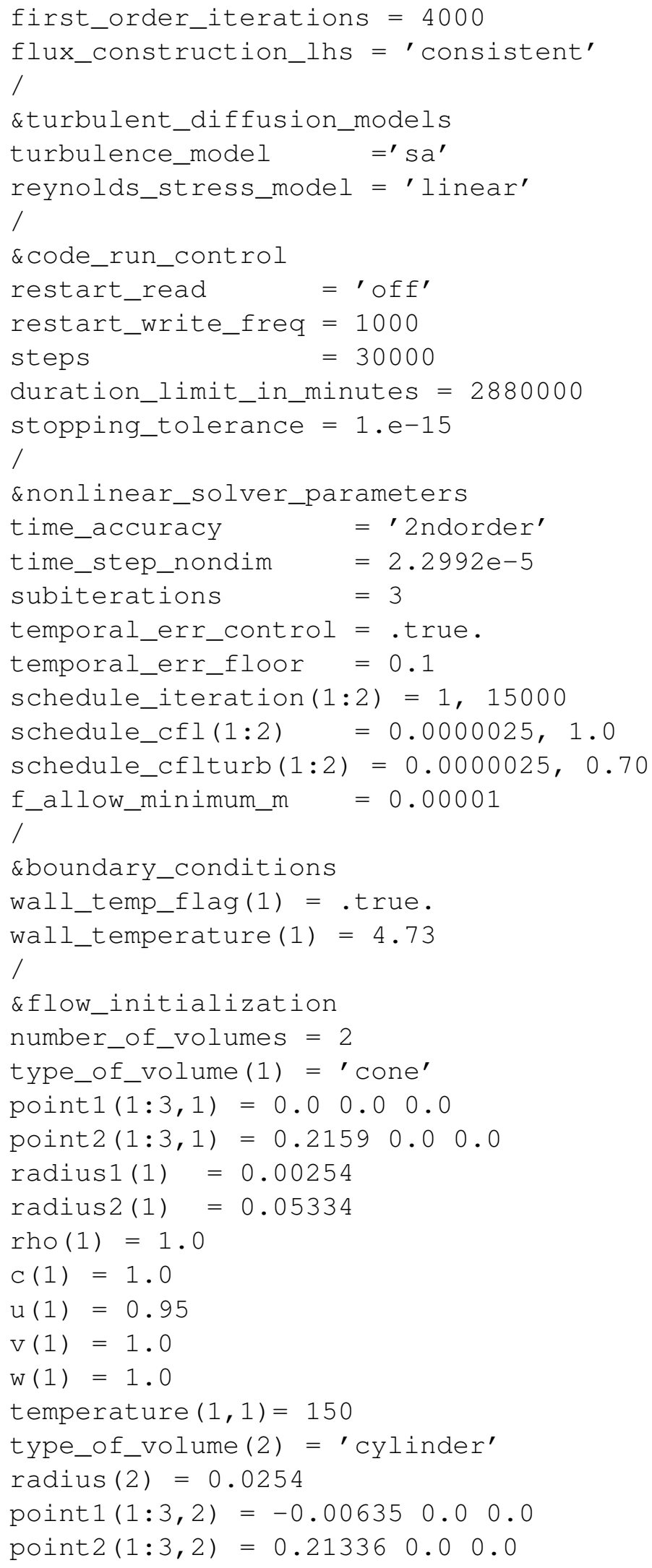




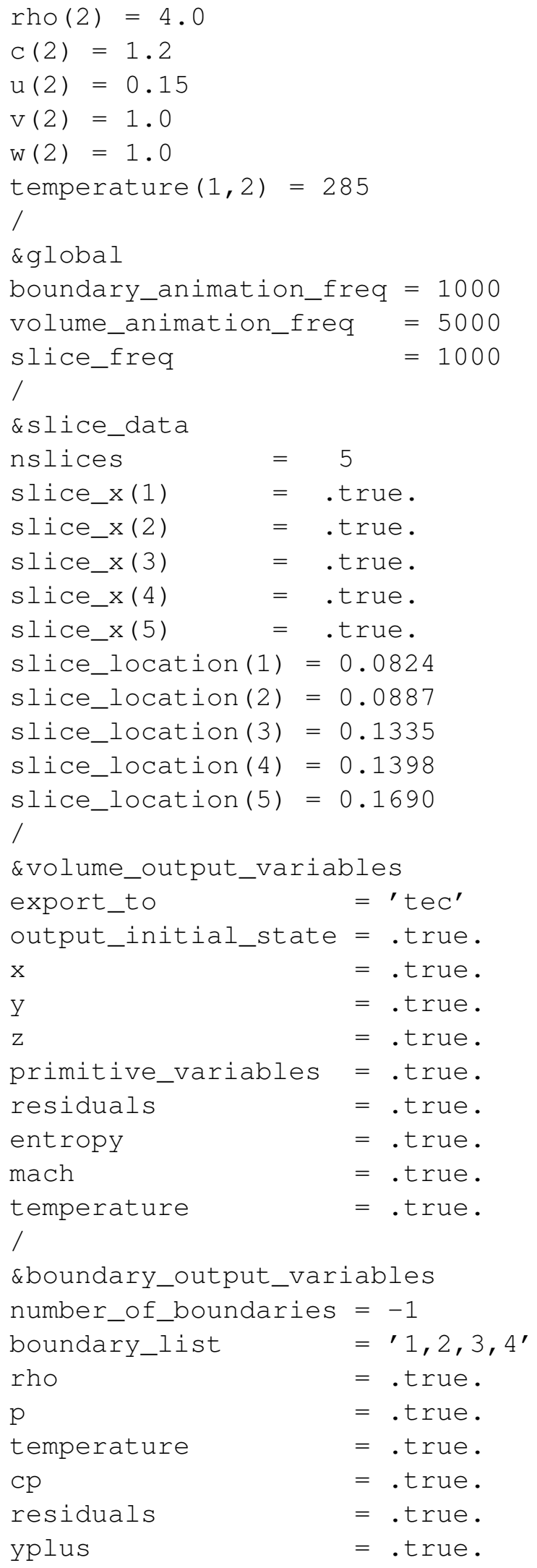


$\mathrm{cq}$

skinfr

heating

blayer

/ $=$.true.

$=$.true.

$=$.true.

$=$.true. 